\title{
Exploring Work-Related Spinal Cord Injuries in Gauteng Province, South Africa
}

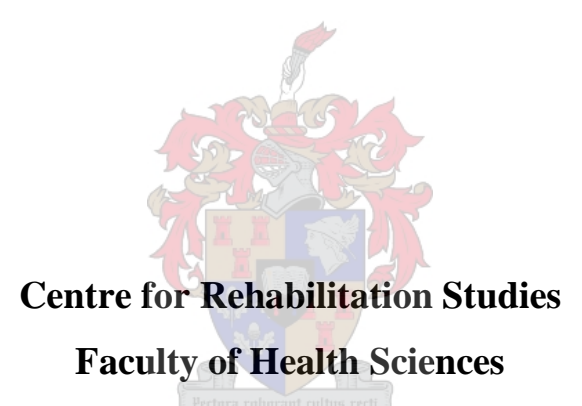

Ntsikelelo Pefile

Research assignment submitted in partial fulfilment of the requirements of the degree of Master of Medical Science Majoring in Rehabilitation

at the University of Stellenbosch

Supervisors: Ms Surona Visagie

Ms Siphokazi Gcaza

O DUFKRा 


\section{DECLARATION}

By submitting this thesis/dissertation electronically, I, Ntsikelelo Pefile, declare that the entirety of the work contained therein is my own, original work, that I am the sole author thereof (save to the extent explicitly otherwise stated), that reproduction and publication thereof by Stellenbosch University will not infringe any third party rights and that I have not previously in its entirety or in part submitted it for obtaining any qualification.

\section{Name: Ntsikelelo Pefile}

\section{Signed:}

Date: 0 DUFKपा

Copyright @ 92010 University of Stellenbosch

All rights reserved 


\section{DEDICATION}

In loving memory of my late parents

Mrs Hombakazi Euphimia Pefile and Mr Abel Nkwenkwe Pefile 


\section{ABSTRACT}

\section{Introduction}

Spinal cord injury ( $\mathrm{SCI}$ ) is devastating and impacts negatively on an individual's functioning and social participation. A good legal framework in South Africa promotes health and safety in the workplace, but there are no specific guidelines regarding prevention of spinal cord injuries. Before any preventative programme can be embarked upon, stakeholders must understand the current situation. Therefore the aim of the study was to describe work-related spinal cord injuries in Gauteng Province, South Africa, to provide employers and policy makers with the information necessary to develop SCI preventative strategies in the workplace.

\section{Methodology}

This was a descriptive, retrospective study in which both quantitative and qualitative methods were utilised. The research was completed in two private rehabilitation units in Gauteng Province, South Africa. During the first (quantitative) phase 135 medical files/records of persons admitted between 1999-2009, who had suffered work-related SCIs, were perused. No sampling was done. Data was collected using a data-collection tool developed by the researcher, the International Classification of Function (ICF) checklist and the Functional Independence Measure+Functional Assessment Measure (FIM+FAM). In the second (qualitative) phase, key informants representing employees who sustained SCI at work were interviewed. Participants were sampled purposively. Data in phase one was analysed using the Statistical Package for the Social Sciences, version 18, and content analysis was used to analyse qualitative data.

\section{Results}

The most affected age range was 26-35. The male-to-female ratio was 6.69:1. Blacks (79.3\%) were more affected by work-related SCIs. The majority of these occurred in urban (49.6\%) and peri-urban (39.3\%) areas. The service (34.8\%), engineering (14.8\%), mining (14\%) and construction (9.6\%) were the most affected industries. The most affected occupations were labourers (25.9\%) and professionals (23.7\%). The security cluster (police and security officers) within the service industry was the most affected (55.3\%). Motor vehicle accidents (48.9\%), violence (20.7\%) and falls (16.3\%) were the major causes of work-related SCIs. The most affected region of the spine was cervical $(41.5 \%)$ although the majority were classified as paraplegic (58.5\% sustained thoracic and lumbar lesions). The majority (51\% of work-related SCIs were classified as complete). The average length of stay (LOS) in-patient rehabilitation was 65.76 days. There was a marginal correlation between LOS and functional FIM+FAM scores ( $\mathrm{r}=-0.32)$. The average cost of rehabilitation was R101 386.84 per person. There were statistically significant functional gains following rehabilitation $(\mathrm{p}=0.000)$. Reemployment levels were high, at 54\%. Participants with lumbar lesions showed statistically higher re- 
employment levels than those with other level lesions $(\mathrm{p}=0.036)$. The findings of phase two of the study showed a degree of non-compliance of employees and employers to safety standards in the workplace.

\section{Conclusions and recommendations}

Findings indicated high levels of motor vehicle accidents and gunshot wounds. Motor vehicle accidents could, in some cases be related to un-roadworthy vehicles or transgressing traffic laws. Employers and employees should adhere to national laws as well as internal health and safety regulations in order to reduce SCI at work. In addition a national database on work-related SCI should be kept. Training of employees and employers in compliance in health and safety matters in the workplace is necessary. Monitoring of compliance by both employees and employers should be vigorous.

Keywords: Work-related, spinal cord injury, Gauteng 


\section{OPSOMMING}

\section{Inleiding}

Individue se funksie en sosiale deelname word negatief beinvloed deur ` $n$ spinaalkoordbesering. Suid Afrika beskik oor `n goeie wetlike raamwerk wat gesondheid en veiligheid in die werkplek bevorder, maar daar bestaan geen spesifieke riglyne vir die voorkoming van spinaalkoordbeserings in die werkplek nie. Dit is belangrik dat alle belanghebbendes die huidge situasie verstaan voordat enige voorkomende programme van stapel gestuur kan word. Die doel van die studie was om werkverwante spinaalkoordbeserings in Gauteng Provinsie, Suid-Afrika, te beskryf, om sodoende werkgewers en beleidmakers te voorsien met die nodige inligting om voorkomende strategieë te ontwikkel.

\section{Metodologie}

Gemengde metodes van data insameling en ontleding is in hierdie beskrywende, retrospektiewe studie gebruik. Die navorsing is in twee privaat Rehabilitasie Eenhede in Gauteng Provinsie, Suid-Afrika gedoen. Gedurende die kwantitatiewe fase van die studie is inligting ingesamel uit 135 mediese rekords van persone wat werkverwante spinaalkoordbeserings opgedoen het en tussen 1999 en 2009 rehabilitasie in een van die twee eenhede ontvang het. Geen steekproefneming is gedoen. Data is ingesamel deur gebruik te maak van 'n data-insameling instrument wat deur die navorser ontwikkel is, die ICF checklist en die FIM+FAM. In die tweede (kwalitatiewe) fase is onderhoude met sleutel informante i.e. werknemers wat spinaalkoordbeserings by die werk opgedoen het, gedoen. Data in fase een is ontleed met behulp van SPSS weergawe 18 en tematiese analise is gebruik om kwalitatiewe data te analiseer.

\section{Resultate}

Die mees geaffekteerde ouderdomsgroep was 26-35. Die verhouding tussen mans en vrouens wat beserings opgedoen het was 6.69:1. Lede van die swart bevolkingsgroep het die mees algemeenste voorgekom onder die deelnemers $(79,3 \%)$. Die meerderheid van beserings het in stedelike $(49,6 \%)$ en semi-stedelike gebiede $(39,3 \%)$ voorgekom. Die industrieë waar beserings die mees algemeenste voorgekom het was dienslewering (34,8\%), ingenieurswese (14,8\%), mynbou (14\%) en konstruksie (9.6\%). Arbeiders $(25,9 \%)$ en professionele mense $(23,7 \%)$ was die beroepe waarin dit die meeste voorgekom het. Polisie en sekuriteits beamptes (55.3\%) was die meeste geraak in die diens industrie. Motorvoertuigongelukke (48,9\%), geweld $(20,7 \%)$ en valle $(16,3 \%)$ was die grootste oorsake van die beserings. Die meeste beserings het aan die servikale (41.5\%) deel van die rug voorgekom, alhoewel daar in totaal meer persone was met paraplegie (58.5\% torakale en lumbale letsels). Die meeste beserings was volledig (51\%). Die gemiddelde lengte van binne pasient verblyf was 65,76 dae. Daar was ' $\mathrm{n}$ marginale korrelasie tussen lengte van verblyf en Funksionele FIM+FAM tellings $(r=-0.32)$.

Die gemiddelde koste van rehabilitasie was R101 386.84 per persoon. Deelnemers het statisties 
beduinde funksionele vordering gemaak tydens rehabilitasie $(\mathrm{p}=0.000)$. Vier en vyftig persent van deelnemers het teruggekeer na `n werk na rehabilitasie. `n Lumbale letsel het deelnemers se kanse om terug te keer na werk statisties beduidend verhoog $(\mathrm{p}=0.036)$. Bevindinge in die tweede fase van die studie het daarop gedui dat werknemers en werkgewers nie altyd veiligheidsreëls en regulasies in die werkplek gevolg nie het nie.

\section{Gevolgtrekkings en aanbevelings}

Motorvoertuigongelukke en skietwonde het tot baie van die beserings gelei. In sommige gevalle kon die motorvoertuigongeluk toegeskryf word aan onpadvaardige voertuie of oortreding van verkeerswette. Werkgewers en werknemers moet aan landswette sowel as interne veiligheids reëls gehoorsaam wees om die voorkoms van spinaalkoordbesering in die werkplek te verminder. Voorts moet ' $\mathrm{n}$ nasionale databasis van werk verwante spinaalkoordbeserings in die lewe geroep word. Werknemers en werkgewers moet opgelei word ten opsigte van veiligheid in die werkplek. Die nakoming van veiligheidsreels en regulasies deur beide werknemers and werkgewers moet ferm gemonitor word.

Sleutelwoorde: Werk verwante, spinaalkoordbesering, Gauteng 


\section{ACKNOWLEDGEMENTS}

I am indebted to my supervisors Ms Surona Visagie and Ms Siphokazi Gcaza, under whose guidance and constant encouragement this research assignment was completed.

I wish to express my sincere gratitude to Ms Nina Strydom: without her invaluable support this study would not have started at the research sites. And my thanks to the staff at all research sites: Life Entabeni (where the pilot study was conducted), Life New Kensington Clinic and Life Riverfelt Lodge.

I would like to thank Ms Fikile Nkwanyana and Prof. Nel for their assistance in the statistical analysis of the data, as well as Melanie Stark for editing the final manuscript.

I am grateful to Mr. Lucky Khotha for his invaluable assistance during the data collection phase of the study, and to Ms Lindisa Xundu for her much appreciated support through this process.

I am also thankful to my colleagues at the University of KwaZulu-Natal, Physiotherapy Division, for their support during my sabbatical leave, not forgetting my bosses (Dr. S.S. Maharaj, and Professors J. Van Heerden and S. Essacks) for granting me the time off to complete the study. I am truly grateful.

To my sister, Mrs Verusia Chetty, I know she will always stand behind me. 


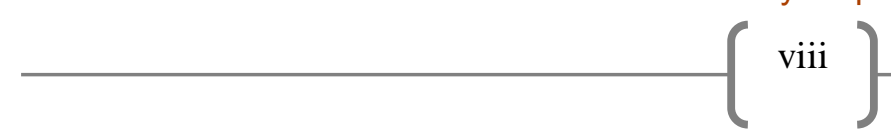

\section{OPERATIONAL DEFINITIONS}

Descriptive epidemiology: Provides a description of the occurrence of disease and other healthrelated characteristics in human populations in terms of person, place and time (Joubert, Ehrlich, Katzenellenbogen and Abdool Karim, 2007).

Epidemiology: Epidemiology studies the distribution and determinants of conditions and events related to health-related characteristics in populations (Joubert et al., 2007).

Extent of spinal cord injury: Refers to the extent of neurological damage, which is either complete or incomplete. If partial preservation of sensory and or motor function is found below the neurological level and includes the lowest sacral segment, the injury is defined as incomplete. The term complete injury is used when there is an absence of sensory and motor function in the lowest sacral segment of the spine (O’ Connor, 2001).

Incidence: Is the number of new cases of a disease over a specific period of time (Joubert et al., 2007).

Neurological level: The most caudal level of the spine that exhibits intact sensory and motor functioning bilaterally (Somers, 2001).

Paraplegia: Loss of motor and/or sensory function of the trunk and lower limbs secondary to damage of the neural elements within the spinal canal at the thoracic, lumbar or sacral segments of the spinal cord (Bromley, 2006).

Quadriplegia: Loss of motor and or sensory function in the trunk and all four limbs secondary to damage of the neural elements within the spinal canal of the cervical segment of the spinal cord (Bromley, 2006).

Spinal cord injury: A spinal cord injury is an insult to the spinal cord resulting in a temporary or permanent loss, of its normal motor, sensory or autonomic functions (Somers, 2001).

Work-related spinal cord injuries: These are injuries to the spinal cord that occur in the workplace and/or where the activity of the person at the time of injury was specified as working for income. 


\section{TABLE OF CONTENTS}

DECLARATION.........................................................................................................................................................

DEDICATION..................................................................................................................................................ii

ABSTRACT ....................................................................................................................................................ii

OPSOMMING................................................................................................................................................. v

ACKNOWLEDGEMENTS ...............................................................................................................vii

OPERATIONAL DEFINITIONS .............................................................................................................viii

TABLE OF CONTENTS ...................................................................................................................... ix

LIST OF TABLES AND FIGURES...........................................................................................................

LIST OF ACRONYMS .......................................................................................................................

CHAPTER 1: INTRODUCTION......................................................................................................1

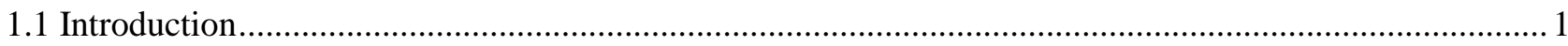

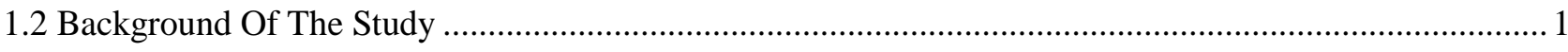

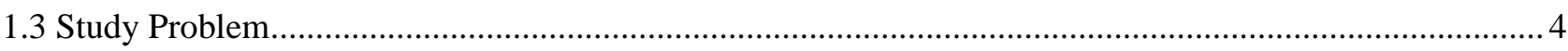

1.4 Aims Of The Study ………………………………………………………………………...

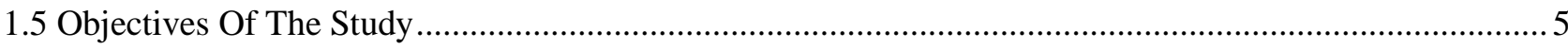

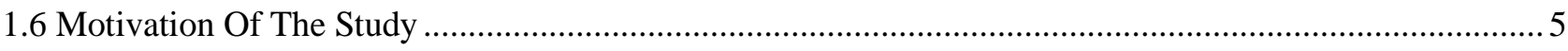

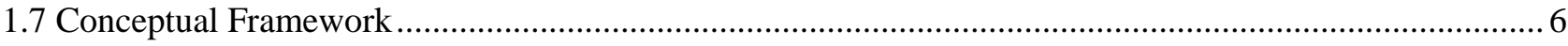

1.8 Significance Of The Study …………………………………………………………………...

1.9 Outline Of The Study ................................................................................................................

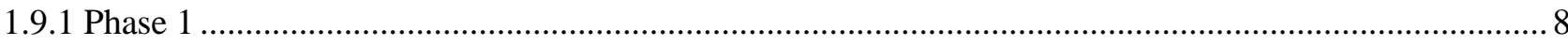

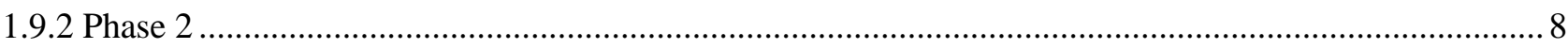

1.10 Chapter Summary …………………………………………………………………………9

CHAPTER 2: LITERATURE REVIEW ..............................................................................................10

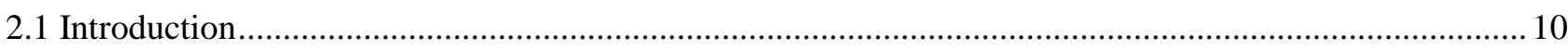

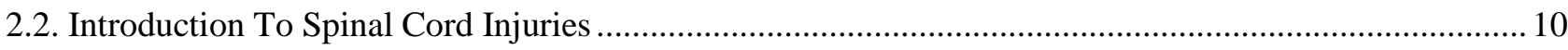

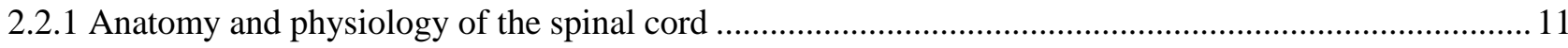

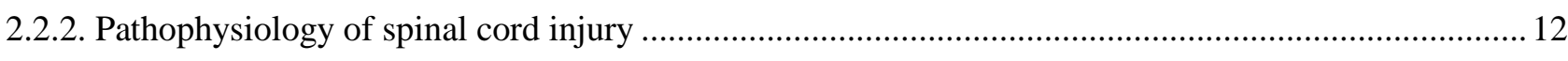

2.2.3 Classification of spinal cord injuries........................................................................................... 14 
2.2.4 Rehabilitation of spinal cord injuries 15

2.2.5 Community integration of people with spinal cord injuries............................................................ 17

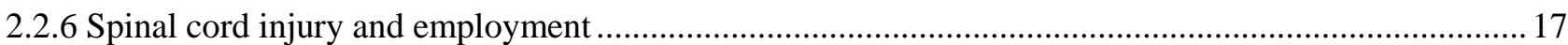

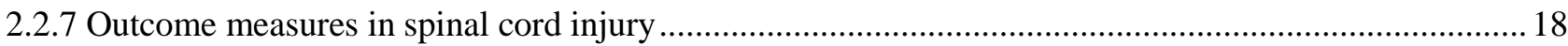

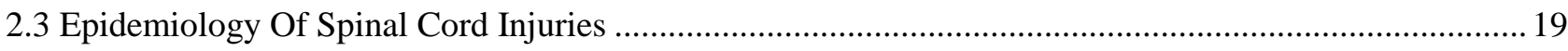

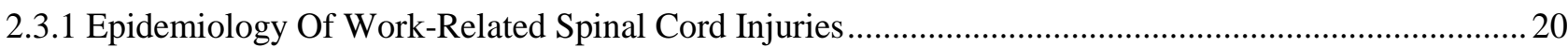

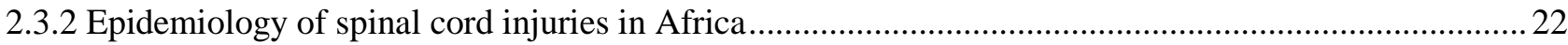

2.3.3 Epidemiology of spinal cord injuries in developed countries ............................................................29

2.3.4 Length of stay, mortality rate and complications of spinal cord injuries ............................................33

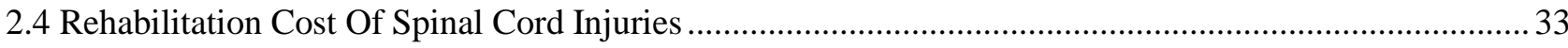

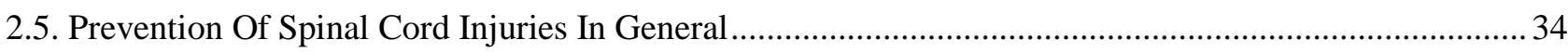

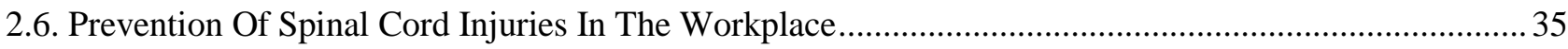

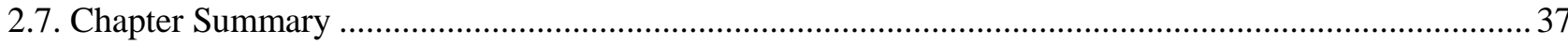

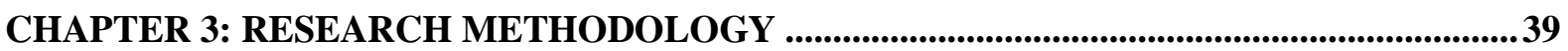

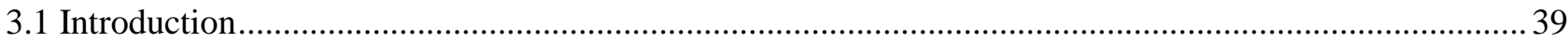

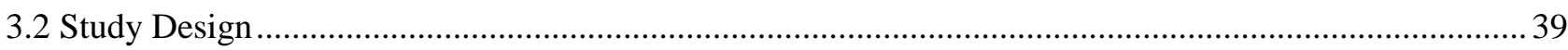

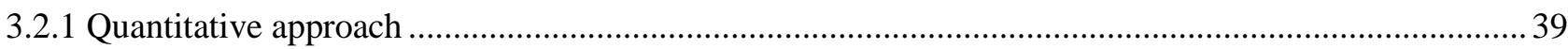

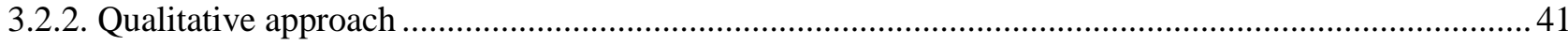

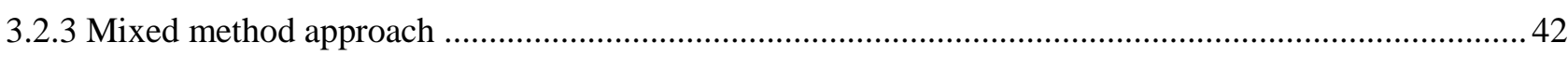

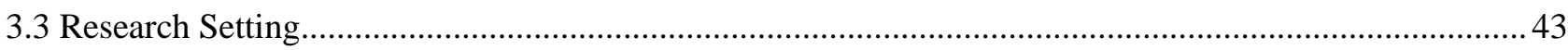

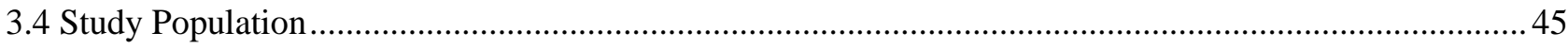

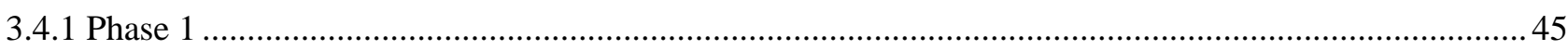

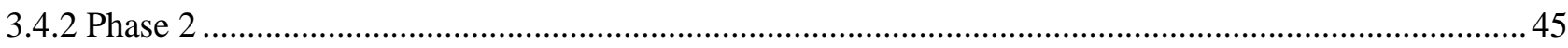

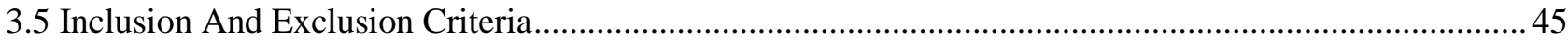

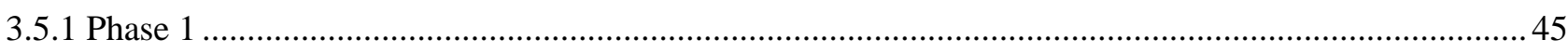

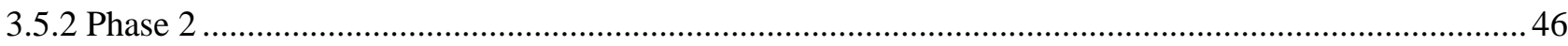

3.6 Sampling Methods And Sample Size .............................................................................................

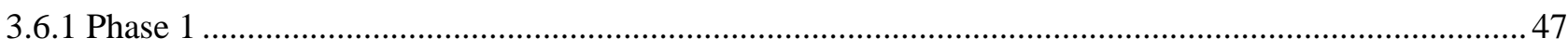

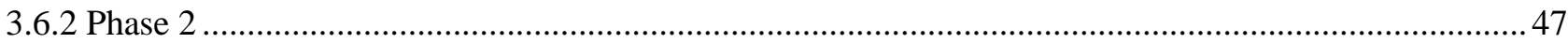


3.7 Data Collection Tools .48

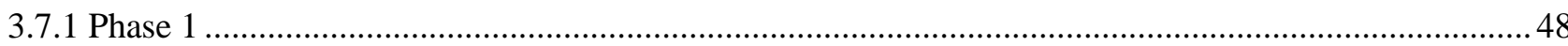

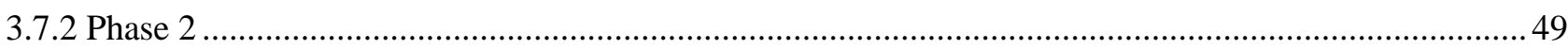

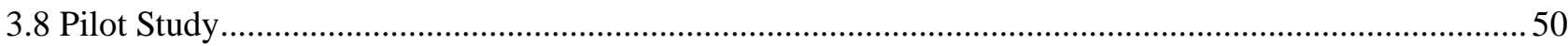

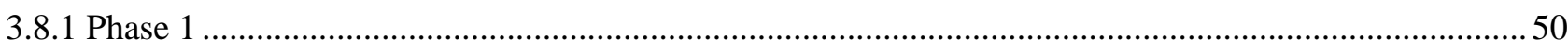

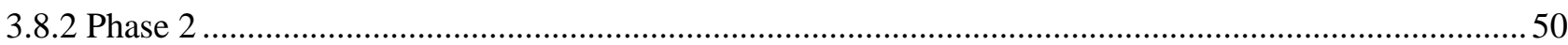

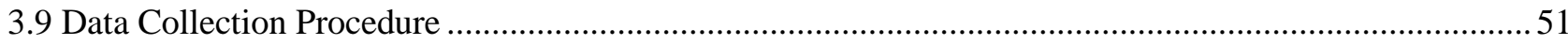

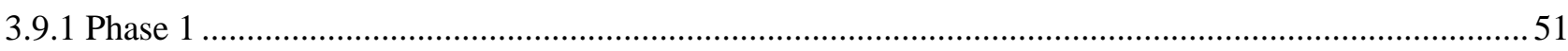

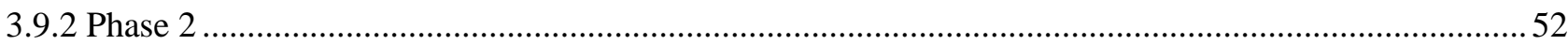

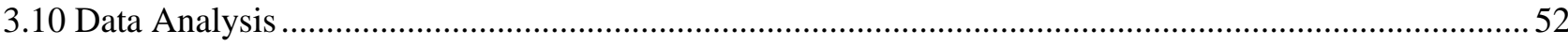

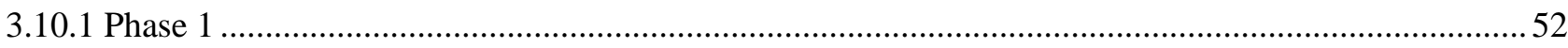

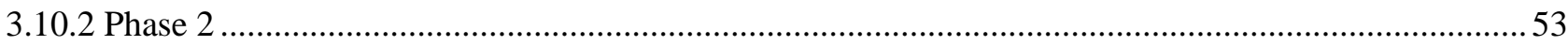

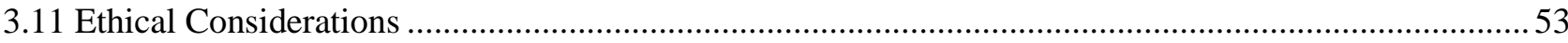

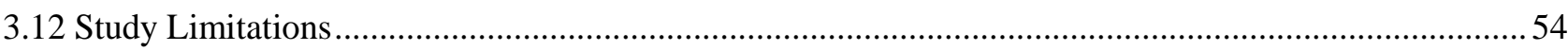

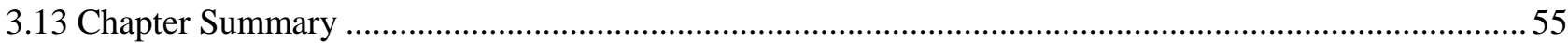

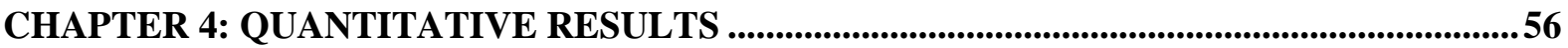

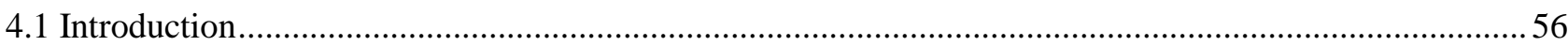

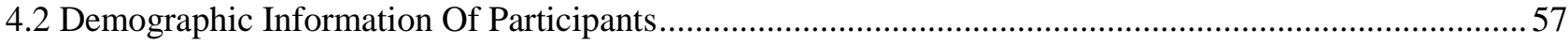

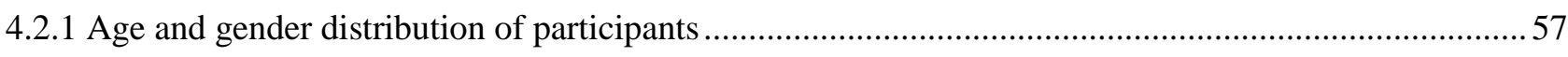

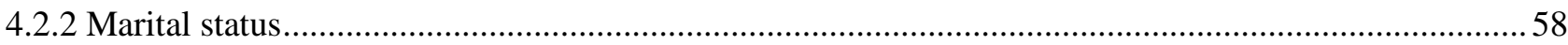

4.2.3. Population group and geographical distribution of places of employment........................................59

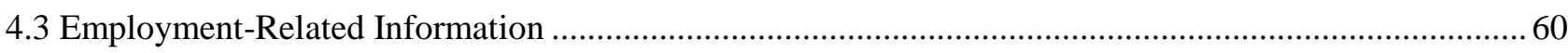

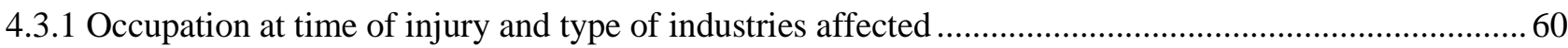

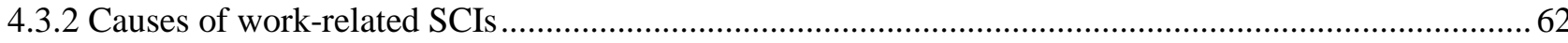

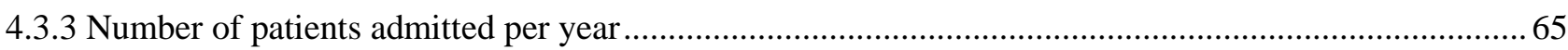

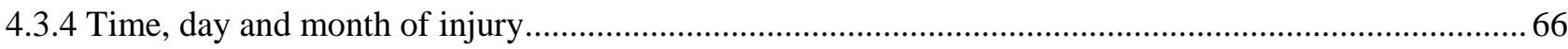

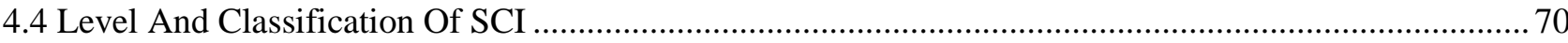

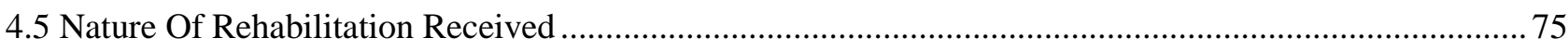

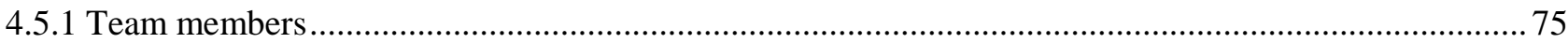


4.5.2 Approaches to rehabilitation provided 75

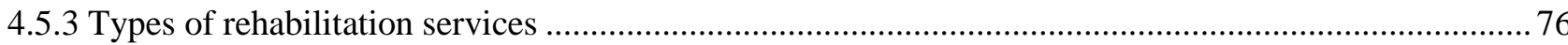

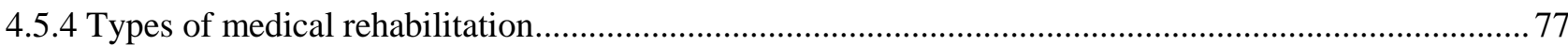

4.5.5 Assistive devices issued during rehabilitation ................................................................................. 78

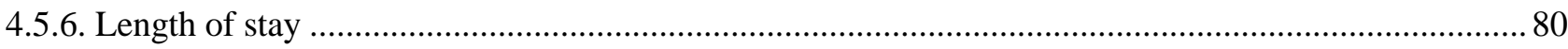

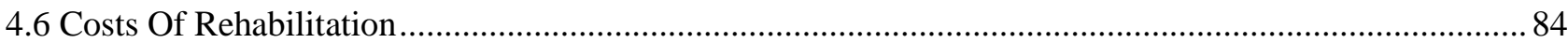

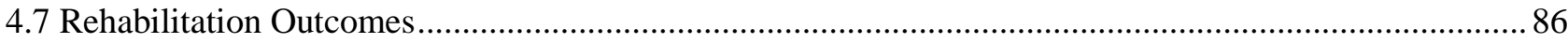

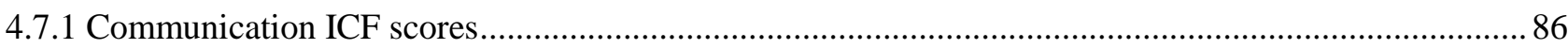

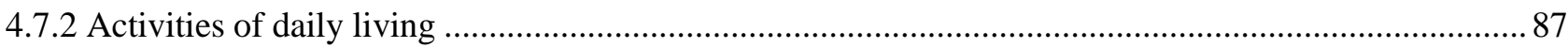

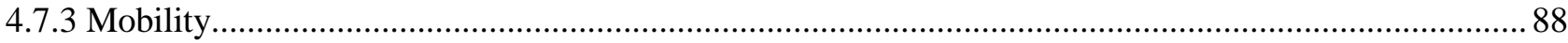

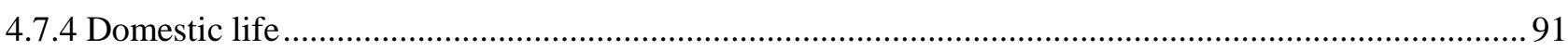

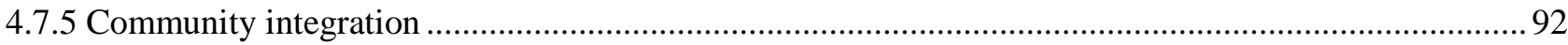

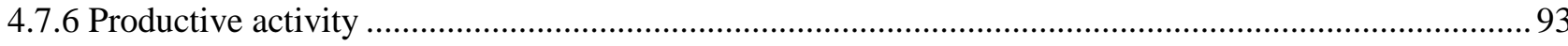

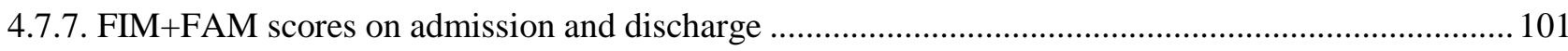

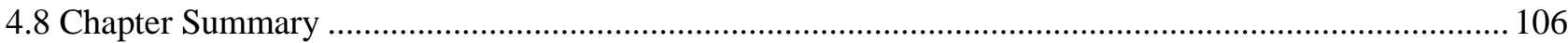

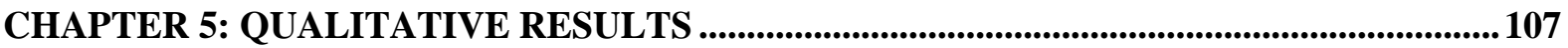

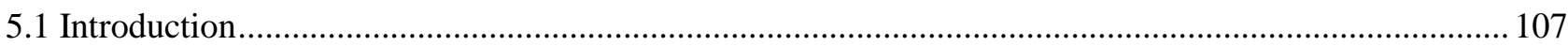

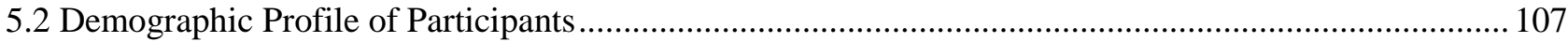

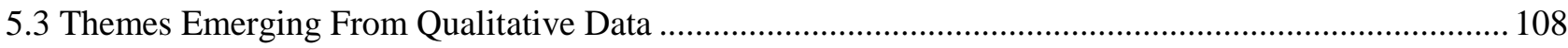

5.3.1. Poor compliance to health and safety regulations in the workplace …….......................................... 108

5.3.2 Management of an injured employee in the workplace .................................................................... 111

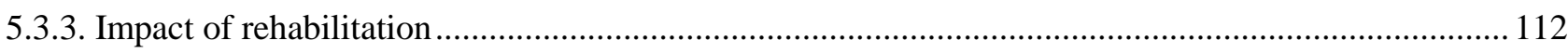

5.3.4 Recommendations to improve health and safety in the workplace ................................................. 112

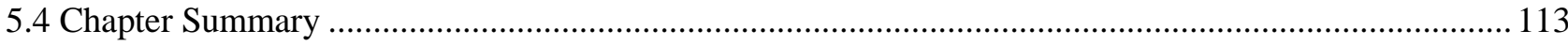

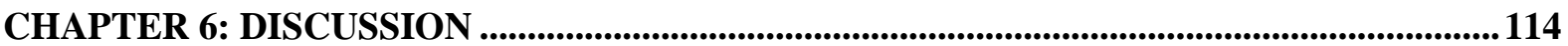

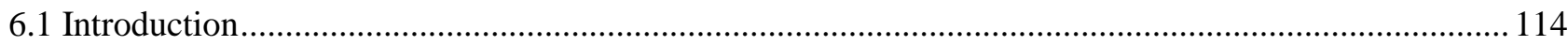

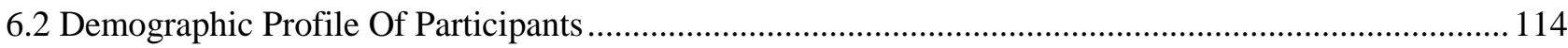

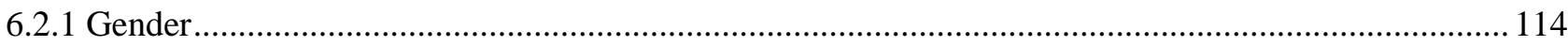

6.2.2 Age 
6.2.3 Marital Status

6.2.4 Population Group

6.3 Occupations And Industries Affected.

6.4 Causes Of Work-Related Spinal Cord Injuries

6.5 Occurrence Of Spinal Cord Injuries In The Study Setting

6.6 Level and Classification of Spinal Cord Injury

6.7 Rehabilitation Provided To The Study Population

6.8 Duration Of In-Patient Rehabilitation.

6.9 Costs Of Rehabilitation

6.10 Outcomes Of Rehabilitation

6.11 Employment Status On Discharge

6.13 Chapter Summary

CHAPTER 7: CONCLUSION AND RECOMMENDATIONS

7.1 Conclusion

7.2 Recommendations

7.2.1 Preventative Framework

7.2.2 Recommendations To Employers (In relation to Section 8 (1 and 2) of the OHSA, 1993).....

7.2.3. Recommendations To Employees

7.2.4 Recommendations To Rehabilitation Facilities

7.2.5 Recommendations To Individuals With Work-Related SCI And Their Families......

7.2.6 Recommendations To Government, Policy Makers And Business

REFERENCES

1.1 Appendix A: Data Collection Tool: Information from the Life Rehabilitation Centre

1.2 Appendix B: Interview Schedule: Employer 165

1.3 Appendix C: Interview Schedule: Employee

1.4 Appendix D: Interview Schedule for persons who sustained Spinal Cord Injuries

1.5 Appendix E: Participant Information Leaflet And Consent Form. 
1.6 Appendix F: Application for Access to Claimant's Files

182

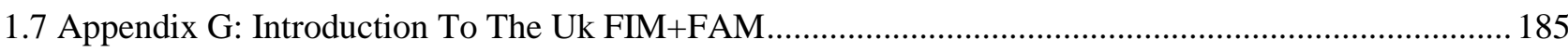




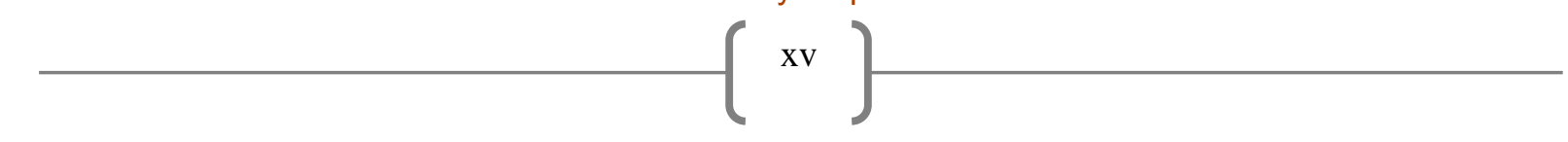

\section{LIST OF TABLES AND FIGURES}

Figure1.1 Study Conceptual Framework

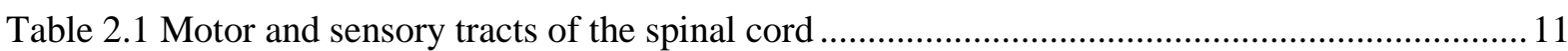

Table 2.2 Scales used to classify SCI according to completeness of the injury .................................. 15

Table 2.3: Rehabilitation team members and their roles in SCI rehabilitation (adapted from Saulino,

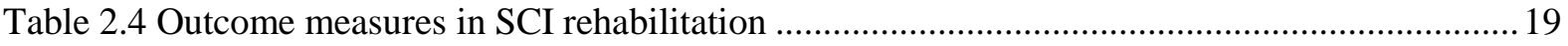

Table 2.5 Methodology and demographic findings from studies on work-related SCIs .....................20

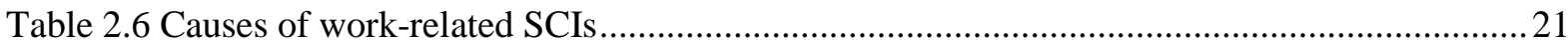

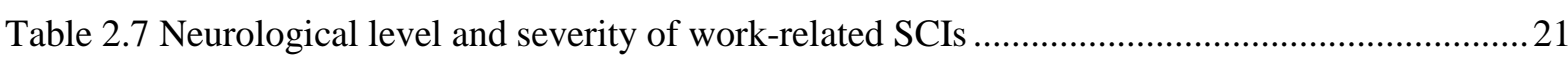

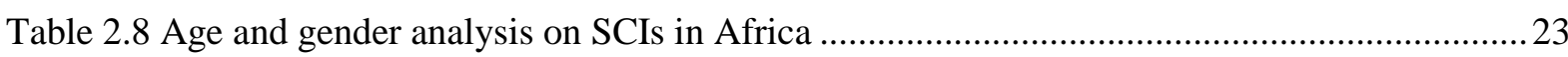

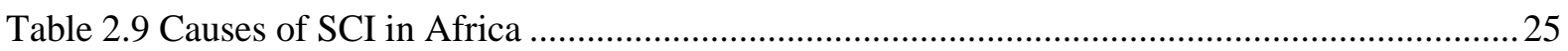

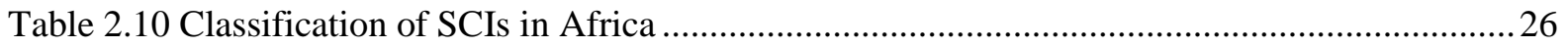

Table 2.11 Length of stay, mortality rate and complications of SCIs in Africa ..................................27

Table 2.12 Age and gender analysis on SCIs in developed countries................................................. 30

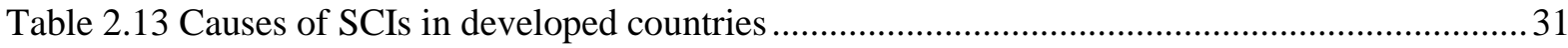

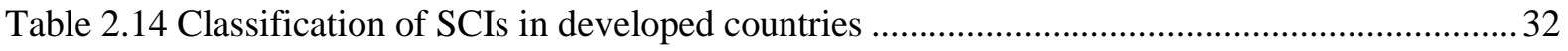

Figure 3.1 Epidemiological triangle (Adapted from Joubert et al., 2007) ............................................. 40

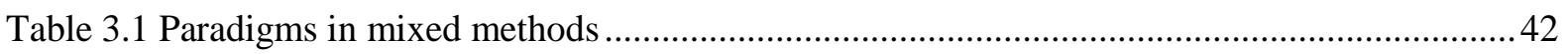

Table 3.2 Population estimates of Gauteng Province from 1996 to 2007 (Stats SA, 2007) ................ 43

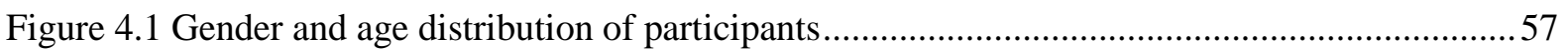

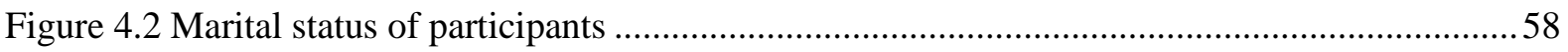

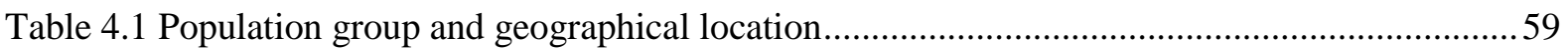

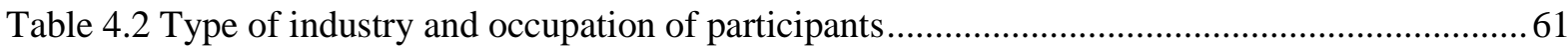

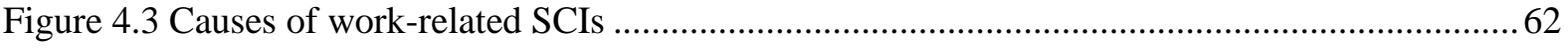

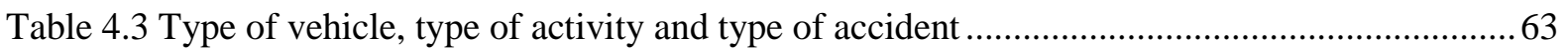

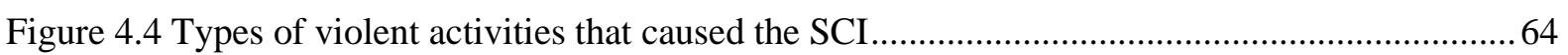

Figure 4.5 Number of patients admitted per year during study period ............................................. 65

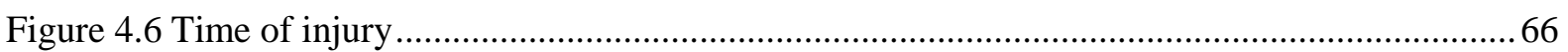

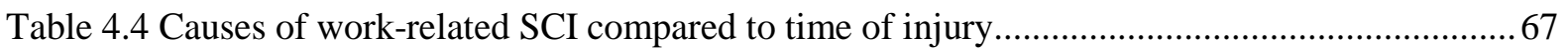

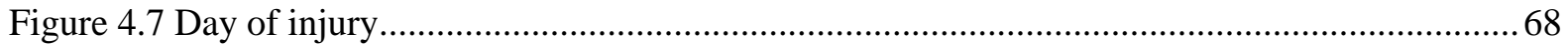

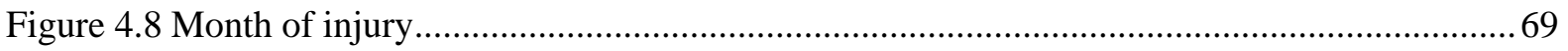

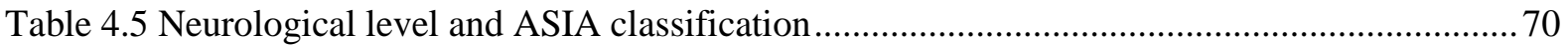

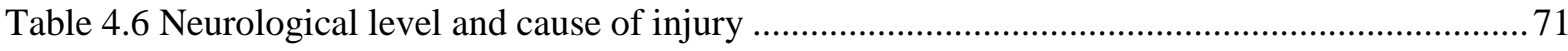

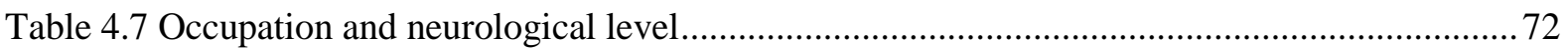




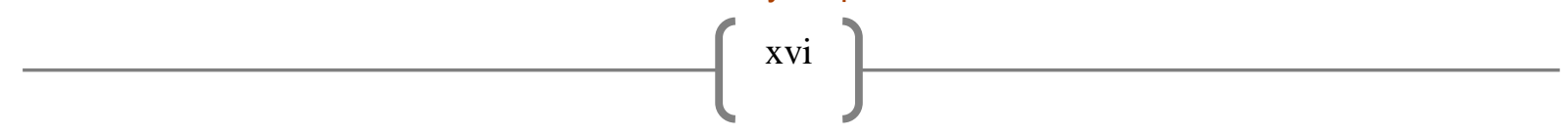

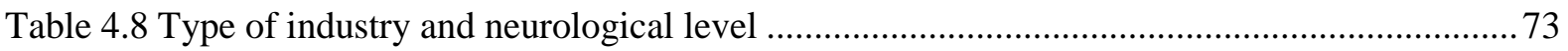

Table 4.8 Type of industry and neurological level (continued) ……................................................... 74

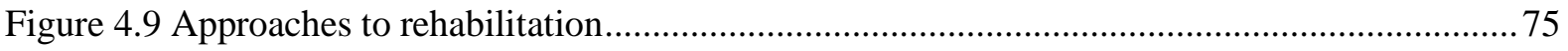

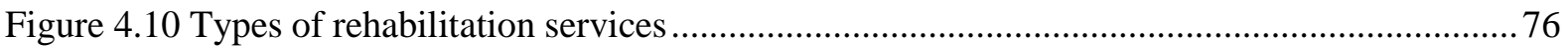

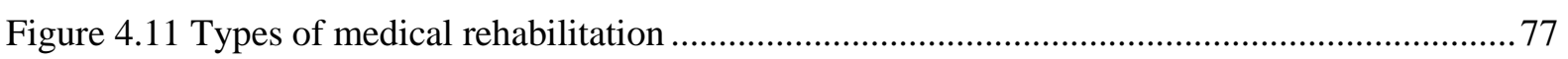

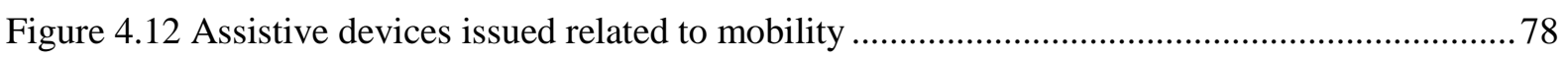

Figure 4.13 Assistive devices related to activities of daily living....................................................... 79

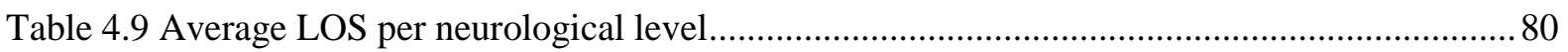

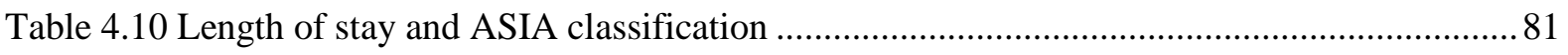

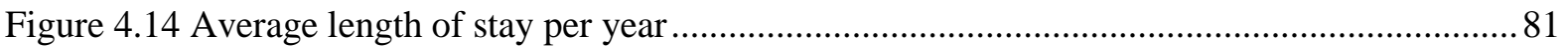

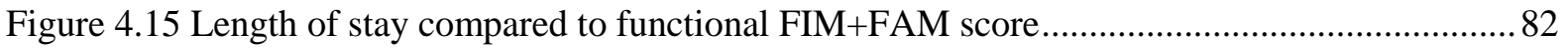

Figure 4.16 Length of stay compared to cognitive FIM+FAM score ................................................. 82

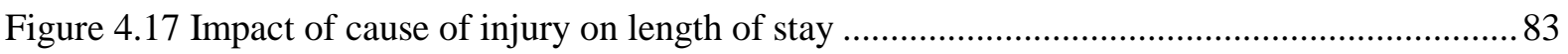

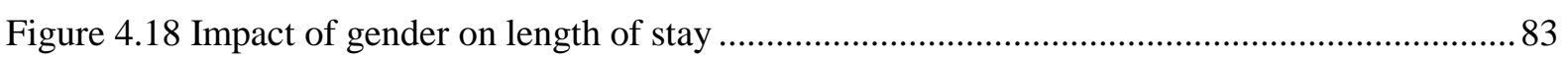

Table 4.11 Estimated costs per neurological level (ZAR) ............................................................. 84

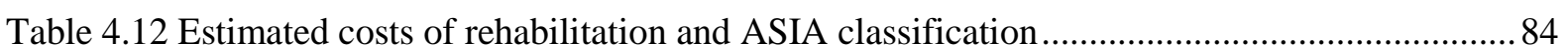

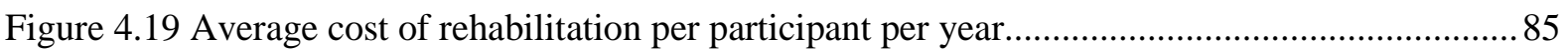

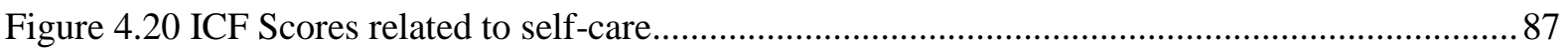

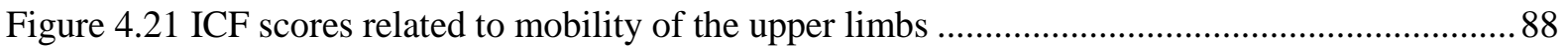

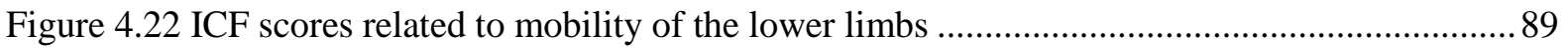

Figure 4.23 ICF scores related to mobility around the house ............................................................ 90

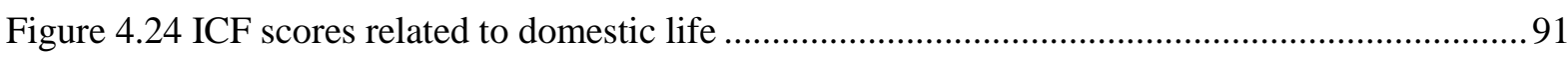

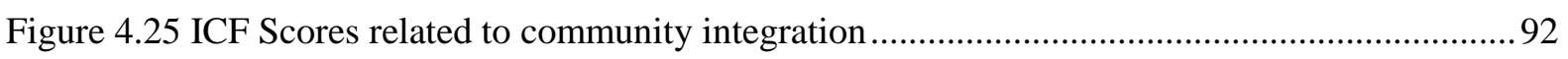

Figure 4.26 ICF Scores related to productive activity .................................................................... 93

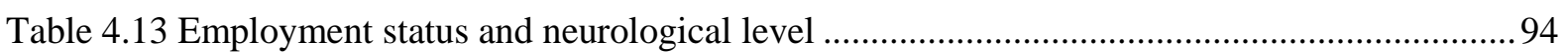

Table 4.14 Employment status at discharge and classification of injury ............................................95

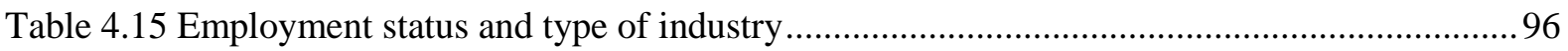

Table 4.15 Employment status and type of industry (continued) ......................................................97

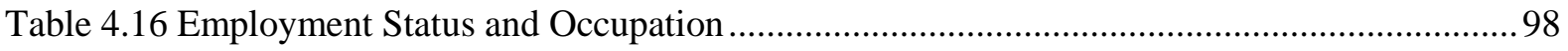

Table 4.17 Employment status (ES) and population group (PG) .................................................... 99

Table 4.18 Geographical locations and employment status........................................................... 100

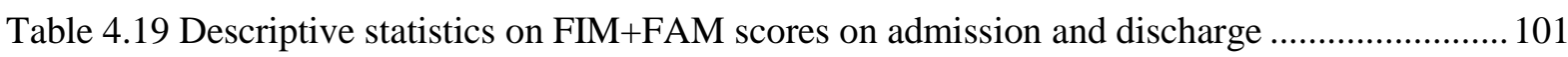

Table 4.20 Descriptive statistics on functional FIM+FAM scores per neurological level (NL)......... 102

Table 4.21 Descriptive statistics on cognitive FIM+FAM scores per neurological level (NL) .......... 103

Table 4.22 Individual differences in FIM+FAM and FAM scores (cervical region of the spine) ....... 104

Table 4.23 Individual difference in FIM+FAM scores (thoracic region of the spine)........................ 104

Table 4.24 Individual differences in FIM+FAM scores (lumbar region of the spine)........................ 105 


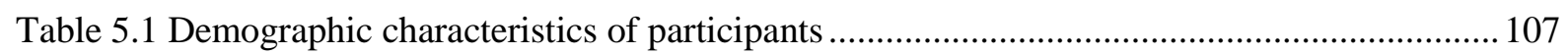

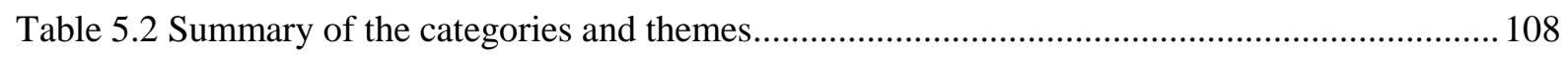

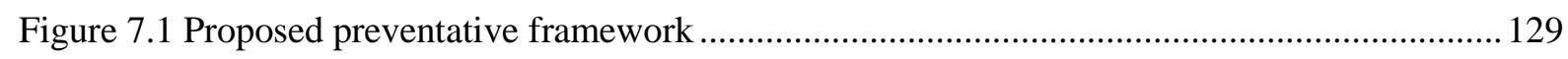




\section{LIST OF ACRONYMS}

ADL

ASIA

COIDA

FAM

FIM

GDP

ICF

LOS

MHSA

MVA

NGO

NL

OHSA

OT

PPE

PT

RDS

SCI

SETA

SHE

UCT

UNESCO

USA

USD

ZAR
Activities of Daily Living

American Spinal Injury Association

(South African) Compensation for Occupational Injuries and Diseases Act

Functional Assessment Measure

Functional Independence Measure

Gross Domestic Product

International Classification of Function, Disability and Health

Length of Stay

(South African) Mine Health and Safety Act, 29 of 1996

Motor Vehicle Accident

Non-governmental organisation

Neurological Level

(South African) Occupational Health and Safety Act, 85 of 1993

Occupational Therapist

Personal Protective Equipment

Physiotherapist

Respiratory Distress Syndrome

Spinal Cord Injury/ies

Skills Education Training Authorities

Safety, health and environment

University of Cape Town

United Nations Educational, Scientific and Cultural Organisation

United States of America

United States dollar/s

South African Rands 


\section{CHAPTER 1: INTRODUCTION}

\subsection{Introduction}

This chapter provides an introduction to the study. It presents the background of the study as well as the problem statement. The motivation, aim and specific objectives of the study are stated. A conceptual framework which provided a foundation for this study is explained. It concludes with the significance of the study and a brief outline of the rest of the study.

\subsection{Background Of The Study}

Spinal cord injury (SCI) is a serious neurological injury with varying degrees of motor and sensory functioning loss and possible sphincter disturbance, which are permanent and cannot be cured or reversed in most cases (Harvey 2008). Severe injury to the spinal cord produces major disruptions to the individual's quality of life, his or her family and to society in general. In most instances, the person with SCI has to abandon his job, and is significantly affected (Ekong and Tator, 1985; O'Connor, 2002a).

Spinal cord injuries occur everywhere throughout the world, with an estimated annual incidence of 15 to 40 cases per million, with causes varying from motor vehicle accidents and interpersonal violence to recreational activities and occupational-related injuries (Sekhon and Fehlings 2001). Ackery, Tator and Krassioukov (2004) found the incidence rate of SCI in different countries to range between 11.5 and 57.8 cases per million of the general population. Authors found it difficult to estimate a worldwide phenomenon, due to inconsistency in data reporting and sources of data (Ackery et al., 2004).

It is estimated that occupational-related SCI accounted for about $13 \%$ of all traumatic cases of SCI in Australia (O' Connor, 2001). The incidence of work-related SCI in the same study was estimated to be 4 cases per million of the population per year from 1986 to 1997 (twelve year period), which translated to a mean of 32 cases of SCI occurring each year in the work environment (389 in total). O'Connor (2001) argues that, while the rate of occurrence of work-related SCIs in the study was low, the severity of the injury has an enormous consequence in the personal life of individuals as well as their economic well-being. Rosenberg, Gerhart and Whiteneck (1993) reported 74 cases of workrelated SCI over a five year period in the United States of America (USA). In a study by Ekong and Tator (1985), 25 cases of work-related SCI were described over a five year period in Canada. 
Internationally, the most commonly cited causes of work-related SCI are falls; motor vehicle accidents (MVA); being struck or crushed by an object, machinery or person; gunshot wounds; stabbing; and skiing accidents (Rosenberg et al., 1993, O'Connor, 2001). The industries most affected were agriculture, construction, mining, manufacturing (Rosenberg et al., 1993, O'Connor, 2001). Webster, Giunti, Young, Pransky and Nesathurai, (2004) added the retail, utility and service industries. These injuries often affect young adults (90\% males) and usually result in irreversible neurological damage and extensive medical and rehabilitation costs (Griffin, Opitz, Kurland, Ebersold and O'Fallon, 1985).

No information on the burden of cost of SCI in South Africa could be found. However, there is extensive data from the United States. The cost per injury can amount to hundreds of thousands of United States dollars (USD) in lost salary and medical and rehabilitation costs (Ekong and Tator, 1985). According to the Centre for Disease Control, the US government spends 9.7 billion dollars on the treatment of SCI each year and in 2003, management of pressure sores alone (a common but preventable secondary complication of SCI) costs approximately one billion dollars. Post-discharge care (home modifications and personal assistance) costs about fifteen thousand USD per year (French, Campbell, Sabharwal, Nelson, Palacios, Gavin-Dreschnack, 2007). In the US, the management of an individual who sustains SCI is very costly, exceeded only by respiratory distress syndrome (RDS) in very young babies, and it also requires the second longest hospital stay (Winslow, Bode, Felton, Chen, Meyer, 2002).

Occupational injuries in general have immense social and economic implications for everyone involved including individuals, their families and their communities, with high direct and indirect costs for society as a whole (Rosenberg et al., 1993). The occupational accidents and diseases total costs are estimated to be between one to three percent of the Gross Domestic Product (GDP) in various countries (Rosenberg et al., 1993). Direct costs include compensation and medical costs, costs associated with damage in the work environment and the costs of interruption of production. Indirect expenses include the costs of source of revenue lost, earnings to dependents and the costs associated with care-giving by families and communities (Hermanus, 2007).

SCI injuries in Africa mostly affect individuals between 15-40 years of age. Males are more affected than females. The most common cited causes of SCI in Africa are road traffic accidents, falls and violence, including gunshots, stabbings and assaults (Iwegbu, 1983; Odendaal, 1991 a and b; Couto and Levy, 1994). In South Africa during the early 1980s road traffic accidents were the major causes of SCI (Odendaal, 1991a and b). This trend changed during the 1990s when violence became the most common cause of SCI (Hart and Williams, 1994; Velhamos, Degiannis, Hart, Souter and Saaidia, 
1995). There are no reported studies on the epidemiology of work-related SCI in Africa or South Africa.

In South Africa, the Occupational Health and Safety Act (Act 85 of 1993) (hereafter referred to as OHSA) and the Mine Health and Safety Act (MHSA) (Act 29 of 1996) assert that the employer should "provide and maintain, as far as is reasonable and practicable a work environment that is safe and without risks to the health of employees. Section 8 (1) and 2 (a-j) of the OHSA, clearly stipulates the role of the employer in this regard: the provision and system of work should be safe and without risks to health of employees; to eliminate or mitigate any hazard or potential hazard to safety or health of employees before resorting to personal protective equipment (PPE); to ensure that all risks to health are absent during the process of work; to identify all hazards to health or safety of persons that might be attached to any work to be performed and to establish what precautionary measures should be taken with respect to such work and provide the necessary means to apply such precautionary measures; to provide information, training, supervision and instruction to employees regarding health and safety at work; not to permit employees to perform duties that are hazardous without appropriate precautions taken; to ensure that all necessary measures to ensure compliance to this act are in place and are enforced and to make sure that all equipment used by employees to perform their duties are safe." The MHSA protects employees in the mining sector, while the OHSA protects employees in other industrial sectors. Both acts were established to guide the industries and labour practitioners with the enforcement of health and safety measures in the workplace and to promote a culture of health and safety. However, they can only be effective if the following take place: monitoring and inspections; establishment of occupational health and safety committees (where both the employer and employee can demonstrate their level of interest and participation in issues of health and safety in the workplace); provision of occupational health facilities and occupational health and safety officials (who monitor the compliance of the employer and employee safety in the workplace) (OHSA, 1993). This can only happen if primary prevention is the focus in the management of health in the workplace (O'Connor, 2001).

The Compensation of Occupational Injuries and Diseases Act (Act 130 of 1993), was developed to provide compensation for individuals who sustain an injury or acquire a disease whilst performing the duties of his occupation. This act does not only makes provision for financial compensation to individuals, it also covers all medically-related costs. The Compensation Fund division of the Department of Labour is the unit that administers compensation claims related to individuals who have sustained or acquired work-related injury or disease. Funding of this unit comes from the National Budget. Funds that are allocated for this purpose can be shifted vertically or horizontally to other state priorities like education, health and job creation. 


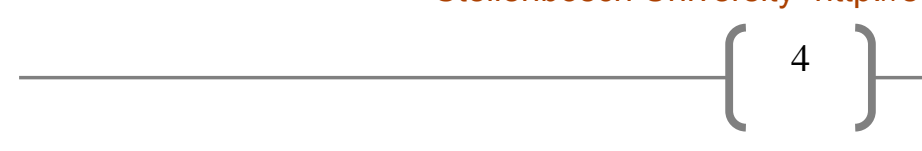

Although there is a good legal framework in South Africa that promotes health and safety in the workplace, there are no specific guidelines that seek to prevent SCI in the workplace, so SCIs continue to plague the workforce in every industry.

According to Jacobs and Jeebhay from the Department of Community Health UCT (n.d.), the employer and the state have a legal obligation, and a significant role, in the prevention of injuries in the workplace by means of integrated occupational health services. Currently, these services are fragmented and uncoordinated; only large companies have established occupational health services with a view to reducing absenteeism, thereby increasing productivity and also meeting legal requirements. Therefore, there is a need to explore prevention of SCIs to assist occupational health services to develop specific guidelines on the prevention of these devastating injuries.

There is a relative paucity of literature on the prevention of SCI in the workplace. Literature has mainly focused on prevention of SCI that occur during sporting activities (Silver and Stewart, 1994; Bhide, Edmonds and Tator, 2000; Barss, Djerrari, Leduc, Lepage and Dionne, 2008) motor vehicle accidents, occupational injuries (Feldstein, Breen and Dana, 1998) and injuries in general (Yeo and Walsh, 1987; Yeo, 1993). There is no specific literature on the prevention of work-related SCI in South Africa.

\subsection{Study Problem}

Currently, there is insufficient knowledge on the epidemiology of work-related SCI in South Africa to develop and implement preventative programmes. Data on the industry sectors that are more affected by work-related spinal cord injuries, their geographical distribution, activities that employees were engaged in when they sustained these injuries and compliance of employees and employers with the health and safety regulations in the workplace, are scarce.

However, this is the very information needed to embark on preventative programmes. Therefore the current study evolved.

\subsection{Aims Of The Study}

The aims of this study were to describe work-related SCI in Gauteng Province, South Africa, and to describe the compliance of employees and employers with the rules and regulations of the OHSA, to provide employers and policy makers with the necessary information to develop preventative strategies against SCI in the workplace. 


\subsection{Objectives Of The Study}

1. To describe the demographic profile of the study participants.

2. To determine the type and severity of the SCIs seen at the two study settings in Gauteng Province, South Africa.

3. To determine the causes and mechanisms of the work-related SCIs suffered by the participants.

4. To describe the nature of rehabilitation interventions received by individuals with SCI at the two study settings in Gauteng Province, South Africa.

5. To determine the distribution of these work-related SCIs with regards to geographical location and industry sectors.

6. To determine the average cost per person of rehabilitation intervention in work-related SCI during the study period.

7. To determine the impact of rehabilitation on individuals with SCI, seen at the two study settings in Gauteng Province, South Africa.

8. To determine the attitudes and practices of employees and employers regarding safety and injury prevention at work.

9. To provide recommendations on prevention of work-related SCI to employers, employees and government policy makers and implementers.

\subsection{Motivation Of The Study}

The researcher was employed by the Department of Labour (Compensation Commissioner) as a Claims Assessor Consultant. The job entailed assessing physiotherapy claims from the service providers of employees who sustained work-related injuries. A substantial number of these claims were claims of employees who sustained SCI in the workplace. The researcher developed an interest in this area, especially in the development of preventative mechanisms to prevent these injuries from occurring.

Currently, very little specific focus is being placed on preventing SCI in the workplace. Prevention of work-related SCI will save the state a lot of money through fewer compensation claims. These funds can be invested in preventative programmes which would benefit employees as well as employers, as fewer injuries will lead to increased production and some employees will be saved the immense trauma of having to deal with a SCI.

However, information on the size, distribution and causes of the problem are lacking, as is information on the impact of these injuries. Without this background information it is difficult, if not 


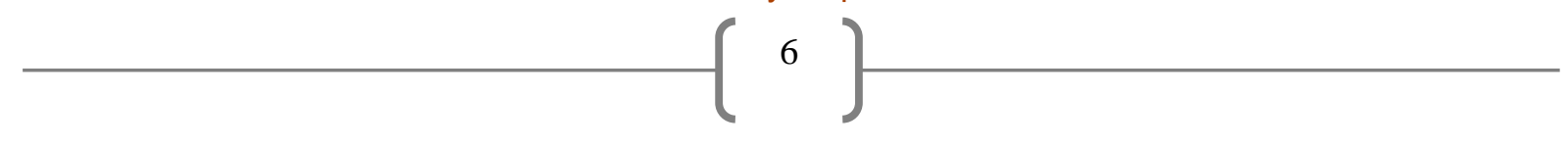

impossible, to develop relevant preventative mechanisms. Therefore, the researcher proposes to make a start towards addressing this lack in knowledge through this research.

\subsection{Conceptual Framework}

This study is based on four pillars to answer the specific objectives. This forms the framework for the study and is presented in Figure 1.1.

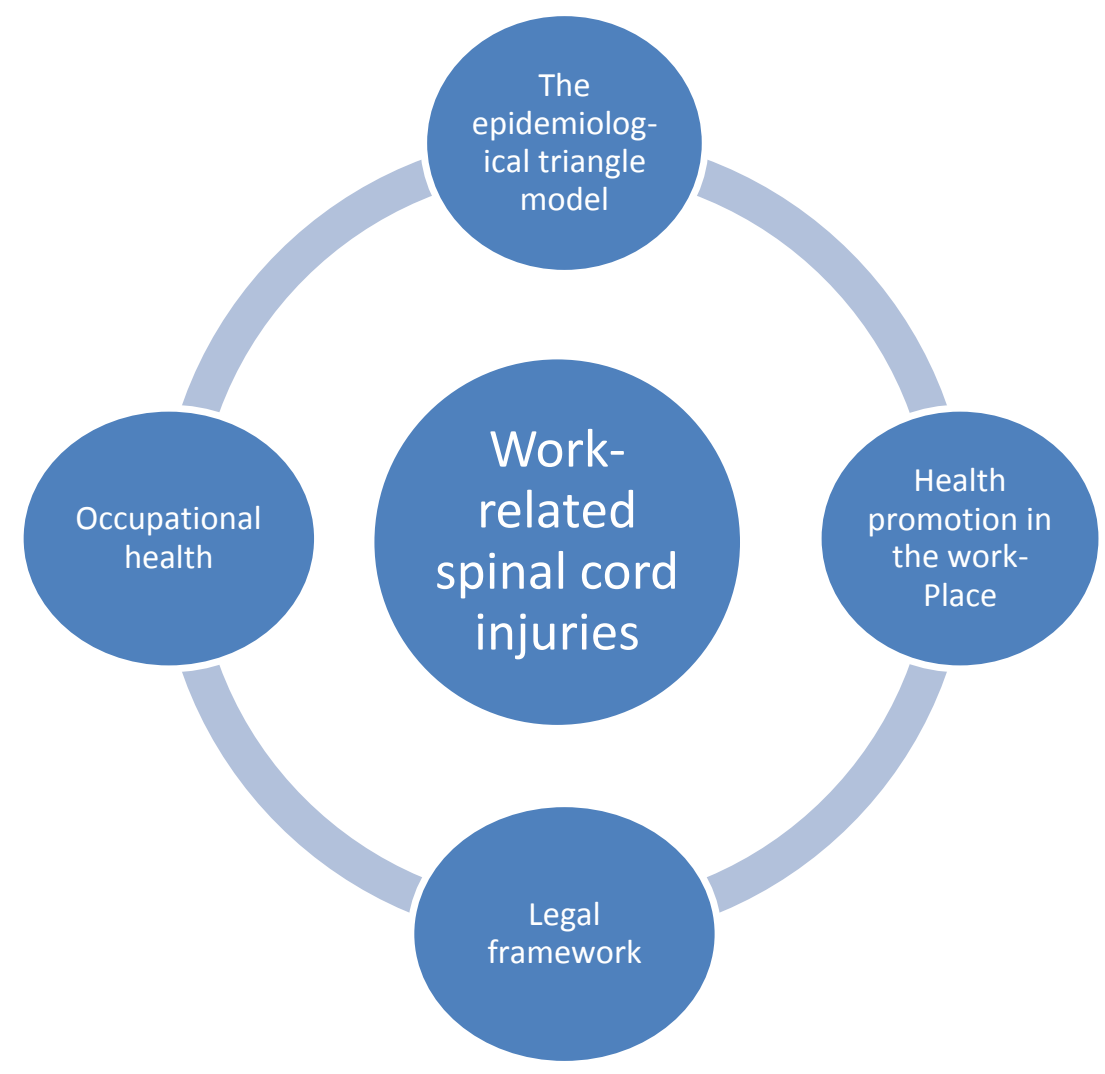

Figure1.1: Study Conceptual Framework

The first pillar of this study is the epidemiological approach adopted to describe work-related spinal cord injuries. The epidemiological triangle model is used to identify the injured person (host), agent or cause responsible for the injury (energy) and the environment in which these injuries occur in (Joubert, Ehrlich, Katzenellenbogen and Abdool Karim, 2007). The accumulation of epidemiological information makes the task of developing preventative strategies easier as the causes and patterns of injury are described.

The second pillar is occupational health. This aims to prevent workers from sustaining injuries or acquiring diseases as a result of exposure to hazards in the workplace. The principles underpinning Occupational Health Services in developing countries are to control workplace hazards, to prevent 
occupational ill health (e.g. back injury, lead poisoning, delayed fertility, asbestos-related diseases, silicosis and hearing loss) and occupational stress and to identify affected workers (Joubert, 2002).

Occupational health activities in each industry are critical in the prevention of work-related injuries. The researcher believes that evaluation of the existence of these activities is critical to develop a scope for rehabilitation professionals in terms of health promotion (behaviour modifications strategies) in an attempt to prevent the occurrence of SCI in the workplace.

The third pillar is health promotion in the workplace. Health promotion includes those activities embarked upon to improve the quality of one's life, thereby moving the individual forward toward a state of wellness or optimal health (Naidoo and Wills, 2009). Health care professionals with a special interest in occupational health are well positioned to pass on knowledge that would change attitudes and behaviours of employees. They are thus suitably qualified to plan, develop, implement and evaluate workplace health promotion interventions. Employee health promotion programs are based on the theory that it costs less to educate workers about controllable lifestyle health risks than to deal with the pricy costs (direct or indirect) of ill-health in the workplace (Naidoo and Wills, 2009).

The last pillar is the legal framework that provides the guidelines on the maintenance of health in the working environment and the compensation of workers, should they sustain a work-related injury or acquire a work-related disease. This framework guides industries to develop health and safety practices that seek to prevent workers from work-related hazards (Occupational Health and Safety Act,1993). Evaluation of the compliance of both the employee and employer with their obligations according to safety and health legislation is one component that will be evaluated in this study in an attempt to develop strategies that can be used to decrease SCI in the workplace.

\subsection{Significance Of The Study}

This descriptive study yielded information that can be used in the development and formulation of a preventative framework for work-related SCI in Gauteng Province, South Africa. Lessons learned from this study can also, with consideration of demographics, be applied to other provinces in South Africa, and to all industrial sectors. Currently, the only legal preventative framework is that drawn up by the OHSA, but more work needs to be done to draw up practical guidelines that will benefit all sectors in preventing these injuries.

The study will inform the Gauteng Department of Labour about the compliance of industries with the OHSA, policies and regulations in Gauteng Province. This could develop an interest in more research on this and related topics in other provinces in South Africa, to ensure more generalised compliance with the OHSA. 
This study represents a shift from the more common, rehabilitative approach to SCI, to an approach focussing on primary prevention of work-related spinal cord injuries. Through implementing the findings of the study, physiotherapists and other health professionals will have an opportunity to apply their health promotion skills in the workplace. A more meaningful role for the physiotherapist in occupational health will be created.

In addition no other study on the epidemiology of work-related SCI in South Africa or Africa could be found during the literature search. Therefore this study seems to be a first of its kind and findings will make a unique contribution to the body of knowledge on SCI in Africa.

\subsection{Outline Of The Study}

The nature of the study is epidemiological in that the researcher explores work-related injuries in the study setting. The study was conducted in two phases:

\subsubsection{Phase 1}

The first phase comprised the quantitative component, during which information from the two research sites' records was collected to develop a profile of work-related SCI with regard to causes and mechanisms, the distribution of injuries according to industries and type of employment, and the cost of rehabilitation. During this phase, the industries most commonly affected were identified and the qualitative phase of the study was conducted in these industries.

\subsubsection{Phase 2}

The qualitative phase were to focus on aspects like the knowledge of employees and employers on safety and injury prevention, the compliance of the identified industries with the OHSA, and identifying existing preventative measures in the industries. However, the researcher was unable to secure permission from the companies approached to perform this part of the study. Thus data could only be collected from persons who suffered an work related SCI. This substantially limited the findings and conclusions that could be drawn from them.

The study will be presented in detail in the following chapters:

- In Chapter 2, the literature review seeks to peruse all the relevant information about the issues that concern the study. Epidemiology of SCI will be reviewed and a comparison of data from both developed and developing countries will be made. Literature on the costs of SCI will also be reviewed. Patterns of these injuries in different occupational settings will be described. The South African legal framework on the promotion of health and safety in the 
workplace will be presented and issues of compliance with safety laws, guidelines and policies will be explored.

- Chapter 3 describes the methodology used in the study.

- Chapters 4 and 5 present the findings for both phases of the study.

- In Chapter 6, the results are discussed and compared with current literature on the subject.

- In Chapter 7, the conclusions of the study are given and the developed framework to prevent SCI in the study setting is presented. Recommendations will be made based on the conclusions of the study.

\subsection{Chapter Summary}

There is currently no cure for spinal cord injuries. A person sustaining a SCI has to undergo extensive medical treatment and rehabilitation at a very high cost. Prevention of these injuries in the workplace is crucial and in line with current legislation that requires companies to take reasonable measures to prevent injuries occurring in the workplace. The first step towards drawing up preventative strategies is to gather information about the magnitude and characteristics of the problem one wants to prevent. Thus this study evolved to gather information on work-related SCI in Gauteng Province, South Africa. 


\section{CHAPTER 2: LITERATURE REVIEW}

\subsection{Introduction}

The literature reviewed in this chapter is divided into two sections: viz. the literature relating to spinal cord injury (SCI) and that related to health and safety in the workplace. The SCI-related literature will cover information related to the definition and pathophysiology of SCIs and their effects. Epidemiology of SCI in Africa and world-wide will be described and compared. The chapter will explore work-related SCIs in relation to factors that contribute to the causes of SCIs in various environments.

Further, the economics of SCI in both developed and developing countries will be explored, including direct and indirect costs of SCI. Issues of disability (participation and integration) in people with SCI will be reviewed. Lessons learned from other countries on rehabilitation of SCIs worldwide will be explored, together with strategies of prevention of SCI in various environments, especially in the workplace.

The second part of this chapter will review literature pertaining to health and safety in the workplace. This will include a discussion on current models of prevention and management of injuries in the workplace, with specific reference to SCIs. Health and safety regulations in South Africa will be explored, as well as compliance of employees and employers with these.

\subsection{Introduction To Spinal Cord Injuries}

Spinal cord injury is a life transforming condition of sudden onset that can have devastating consequences (Paddison and Middleton, 2005). Depending on its severity, it may cause dramatic changes in the person's life (Umphred, 2007). The effects of SCI have a huge impact on the daily functioning of individuals and their families. This is especially true when the individual concerned is the bread winner in the family (Umphred, 2007).

The vertebrae (bones) that form the spinal column protect the encased soft tissues of the spinal cord. This spinal column can be fractured or dislocated in a number of different ways and cause traumatic injury to the spinal cord. Injuries to the cord can occur at any level of the spine (Somers, 2001). The term SCI is used to refer to structural damage of the cord, resulting in a change in the functioning of its motor, sensory and or autonomic functions, either temporary or permanent, below the level of the injury (Dumont, Okwonko, Verma, Hurlbert, Boulos, Ellegala and Dumont, 2001; Somers, 2001). 
To better understand the mechanisms of SCI and how the spine's functions are affected during an injury, a review of the anatomy and physiology of the spine and the spinal cord will be discussed below.

\subsubsection{Anatomy and physiology of the spinal cord}

The spinal cord travels within the vertebral canal of the spine and is important for transmission and integrating sensory and motor information between the brain and the somatic and visceral structures (Harvey, 2008). It extends from the medulla oblongata, just above the foramen magnum to the level of $1^{\text {st }}$ and $2^{\text {nd }}$ lumbar vertebrae. The grey matter is centrally located and is composed of cell bodies, small projection fibres and glial cells. Surrounding the grey matter is the white matter. The white matter consists of ascending and descending fibres and the axons of the motor and sensory neurons. These fibres carry sensory or motor functions and they travel together in tracts (Harvey, 2008). Table 2.1 explains the different types of sensory and motor tracts and their functions. The section thereafter will explain the consequences of injury to the spinal cord and how the functions of the structures are disturbed.

\begin{tabular}{|c|c|c|}
\hline Motor Tracts & Position & Functions \\
\hline Ventral cortico-spinal tract & Contralateral & Voluntary movement of the axial muscles \\
\hline Rubro-spinal tract & Ipsilateral & $\begin{array}{l}\text { Voluntary movement of the upper limb and precise } \\
\text { movement of distal muscles }\end{array}$ \\
\hline Vestibulo spinal tract & Bilateral & Posture and balance \\
\hline Lateral and medial reticulo spinal tract & Ipsilateral & $\begin{array}{l}\text { Posture, balance, spinal reflexes, axial and } \\
\text { proximal movements }\end{array}$ \\
\hline Lateral cortico-spinal tract & Ipsilateral & $\begin{array}{l}\text { Voluntary movement, precise controlled movement } \\
\text { of the distal limbs }\end{array}$ \\
\hline \multicolumn{3}{|l|}{ Sensory Tracts } \\
\hline $\begin{array}{l}\text { Spinothalamic, spinoreticular, } \\
\text { spinotectal tracts }\end{array}$ & Contralateral & Pain, temperature, crude touch \\
\hline Dorsal column & Ipsilateral & $\begin{array}{l}\text { Proprioception, vibration sense, deep touch and } \\
\text { discrimination touch }\end{array}$ \\
\hline Dorsal spinocerebellar & Ipsilateral & Proprioception from the trunk and lower limbs \\
\hline
\end{tabular}

Table 2.1 Motor and sensory tracts of the spinal cord 


\subsubsection{Pathophysiology of spinal cord injury}

It is important to have a basic understanding of the mechanisms that underlie the pathophysiology of SCI, to facilitate the decision-making process in choosing rehabilitation interventions. Spinal cord injury occurs when the cord sustains damage due to impingement by bony or soft tissue structures. The injury can occur when a vertebra dislocates, a vertebral body bursts or the cord is damaged by a foreign body (knife or bullet) (Somers, 2001).

Furthermore, Somers (2001) explains, the spinal cord does not have to be severed for irreversible damage to occur; SCI does not only occur because of physical trauma to the cord but can also happen because of disturbed vascular supply to an area of the cord. This can be caused by multiple factors including trauma, tumours, rheumatoid arthritis and haematomas. For the purposes of this study, only the pathology related to trauma will be explored.

The pathophysiology of traumatic SCIs is divided into a primary and secondary phase.

\subsubsection{Primary phase}

The primary phase is the initial phase in which the immediate response to the injury takes place.

The early mechanical trauma includes traction and compression. Direct compression of the cord by fractured and displaced bone fragments, disc material and ligaments injure both the central and peripheral nervous system (McDonald and Sadowsky, 2002). Four characteristic mechanisms of this phase have been identified: impact plus persistent compression; impact alone with transient compression; distraction; and laceration or transaction (Dumont et al., 2001). Blunt injuries (injuries that do not result in a severed cord) to the spinal cord occur as the osteo-ligamentous spinal column fails under different mechanical loading conditions including flexion, extension, axial load, rotation and distraction (Kwon, Tetzalaff, Grauer and Beiner, 2004). These forces impart the primary mechanical insult to the cord. In its mildest form it causes a cord concussion with temporary transient neurological deficits, and in its most rigorous form complex and permanent paralysis.

The degree of both initial tissue disruption and subsequent secondary injury is likely to be directly related to the transmission of energy delivered to the spinal cord (Kwon et al., 2004; Winter and Pattani, 2008). According to Dumont et al (2001), the initial trauma to the spinal cord tends to damage mostly the central grey matter, and the white matter (especially peripherally) is mostly less damaged. It is speculated that this phenomenon occurs as a consequence of the softer consistency and greater blood supply of the grey matter. Evidence exists that suggests that bleeding of the cord develops early following injury, and that blood circulation within the spinal cord is later disrupted following the 
injury. This leads to local neural tissue death caused by lack of oxygen and ischemia, predominantly in the grey matter (Dumont et al., 2001). This results in disruption in the functioning of the neurons.

The grey matter gets permanently damaged quicker (within an hour) post injury than the white matter (after 72 hours). This process leads to the secondary phase of the body's response to the injury.

\subsubsection{Secondary phase}

Most of the damage to the cord is caused by consequences that follow the initial trauma (Somers, 2001) or the secondary phase. The secondary phase is initiated by a multi-factorial process that expands the area of cell death and damage along the cord. According to Dumont et al (2001), the primary mechanical injury serves as the basis from which the secondary mechanisms of injury proceed. The latter include neurogenic shock, vascular insults, such as haemorrhage and ischemiareperfusion, excitotoxicity, calcium-mediated secondary injury and fluid-electrolyte disturbance, immunologic injury, apoptosis, disturbances in mitochondrial function and other processes (Sekhon and Fehlings, 2001; Dumont et al., 2001; McDonald and Sadowsky, 2002).

According to Kwon et al. (2004), local changes in blood flow and lack of oxygen within the spinal cord following an injury are the most important aspects of secondary injury. Following an injury to the spinal cord, hypo-perfusion develops and is profound in the grey matter and later progresses to the white matter. This slows or stops the transmission of the action potential along the axons resulting in spinal shock (MacDonald and Sadowsky, 2002). The disruption of the vascular system leads to loss of auto-regulatory mechanisms.

The loss of auto-regulation makes the cord vulnerable to systematic arterial pressure (Kwon et al., 2004). According to Sekhon and Fehlings (2001), there is good evidence that substantiates the role of free radicals in the secondary mechanism of SCI. Their role is thought to cause impairment of phospholipids-dependent enzymes, disruption of ionic gradients and membrane lysis, and decrease in adenosine tri-phosphate.

Collectively they induce metabolic failure and consequent necrotic or apoptotic death of a cell (cell suicide) (Kwon et al., 2004). Excitotoxicity is a process that includes the release of toxic chemicals by damaged cells, axons and blood vessels that assault the healthy adjacent cells (McDonald and Sadowsky, 2002). According to Dumont et al (2001), there is evidence that suggests that inflammatory and immunological response, following SCI, contributes to cell death in the spinal cord. Cell death in the spinal cord means that the functions of the motor and sensory tracts are affected. This leads to the loss of voluntary motor function, sensation, muscle tone, breathing and coughing, 
bowel and bladder functioning, genital functioning, cardio-vascular functioning and thermo-regulation (Somers, 2001).

\subsubsection{Classification of spinal cord injuries}

Spinal cord injuries are classified according to the level of the spine (paraplegia or tetraplegia) that is affected and whether the lesion is complete or incomplete (Umphred, 2007). Paraplegia refers to the injury or loss of motor and or sensory functions in the thoracic, lumbar or sacral segments of the spinal cord, following damage to the neural elements within the spinal canal. The use of the arms is spared while trunk and lower extremity function are impaired to a larger or lesser extent, depending on the level affected (Somers, 2001; Umphred, 2007; Bromley, 2006). Tetraplegia, or quadriplegia, refers to the injury or loss of motor and sensory function in the cervical segment of the spinal cord subsequent to damage of the neural structures within the spinal canal (Maynard, Bracken, Creasey, Ditunno, Donovan, Ducker, Garber, Marino Stover, Tator, Waters, Wilberger and Young, 1997). The functioning of the upper extremities, trunk and lower limbs are affected (Somers, 2001; Umphred, 2007; Bromley, 2006).

Completeness of the injury is determined by the degree to which motor and sensory functioning is preserved at a specific level of the spinal cord. A motor and sensory level refers to the most upper segment of the spinal cord with normal sensory or motor performance on both sides of the body. This is determined by testing for sensation alongside the dermatomes and testing for muscle strength (myotomes) on both sides of the body (Maynard et al., 1997).

An injury is defined as incomplete if partial continuation of sensory and or motor functions is found below the neurological level, including the lowest sacral segment (Maynard et al., 1997). A complete injury is where no motor or sensory performance is found below the neurological level. The completeness of the injury can be measured with the Frankel or American Spinal Cord Association (ASIA) scale (Maynard et al., 1997).

The Frankel scale was developed in the late 1960s and was widely adopted for use in the 1970s and 1980s because it was easy to use. However, it was not precise in the differentiation of grades C and D (Bromley, 2006). The ASIA scale was then developed in 1984 by adapting the Frankel scale (Maynard et al., 1997). Table 2.2 shows the differences in the two scales. The ASIA is currently widely used in clinical practice (Maynard et al., 1997) and was used in the current study to measure completeness of the injuries. 
Table 2.2 Scales used to classify SCI according to completeness of the injury

\section{ASIA Impairment Scale}

A: Complete: no sensory or motor level below S4-5

B: Incomplete: sensation preserved below the level of the lesion, including S4-5. No motor function.

C: Incomplete: motor function is preserved below the neurological level. More than half of the key muscles have a muscle grading of less than $3 / 5$.

D: Incomplete: motor function is preserved below the neurological level. At least half of the key muscles have a muscle grading of $3 / 5$.

E: Normal: sensory and motor function is normal

\section{Frankel Grading}

Complete

Incomplete: sensation preserved. No motor function.

Incomplete: preserved motor nonfunctional. Minimal voluntary motor function preserved.

Incomplete: preserved motor functional. Functional useful voluntary motor functioning preserved.

Complete return.

\subsubsection{Rehabilitation of spinal cord injuries}

According to Wilson and McLellan (1997), rehabilitation is a course of action aimed at enabling people with disabilities to reach and maintain their optimal physical, sensory, intellectual, psychological and social functional levels. Rehabilitation provides disabled people with the tools they need to reach independence and self-determination (Raine, Meadows, Lynch-Ellerington, 2009).

The primary goals of rehabilitation in individuals who sustained SCI are to prevent secondary complications, to maximise physical functioning and to reintegrate them into the community (Somers, 2001). According to Burns and Ditunno (2001) the care of patients with SCIs depends on the cooperation of the surgeons and the rehabilitation professionals. The surgical team focuses on preventing the development of the neurological deficits through the use of drugs, traction and surgical correction of structural instability of the spine and the structures that surrounds it.

The central function of the rehabilitation team (Table 2.3) is to assess and preserve the multiple organ systems affected by the SCI with the goal of restoring, substituting and/or modifying function and finally returning the patient to the community (Burns and Ditunno, 2001). Sinclair, Lingard and Mohabeer (2009) identified the following as the major contributors to effective inter-professional teams in rehabilitation: collaborative leadership, care philosophy (inter-professional vision statement), relationships (relationship pairs between physiotherapists and occupational therapists were observed), environmental context and structure of communication. According to Saulino (2011) rehabilitation 
following SCI is best delivered in a multidisciplinary, team-based approach. His description of the team members and their duties are presented in Table 2.3.

Table 2.3: Rehabilitation team members and their roles in SCI rehabilitation (adapted from Saulino, 2011)

\begin{tabular}{|l|l|}
\hline Rehabilitation Professional & Area of focus in Rehabilitation of SCI \\
\hline Physiotherapist & Focuses on lower limb function and on challenges with movement. \\
\hline Occupational Therapist & $\begin{array}{l}\text { Addresses upper limb dysfunction and challenges in activities of daily } \\
\text { living. }\end{array}$ \\
\hline Nurse & $\begin{array}{l}\text { Concerned with issues of the use of the bladder and bowel and skin } \\
\text { management. }\end{array}$ \\
\hline Psychologist & $\begin{array}{l}\text { Deals with emotional and behavioural concerns of the patient and with any } \\
\text { potential cognitive dysfunction. }\end{array}$ \\
\hline Speech and Language Pathologist & Addresses issues of communication and swallowing. \\
\hline Social Workers & $\begin{array}{l}\text { The most important crossing point between the rehabilitation team, the } \\
\text { patient, family, employer and healthcare funder. }\end{array}$ \\
\hline
\end{tabular}

There is no agreement as to the most effective team approach in rehabilitation (Bettger and Stineman, 2007). Literature reveals multidisciplinary and interdisciplinary approaches in the management of SCI (McKinley, Seel, Gadi and Tewksbury, 2001; Sand, Karlberg and Kreuter, 2006; Chan and Chan, 2005; Saulino and Vaccaro, 2009). The types of rehabilitation services offered at most spinal cord rehabilitation centres include medical rehabilitation, vocational rehabilitation, diet therapy, counselling and cognitive behavioural therapy (Harvey, 2008; Saulino, 2011). Cognitive behavioural therapy is offered to patients who sustained both SCI and head injury. Patients with dual diagnosis often present with cognitive impairments such as poor insight, problem solving, attention and memory problems (Harvey, 2008). In some instances, cognitive behavioural therapy can be used for patients who suffer from psychiatric symptoms (anxiety, depressed mood and poor self-esteem) subsequent to SCI (Craig, Hancock, Dickson and Chang 1997; North, 1999).

According to North (1999), the psychological effects following SCI are poorly understood but there is speculation that initially, the immediate reaction to the injury includes numbness, disbelief, anger, fear, hope and despair. This period is viewed as the period of turmoil. Counselling can be offered to persons with SCI to alleviate these symptoms and to make sure that the individual and the family are well adjusted. 
Diet therapy is offered to patients with SCI to reduce the risk of cardio-vascular diseases, prevent pressure sores and for bowel and bladder management (Somers, 2001). Medical rehabilitation is offered to prevent secondary complications and to maximise functional outcomes of an individual with SCI. The occurrences of secondary medical complications following SCI are a reflection of poor rehabilitation efforts (Somers, 2001). These medical complications may include the following: pneumonia/atelectasis, deep vein thrombosis, pulmonary embolus, pressure sores, autonomic dysreflexia, renal calculi, osteoporosis and fractures, heterotrophic ossification and pain (Somers, 2001; McKinley, Jackson, Cardenas and DeVivo, 1999).

These complications can lead to prolonged hospital stays thereby increasing the costs of rehabilitation of people with SCIs (McKinley et al., 1999). Rehabilitation efforts should therefore attempt to prevent these complications by making sure that the risk factors are controlled at all times, even after discharge. This means that there should be extensive collaboration with the family or caregivers and the rehabilitation facilities in the community, especially with primary healthcare facilities.

\subsubsection{Community integration of people with spinal cord injuries}

Community integration is an important goal of rehabilitation (Whiteneck et al,, 1999) and has been defined as a process of "acquiring or resuming age/gender/culture appropriate roles/statuses, including independence or interdependence in decision making and productive behaviours performed as part of multi-varied relationships with family, friends and others in natural community settings" (Dijkers, 1998:1485).

According to Whiteneck et al. (1999) community integration depends on the following factors: description and consequences of the injury or pathology, individual characteristics (demographics, lifestyle and behaviour) and the setting (physical, social, attitudinal and cultural issues). Their research showed that the best indicators of successful community integration were less rigorous neurological injuries, young (age), white ethnicity, higher levels of education and long periods post injury. Charlifue and Gerhart (2004) added high levels of life satisfaction, good physical health, social opportunities and low levels of stress.

\subsubsection{Spinal cord injury and employment}

Unemployment remains a problem in individuals with SCI compared with the general population (Ottomanelli and Lind, 2009). The authors explored various factors that influenced the employment rate of an individual with SCI: age, previous educational qualifications, environmental factors and personal factors. The authors identified that college level of education before and after sustaining SCI; being younger at time of injury; being white (race); greater physical ability following rehabilitation 
and being classified as paraplegia had a positive influence on employability of people with SCI following rehabilitation. They further argue that rehabilitation programmes should take into consideration these factors when implementing vocational rehabilitation services. Vocational rehabilitation services are rendered to persons with SCI in an attempt to integrate them back to their own occupations or alternative occupations. The main aim of vocational rehabilitation is to improve participation of individuals with SCI in the open labour market, thereby improving their opportunities of actively participating in the economy of the country. This improves their quality of life significantly.

\subsubsection{Outcome measures in spinal cord injury}

Evidence-based practice is the clear utilisation of current available evidence for clinical decision making in the process of managing patients (Iles and Davidson, 2006). There is a growing need for rehabilitation professionals (including physiotherapy) to show and provide evidence that indicate the effectiveness of interventions used during rehabilitation (Iles and Davidson, 2006).

This indicates the need to develop tools that are going to accurately measure rehabilitation outcomes at different levels. The International Classification of Function, Disability and Health (ICF) provides a framework to guide rehabilitation professionals in managing individuals with SCI (Harvey, 2008). It assists in assessing impairments, activity limitations and participation restriction in relation to contextual factors. Stucki (2005) sees it as providing a pathway that can be used by clinicians to explore and find the relationship between the components. For an example: how do the impairments affect the level of functioning; how does the level of functioning affect the level of participation; and how do the environment and personal factors influence participation levels? This is done by evaluating each component and exploring the effect it has in relation to the other components. A variety of outcome measures can be used to measure each component of the ICF. Table 2.4 summarises the list of outcome measurement tools that are commonly used to assess the magnitude of the limitations or difficulties experienced by people with SCI, and to assess the effects of rehabilitation interventions and to provide information regarding which aspect of the ICF is addressed. The ICF checklist is a short version of the ICF that can be used to assess the ICF components from written records (Stucki, 2005).

There is a specific ICF checklist that was developed for individuals who sustained SCIs (Kirchberger, Cieza, Biering-Sørensen, Baumberger, Charlifue, Post, Campbell, Kovindha, Ring, Sinnott, Kostanjsek and Stucki, 2009). The FIMAM is the most widely used outcome measurement tool to measure functional limitations in people with SCI (Somers, 2005; Rauch, Escorpizo, Riddle, EriksHoogland, Stucki and Cieza, 2010; Rauch, Baumberger, Moise, von Elm and Reinhardt, 2011). 
Table 2.4 Outcome measures in SCI rehabilitation

\begin{tabular}{|l|l|l|l|}
\hline Impairment measures & $\begin{array}{l}\text { Activity limitation } \\
\text { measures }\end{array}$ & $\begin{array}{l}\text { Participation } \\
\text { measures }\end{array}$ & $\begin{array}{l}\text { Measures of } \\
\text { environmental factors }\end{array}$ \\
\hline $\begin{array}{l}\text { American Spinal Injury } \\
\text { Association Scale (ASIA) }\end{array}$ & SCM & $\begin{array}{l}\text { Craig Handicap } \\
\text { Assessment and } \\
\text { Reporting Technique } \\
\text { (CHART) }\end{array}$ & $\begin{array}{l}\text { Craig Hospital Inventory } \\
\text { of Environmental Factors } \\
\text { (CHIEF) }\end{array}$ \\
\hline Frankel Grading Scale & $\begin{array}{l}\text { Functional Independence } \\
\text { Measure and Functional } \\
\text { Assessment Measure } \\
\text { (FIM+FAM) }\end{array}$ & SF-36 (QOL) & ICF \\
\hline $\begin{array}{l}\text { Medical Research } \\
\text { Council Muscle Grading } \\
\text { Scale }\end{array}$ & Barthel Index (BI) & $\begin{array}{l}\text { London Handicap Scale } \\
\text { (LHS) }\end{array}$ & \\
\hline Modified Ashworth Scale & $\begin{array}{l}\text { Walking Index for SCIs } \\
\text { (WISCI) }\end{array}$ & $\begin{array}{l}\text { Independent Living } \\
\text { Services Evaluation } \\
\text { (ILSE) }\end{array}$ & \\
\hline $\begin{array}{l}\text { Fatigue Severity Scale } \\
\text { (FSC) }\end{array}$ & & $\begin{array}{l}\text { Index of Psycho-Social } \\
\text { Well Being Index } \\
\text { (IPSWBI) }\end{array}$ & \\
\hline $\begin{array}{l}\text { Perceived Stress Scale } \\
\text { (PSS) }\end{array}$ & & $\begin{array}{l}\text { Personal Independence } \\
\text { Profile (PIP) }\end{array}$ & \\
\hline $\begin{array}{l}\text { Centre for } \\
\text { Epidemiological Studies } \\
\text { Depression Scale (CES- }\end{array}$ & Scate (SWLS) & \\
\hline
\end{tabular}

\subsection{Epidemiology Of Spinal Cord Injuries}

The researcher reviewed 33 studies on epidemiology of SCIs around the world. Out of these, 18 were conducted in Africa, and of these, six were conducted in South Africa. Only four studies worldwide were on work-related SCIs. Some of these studies were completed over a decade or more ago and the researcher realised that information might be dated. However findings from the older studies were included since they can provide information of changes and trends over time.

Studies conducted in developed countries included Australia, Canada, Greece, Ireland, Italy, Sweden and the Unites States of America (USA). Literature findings in this section will be presented through 
comparative tables and some discussion based on the information in the tables. Work-related SCIs will be discussed first, followed by non-work-related studies on SCIs in Africa and around the world.

\subsubsection{Epidemiology Of Work-Related Spinal Cord Injuries}

The four studies in this section span nearly four decades, with the most recent having been done nearly a decade ago in 2004. Table 2.5 summarises the studies published on work-related SCIs by number of participants, age, gender, type of study and the study period. The total number of participants was 544 with an average of 136 per study. The male-to-female ratio varied from 11.5:1 to $24: 1$, indicating that many more males than females were affected. However, the ratio decreased over the decades from 24:1 in 1985 to $11.5: 1$ in 2004 . The majority of participants in these studies are in their twenties, thirties or forties, thus, a relatively young cohort with many productive years ahead of them.

Table 2.5: Methodology and demographic findings from studies on Work-related SCIs

\begin{tabular}{|c|c|c|c|c|c|c|}
\hline Author(s) & $\begin{array}{l}\text { No. of } \\
\text { participants }\end{array}$ & $\begin{array}{l}\text { Male-to- } \\
\text { female } \\
\text { ratio }\end{array}$ & $\begin{array}{l}\text { Mean } \\
\text { age }\end{array}$ & $\begin{array}{l}\text { Most } \\
\text { affected } \\
\text { age } \\
\text { ranges }\end{array}$ & $\begin{array}{l}\text { Type of } \\
\text { study and } \\
\text { data source }\end{array}$ & $\begin{array}{l}\text { Study } \\
\text { period }\end{array}$ \\
\hline $\begin{array}{l}\text { Ekong and Tator, } \\
\text { 1985, (Canada) }\end{array}$ & 25 & $24: 1$ & NM & $20-40$ & $\begin{array}{l}\text { Retrospective; } \\
\text { Hospital } \\
\text { records }\end{array}$ & 5 years \\
\hline $\begin{array}{l}\text { Rosenberg et al., } \\
\text { 1993, (USA) }\end{array}$ & 79 & 19:1 & $\begin{array}{l}38.9 \\
\mathrm{NM}\end{array}$ & $16-45$ & $\begin{array}{l}\text { Retrospective; } \\
\text { Surveillance } \\
\text { database, }\end{array}$ & $\begin{array}{l}\text { 5years six } \\
\text { months }\end{array}$ \\
\hline $\begin{array}{l}\text { O’Connor, } 2001 \\
\text { (Australia) }\end{array}$ & 378 & 19:1 & NM & $24-54$ & $\begin{array}{l}\text { Retrospective; } \\
\text { National data } \\
\text { base (ASCIR) }\end{array}$ & 11 years \\
\hline $\begin{array}{l}\text { Webster et al., } \\
\text { 2004, (USA) }\end{array}$ & $\begin{array}{l}62 \\
\text { (only cervical } \\
\text { lesions) }\end{array}$ & $11.5: 1$ & $\begin{array}{l}38.7 \\
17-77\end{array}$ & NM & $\begin{array}{l}\text { Retrospective; } \\
\text { Workers } \\
\text { compensation } \\
\text { database }\end{array}$ & 11 years \\
\hline
\end{tabular}

Note: Not mentioned (NM) 


\subsubsection{Causes of work-related spinal cord injuries}

Table 2.6 summarises the causes of work-related SCIs. Falls were the major cause of work-related SCIs in all four studies, followed by road traffic accidents and being struck or crushed by an object.

Table 2.6: Causes of Work-Related SCIs

\begin{tabular}{|l|l|l|l|l|l|}
\hline Author(s) & $\begin{array}{l}\text { Road traffic } \\
\text { accidents }\end{array}$ & Falls & $\begin{array}{l}\text { Violence } \\
\text { gunshot and } \\
\text { stabbing) }\end{array}$ & $\begin{array}{l}\text { Struck or } \\
\text { crushed by } \\
\text { an object }\end{array}$ & Other \\
\hline $\begin{array}{l}\text { Ekong \& Tator, } \\
1985, \text { (Canada) }\end{array}$ & $12 \%$ & $56 \%$ & $0 \%$ & $32 \%$ & $0 \%$ \\
\hline $\begin{array}{l}\text { Rosenberg et al., } \\
1993, \text { (USA) }\end{array}$ & $19 \%$ & $50 \%$ & $5 \%$ & $19 \%$ & $7 \%$ \\
\hline $\begin{array}{l}\text { O'Connor, 2001, } \\
\text { (Australia) }\end{array}$ & $25 \%$ & $47 \%$ & $1 \%$ & $24 \%$ & $3 \%$ \\
\hline $\begin{array}{l}\text { Webster et al., } \\
\text { 2004, (USA) }\end{array}$ & $34 \%$ & $36 \%$ & $3 \%$ & $21 \%$ & $6 \%$ \\
\hline
\end{tabular}

\subsubsection{Classification of work-related spinal cord injuries}

Table 2.7 provides information on the level of work-related SCIs and their severity. The majority of the injuries were incomplete (ASIA B to E).

Table 2.7: Neurological Level and Severity of Work-Related SCIs

\begin{tabular}{|l|l|l|l|l|l|}
\hline Author(s) & Cervical & Thoracic & $\begin{array}{l}\text { Lumbar and } \\
\text { Sacral }\end{array}$ & $\begin{array}{l}\text { Complete } \\
\text { (ASIA A) }\end{array}$ & $\begin{array}{l}\text { Incomplete } \\
\text { (ASIA B-E) }\end{array}$ \\
\hline $\begin{array}{l}\text { Ekong \& Tator, } \\
1985, \text { (Canada) }\end{array}$ & $44 \%$ & $36 \%$ & $20 \%$ & $24 \%$ & $76 \%$ \\
\hline $\begin{array}{l}\text { Rosenberg et al., } \\
1993, \text { USA) }\end{array}$ & $45 \%$ & $31 \%$ & $24 \%$ & $42 \%$ & $58 \%$ \\
\hline $\begin{array}{l}\text { O'Connor, 2001 } \\
\text { (Australia) }\end{array}$ & $33 \%$ & $43 \%$ & $24 \%$ & $44 \%$ & $56 \%$ \\
\hline $\begin{array}{l}\text { Webster et al., } \\
\text { 2004, (USA) }\end{array}$ & $\begin{array}{l}\text { All } \\
\text { cervical }\end{array}$ & NA & NA & $47 \%$ & $53 \%$ \\
\hline
\end{tabular}

\subsubsection{Occupations and types of industries affected}

The most common occupational categories affected by work-related SCIs in the USA were construction, manufacturing, service, professional or managerial, transportation and retail and agriculture (Rosenberg et al., 1993; Webster et al., 2004). By contrast the industries most commonly affected in Canada were industrial (48\%), logging (8\%), construction (16\%), factory (8\%) and 
farming (8\%) (Ekong and Tator, 1985). Occupation categories most affected were labourers in farming, construction and mining. The estimated labour-based rate was highest in the farming industry (O'Connor, 2001). These findings are in contrast to the studies done in other developed countries, where farming was the least affected industry and construction was the leading cause of SCI.

Only one of the above studies on work-related SCIs reported on mortality rates: Ekong and Tator (1985) reported an in-hospital mortality rate of eight percent. Length of stay (LOS) and secondary medical complications were not reported on in any of the studies.

\subsubsection{Epidemiology of spinal cord injuries in Africa}

Studies conducted in Nigeria, South Africa, Zimbabwe, Tanzania and Sierra Leone, over the last four decades, were identified and will be discussed in this section. Table 2.8 (next page) summarises the number of participants, age, gender, the design and the setting of the study as well as the period investigated.

The number of participants in African studies ranged from 14 to 616. All but one of the studies was retrospective. According to Hess (2004) retrospective studies are inexpensive, use existing data records, and allow study of rare occurrences. These could be some of the reasons that caused the majority of African researchers to use this study design, although a prospective study design is preferable as the current exposure of the diseases or injury is observed (Joubert et al., 2007). The number of years for which data was gathered ranged from 1-14.

All of the studies were hospital-based which could be an indication that there are no active surveillance systems or SCI registers in Africa. The age range of the participants was between 10 and 80 years, with the most affected age range being 15-40. The male-to-female ratios varied between 2.1:1 to 11.5:1, where ratios of more than 7:1 were observed in Nigeria and Sierra Leone. Thus, the age ranges were similar to those of studies on work-related SCIs in developed countries, while the male-to-female ratios were lower. 
Table 2.8 Age and gender analysis on SCIs in Africa

\begin{tabular}{|c|c|c|c|c|c|c|}
\hline Author (s) & $\begin{array}{l}\text { No. of } \\
\text { participants }\end{array}$ & $\begin{array}{l}\text { Male- } \\
\text { to- } \\
\text { female } \\
\text { ratio }\end{array}$ & $\begin{array}{l}\text { Mean } \\
\text { age }\end{array}$ & $\begin{array}{l}\text { Most } \\
\text { affected } \\
\text { age } \\
\text { ranges }\end{array}$ & $\begin{array}{l}\text { Type of study } \\
\text { and data } \\
\text { source }\end{array}$ & $\begin{array}{l}\text { Study } \\
\text { period }\end{array}$ \\
\hline $\begin{array}{l}\text { Iwegbu, 1983, } \\
\text { (Nigeria) }\end{array}$ & $\begin{array}{l}48 \\
\text { Only } \\
\text { paraplegia }\end{array}$ & $7: 1$ & 2 & NM & $\begin{array}{l}\text { Retrospective; } \\
\text { Tertiary hospital }\end{array}$ & 9 years \\
\hline $\begin{array}{l}\text { Odendaal, 1991a, } \\
\text { (RSA: Gauteng) }\end{array}$ & $\begin{array}{l}40 \\
\text { Cervical } \\
\text { Injuries only, }\end{array}$ & $4,71: 1$ & 36.9 & NM & $\begin{array}{l}\text { Retrospective; } \\
\text { Public spinal unit }\end{array}$ & 5 years \\
\hline $\begin{array}{l}\text { Odendaal, 1991b, } \\
\text { (RSA: Gauteng) }\end{array}$ & $\begin{array}{l}48 \\
\text { Thoracic and } \\
\text { lumbar } \\
\text { injuries }\end{array}$ & $4,34: 1$ & 30.2 & NM & $\begin{array}{l}\text { Retrospective; } \\
\text { Public spinal unit }\end{array}$ & 5 years \\
\hline $\begin{array}{l}\text { Couto \& Levy, 1994, } \\
\text { (Zimbabwe) }\end{array}$ & 22 & & 31 & NM & $\begin{array}{l}\text { Retrospective; } \\
\text { Hospital records }\end{array}$ & NM \\
\hline $\begin{array}{l}\text { Hart \& Williams, } \\
\text { 1994, (RSA: Gauteng ) }\end{array}$ & 616 & $4: 1$ & NM & $15-39$ & $\begin{array}{l}\text { Retrospective; } \\
\text { Public spinal unit }\end{array}$ & 6 years \\
\hline $\begin{array}{l}\text { Velmahos et al., 1995, } \\
\text { (RSA: Gauteng) }\end{array}$ & 551 & $3.47: 1$ & 28 & $15-30$ & $\begin{array}{l}\text { Retrospective; } \\
\text { Public spinal unit }\end{array}$ & 5 years \\
\hline $\begin{array}{l}\text { Levy et al., 1998, } \\
\text { (Zimbabwe) }\end{array}$ & 136 & 7.3:1 & NM & $20-40$ & $\begin{array}{l}\text { Retrospective; } \\
\text { Tertiary hospital }\end{array}$ & 7 years \\
\hline $\begin{array}{l}\text { Igun et al., 1999, } \\
\text { (Nigeria) }\end{array}$ & 68 & $10: 1$ & 30 & $21-40$ & $\begin{array}{l}\text { Retrospective; } \\
\text { Tertiary hospital }\end{array}$ & 14 years \\
\hline $\begin{array}{l}\text { Solagberu, 2002, } \\
\text { (Nigeria) }\end{array}$ & 39 & $11.5: 1$ & 37.3 & NM & $\begin{array}{l}\text { Retrospective; } \\
\text { Tertiary hospital }\end{array}$ & 5 years \\
\hline $\begin{array}{l}\text { Le Roux \& Dunn, } \\
\text { 2005, (RSA: Western } \\
\text { Cape) }\end{array}$ & 49 & $3.46: 1$ & 27.5 & NM & $\begin{array}{l}\text { Retrospective; } \\
\text { Tertiary hospital }\end{array}$ & 1 year \\
\hline $\begin{array}{l}\text { Olasode et al., 2006, } \\
\text { (Nigeria) }\end{array}$ & 71 & $2: 1$ & NM & $3^{\text {rd }}$ and $5^{\text {th }}$ & $\begin{array}{l}\text { Retrospective; } \\
\text { Tertiary hospital }\end{array}$ & $\begin{array}{l}1 \text { year. } \\
6 \text { months }\end{array}$ \\
\hline $\begin{array}{l}\text { Frielingsdorf \& Dunn, } \\
\text { 2007, (RSA: Western } \\
\text { Cape) }\end{array}$ & $\begin{array}{l}101 \\
\text { Cervical } \\
\text { injuries only }\end{array}$ & $4.8: 1$ & 34.69 & NM & $\begin{array}{l}\text { Retrospective; } \\
\text { Public tertiary } \\
\text { hospital }\end{array}$ & 1 year \\
\hline $\begin{array}{l}\text { Udosen et al., 2007, } \\
\text { (Nigeria) }\end{array}$ & 14 & $4: 1$ & NM & $21-30$ & $\begin{array}{l}\text { Retrospective; } \\
\text { Tertiary hospital }\end{array}$ & 1 year \\
\hline $\begin{array}{l}\text { Emujulu et al., 2009, } \\
\text { (Nigeria) }\end{array}$ & 62 & $3.1: 1$ & NM & $0-40$ & $\begin{array}{l}\text { Prospective; } \\
\text { Tertiary hospital }\end{array}$ & 2 years \\
\hline $\begin{array}{l}\text { Obalum et al., } 2009 \\
\text { (Nigeria) }\end{array}$ & 468 & $2.34: 1$ & NM & $21-30$ & $\begin{array}{l}\text { Retrospective; } \\
\text { Tertiary hospital }\end{array}$ & 15 years \\
\hline $\begin{array}{l}\text { Gosselin \& Coppotelli, } \\
\text { 2010, (Sierra Leone) }\end{array}$ & 24 & $11.5: 1$ & 3 & NM & $\begin{array}{l}\text { Retrospective; } \\
\text { Hospital records }\end{array}$ & $\begin{array}{l}1 \text { year } \\
6 \text { months }\end{array}$ \\
\hline
\end{tabular}

Note: Not mentioned (NM) 
Table 2.9 (next page) summarises the causes of SCIs in Africa. The three major causes of SCI in Africa were road traffic accidents, falls and violence (including gunshot wounds, stabbings and assaults). In all countries but South Africa road traffic accidents were the most common cause of SCI, throughout the periods studied. In South Africa during the early 1980s road traffic accidents were the highest cause of SCIs but during the 1990s violence became the most common cause of SCIs, a trend that reverts to road traffic accidents from 2000 again. This changing picture could be due to the changing political climate in South Africa and especially violent period in the early nineties (Hart and Williams, 1994; Velmahos, Degiannis, Hart, Souter and Saadia, 1995).

Table 2.10 (page following Table 2.9) summarises the neurological level of the participants with SCI in Africa. The majority of the injuries were classified as complete lesions. The most affected region of the spine was the thoracic and the lumbar spine (paraplegia), although in some cases in Nigeria, SCIs affecting the cervical region of the spine were most represented (Emujulu, Ekweogwu, Nottidge, 2009; Obalum, Giwa, Adekoya-Cole, and Enweluzo, 2009; Olasode, Komalafe, Komalafe, and Olasode, 2006, Solagberu, 2002). 
Table 2.9: Causes of SCI in Africa

\begin{tabular}{|c|c|c|c|c|c|}
\hline Author(s) & $\begin{array}{l}\text { Road } \\
\text { traffic } \\
\text { accidents }\end{array}$ & Falls & $\begin{array}{l}\text { Violence } \\
\text { (gunshot } \\
\text { and stab } \\
\text { wounds ) }\end{array}$ & Sport & $\begin{array}{l}\text { Domestic or } \\
\text { occupational } \\
\text { related }\end{array}$ \\
\hline $\begin{array}{l}\text { Iwegbu, 1983, } \\
\text { (Nigeria) }\end{array}$ & $75 \%$ & $8 \%$ & $0 \%$ & $0 \%$ & $17 \%$ \\
\hline $\begin{array}{l}\text { Odendaal, 1991a, } \\
\text { (RSA: Gauteng) }\end{array}$ & $60 \%$ & $28 \%$ & $8 \%$ & $0 \%$ & $4 \%$ \\
\hline $\begin{array}{l}\text { Odendaal, 1991b, } \\
\text { (RSA: Gauteng) }\end{array}$ & $63 \%$ & $21 \%$ & $7 \%$ & $0 \%$ & $9 \%$ \\
\hline $\begin{array}{l}\text { Couto \& Levy, 1994, } \\
\text { (Zimbabwe) }\end{array}$ & $41 \%$ & $35 \%$ & $11 \%$ & $3 \%$ & $10 \%$ \\
\hline $\begin{array}{l}\text { Hart \& Williams, } \\
\text { 1994, (RSA: Gauteng) }\end{array}$ & $25 \%$ & $3 \%$ & $56 \%$ & $0 \%$ & $16 \%$ \\
\hline $\begin{array}{l}\text { Velmahos et al., 1995, } \\
\text { (RSA: Gauteng) }\end{array}$ & $30 \%$ & $0 \%$ & $61 \%$ & $0 \%$ & $9 \%$ \\
\hline $\begin{array}{l}\text { Levy et al., 1998, } \\
\text { (Zimbabwe) }\end{array}$ & $59 \%$ & $11 \%$ & $14 \%$ & $0 \%$ & $16 \%$ \\
\hline $\begin{array}{l}\text { Igun et al., 1999, } \\
\text { (Nigeria) }\end{array}$ & $57 \%$ & $22 \%$ & $3 \%$ & 0 & $18 \%$ \\
\hline $\begin{array}{l}\text { Solagberu, 2002, } \\
\text { (Nigeria) }\end{array}$ & $67 \%$ & $23 \%$ & $0 \%$ & $0 \%$ & $10 \%$ \\
\hline $\begin{array}{l}\text { Le Roux \& Dunn, } \\
\text { 2005, (RSA: Western } \\
\text { Cape) }\end{array}$ & $0 \%$ & $\begin{array}{l}\text { All were } \\
\text { Gunshot } \\
\text { wounds }\end{array}$ & $0 \%$ & $0 \%$ & $0 \%$ \\
\hline $\begin{array}{l}\text { Olasode et al., 2006, } \\
\text { (Nigeria) }\end{array}$ & $90 \%$ & $0 \%$ & $4 \%$ & $0 \%$ & $6 \%$ \\
\hline $\begin{array}{l}\text { Frielingsdorf \& Dunn, } \\
\text { 2007, (RSA: Western } \\
\text { Cape) }\end{array}$ & $55 \%$ & $16 \%$ & $21 \%$ & $8 \%$ & $0 \%$ \\
\hline $\begin{array}{l}\text { Udosen et al., } 2007 \text {, } \\
\text { (Nigeria) }\end{array}$ & $86 \%$ & $7 \%$ & $7 \%$ & $0 \%$ & $0 \%$ \\
\hline $\begin{array}{l}\text { Emujulu et al., 2009, } \\
\text { (Nigeria) }\end{array}$ & $60 \%$ & $31 \%$ & $0 \%$ & $0 \%$ & $9 \%$ \\
\hline $\begin{array}{l}\text { Obalum et al., 2009, } \\
\text { (Nigeria) }\end{array}$ & $77 \%$ & $9 \%$ & $9 \%$ & 0 & $5 \%$ \\
\hline $\begin{array}{l}\text { Gosselin \& Coppotelli, } \\
\text { 2010, (Sierra Leone) }\end{array}$ & ND & ND & ND & ND & ND \\
\hline
\end{tabular}

Note: No data (ND) 
Table 2.10 Classification of SCIs in Africa

\begin{tabular}{|c|c|c|c|c|c|}
\hline Author(s) & Cervical & Thoracic & $\begin{array}{l}\text { Lumbar } \\
\text { and } \\
\text { sacral }\end{array}$ & $\begin{array}{l}\text { Complete } \\
\text { (ASIA A) }\end{array}$ & $\begin{array}{l}\text { Incomplete } \\
\text { ASIA B-E) }\end{array}$ \\
\hline Iwegbu, 1983, (Nigeria) & & $\begin{array}{l}\text { Paraplegia } \\
\text { Only }\end{array}$ & & $88 \%$ & $12 \%$ \\
\hline $\begin{array}{l}\text { Odendaal, 1991a, } \\
\text { (RSA: Gauteng) }\end{array}$ & $\begin{array}{l}\text { Cervical } \\
\text { only }\end{array}$ & $0 \%$ & $0 \%$ & $20 \%$ & $80 \%$ \\
\hline $\begin{array}{l}\text { Odendaal, 1991b, } \\
\text { (RSA: Gauteng) }\end{array}$ & & $67 \%$ & $33 \%$ & $63 \%$ & $37 \%$ \\
\hline $\begin{array}{l}\text { Couto \& Levy, 1994, } \\
\text { (Zimbabwe) }\end{array}$ & $60 \%$ & $19 \%$ & $21 \%$ & $60 \%$ & $40 \%$ \\
\hline $\begin{array}{l}\text { Hart \& Williams, 1994, } \\
\text { (RSA: Gauteng) }\end{array}$ & $25 \%$ & $63 \%$ & $12 \%$ & $66 \%$ & $34 \%$ \\
\hline $\begin{array}{l}\text { Velmahos et al., 1995, } \\
\text { (RSA: Gauteng } \\
\text { Province) }\end{array}$ & $26 \%$ & $59 \%$ & $15 \%$ & $0 \%$ & $0 \%$ \\
\hline $\begin{array}{l}\text { Levy et al., 1998, } \\
\text { (Zimbabwe) }\end{array}$ & $0 \%$ & $0 \%$ & $0 \%$ & $0 \%$ & $0 \%$ \\
\hline $\begin{array}{l}\text { Igun et al., 1999, } \\
\text { (Nigeria) }\end{array}$ & $0 \%$ & $0 \%$ & $0 \%$ & $43 \%$ & $57 \%$ \\
\hline $\begin{array}{l}\text { Solagberu, 2002, } \\
\text { (Nigeria) }\end{array}$ & $46 \%$ & $41 \%$ & $13 \%$ & $0 \%$ & $0 \%$ \\
\hline $\begin{array}{l}\text { Le Roux \& Dunn, } \\
\text { 2005, (RSA, Western } \\
\text { Cape) }\end{array}$ & $26 \%$ & $47 \%$ & $27 \%$ & $78 \%$ & $22 \%$ \\
\hline $\begin{array}{l}\text { Olasode et al., 2006, } \\
\text { (Nigeria) }\end{array}$ & $55 \%$ & $\begin{array}{l}18 \% \\
\text { Thoracic and } \\
\text { Lumbar }\end{array}$ & $0 \%$ & $0 \%$ & $0 \%$ \\
\hline $\begin{array}{l}\text { Frielingsdorf \& Dunn, } \\
\text { 2007, (RSA, Western } \\
\text { Cape) }\end{array}$ & $\begin{array}{l}\text { Cervical } \\
\text { Only }\end{array}$ & $0 \%$ & $0 \%$ & $35 \%$ & $65 \%$ \\
\hline $\begin{array}{l}\text { Udosen et al., 2007, } \\
\text { (Nigeria) }\end{array}$ & $14 \%$ & $\begin{array}{l}86 \% \\
\text { Thoracic and } \\
\text { Lumbar }\end{array}$ & 0 & $71 \%$ & $29 \%$ \\
\hline $\begin{array}{l}\text { Emujulu et al., 2009, } \\
\text { (Nigeria) }\end{array}$ & $70 \%$ & $20 \%$ & $10 \%$ & $39 \%$ & $36 \%$ \\
\hline $\begin{array}{l}\text { Obalum et al., 2009, } \\
\text { (Nigeria) }\end{array}$ & $72 \%$ & $21 \%$ & $7 \%$ & $92 \%$ & $8 \%$ \\
\hline $\begin{array}{l}\text { Gosselin \& Coppotelli,, } \\
\text { 2010, (Sierra Leone) }\end{array}$ & $29 \%$ & $\begin{array}{l}71 \% \text { Both } \\
\text { Thoracic and } \\
\text { Lumbar }\end{array}$ & 0 & $82 \%$ & $18 \%$ \\
\hline
\end{tabular}


Table 2.11 summarises the length of stay (LOS), mortality rates and complications amongst participants with SCIs in Africa. The average LOS in Africa ranged between 30 and 153 days. The overall range was from 2-530 days. In South Africa LOS was reduced by surgical interventions (Odendaal, 1991a; Odendaal, 1991b). Nigeria had the widest range of LOS, between 1-530 days (Solagberu, 2002).

The mortality rate ranged between $2 \%$ and $50 \%$. The lowest was achieved in South Africa (Le Roux and Dunn, 2005) and the highest in Zimbabwe (Couto et al., 1994). The differences in the mortality rates could be due to the differences in the quality of healthcare interventions in both countries. Mortality rates are one of the indicators of poor health systems (Joubert et al., 2007). The most common medical complications were pressure sores, urinary tract infection and respiratory problems, faecal impaction, sepsis, deep vein thrombosis and psychological problems.

Frielingsdorf and Dunn (2007) found the highest mortality rate in South Africa, at 16\%, almost 10\% higher than any other South African study. This might be due to two reasons. Firstly the study participants consisted of patients with cervical injuries only. This cohort is at higher risk for early mortality (Krause, Carter, Pickelsimer and Wilson, 2008). Secondly the study was conducted in a tertiary hospital and not a spinal unit. Spinal cord injured patients, especially those with additional injuries or co-morbidities, are usually admitted to a general hospital and from there referred to a rehabilitation unit once medically stable. Thus mortality rates should be higher for hospitals than rehabilitation units.

Table 2.11 Length of stay, mortality rate and complications of SCIs in Africa

\begin{tabular}{|c|c|c|c|}
\hline Author(s) & Length of stay & $\begin{array}{l}\text { Mortality } \\
\text { rate }\end{array}$ & Complications \\
\hline $\begin{array}{l}\text { Iwegbu, 1983, } \\
\text { (Nigeria) }\end{array}$ & N/A & $25 \%$ & $\begin{array}{l}\text { Pressure sores } \\
\text { Urinary tract infections } \\
\text { Chest infections }\end{array}$ \\
\hline $\begin{array}{l}\text { Odendaal, 1991a, } \\
\text { (RSA: Gauteng } \\
\text { Province) }\end{array}$ & $\begin{array}{l}\text { Non Surgical } \\
\text { Mean: } 153 \text { days } \\
\text { Range: } 49-205 \text { days } \\
\text { Surgical } \\
\text { Mean: } 75 \text { days } \\
\text { Range: } 10.99 \text { to } 205\end{array}$ & $3 \%$ & Ureamia \\
\hline $\begin{array}{l}\text { Odendaal, 1991b, } \\
\text { (RSA: Gauteng } \\
\text { Province) }\end{array}$ & $\begin{array}{l}\text { Non-Surgical } \\
\text { Mean: } 105 \text { days } \\
\text { Range: } 69.3-139.3 \\
\text { days } \\
\text { Surgical } \\
\text { Mean: } 88.2 \text { days } \\
\text { Range: } 14.7-275 \text { days }\end{array}$ & N/A & $\begin{array}{l}\text { Urinary tract infection } \\
\text { Pressure sores } \\
\text { Psychological maladjustment } \\
\text { Sepsis }\end{array}$ \\
\hline $\begin{array}{l}\text { Couto \& Levy, } \\
\text { 1994, (Zimbabwe) }\end{array}$ & N/A & $50 \%$ & Respiratory problems \\
\hline
\end{tabular}




\begin{tabular}{|c|c|c|c|}
\hline $\begin{array}{l}\text { Hart \& Williams, } \\
\text { 1994, (RSA: } \\
\text { Gauteng Province) }\end{array}$ & 3-6 Months & $7 \%$ & $\begin{array}{l}\text { Pressures Sores } \\
\text { Severe spasticity } \\
\text { Debilitating pain } \\
\text { Urological complications }\end{array}$ \\
\hline $\begin{array}{l}\text { Velmahos et al., } \\
\text { 1995, (RSA: } \\
\text { Gauteng Province) }\end{array}$ & Not Available & $3 \%$ & $\begin{array}{l}\text { Sepsis } \\
\text { Pressure sores } \\
\text { Urinary tract infections } \\
\text { Deep vein thrombosis }\end{array}$ \\
\hline $\begin{array}{l}\text { Levy et al., 1998, } \\
\text { (Zimbabwe) }\end{array}$ & N/A & $7 \%$ & $\begin{array}{l}\text { Pressure sores } \\
\text { Urinary tract infections } \\
\text { Sepsis } \\
\text { Calcification } \\
\text { Muscle spasms } \\
\text { Contractures }\end{array}$ \\
\hline $\begin{array}{l}\text { Igun et al., 1999, } \\
\text { (Nigeria) }\end{array}$ & N/A & $26 \%$ & $\begin{array}{l}\text { Broncho-pneumonia } \\
\text { Urinary tract infection } \\
\text { Pressure sores }\end{array}$ \\
\hline $\begin{array}{l}\text { Solagberu, 2002, } \\
\text { (Nigeria) }\end{array}$ & $\begin{array}{l}\text { Mean :85 } \\
\text { Range :1-530 }\end{array}$ & $26 \%$ & $\begin{array}{l}\text { Bladder distension } \\
\text { Paralytic ileus } \\
\text { Faecal impaction } \\
\text { Urinary tract infection } \\
\text { Hypotension } \\
\text { Pressure sores } \\
\text { Hyperpyrexia } \\
\text { Depression } \\
\text { Deep vein thrombosis }\end{array}$ \\
\hline $\begin{array}{l}\text { Le Roux \& Dunn, } \\
2005 \text { (RSA, } \\
\text { Western Cape) }\end{array}$ & $\begin{array}{l}\text { Mean :30 } \\
\text { Range :4-109 }\end{array}$ & $2 \%$ & $\begin{array}{l}\text { Sepsis } \\
\text { Pneumonia } \\
\text { Pressure sores }\end{array}$ \\
\hline $\begin{array}{l}\text { Olasode et al., } \\
\text { 2006, (Nigeria) }\end{array}$ & N/A & $7 \%$ & Non-mentioned \\
\hline $\begin{array}{l}\text { Frielingsdorf \& } \\
\text { Dunn, 2007, (RSA, } \\
\text { Western Cape) }\end{array}$ & $\begin{array}{l}\text { Mean :32.7 } \\
\text { Range : } 2-454\end{array}$ & $16 \%$ & $\begin{array}{l}\text { Pressure sores } \\
\text { Atelectasis } \\
\text { Pneumonia }\end{array}$ \\
\hline $\begin{array}{l}\text { Udosen et al., } \\
\text { 2007, (Nigeria) }\end{array}$ & N/A & $14 \%$ & $\begin{array}{l}\text { Pressure sores } \\
\text { Paralytic ileus } \\
\text { Hyperpyrexia } \\
\text { Urinary tract infections } \\
\text { Respiratory difficulties }\end{array}$ \\
\hline $\begin{array}{l}\text { Emujulu et al., } \\
\text { 2009, (Nigeria) }\end{array}$ & N/A & $21 \%$ & Pressure sores \\
\hline $\begin{array}{l}\text { Obalum et al., } \\
\text { 2009, (Nigeria) }\end{array}$ & N/A & $18 \%$ & $\begin{array}{l}\text { Pressure sores } \\
\text { Urinary tract infections } \\
\text { Faecal impaction } \\
\text { Respiratory failure }\end{array}$ \\
\hline $\begin{array}{l}\text { Gosselin \& } \\
\text { Coppotelli, 2010, } \\
\text { (Sierra Leone) }\end{array}$ & $\begin{array}{l}\text { Mean :75 } \\
\text { Range :33-103 }\end{array}$ & $29 \%$ & No complications \\
\hline
\end{tabular}




\subsubsection{Epidemiology of spinal cord injuries in developed countries}

Studies published in the last two decades, from Australia, Canada, Greece, Iran, Ireland, Italy, Sweden, and the USA on epidemiology of spinal injuries will be reviewed. Results on demographics, causes, classification of injuries, LOS, mortality rate and complications will be explored.

Table 2.12 (next page) summarises the number of participants, study design, male-to-female ratio and the age of participants in studies published in the countries mentioned above. The majority of these studies were retrospective analysis of hospital records, established surveillance data or established national spinal cord registers. Australia was the first country to establish a national register for all SCIs (O'Connor, 2001). The USA has established surveillance of SCIs in various states. These surveillance or registers cover the majority of centres that manage SCIs in both countries. The duration of the retrospective analyses ranged over a period of 2-30 years. Studies from European countries reported on prospective studies using multi centre data. These studies lasted between one and two years resulting in fewer participants (O’Connor, 2006a; Pagliacci, Franceschini, Di Clemente, Agosti and Spizzichino, 2003; Divanoglou and Levy, 2009).

The mean age ranged between 29 and 50, with Canada having the highest mean age (50) (Kattail, Furlan and Fehlings, 2009) and USA the lowest (29) (Thurman, Burnett, Beaudoin, Jeppson and Sniezek, 1995). The difference in the mean age could be attributed to the different demographics of the various regions in both countries. Canada had the widest age range, from 9-102 (Pickett, CamposBenitez, Keller and Duggal, 2006; Kattail et al., 2009). The male-to-female ratio was higher in European countries compared to USA, Canada and Australia.

Retrospective studies using data from surveillance databases or national registers is useful in epidemiological studies as they yield more organised data and it is easy to obtain a large number of participants and cover a large population area (Jackson, Dijkers, DeVivo, Pokzatek, 2004; Nobunaga, Go and Karunas, 1999). 
Table 2.12: Age and gender analysis on SCIs in developed countries

\begin{tabular}{|c|c|c|c|c|c|c|}
\hline Author (s) & $\begin{array}{l}\text { No. of } \\
\text { partici- } \\
\text { pants }\end{array}$ & $\begin{array}{l}\text { Male-to- } \\
\text { female } \\
\text { ratio }\end{array}$ & $\begin{array}{l}\text { Mean } \\
\text { age }\end{array}$ & $\begin{array}{l}\text { Most } \\
\text { affected } \\
\text { age ranges }\end{array}$ & $\begin{array}{l}\text { Type of study } \\
\text { and data } \\
\text { source }\end{array}$ & $\begin{array}{l}\text { Study } \\
\text { period }\end{array}$ \\
\hline $\begin{array}{l}\text { Thurman et al., } \\
\text { 1995, (USA) }\end{array}$ & 223 & $3: 1$ & $\begin{array}{l}29 \\
\text { (median) }\end{array}$ & $15-24$ & $\begin{array}{l}\text { Multi-centre } \\
\text { (Hospitals ) in } \\
\text { Utah }\end{array}$ & 3 years \\
\hline $\begin{array}{l}\text { Nobunaga et } \\
\text { al., 1999, } \\
\text { (USA) }\end{array}$ & 25,054 & $4.4: 1$ & 32.3 & $\mathrm{NM}$ & $\begin{array}{l}\text { Retrospective; } \\
\text { National } \\
\text { Register of SCI }\end{array}$ & 25 years \\
\hline $\begin{array}{l}\text { Sekhon and } \\
\text { Fehlings, } 2001 \\
\text { (USA) }\end{array}$ & NM & 3 to $4: 1$ & NM & $21-30$ & $\begin{array}{l}\text { A Review of } \\
\text { studies }\end{array}$ & $\mathrm{NM}$ \\
\hline $\begin{array}{l}\text { O’Connor, } \\
2002 \mathrm{a}, \\
\text { (Australia) }\end{array}$ & 265 & $3.2: 1$ & NM & $15-24$ & $\begin{array}{l}\text { Retrospective; } \\
\text { National } \\
\text { Database }\end{array}$ & 2 years \\
\hline $\begin{array}{l}\text { O'Connor, } \\
2002 \mathrm{~b}, \\
\text { (Australia) }\end{array}$ & $\begin{array}{l}57 \\
\text { MVA } \\
\text { related in } \\
\text { South } \\
\text { Australia }\end{array}$ & 2.9:1 & & $15-24$ & $\begin{array}{l}\text { Retrospective; } \\
\text { National } \\
\text { Database }\end{array}$ & 8 years \\
\hline $\begin{array}{l}\text { Pagliacci et al., } \\
\text { 2003, (Italy) }\end{array}$ & 684 & $4: 1$ & $\mathrm{NM}$ & $25-44$ & $\begin{array}{l}\text { Prospective; } \\
\text { Multi-centre ( } 32 \\
\text { Hospitals) }\end{array}$ & $\begin{array}{l}1 \text { year, } 11 \\
\text { months }\end{array}$ \\
\hline $\begin{array}{l}\text { Pickett et al., } \\
\text { 2003, Canada }\end{array}$ & 385 & $\begin{array}{l}<10 \text { and } \\
70+: 1.5: 1 \\
10-69: 2.2 \\
\text { to } 3.5: 1\end{array}$ & NM & NM & $\begin{array}{l}\text { Retrospective; } \\
\text { Hospital based }\end{array}$ & 5 years \\
\hline $\begin{array}{l}\text { Jackson et al., } \\
\text { 2004, (USA) }\end{array}$ & 30,532 & $4: 1$ & 33 & $16-30$ & $\begin{array}{l}\text { Retrospective ; } \\
\text { National } \\
\text { Register of SCI. }\end{array}$ & 30 years \\
\hline $\begin{array}{l}\text { O’Connor \& } \\
\text { Murray, 2006, } \\
\text { (Ireland) }\end{array}$ & 46 & $6.7: 1$ & $\begin{array}{l}37 \\
\text { (median) }\end{array}$ & $20-30$ & $\begin{array}{l}\text { Prospective; } \\
\text { National SCI } \\
\text { centre }\end{array}$ & 1 year \\
\hline $\begin{array}{l}\text { O'Connor and } \\
\text { Brown, 2006, } \\
\text { (Australia) }\end{array}$ & $\begin{array}{l}161 \\
\text { MVA } \\
\text { related in } \\
\text { Victoria }\end{array}$ & 2.3:1 & $\mathrm{NM}$ & $15-24$ & $\begin{array}{l}\text { Retrospective; } \\
\text { National } \\
\text { Database }\end{array}$ & 15 years \\
\hline $\begin{array}{l}\text { Pickett et al., } \\
\text { 2006, (Canada) }\end{array}$ & 151 & $3: 1$ & 42.2 & $20-29$ & $\begin{array}{l}\text { Retrospective; } \\
\text { Hospital based }\end{array}$ & $\begin{array}{l}4 \text { years, } 5 \\
\text { months }\end{array}$ \\
\hline $\begin{array}{l}\text { Divanoglou \& } \\
\text { Levy, 2009, } \\
\text { (Sweden, } \\
\text { Greece) }\end{array}$ & 128 & $\begin{array}{l}7.3: 1 \\
\text { (Sweden) } \\
3,4: 1 \\
\text { (Greece) }\end{array}$ & 45 & $16-45$ & $\begin{array}{l}\text { Prospective; } \\
\text { Multi-centre }\end{array}$ & 1 year \\
\hline $\begin{array}{l}\text { Kattail et al., } \\
\text { 2009, (Canada) }\end{array}$ & 569 & $2.3: 1$ & 50 & & $\begin{array}{l}\text { Retrospective; } \\
\text { Hospital and } \\
\text { National trauma } \\
\text { registry }\end{array}$ & 12 years \\
\hline
\end{tabular}

Note: Not mentioned (NM) 
According to Table 2.13 motor vehicle accidents (MVA) were the leading cause of SCIs in the majority of developed countries, followed by falls. However, in Canada and Sweden falls were the major cause of SCIs (Pickett, Simpson, Walker and Brison, 2003; Kattail et al., 2009; Divanoglou and Levy, 2009). These falls were more prevalent in the older population (Pickett et al., 2003). Work- and domestic-related SCIs accounted for between 4 and 21\% of SCI according to Table 2.13. Note that Iran is included in this and the following tables.

Table 2.13 Causes of SCIs in developed countries

\begin{tabular}{|c|c|c|c|c|c|}
\hline Author(s) & $\begin{array}{l}\text { Road } \\
\text { traffic } \\
\text { accidents }\end{array}$ & Falls & $\begin{array}{l}\text { Violence } \\
\text { (gunshot and } \\
\text { stabbing) }\end{array}$ & Sport & $\begin{array}{l}\text { Domestic or } \\
\text { occupational } \\
\text { related }\end{array}$ \\
\hline $\begin{array}{l}\text { Thurman et al., 1995, } \\
\text { (USA) }\end{array}$ & MVA only & & & & \\
\hline $\begin{array}{l}\text { Nobunaga et al., } \\
\text { 1999, (USA) }\end{array}$ & $43 \%$ & $19 \%$ & $19 \%$ & $11 \%$ & $8 \%$ \\
\hline $\begin{array}{l}\text { Sekhon and Fehlings, } \\
\text { 2001, (USA) }\end{array}$ & $40 \%-50 \%$ & $20 \%$ & $10 \%-25 \%$ & $\begin{array}{l}10 \%- \\
25 \%\end{array}$ & $10 \%-25 \%$ \\
\hline $\begin{array}{l}\text { O’Connor, 2002a, } \\
\text { (Australia) }\end{array}$ & $43 \%$ & $31 \%$ & $0 \%$ & $8 \%$ & $18 \%$ \\
\hline $\begin{array}{l}\text { O’Connor, 2002b, } \\
\text { (Australia) }\end{array}$ & MVA only & & & & \\
\hline $\begin{array}{l}\text { Pagliacci et al., 2003, } \\
\text { (Italy) }\end{array}$ & $43 \%$ & $19 \%$ & $19 \%$ & $8 \%$ & $11 \%$ \\
\hline $\begin{array}{l}\text { Pickett et al., 2003, } \\
\text { (Canada) }\end{array}$ & $43 \%$ & $43 \%$ & $2 \%$ & $2 \%$ & $10 \%$ \\
\hline $\begin{array}{l}\text { Jackson et al., 2004, } \\
\text { (USA) }\end{array}$ & $46 \%$ & $20 \%$ & $18 \%$ & $10 \%$ & $6 \%$ \\
\hline $\begin{array}{l}\text { O'Connor and } \\
\text { Murray, 2006, } \\
\text { (Ireland) }\end{array}$ & $50 \%$ & $37 \%$ & $0 \%$ & $9 \%$ & $4 \%$ \\
\hline $\begin{array}{l}\text { O’Connor and } \\
\text { Brown, 2006, } \\
\text { (Australia) }\end{array}$ & MVA only & & & & \\
\hline $\begin{array}{l}\text { Pickett et al., 2006, } \\
\text { (Canada) }\end{array}$ & $47 \%$ & $31 \%$ & $5 \%$ & $9 \%$ & $8 \%$ \\
\hline $\begin{array}{l}\text { Chabok et al., 2009, } \\
\text { (Iran) }\end{array}$ & $52 \%$ & $43 \%$ & $0 \%$ & $0 \%$ & $5 \%$ \\
\hline \multirow{2}{*}{$\begin{array}{l}\text { Divanoglou and } \\
\text { Levy, 2009, } \\
\text { (Sweden, Greece) }\end{array}$} & $23 \%$ & $47 \%$ & $1 \%$ & $8 \%$ & $21 \%$ \\
\hline & $41 \%$ & $37 \%$ & $2 \%$ & $3 \%$ & $17 \%$ \\
\hline $\begin{array}{l}\text { Kattail et al., 2009, } \\
\text { (Canada) }\end{array}$ & $26 \%$ & $50 \%$ & $2 \%$ & $14 \%$ & $8 \%$ \\
\hline $\begin{array}{l}\text { Rahimi-Movaghar et } \\
\text { al., 2009, (Iran) }\end{array}$ & $75 \%$ & $25 \%$ & 0 & 0 & 0 \\
\hline
\end{tabular}


Table 2.14 summarises the classification of SCIs in developed countries and Iran. The vast majority of the injuries were incomplete (ASIA B-E), the most common incomplete category being ASIA D (Jackson et al., 2004; Pickett et al., 2006; Kattail et al., 2009). All studies used the ASIA scale to determine the extent of the SCI, except one done in the USA which used the Frankel scale (Thurman et al., 1995). Four studies combined thoracic and lumbar injuries (paraplegia) (O'Connor, 2002b; Jackson et al., 2004; Pagliacci et al., 2003; Dianoglou and Levy, 2009). This makes it difficult to determine the most affected region of the spine, although it appears that, overall, the cervical region was the most affected region. A study done in Iran showed the lumbar region of the spine as being the most affected (Chabok, Safaee, Alizadeh, Dafchahi, Taghinnejadi and Koochakinejad, 2009).

Table 2.14: Classification of SCIs in developed countries

\begin{tabular}{|c|c|c|c|c|c|}
\hline Author(s) & Cervical & Thoracic & Lumbar & $\begin{array}{l}\text { ASIA A } \\
\text { (Complete) }\end{array}$ & $\begin{array}{l}\text { ASIA B-E } \\
\text { (Incomplete) }\end{array}$ \\
\hline $\begin{array}{l}\text { Thurman et al., 1995, } \\
\text { (USA) }\end{array}$ & $59 \%$ & $41 \%$ & $0 \%$ & $38 \%$ & $62 \%$ \\
\hline $\begin{array}{l}\text { Nobunaga et al., 1999, } \\
\text { (USA) }\end{array}$ & $54 \%$ & $35 \%$ & $11 \%$ & $49 \%$ & $51 \%$ \\
\hline $\begin{array}{l}\text { Sekhon and Fehlings, } \\
\text { 2001, (USA) }\end{array}$ & $50 \%$ & $30 \%$ & $20 \%$ & $45 \%$ & $55 \%$ \\
\hline $\begin{array}{l}\text { O’Connor, 2002a, } \\
\text { (Australia) }\end{array}$ & $58 \%$ & $25 \%$ & $17 \%$ & $37 \%$ & $63 \%$ \\
\hline $\begin{array}{l}\text { O'Connor, 2002b, } \\
\text { (Australia) }\end{array}$ & $35 \%$ & $65 \%$ & $0 \%$ & $35 \%$ & $65 \%$ \\
\hline $\begin{array}{l}\text { Pagliacci et al., 2003, } \\
\text { (Italy) }\end{array}$ & $40 \%$ & $60 \%$ & $0 \%$ & $51 \%$ & $49 \%$ \\
\hline $\begin{array}{l}\text { Jackson et al., } 2004 \text {, } \\
\text { (USA) }\end{array}$ & $54 \%$ & $46 \%$ & $0 \%$ & $44 \%$ & $56 \%$ \\
\hline $\begin{array}{l}\text { O'Connor and Murray, } \\
\text { 2006, (Ireland) }\end{array}$ & $50 \%$ & $41 \%$ & $9 \%$ & $39 \%$ & $61 \%$ \\
\hline $\begin{array}{l}\text { Pickett et al., 2006, } \\
\text { (Canada) }\end{array}$ & $75 \%$ & $10 \%$ & $15 \%$ & $46 \%$ & $54 \%$ \\
\hline $\begin{array}{l}\text { Chabok et al., } 2009 \text {, } \\
\text { (Iran) }\end{array}$ & $13 \%$ & $5 \%$ & $82 \%$ & $6 \%$ & $94 \%$ \\
\hline \multirow{2}{*}{$\begin{array}{l}\text { Divanoglou and Levy, } \\
\text { 2009, (Sweden and } \\
\text { Greece) }\end{array}$} & $45 \%$ & $55 \%$ & $0 \%$ & NA & NA \\
\hline & $48 \%$ & $52 \%$ & $0 \%$ & NA & NA \\
\hline $\begin{array}{l}\text { Kattail et al., 2009, } \\
\text { (Canada) }\end{array}$ & $61 \%$ & $11 \%$ & $28 \%$ & $12 \%$ & $88 \%$ \\
\hline $\begin{array}{l}\text { Rahimi-Movaghar et } \\
\text { al., 2009, (Iran) }\end{array}$ & $25 \%$ & $25 \%$ & $50 \%$ & $25 \%$ & $75 \%$ \\
\hline
\end{tabular}




\subsubsection{Length of stay, mortality rate and complications of spinal cord injuries}

Most studies reviewed lack information related to LOS and mortality rates as most were reporting on incidence and prevalence results. According to Pickett et al (2006) the average LOS was 16.8 days with a range of 1-186 days and an eight percent mortality rate. Kattail et al (2009) reported different results: 29.3 days mean LOS with a range of 1-114 days and a mortality rate of four percent. The low average LOS could be due to the fact that most participants in the study were classified as incomplete lesions. Thurman et al. (1995) reported a mortality rate of $20.9 \%$ in USA whereas Sekhon and Fehlings (2001) reported a mortality rate range of 4.4 to $16.7 \%$.

The most commonly cited medical complications were contractures, heterotrophic ossification, atelectasis/pneumonia, cardiac arrest, renal failure, pressures sores, pulmonary emboli, deep vein thrombosis, pain and sepsis (Pickett et al., 2006; Sekhon and Fehlings, 2001; Pagliacci et al., 2003; Rahimi-Movaghar, Saadat, Rasouli, Ganji, Ghahramani, Zarei and Vaccaro, 2009; McKinley et al., 1999).

\subsection{Rehabilitation Cost Of Spinal Cord Injuries}

SCI injuries exert an enormous burden on the already over-burdened healthcare system worldwide (Ackery et al., 2004). It is imperative to determine the costs of these devastating injuries to make sure that resource allocation is both equitable and meets the needs of people with SCIs. The most commonly used variables in studies determining the costs of SCIs are length of stay (acute hospital stay and in-patient rehabilitation) and cost of hospital stay per day (Dryden, Saunders, Jacobs, Schopflocher, Rowe, May, Yiannakoulias, Svenson and Voaklander, 2004; Fiedler, Laud, Maiman \&Apple, 1999; Cifu, Huang, Kolakowsy-Hayner and Seel, 1999a; Cifu, Seel, Kreutzer, Marwitz, McKinley and Wisor, 1999b).

These variables are then compared with other variables (such as the neurological level of the individuals, causes of SCI, functional abilities on admission and discharge, gender and age) to determine the impact of various variables on differences in cost. Although the majority of the studies reported on the overall costs of stay, some studies explored and itemised each service rendered and compared the costs of each in relation to cause, age, gender and neurological level of the injury or completeness of the injury (French, Campbell, Sabharwal, Nelson, Palacios, Gavin-Deschnack, 2007).

The results of these studies were difficult to compare as they differ in methodology, choice of variables measured and sources of data. However, older age, completeness of injury, tetraplegia, low 
FIM (motor part of the FIM+FAM) on admission were associated with higher LOS and therefore high costs (Cifu et al., 1999a, Cifu et al., 1999b). There are high costs associated with SCIs and every attempt should be made to prevent them. However, Saari (2001) believes that costs only should not be the driving force of prevention, but rather the quest to improve the quality of life.

\subsection{Prevention Of Spinal Cord Injuries In General}

There are three layers of injury prevention according to the principles of epidemiology namely primary, secondary and tertiary prevention (Joubert et al., 2007). Primary prevention seeks to totally eliminate the injury incident from occurring, secondary prevention minimises the severity of injuries that occurs and tertiary prevention involves efforts following the incident that will optimise the outcomes, regardless of severity of the injury i.e. rehabilitation. According to O'Hare and Hall (1997) environmental modification, change in legislation and education of persons at risk are the three major components of primary prevention. Rehabilitation professionals can play a major role in prevention of SCIs in society in general or in industries by providing health-promotion activities, including health education and ergonomics (Naidoo and Wills, 2009).

The first step towards prevention of SCIs is the development of a surveillance system (O'Connor, 2000; Horan and Mallonee, 2003). A surveillance system collects data that will enable policy makers and government to quantify the extent of SCIs in their country, so as to better allocate resources towards prevention. Developed countries like Australia, Canada and the USA are examples in this field. They have established national registries for SCIs and have standardised the information to be collected to make comparison of data easier both nationally and internationally (DeVivo, BieringSørensen, Noonan, Post, Stripling and Wing, 2006; O’Connor, 2000, Horan and Mallonee, 2003).

In Australia, prevention of SCIs was based on analysing the causal factors and epidemiological trends. Road traffic accidents, sports, falls and violence were amongst the causes cited. This was followed by a programme that was aimed at the different causal factors (Yeo, 1993). The first programme was aimed at primary and secondary school-going children to prevent swimming-related and road trafficrelated SCIs (by wearing seat belts and helmets) (Yeo 1987). The results of the study demonstrated the success of a programme in educating school-going children about prevention of SCIs. Another study done in Canada investigated the impact of a SCI preventative programme amongst teenagers. The study showed most schools used the programme especially those with swimming pools (Bhide et al., 2000). However, the study does not report on the reduction of incidence of SCIs in this cohort. According to Rosenberg, Zirkle and Neuwelt (2005) the Think First programmes aimed at preventing SCIs were successful in the USA. These programmes were youth orientated, reinforced public 
education programme and a programme that was designed to influence policy and attitude changes (Rosenberg et al., 2005).

According to Hardcastle (1987) SCIs should be recognised as a national health priority by any government due to the high costs associated with these injuries. He further suggested the establishment of a national register; that prevention should be encouraged through education and awareness campaigns and the enforcement of the current legislative framework or the introduction of new legal frameworks to combat the causes of SCIs in various sectors. Pless (2000) and Noakes (2007) emphasize the fact that surveillance should be strengthened in order to develop preventive strategies.

\subsection{Prevention Of Spinal Cord Injuries In The Workplace}

There is paucity in literature on prevention of SCIs in the workplace. However, there are studies that look at prevention of work-related injuries. According to Smith (2001), there are four public health approaches to injury prevention in the workplace: surveillance and risk factor identification, intervention development and evaluation, identification/development of avoidance/control strategies and practical approaches to implementation. He further identifies the design of engineering solutions, personal protective equipment and educational interventions as major components of the intervention programmes.

According to Higgins, Casini, Bost, Johnson and Rautiainen (2001), the Fatality Assessment and Control Evaluation (FACE) programme was developed in an attempt to prevent occupational related injuries in the USA. This programme was successful in determining factors that contributed to the injuries and the development of preventative strategies.

The task of preventing SCIs in the workplace is tremendous and the responsibility of both the employer and the employee. Labour legislation around the world, and in South Africa, clearly stipulates the responsibility of the employer and the employee in preventing and managing injuries in the workplace.

According to the OHSA, the employer should take reasonable measures to prevent work-related injuries from occurring in the workplace. It includes the promotion of health and safety measures in the workplace which should be adhered to by the employer and the employees. The greatest responsibility lies with the employer for enforcing the policy directives amongst the employees, as there are punitive measures in place for those employers who are not compliant. 
According to the Labour Relations Act of 1995 and the Basic Conditions of Employment Act of 1997, employees are contractually required to obey the health and safety rules or measures put in place by the employer. Employees are therefore obliged to take reasonable care of their own health and safety and to minimise the occurrence of occupational injuries.

However a study published by Chetty, Jelsma and Maart in 2009 revealed that the majority of the employees were not compliant with health and safety behaviours in a beverage company in South Africa. The activities observed during this study were good housekeeping, stacking and storage, walkways, fire protection, prevention and emergency response, safety devices and personal protective equipment (PPE), hand tools, work area, manual lifting techniques, incident reporting and general safety. The most common areas of non-compliance were following signs depicting danger, PPE not issued, items not stored correctly, tools not in good condition, poor ergonomics and incorrect lifting techniques. Another study reported similar results in terms of non-compliance of employees regarding health and safety in the workplace (Akbar-Khanzadeh, Bisesi and Rivas, 1995, Gershon, Vlahov, Felknor, Vesley, Johnson, Delcios and Murphy, 1995).

Akbar-Khanzadeh et al., (1995) investigated the comfort of PPE amongst employees in an automobile plant in the USA. A large number of employees expressed discomfort with wearing PPE. According to the authors, this discomfort could lead to more injuries as the employees' compliance in wearing PPE decreases. Gershon, Vlahov, Felknor, Vesley, Johnson, Delcios and Murphy (1995) investigating compliance of healthcare workers in three hospitals in the USA regarding universal precautions, showed lack of compliance in wearing of protective clothing and eye protection. The authors further explained that compliance was associated with perceived organisational devotion to protection of employees, risk -taking personality, insight of risk and guidance in universal precautions. In this study, this could relate to the use of a harness amongst construction workers to prevent falls when on a scaffold, that could subsequently result in a spinal cord injury.

According to Hu, Lee, Shiao and, Guo (1998) employers lack general awareness of occupational health and safety. This study was published in Taiwan more than a decade ago. The situation might have improved due to increased levels of law enforcement in various countries, including in South Africa. Weil (1991) emphasises that the labour unions have a vital and a positive role to play in enforcing of occupational health and safety. This motion is supported by Harcourt, Wood and Harcourt (2004).

Prevention of work-related injuries in an occupational setting requires an integrated approach. Furthermore it is essential to have the necessary information before a preventative programme is embarked upon. This study will attempt to provide information that will assist the employers, policy makers and government to draw up preventative strategies for the workplace. 


\subsection{Chapter Summary}

The chapter started with an explanation of the anatomy and physiology of the spinal cord. The anatomical orientation of the spinal cord and its functions in relation to transmission and controlling sensory and motor information between the brain and other structures of the body was explored. This was followed by an explanation of the patho-physiology following the injury of the spinal cord. The primary phase after injury refers to the physiological response following an injury. The secondary phase involves the vascular changes and ischemia that follows the primary phase which can lead to further damage to the cord. Medical and surgical interventions are aimed at alleviating the consequences of this phase. Spinal cord injuries are classified according to the level of injury on the cord (paraplegia or tetraplegia) and the completeness (complete or incomplete) of the injury (extent of neurological damage to the cord).

Next the role of rehabilitation and SCI was discussed. The fundamental aim of rehabilitation is to return the individual who sustained a SCI back to his environment. The delivery of rehabilitation programmes for SCI individuals is done using the multidisciplinary approach where there is close collaboration of the team in an attempt to achieve the goals of rehabilitation. The types of rehabilitation that are commonly rendered to individuals with SCI are medical rehabilitation, vocational rehabilitation, diet therapy, cognitive behavioural therapy and counselling. Outcomes of SCI rehabilitation can be measured through a variety of tools.

Literature relating to the epidemiology of work-related SCIs from developed countries was surveyed. The male-to-female ratio ranges between 11.5:1 and 24:1. Falls were the major cause of SCIs at work followed by road traffic accidents. The majority of the injuries were classified as incomplete. The industries most commonly affected were construction, service, farming, industrial, transportation, and logging. The mortality rate reported was $8 \%$ and there was no information on rehabilitation and the LOS and secondary complications.

In Africa, studies on the epidemiology of SCI were retrospective and based on data obtained from hospital records. The age range of the participants was 15-40. Road traffic accidents, falls and violence are the most common causes of SCIs in Africa. The mean LOS ranged from 30-152 days and the actual range $2-530$. Mortality reported ranged between 1.9 to $50 \%$.

The epidemiology of SCIs in developed countries was slightly different from African countries. Most African studies were retrospective and used data from established institution- or national-based surveillance systems. The mean age ranged between 29 and 50. The male-to-female ratio ranged from 4 to 7.3:1. The most common causes were road traffic accidents and falls, which were similar to the findings on work-related SCIs. The majority of the injuries were classified as tetraplegia and 
incomplete. The mean LOS was 16.8 days and the range was from 1-186 days. The mortality rate reported was $8 \%$ which was similar to studies on work-related SCIs in Canada.

High costs of rehabilitation were recorded in developed countries. However, due to inconsistency in the techniques of data collection and presentation it was difficult to compare. High costs were associated with older age at time of injury, completeness of injury, tetraplegia and low FIM scores on admission.

Attempts to prevent SCIs, especially at work, should be directed at establishing the causes and other factors that are associated with these injuries. Prevention of work-related SCIs is the duty of the employer and the employee, as stipulated in the occupational health and safety regulations in South Africa.

The next chapter describes the methods used to collect and analyse the data in this study. 


\section{CHAPTER 3: RESEARCH METHODOLOGY}

\subsection{Introduction}

This chapter outlines the approaches used in this study to gather the information necessary to achieve the aims and objectives of the study. The setting of the study is described to contextualise the findings. The study population is explained as well as the inclusion and exclusion criteria used to select participants. Sampling methods and the sample size are explained, followed by the description of the tools used to gather the data. The pilot study and its findings are explained together with the recommendations. This is followed by the description of the procedures followed to gather and analyse the data. Ethical issues considered in this study conclude this chapter.

\subsection{Study Design}

This is a descriptive study in which mixed methods were utilised. Descriptive studies seek to quantify the extent of the problem and to describe extensively the current state of the situation of a particular aspect of health (Joubert et al., 2007). According to Domholdt (2005), Kroll, Neri and Miller (2005) and Carpenter and Suto (2008) mixed methods research includes the gathering and analysis of both quantitative and qualitative data in a single research study. Data can be collected concurrently or sequentially and merged at one or more stages during the research process.

For this study, a sequential approach was adopted where a dominant quantitative component was followed by a qualitative component. It is therefore necessary to explore the properties of both approaches and the theories that govern the integration of these methods. This will be followed by a description of the mixed method.

\subsubsection{Quantitative approach}

According to Domholdt (2005) the quantitative paradigm is characterised by its emphasis on measurement. It is also based on the notion that one can only be certain of knowledge which is verifiable through measurement and observation. This paradigm is based on the following assumptions (Domholdt, 2005):

1. Quantitative research determines the nature of reality through measurement and observation of the area of interest;

2. Quantitative research projects or controls reality;

3. The researcher should be independent of the subject or object investigated; 
4. Quantitative research yields information or results that can be generalised in different contexts;

5. In quantitative research, causes and effects can be determined and differentiated from each other and

6. In quantitative research, investigation or measurement is seen as an objective discovery of the truth and the researcher as an independent or impartial founder of the truths (Domholdt 2005).

Quantitative research therefore attempts to describe a phenomenon by measuring the extent of the problem and quantifying it with numbers. This study will use this approach to describe the epidemiology of work-related spinal cord injuries (SCI) in the study setting. This will be achieved by adopting the epidemiological triangle (Figure 3.1) which guides the researcher in obtaining information to identify the major risk factor categories for the disease or injury (Joubert et al., 2007). In this study the epidemiological triangle was utilised to gather information about the injured person (Host), the cause of the injury (Agent or Energy) and the physical or social environment (Context) in which these injuries occur.

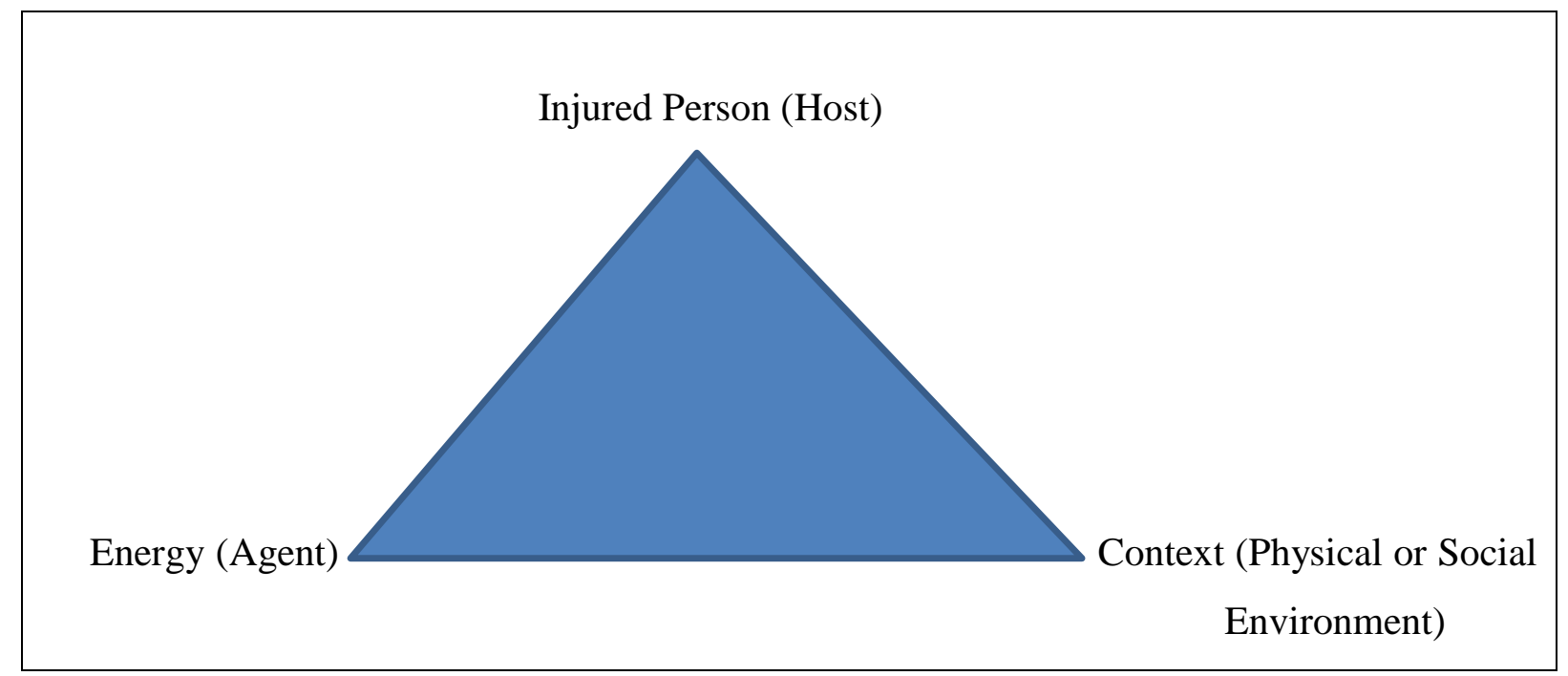

Figure 3.1: Epidemiological triangle (Adapted from Joubert et al., 2007) 


\subsubsection{Qualitative approach}

Carpenter and Suto, (2008:3) define qualitative research as "a situated activity that locates the observer in the world. It consists of a set of interpretive, material practices that make the world (the taken for granted in everyday life) visible (to others). It involves the studied use and collection of various types of empirical information using a diversity of methods, for example case study, personal narrative, artefacts, cultural texts, interviews, observations and visual records that describe routine and problematic moments in individuals' lives with the aim of developing a better understanding of the subject or phenomenon. Qualitative research privileges no single methodological practice over another and has no theory or paradigm that is distinctly its own."

According to Schwandt (2001), qualitative research aims at understanding human actions. It may involve both explicit and implicit comparisons and structured open-ended interviews. Schwandt (2001) further explains that qualitative means quality, which is inherent in the phenomena of something, which may be an object or experience. According to Domholdt (2005), there are five assumptions to better explain qualitative research.

1. The first assumption is that the world is constructed in multiple realities, meaning there are different meanings attached to events in our daily lives;

2. The second assumption explains the interdependent nature of the relationship between the investigator and the informant;

3. The third assumption is that knowledge is time and context-dependent. Knowledge obtained in a particular context is unique to that environment;

4. The fourth assumption is that it is impossible to establish a relationship between a cause and effect. It is more useful to describe and interpret events than to find a cause and an effect and

5. The fifth assumption is that investigation is value-bound and that research is influenced by the values of the researchers or the sources that fund it.

The researcher employed a qualitative research design when exploring the attitudes and knowledge of employees and employers regarding safety and health issues in the workplace. This method was used in an attempt to understand the meaning of events and interactions between employees and employers in a particular industry and their behaviour regarding compliance with health and safety regulations in their workplace. Hence, a descriptive phenomenological approach was used to collect key information from the informants. According to Carpenter and Suto (2008), descriptive phenomenology aims to create a detailed description of the specific experiences of others and ensures that the intrinsic meaning of the narratives is faithfully presented. The information gathered is context-specific and no attempts are made to establish a cause-effect relationship. 


\subsubsection{Mixed method approach}

One of the major strengths of mixed methods is that in a two-phased sequential design, Phase 1 results can be used to develop and inform the purpose and design of the subsequent Phase 2 components (Kroll et al., 2005). In this study, the results of Phase 1 determined the research settings (industries mostly affected by work-related SCIs) where key informants were identified, as well as the selection of key informants.

The different types of mixed methods are divided, based on two major categories: time order (concurrent versus sequential) and paradigm emphasis (equal status versus dominant status) (Kroll et al., 2005). Table 3.1 explains this phenomenon.

Table 3.1: Paradigms in mixed methods

\begin{tabular}{|l|l|l|l|l|}
\hline & Concurrent & Sequential & $\begin{array}{l}\text { Time order } \\
\text { Current study } \\
\text { design }\end{array}$ \\
\hline $\begin{array}{l}\text { Paradigm } \\
\text { Emphasis }\end{array}$ & Equal Status & QUAL+QUAN & $\begin{array}{l}\text { QUAL to } \\
\text { QUAN } \\
\text { QUAN to } \\
\text { QUAL }\end{array}$ & \\
\hline & $\begin{array}{l}\text { Dominant } \\
\text { Status }\end{array}$ & $\begin{array}{l}\text { QUAL + quan } \\
\text { QUAN +qual }\end{array}$ & $\begin{array}{l}\text { QUAL to quan } \\
\text { qual to QUAN } \\
\text { QUAN to qual } \\
\text { Quan to QUAL }\end{array}$ & QUAN to qual \\
\hline & & & \\
\hline
\end{tabular}

In this study, a sequential approach was adopted to collect information. The quantitative study was dominant as this phase attempted to answer the majority of the study objectives. The first phase comprised the quantitative component during which information from hospital records was collected to develop a profile of work-related SCI with regard to incidence, causes and mechanisms, the distribution of injuries according to industries and type of employment, the cost of rehabilitation and rehabilitation outcomes. During this phase the industries most commonly affected were identified; the qualitative phase of the study would be conducted in these industries.

The qualitative phase focused on aspects like the knowledge of employees and employers on safety and injury prevention, the compliance of the identified industries with the Occupational Health and Safety Act, (Act 85 of 1993, OSHA) and identifying existing preventative measures in these industries. The rationale behind the choice of design is that the complementary strengths of the two 
methods would enhance the findings of the study. Determining the magnitude of work-related SCIs (phase 1) would be of limited value if there was no in-depth investigation (phase 2) into the factors that led to employees sustaining these devastating injuries, and into possible preventative strategies.

\subsection{Research Setting}

The research was performed in two private SCI rehabilitation units in Gauteng, one of South Africa's nine provinces. Gauteng was selected as a study setting for many reasons: it is the smallest province in size, comprsing only $1.4 \%$ of the land area. It is highly urbanised (populated) with an estimated population of 10,451,713 (Community Survey, 2007). This translates into an average of 576 persons per $\mathrm{km}^{2}$. (According to Kok and Wentzel (2004) the only other province in South Africa with a population of more than 100 per $\mathrm{km}^{2}$ is KwaZulu-Natal with 105 persons per $\mathrm{km}^{2}$.). Table 3.2 shows the population growth of Gauteng Province since 1996. Between 1996-2001 the population grew by $20.4 \%$ and between $2001-2007$ by $13.9 \%$. This was second only to the Western Cape province whose population grew by $16.7 \%$. Migration of people from other countries and provinces accounts for $30 \%$ of the growth in Gauteng (Kok and Wentzel, 2004).

Table 3.2: Population estimates of Gauteng Province from 1996 to 2007

(Stats SA, 2007)

\begin{tabular}{|l|r|r|c|}
\hline Year & 1996 & 2001 & 2007 \\
\hline Population & 7624893 & 9178873 & 10451713 \\
\hline
\end{tabular}

Gauteng is also the centre of the South African economy, with a GDP for the region of more than R200bn since 1999. Between 2000-2001 Gauteng's GDP grew by more than three percent per annum. The unemployment rate in Gauteng was estimated to be $27 \%$ in 2001, which was lower than the national rate and the lowest when compared with the other provinces. This had risen to $31.8 \%$ in 2007 (now higher than in the Western Cape) but still lower than the South African national rate of 39\% (Kok and Wentzel, 2004).

In 2007 the average annual household income was R130,000, the highest in South Africa and is, with the Western Cape, one of the only two provinces to have an average of above R100,000. This is almost twice the South African average of R79,000. The majority of Gauteng's population has access to most of their basic needs i.e. water and sanitation, housing, electricity and telephones (Kok and Wentzel, 2004). 
Thus, with only $1.4 \%$ of South Africa's land area, the small province of Gauteng contributes $33 \%$ to the national economy and a phenomenal $10 \%$ to the GDP of the entire African continent, which makes it a leader in Africa. There are various types of industries that form the economy of Gauteng, ranging from a high-tech manufacturing and industrial sector and growing services and construction sectors to a thriving informal sector (Human Science Research Council, 2001).

The original study proposal called for a review of Department of Labour (Compensation Fund) records to identify all persons who suffered a work-related SCI during the study period. However, the Department of Labour refused access to these records for the purposes of the study and an alternative source of data needed to be found. This necessitated certain changes to the study objectives. For instance one of the major objectives was to identify the incidence of work-related SCI in Gauteng which was now no longer possible since the researcher had no way of ensuring he could identify the entire population. In addition the objective related to cost of SCI management could only be addressed in part since the only cost that could be determined was the cost of stay in rehabilitation units. The cost of acute care and assistive devices amongst others could not be determined from the records used.

Since most employees who get injured at work are admitted to private facilities, as the Department of Labour pays their medical bills (as provided for by the Compensation of Occupational Injuries and Diseases Act, 130 of 1993 and the Mine Health Safety Act 29 of 1996) the researcher then contacted the four private rehabilitation units in Gauteng that provide SCI rehabilitation. Of these, two (Life Riverfelt Lodge and Life Kensington) gave the researcher permission to peruse patient folders. Thus these two units were utilised as study settings.

Life Riverfelt Lodge was opened in September 2000. It is located north of Johannesburg in close proximity to the Life Fourways Hospital. It caters for all patients with neurological conditions. It has a capacity of 41 beds; the units are equipped with a dining room, visiting area and gymnasium for therapeutic activities, and offer patients access to a volley ball court. There are single- or two-bed rooms with en-suite bathrooms allowing patients to have acceptable levels of privacy.

Life Kensington was opened in July 1999. It has 52 beds (42 for adults and 10 for children). This facility is located in Kensington, east of Johannesburg. The rehabilitation unit houses dedicated adult and paediatric facilities, with large gyms and exterior therapy areas. The unit has two independent living units, as well as a purpose-built exterior wheelchair mobility training area. The facility caters for children as well with a dedicated paediatric team (Life Health Care, n.d.). 
The rehabilitation team in both facilities consisted of a team of physiotherapists and physiotherapy assistants; occupational therapists and occupational therapy technicians, speech therapists, a clinical psychologist, a dietician, medical doctors and nursing staff.

The researcher observed (in both study sites) during the data collection phase that on admission of the patient all members of the team assessed the patient individually and met to discuss their findings and formulate therapeutic management goals. They met once a week to discuss the progress of each discipline as well as how contextual factors might facilitate or act as barriers to rehabilitation. The researcher also noted a similar pattern of collaboration between professions as described by Sinclair et al. (2009). The occupational therapists (OTs) and physiotherapists (PTs) worked very closely with each other, which fostered a care philosophy. The dietician worked more closely with the nursing staff and the social worker with the psychologist.

\subsection{Study Population}

\subsubsection{Phase 1}

All persons who suffered work-related SCIs in Gauteng Province, South Africa, in the period, between 01 July 1999 (opening date of the first of the units) and 31 December 2009 (six months before data collection commenced) and who were admitted at either of the two study units formed the study population for Phase 1 of the study. The twelve year period chosen corresponds with the date of establishment of the research facilities as well as the ability of the information gathered to yield meaning results.

\subsubsection{Phase 2}

Employers and employees from the industries identified during phase 1 as being high risk for suffering work-related SCIs, and employees who had suffered a work-related SCI formed the study population for Phase 2. These industries were services, mining, engineering and construction.

\subsection{Inclusion And Exclusion Criteria}

\subsubsection{Phase 1}

\section{Inclusion criteria}

- Injury to the spinal cord resulting in permanent neurological damage, using the ASIA scale; 
- The person should have sustained the injury in the work environment and/or where the activity of the person at the time of injury was specified as working for earnings and

- Individuals eligible for full-time employment i.e. between 15-65-years-of-age at the time of admission with a SCI.

\section{Exclusion criteria}

- Injury to the spine that had completely resolved neurologically before discharge from the hospital;

- Files with so much missing data that it would have impacted negatively on the study findings;

- Since in South Africa any individual under the age of 15 years may not be engaged in full-time work, and the retirement age for workers is generally 65 years and above, any person who fell outside this age range was not considered for participation in this study

\subsubsection{Phase 2}

\section{Inclusion criteria}

- Full-time or permanently employed employees, including seasonal workers, in the identified industries who were employed at the time of data collection. By definition this included the following:

○ Managers or supervisors who were responsible for operations in a particular company;

○ Union members and shop stewards;

○ Employees who were health and safety representatives (designated according to the section 17 (1) of the OHSA, 1993);

○ Occupational hygienists and SHE coordinators and

○ Employees who had sustained a work-related SCI during the study period.

\section{Exclusion criteria}

- Employees with cognitive or speech impairments were excluded from the study as it would have been difficult to conduct an interview with them. 


\subsection{Sampling Methods And Sample Size}

\subsubsection{Phase 1}

All files of employees with SCIs, who had been admitted to the two Life Health Care Rehabilitation Units, were retrieved. A total of 140 files were identified. Five files had to be excluded due to missing clinical data. No further sampling was done. Thus a total of 135 files were perused to obtain data for the first phase of the study.

\subsubsection{Phase 2}

Phase 2 of data collection could not take place as originally proposed. The proposal called for identification of industries where work-related SCI occurred most from the results of Phase 1 of this study. One company per industry type would have been selected through purposive sampling for inclusion in the study.

Study participants from those companies would be identified through purposive sampling. Purposive sampling was to be used to handpick participants who would be able to supply the information necessary to answer the study's aims and objectives (Domholt, 2005). According to Joubert et al., 2007, purposive sampling is where the researcher makes sure that the chosen respondents or settings cover the full range of possible characteristics. In this study, employees, SHE coordinators, managers or supervisors, shop stewards, union members and those individuals who had sustained spinal cord injuries in the workplace, would have been purposefully selected to ensure they could provide the information to answer objectives 7-9 of the study. Interviews would have been performed until data saturation occurred.

However, the researcher was refused permission to perform the study by all the companies that were identified and approached, viz. Stallion Security, South African Police Service, Anglo-Gold Mines SA and PPC Cement. Thus the researcher could only interview participants who had sustained workrelated SCIs in the workplace in Phase 2 of the study. They were identified through their rehabilitation records at the research sites. Five were sampled purposively. The researcher sampled these five persons from the industries most affected by SCI to make sure that information yielded from the interviews encompasses different types of industries. While small samples are deemed adequate in qualitative investigation since the depth of information gathered from participants is more important than the actual number of participants the researcher acknowledges that five participants might have been to few. A further limitation of this phase was that information gathered lacked sufficient depth and that the researcher did not try to offset this by going back to participants to further explore issues or by interviewing additional participants. 


\subsection{Data Collection Tools}

\subsubsection{Phase 1}

A data coding form was developed to collect data from the two Life Health Care Rehabilitation units' files on employees who suffered spinal cord injuries (Appendix A). This form was developed by the researcher in consultation with experts in the field as well as a statistician. Section A of the tool gathered information related to demography e.g. gender, age and occupational-related information. Section B contained information related to the injury including the neurological level, the classification of the injury according to the ASIA scale, time of injury and information on the duration of stay in the rehabilitation facility. Section $\mathrm{C}$ gathered information on costs of medical rehabilitation and the outcomes of rehabilitation, using the FIM and FAM scores on admission and discharge. (The FIM+FAM is the outcome measurement tool used by the rehabilitation units to chart the progress of in-patients.)

The FIM and FAM (Functional Independence Measure and Functional Assessment Measure) were developed to assess the level of motor activity and psychological functioning of a patient with a neurological insult (Turner-Stokes, Nyein, Turner-Stokes and Gatehouse, 1999). The motor functioning aspects include eating, swallowing, grooming, bathing, dressing upper and lower body, toileting, bladder and bowel function, various transfers, locomotion and stairs and community mobility. A total motor sub score of between 16 and 112 can be achieved. The psychological (cognitive) functioning aspect includes comprehension, expression, reading, writing, speech intelligibility, social interaction, emotional status, adjustment to limitations, leisure activities, problem solving, memory, orientation, concentration and safety awareness. A total cognitive sub score of between 14 and 98 can be achieved. Scoring of the patient on the FIM and FAM is done through clinician observation of these activities. Assessment is done by members of the multidisciplinary team. The scoring depends on the level of assistance that a patient requires to perform an activity. The scoring ranges from 1-7, where 1 is total assistance (complete dependence) and 7 is complete independence (Turner-Stokes et al., 1999). The tool has been found to be valid and reliable (McPherson, Pentland, Cudmore and Prescott, 1996; Hawley, Taylor, Hellawell and Pentland, 1999; Skinner and Turner-Stokes, 2006).

This tool was used in both rehabilitation facilities to assess the level of functioning of patients on admission and on a weekly basis through their rehabilitation stay. The clinicians in the two facilities received training on the scoring of the tool which improves reliability. The researcher used scores as determined by the clinicians on admission and on discharge to rehabilitation to evaluate the total effect of the rehabilitation interventions on clients' cognitive and physical function. 
Section D gathered information on the outcomes of rehabilitation using the participation component of the ICF checklist on selected ICF domains (Kirchberger et al., 2010. The following domains were selected: communication, self-care, mobility, domestic life, community, social and civic life and employment. These domains were selected because they are most frequently impacted on by SCI (Kirchberger et al., 2009). The ICF checklist and the FIM and FAM are validated tools in the measurement of status of disability amongst people with SCIs. Data gathered through these forms were analysed to answer objectives 1-7 of the study. Section D of Appendix A aimed at collecting post-discharge information. However, there was not enough data to fully gather the required information from the files and analysis of this data was not included in this study.

The researcher used the combination of the three above-mentioned outcomes measurement tools to assess the level of disability at various levels of injury. The ASIA scale focuses on assessing impairments; the FIM+FAM focuses on assessing the activity limitations of an individual with SCI while the ICF check list focuses on assessing participation limitations. A combination of these tools gives rehabilitation professionals an overall understanding of the impact of the SCI on the individual to enable them to plan an appropriate and specific rehabilitation intervention.

\subsubsection{Phase 2}

An interview schedule (Appendix B) was developed by the researcher to use for guidance when conducting unstructured interviews with line managers, supervisors, SHE representatives, occupational hygienists and SHE coordinators in various companies. These interviews were to determine the attitudes, level of knowledge and compliance in the company with the OSHA's safety rules and regulations.

Another interview schedule (Appendix C) was developed by the researcher to use as guidance when conducting unstructured interviews with employees and union representatives to determine their level of knowledge of the OSHA's rules and regulations in the workplace and their opinion on the company's adherence to these.

The researcher could not use either of these schedules as he was refused permission to access the targeted participants in various companies.

Appendix D, an interview schedule, was used to gather relevant information from those employees who had sustained work-related SCIs. All interview schedules had a section for demographic information of participants, e.g. their position in the company, age, gender etc. This was the only appendix that was successfully used by the researcher to gather relevant information from the targeted 
participants. Information gathered from this sample yielded the employee's (i.e. the person who sustained a SCI at work) perspective only, since efforts to reach their colleagues were unsuccessful.

\subsection{Pilot Study}

A pilot study was conducted to test the methodology of the study and, more especially, the feasibility of the measuring instruments.

\subsubsection{Phase 1}

The data coding form (Appendix A) was completed with five folders obtained from Entabeni Life Rehabilitation in Durban. The folders were not part of the main study. During this process the following issues were assessed:

- Whether the information in the folders was sufficient to answer the questions on the data coding form or if some questions should be deleted. In the demographic information, Section A, level of education was omitted as it proved difficult to get this information from the file.

- If the ICF-based participation measure could be completed through the information in the folder; or, if another participation measure was used during rehabilitation, whether this would be more advantageous to use in the study and possibly replace the ICF measure in the data coding form. No other participation measure was used by the study centres and the information was sufficient to code the data onto the ICF tool.

- Whether the questions should follow more or less the same sequence as data in the folders or if a different sequence might ease data collection.

- Whether the data collected would provide the necessary information to answer the aim and objectives of the study relevant to this data. This was done in consultation with a statistician.

- How long it took to complete the data coding form, in order to assist planning the data collection phase. It took approximately an hour for the researcher to extract all relevant information from the files. This information assisted the researcher to estimate the time it would take to collect the data in Phase 1.

\subsubsection{Phase 2}

- Two interviews each with employers, employees and those individual who sustained work-related SCIs were conducted during the pilot study. Interviews were conducted with persons not sampled for the main study. During these interviews the researcher 
determined the way the interviews would generate the data required to answer the relevant aims and objectives of the study; how questions would follow a logical sequence and could be pitched at the correct level for the respondents to understand easily; and approximately how long it would take to complete an interview. It took approximately one hour to conduct the interview. No changes were made to the questions.

\subsection{Data Collection Procedure}

\subsubsection{Phase 1}

- Permission to peruse the patient files from the two Life Health Care Units in Gauteng was obtained from Life Health Care National Standards Manager (See Appendix E).

- Codes from the International Coding of Diseases Version 10 coding system was used to identify all files of employees who sustained spinal cord injuries.

- All files in the Archives room were systematically perused to identify the relevant cases. Three hundred cases of SCI were identified. A further analysis was done to identify cases of SCI that occurred in the workplace during the study period.

- A case was identified through having Compensation of Occupational Injuries and Disease Act (COIDA) as the medical insurance on the sticker on the outside of the file and by perusing the medical record.

- Information from the identified folders was captured using the data coding form (Appendix A). This capturing was done twice by the researcher on separate forms and then compared by the statistician to ensure that the information had been captured correctly. Where discrepancies were found, the information was compared with that in the folder again and corrected by the researcher.

- The information from the data coding forms was captured onto SPSS Version 18.

It was anticipated that some of the required information would be missing from the files. Imputation was used to contain this problem if the missing data on a file was between 5-15\%. Imputation was used to allocate values for missing, incorrect or conflicting responses. The researcher used logical imputation, in which a consistent value is calculated from other information in the file; this is usually preferred over dynamic imputations (Community Survey SA, 2007). For instance where the neurological level and classification was absent logical imputation was done by deducing these from the findings of dermatome and myotome assessments.

- The data was coded and analysed using SPSS Version 18. 
- This process took five months to complete.

\subsubsection{Phase 2}

- Telephonic or personal contact was made with the prospective participants to obtain initial consent to conduct the interviews and arrange a date, time and venue for the interview.

- Before the researcher started the interview he explained the study to the participant, answered any questions the participant may have and asked the participant to sign the Informed Consent form. Consent was obtained at this stage to audio tape the interview.

- A voice recorder was used to capture all interviews. Interviews were performed in the participant's language of choice. The researcher is fluent in English, Afrikaans, IsiXhosa and Zulu and performed all interviews himself since no participant preferred to be interviewed in another language.

- The interviews were conducted by the researcher, in the language that the participant was comfortable with. A dictaphone (voice recorder) was used to capture the responses from the participants.

- On completion, the researcher asked the participant if s/he was agreeable to the researcher contacting them again should it become necessary to verify any data.

- However, this part of data collection proved to be difficult as the researcher experienced difficulties in receiving permission from the companies that were approached. The reluctance of the employers to participate in this study could be due to fear of being found to be non-complaint with health and safety regulations.

There are heavy punitive measures in place for employers who do not comply. It could be that the employers anticipated a breach of confidentiality when the findings of the results were reported, although this was clearly explained in the covering letter and that each participant would sign consent upon commencement of data collection.

\subsection{Data Analysis}

\subsubsection{Phase 1}

Two statistical tools were used: Excel (to create graphs) and SPSS Version 18 (to analyse data). These created tables and graphs that illustrate the demographics of this cohort, causes of work-related injuries at two rehabilitation centres in Gauteng, South Africa, the type of industry most affected, the occupation category most affected, and information about the injury. A calculation of the relationship between variables was determined using these tools. 
Graphs, frequency tables and histograms were used to present data such as age distribution, cause of injury, medical costs etc. Means, medians, standard deviations and quartiles were computed to describe the central tendency and variation of data. Various statistical tests e.g. ANOVA, KruskalWallis and the Chi square test (Domholdt, 2005) were used by the researcher under the guidance of the statistician to determine whether a statistically significant difference existed between variables. A p-value $<0.05$ was deemed statistically significant. Examples of data that were compared are type of industry versus number of injuries in that industry, type of industry versus cause of injury, type of industry versus level of injury, and day and time of injury versus number of injuries.

\subsubsection{Phase 2}

The recorded information was transcribed. To ensure accuracy, the researcher and research assistant worked separately to transcribe the information, then compared and discussed discrepancies to reach a consensus.

This was followed by content analysis during which the data from the interviews was analysed according to the following distinct, but interconnected, stages, as described by Rabie (2004):

- Familiarisation (through reading and re-reading transcripts, as well as through repeatedly listening to audio tapes)

- Identifying a thematic structure

- Indexing, charting and mapping and

- Analysis of data.

Attention was given to the non-verbal communication of interviewees. This was noted on the interview schedule during the interview and where non-verbal means of communication was evident on recordings (e.g. lengthy silences or laughter) it was added to transcripts. Data was presented according to identified themes and narrative examples were used to highlight these. Verification of data was done through member checking and doing interviews until data saturation occurred (Domholdt, 2005).

\subsection{Ethical Considerations}

- Ethical clearance was obtained from the University of Stellenbosch before the commencement of the study.

- Permission to conduct the study was obtained from the two Life Health Care Rehabilitation Units in Gauteng Province (Appendix E) and from the various companies identified. 
- Each participant was asked to sign an informed consent form in a language of their choice before the commencement of the interviews (Appendix D). The consent form was translated into other languages once the researcher knew which other languages study participants preferred.

- Participants had a right to withdraw from the study at any stage without fear of victimisation.

- The information gathered during the interview and from folders was kept in utmost confidentiality. Tapes and hard copies of data were stored in a locked safe for which only the researcher had the key. This data will be destroyed on completion of the thesis.

- Only the researcher and the statistician had access to the information for data analysis purposes.

- There were no names or other identifying particulars on the data collection tools. A participant number was allocated to each participant and each folder. Only these numbers were used on data collection tools. The identifying particulars of participants and folders were stored in a separate password-protected computer file.

- All information from the perused files was kept in strictest confidentiality. No names or any information that could divulge the identity of the participant were used during dissemination of the findings.

\subsection{Study Limitations}

- The study sample was small as only two rehabilitation facilities in Gauteng allowed the researcher access to peruse the files; thus results cannot be generalised to the general population of Gauteng.

- The Department of Labour (Compensation Fund) refused the researcher permission to peruse their files.

- This was a retrospective and institution-based study by comparison with population-based, prospective studies that have more weight in their ability to generalise the findings to the whole population.

- In some instances clinical data was missing or lacking in completeness as indicated under the presentation of ICF scores.

- The findings of the study can only be used in a similar setting to the research sites. Different provinces in South Africa have different types of industries.

- Secondary complications could have been one of the major factors in prolonged stay during in-patient rehabilitation and thus impacting on cost; however, secondary complications following SCIs were not explored in this study. 
- The researcher was refused permission from the companies approached to interview managers and employees in relation to issues of health and safety in the workplace. This perspective would have given some insight into current practices to form an opinion regarding future practices to prevent SCIs in the workplace.

- Interviewing only five participants for the qualitative phase proved to be too small a number. In addition the depth of data obtained was not sufficient.

- Educational levels of participants were not determined in both phases of the study.

\subsection{Chapter Summary}

The study adopted a mixed method approach in collecting the required data to answer the objectives. The study was therefore divided into two phases. Due to refusal of access to files by the Department of Labour, patient folders from two private rehabilitation facilities in Gauteng were used to collect data.

The population of the first phase of the study included all files of individuals who sustained SCI at work and presented with some neurological damage. For the second phase all individuals whose folders were perused formed the study population. A total of 140 files were identified of which five had to be excluded due to missing data, resulting in the perusal of 135 folders during data collection. Five individuals with work-related SCI were interviewed. No employers or employees could be interviewed since the researcher was refused access to the companies selected for the study.

A pilot study was done to assess the applicability of the data collection tools to collect all the necessary information.

Quantitative data from Phase 1was captured on Excel and SPSS Version 18 for statistical analysis. Qualitative data from Phase 2 was analysed using content analysis.

Ethical considerations were applied throughout the study. The next chapter focuses on the results of the study. 


\section{CHAPTER 4: QUANTITATIVE RESULTS}

\subsection{Introduction}

This chapter presents the quantitative results of the study. First the demographic profile of participants is described, followed by their occupational characteristics. Next the types, causes and severity of the spinal cord injuries (SCIs) sustained by participants are described. This is followed by an analysis of the costs of rehabilitation, which were estimated using the costs per day as stipulated by the Department of Labour. The nature of rehabilitation received is explored with regard to teamwork, areas addressed to improve function and assistive devices issued. Finally rehabilitation outcomes are presented through ICF and FIM+FAM scores.

All information presented in this chapter was gathered from the patients' hospital folders. One hundred and forty files were perused from both research sites. Of these five $(3.6 \%)$ had to be excluded due to missing information which would have impacted negatively on the overall study results. Thus a total of 135 folders were perused, meaning the sample size was 135 participants. 


\subsection{Demographic Information Of Participants}

\subsubsection{Age and gender distribution of participants}

Figure 4.1 summarises the age and gender distribution of participants. The most affected age range was $26-35$ with 52 participants (39\%) followed by the 36-45 age range with 33 (24\%) participants. These age groups were also the most affected within the genders. The least affected age group overall in both sexes was the range 56-65 years, with $10(7 \%)$ participants.

With regard to gender Figure 4.1 shows that males were in the majority with $87 \%$ of participants being males. Males were also in the majority in every age group. The male-to-female injury ratio was 6.69:1.

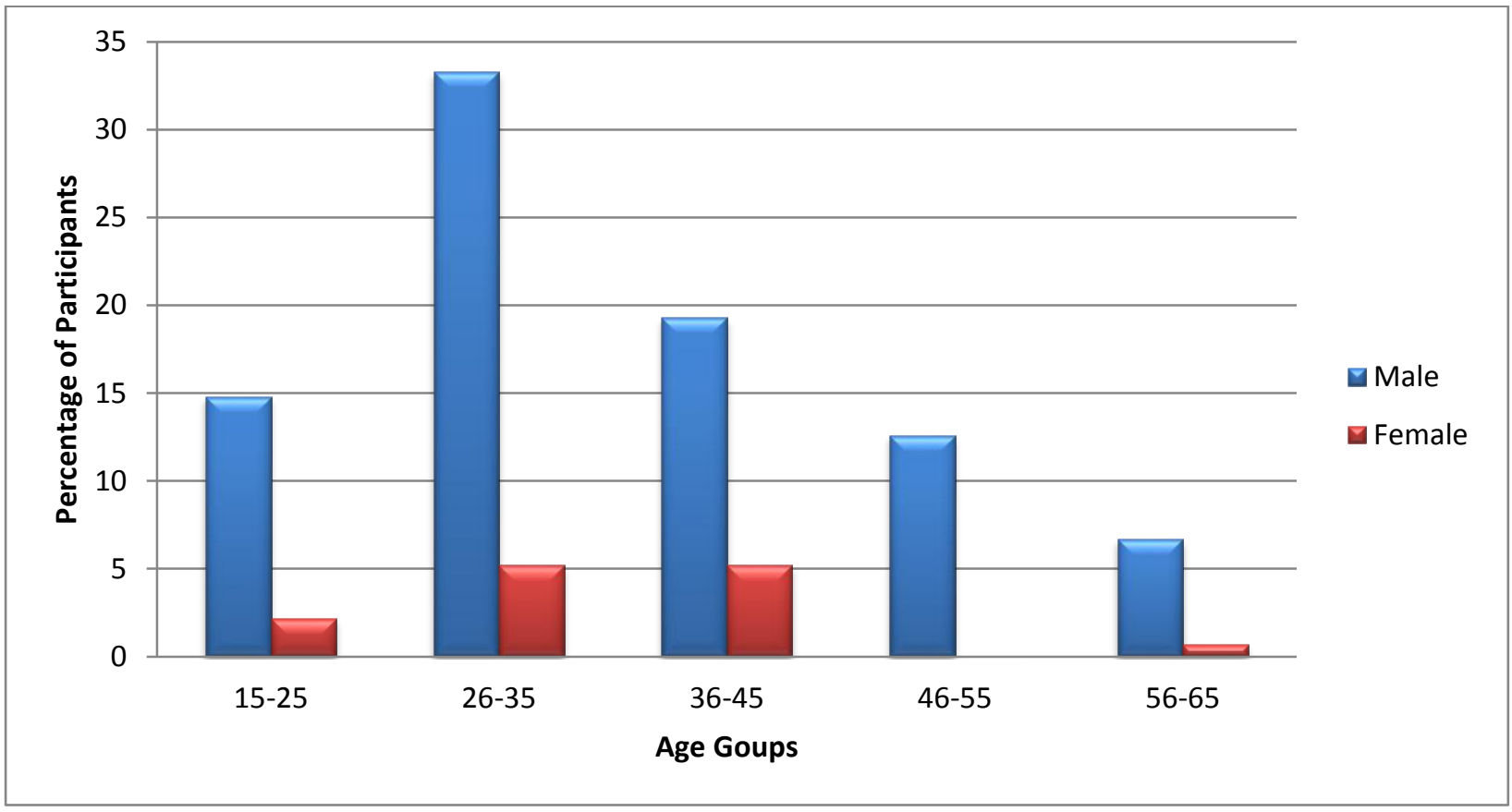

Figure 4.1 Gender and age distribution of participants 


\subsubsection{Marital status}

Figure 4.2 summarises the marital status of participants at the time of injury. The majority of participants were either married (52\%) or never married (44\%).

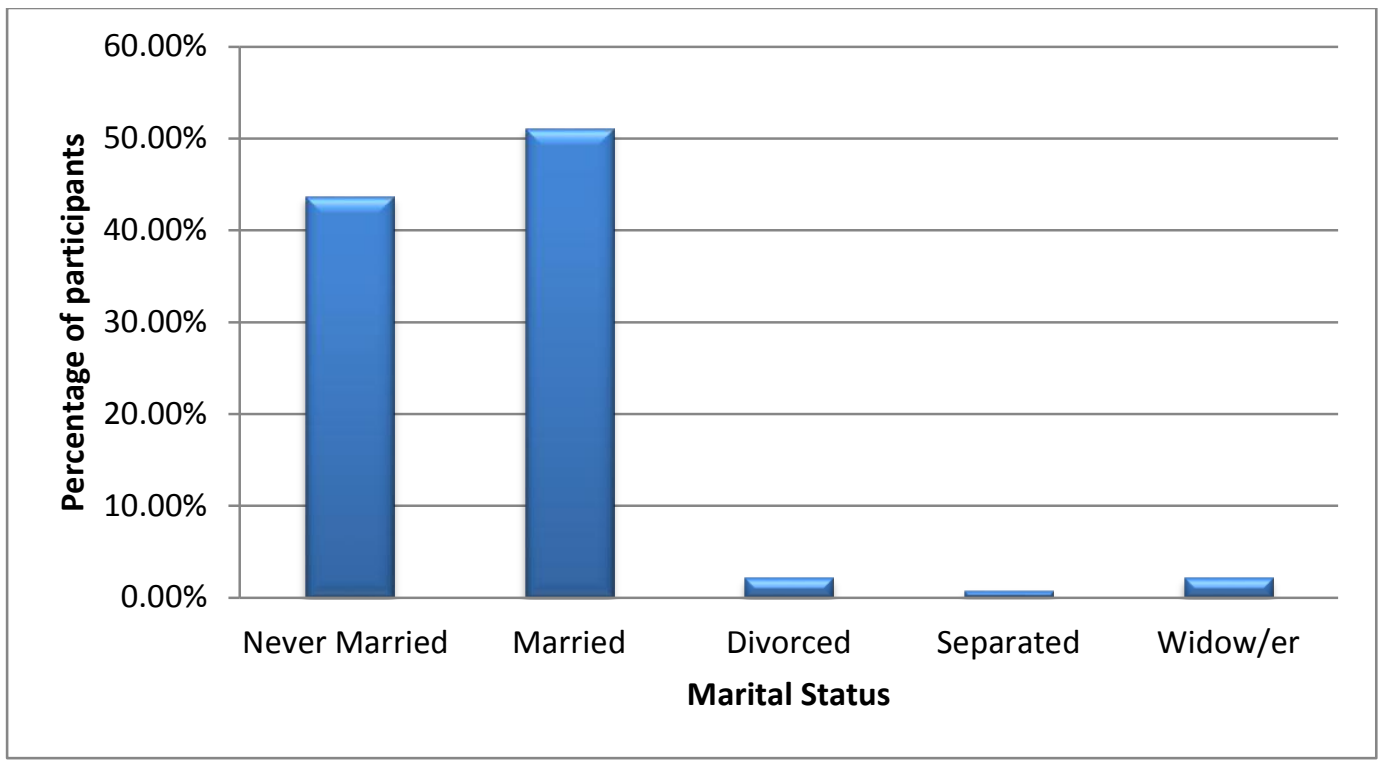

Figure 4.2 Marital status of participants 


\subsubsection{Population group and geographical distribution of places of employment}

Table 4.1 summarises by population group the geographical distribution of the places of employment of participants. Most SCIs occurred in urban areas (50\%) while the least occurred in rural areas (11\%). The highest number of injuries occurred amongst Blacks (79\%) while the least injuries occurred in the Indian population group (2\%).

Table 4.1 Population group and geographical location

\begin{tabular}{|c|c|c|c|c|c|c|}
\hline & & & \multicolumn{3}{|c|}{$\begin{array}{l}\text { Geographical location } \\
\text { (Where injury occurred) }\end{array}$} & \multirow[b]{2}{*}{ Total } \\
\hline & & & Urban & $\begin{array}{l}\text { Peri- } \\
\text { Urban }\end{array}$ & Rural & \\
\hline \multirow{4}{*}{$\begin{array}{l}\text { Population } \\
\text { group }\end{array}$} & Black & $\begin{array}{l}\text { Count } \\
\% \text { within population group } \\
\% \text { within geographical location } \\
\% \text { of Total }\end{array}$ & $\begin{array}{l}50 \\
47 \\
75 \\
37\end{array}$ & $\begin{array}{l}46 \\
43 \\
87 \\
34\end{array}$ & $\begin{array}{r}11 \\
10 \\
73 \\
8\end{array}$ & $\begin{array}{r}107 \\
100 \\
79 \\
79\end{array}$ \\
\hline & White & $\begin{array}{l}\text { Count } \\
\% \text { within population group } \\
\% \text { within geographical location } \\
\% \text { of Total }\end{array}$ & $\begin{array}{r}12 \\
60 \\
18 \\
9 \\
\end{array}$ & $\begin{array}{r}5 \\
25 \\
9 \\
4 \\
\end{array}$ & $\begin{array}{r}3 \\
15 \\
20 \\
2 \\
\end{array}$ & $\begin{array}{r}20 \\
100 \\
15 \\
15\end{array}$ \\
\hline & Coloured & $\begin{array}{l}\text { Count } \\
\% \text { within population group } \\
\% \text { within geographical location } \\
\% \text { of Total }\end{array}$ & $\begin{array}{r}3 \\
50 \\
5 \\
2\end{array}$ & $\begin{array}{r}2 \\
33 \\
4 \\
2\end{array}$ & \begin{tabular}{r|}
1 \\
17 \\
7 \\
0
\end{tabular} & $\begin{array}{r}6 \\
100 \\
4 \\
4\end{array}$ \\
\hline & Indian & $\begin{array}{l}\text { Count } \\
\% \text { within population group } \\
\% \text { within geographical location } \\
\% \text { of Total }\end{array}$ & $\begin{array}{r}2 \\
100 \\
3 \\
2\end{array}$ & $\begin{array}{l}0 \\
0 \\
0 \\
0\end{array}$ & $\begin{array}{l}0 \\
0 \\
0 \\
0\end{array}$ & $\begin{array}{r}2 \\
100 \\
2 \\
2\end{array}$ \\
\hline \multicolumn{2}{|l|}{ Total } & $\begin{array}{l}\text { Count } \\
\% \text { of Total }\end{array}$ & $\begin{array}{l}67 \\
50\end{array}$ & $\begin{array}{l}53 \\
39\end{array}$ & $\begin{array}{l}15 \\
11\end{array}$ & $\begin{array}{l}135 \\
100\end{array}$ \\
\hline
\end{tabular}




\subsection{Employment-Related Information}

\subsubsection{Occupation at time of injury and type of industries affected}

Table 4.2 summarises the occupations by industry that participants were employed in at the time of injury. The service industry (35\%) was the most affected followed by engineering (15\%), mining $(14 \%)$ and construction (10\%). The least affected industry was the manufacturing industry (4\%).

The most commonly affected occupations within the industries were labourers (26\%) followed by professionals (24\%). The least affected occupation was managers (7\%). A high number of professionals were affected in the service industry (30\%). Labourers were the most affected occupation within the construction (69\%), agriculture (78\%) and engineering (60\%). In the manufacturing industry drivers were mostly affected (60\%) and in the mining sector, the miners were mostly affected (74\%). In the service industry the security cluster (police and security officers) was mostly affected (55\%). 


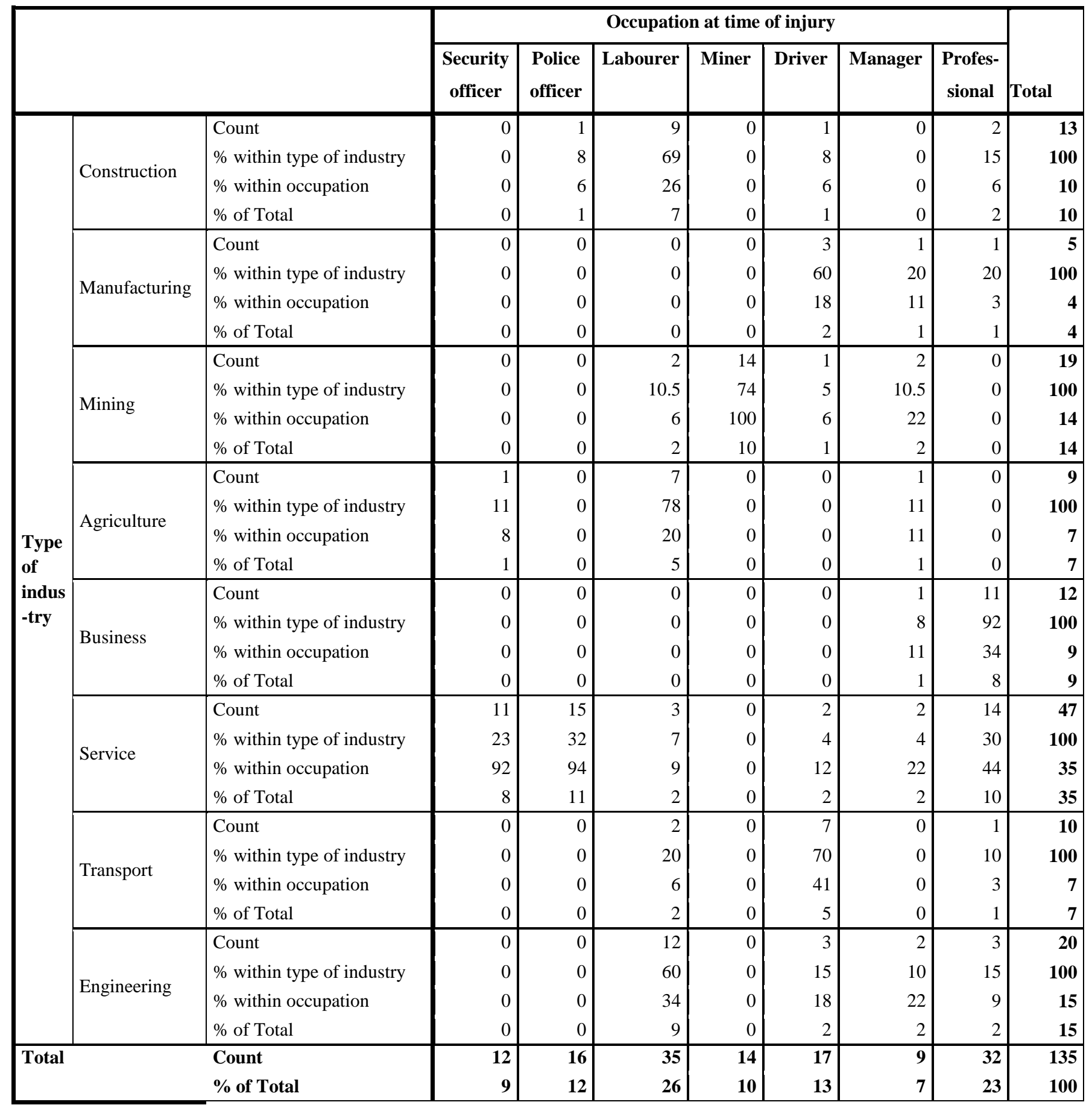




\subsubsection{Causes of work-related SCIs}

Figure 4.3 summarises the causes of the SCIs. The majority of injuries were caused by motor vehicle accidents (49\%) followed by violence (21\%) and falls (16\%).

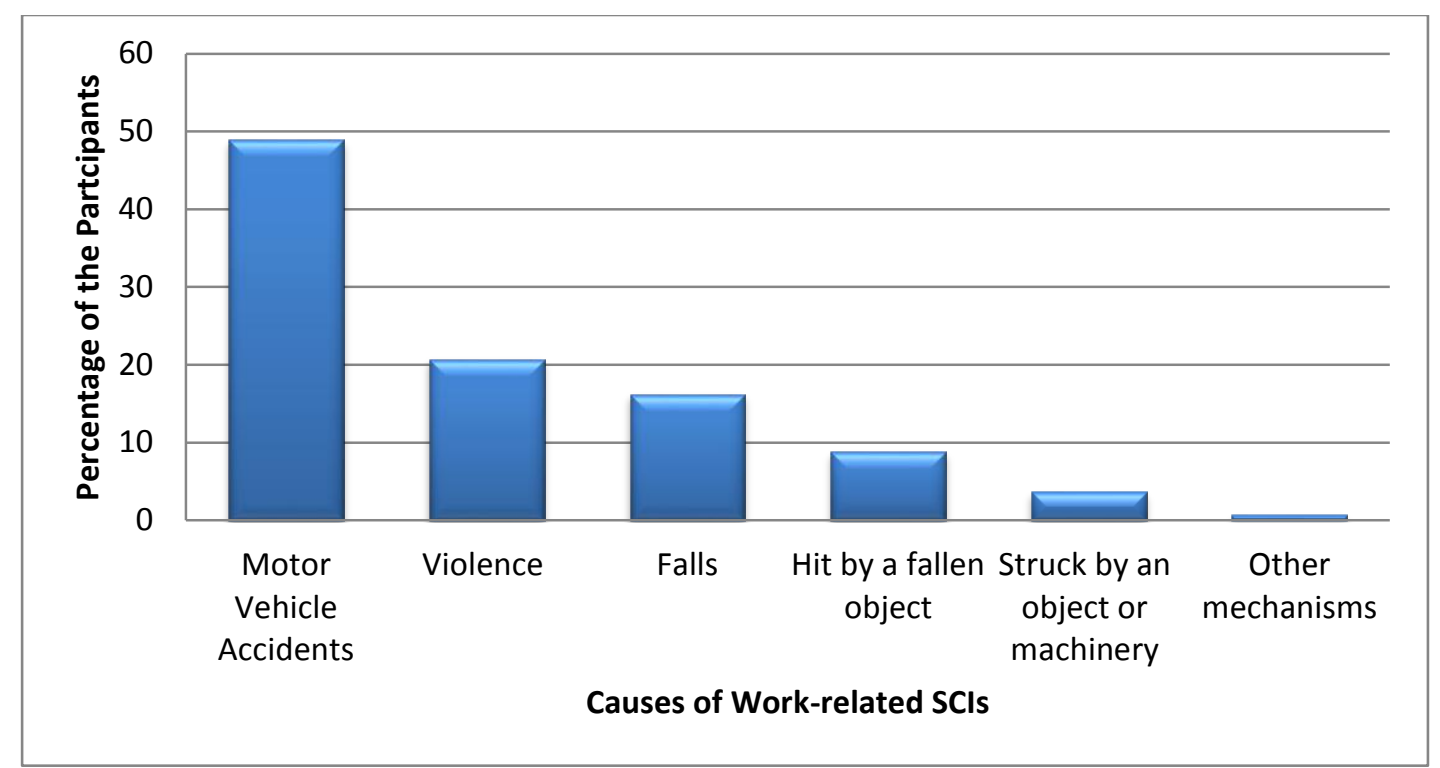

Figure 4.3 Causes of work-related SCIs 


\subsubsection{Motor vehicle accidents}

Table 4.3 summarises the types of motor vehicle accidents (MVAs) and crash types. Motor vehicle accidents where the injured individual was the driver counted for $60 \%$ of the injuries. Collision with another motor vehicle was the leading crash type (65\%) and collision with a roadside hazard (3\%) occurred least often.

Table 4.3 Type of vehicle, type of activity and type of accident

\begin{tabular}{|c|c|c|c|c|c|c|c|}
\hline & & & \multicolumn{4}{|c|}{ Crash Type } & \multirow[b]{2}{*}{ Total } \\
\hline & & & $\begin{array}{l}\text { Lost } \\
\text { control } \\
\text { and } \\
\text { rollover }\end{array}$ & $\begin{array}{c}\text { Collision } \\
\text { with } \\
\text { another } \\
\text { vehicle }\end{array}$ & $\begin{array}{c}\text { Collision } \\
\text { with a } \\
\text { roadside } \\
\text { hazard }\end{array}$ & $\begin{array}{l}\text { Collide } \\
\text { and } \\
\text { rollover }\end{array}$ & \\
\hline \multirow{12}{*}{$\begin{array}{l}\text { Types of } \\
\text { MVA and } \\
\text { relation of } \\
\text { injured } \\
\text { person to } \\
\text { motor } \\
\text { vehicle }\end{array}$} & \multirow{4}{*}{$\begin{array}{l}\text { Motor } \\
\text { vehicle } \\
\text { driver }\end{array}$} & Count & 6 & 23 & 2 & 8 & 39 \\
\hline & & $\%$ within type of MVA & 15 & 59 & 5 & 21 & 100 \\
\hline & & $\%$ within crash type & 60 & 55 & 100 & 73 & 60 \\
\hline & & $\%$ of Total & 9 & 35 & 3 & 12 & 60 \\
\hline & \multirow{4}{*}{$\begin{array}{l}\text { Motor } \\
\text { vehicle } \\
\text { passenger }\end{array}$} & Count & 4 & 14 & 0 & 1 & 19 \\
\hline & & $\%$ within type of MVA & 21 & 74 & 0 & 5 & 100 \\
\hline & & $\%$ within crash type & 40 & 33 & 0 & 9 & 29 \\
\hline & & $\%$ of Total & 6 & 22 & 0 & 2 & 29 \\
\hline & \multirow{4}{*}{$\begin{array}{l}\text { Motor } \\
\text { bike } \\
\text { driver }\end{array}$} & Count & 0 & 5 & 0 & 2 & 7 \\
\hline & & $\%$ within type of MVA & 0 & 71 & 0 & 29 & 100 \\
\hline & & $\%$ within crash type & 0 & 12 & 0 & 18 & 11 \\
\hline & & $\%$ of Total & 0 & 8 & 0 & 3 & 11 \\
\hline \multirow{2}{*}{\multicolumn{2}{|c|}{ Total }} & Count & 10 & 42 & 2 & 11 & 65 \\
\hline & & $\%$ of Total & 15 & 65 & 3 & 17 & 100 \\
\hline
\end{tabular}




\subsubsection{Types of violent activities}

Figure 4.4 shows that gunshot wounds (93\%) were the leading cause of violent SCIs in the workplace in the study population.

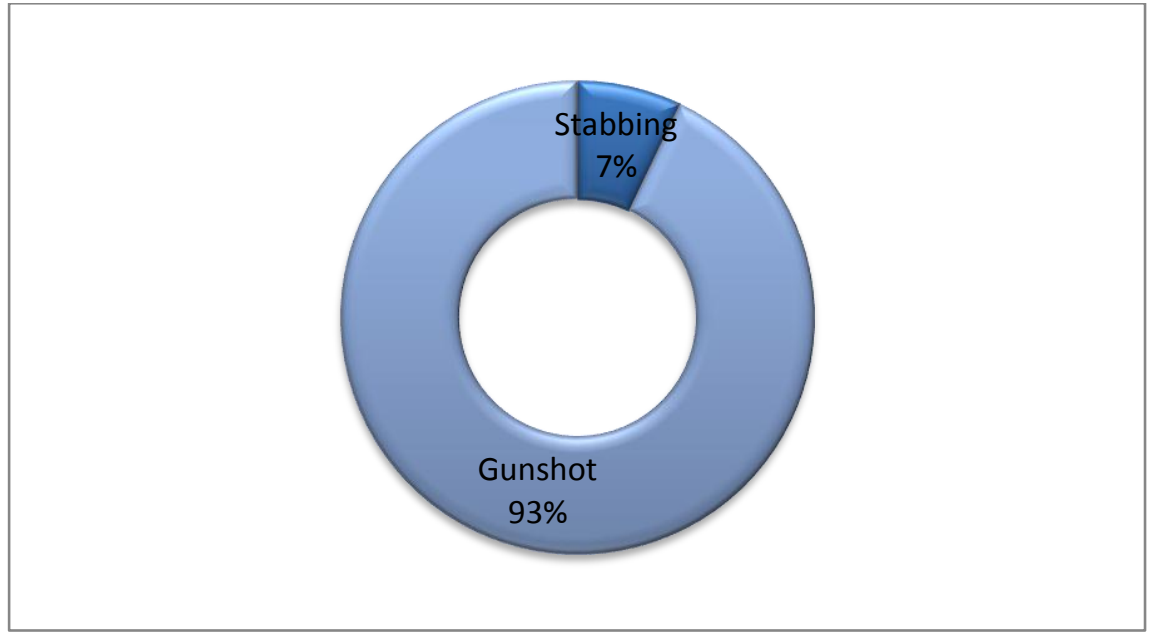

Figure 4.4 Types of violent activities that caused the SCI 


\subsubsection{Number of patients admitted per year}

Figure 4.5 presents the percentage of participants admitted to the study settings per year. There was a low admission rate between 2000 and 2001 (1\%). From 2001 to 2003 there was a smooth increase in the number of admissions at an average rate of 6\% per annum. Between 2005 and 2007 there was a decline at an average rate of $7 \%$ per annum. From 2007 to 2009 there was a sharp increase in the admission rate at an average rate of 18\% with a peak in 2009 (29\%). The average annual admission rate for the study period is $10 \%$ (13.5 individuals).

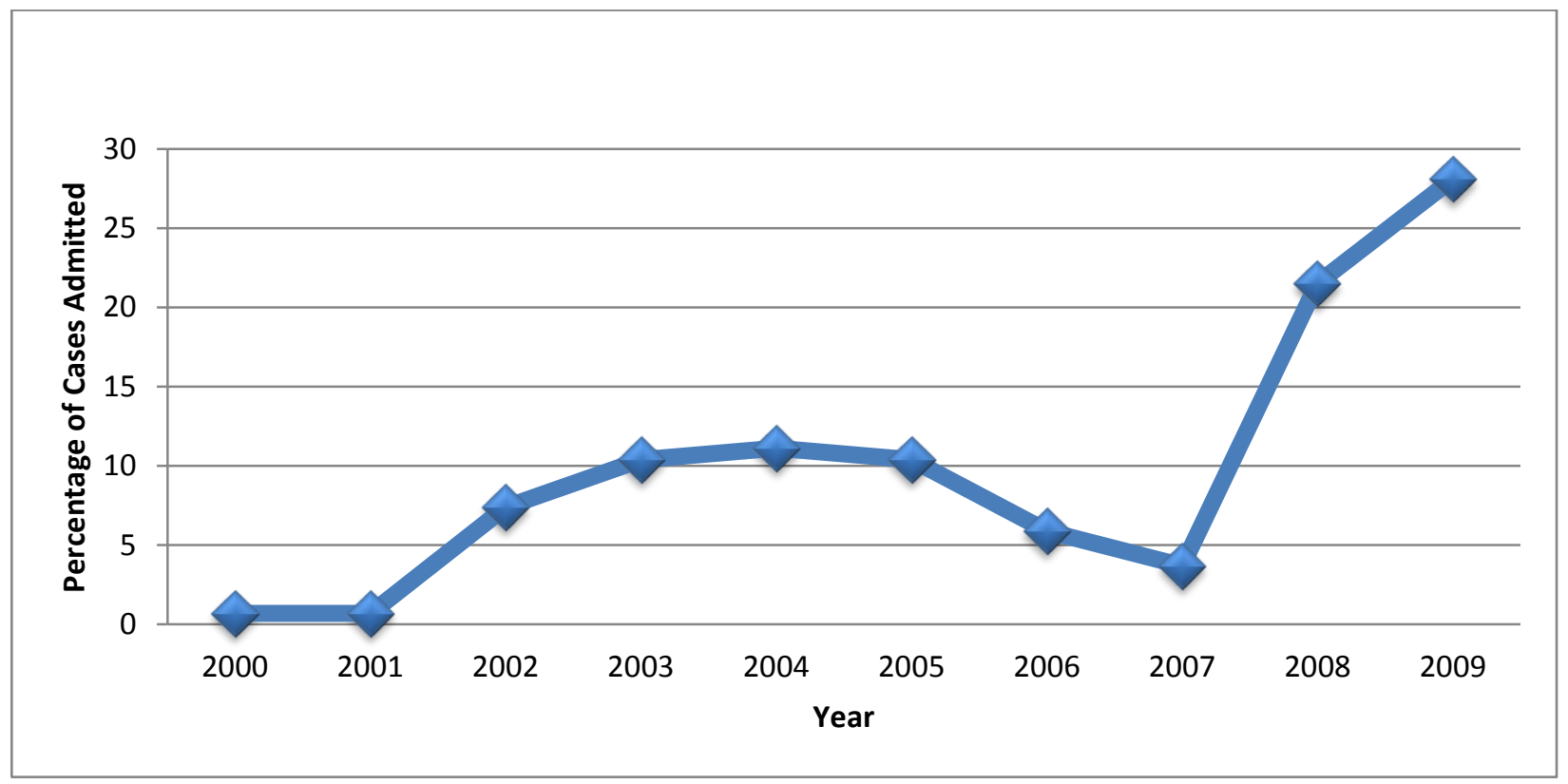

Figure 4.5 Number of patients admitted per year during study period 


\subsubsection{Time, day and month of injury}

Figure 4.6 shows the times when injuries occurred. The majority of injuries occurred during the day with $46 \%$ of the injuries occurring in the afternoon (12:01 to 18:00) and 33\% of the injuries occurring in the morning (06:01 to 12:00).

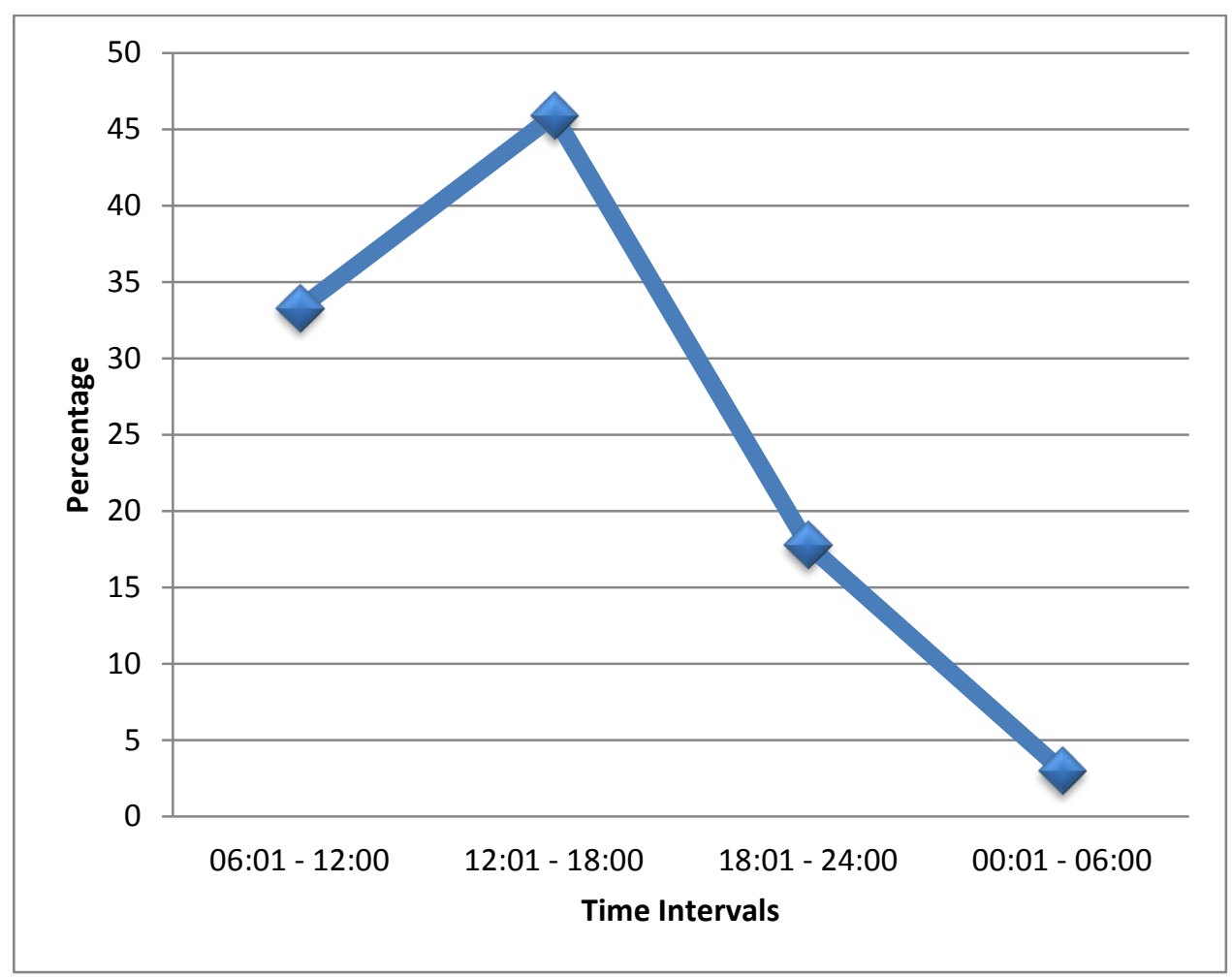

Figure 4.6 Time of injury 


\subsubsection{Injury according to time}

Table 4.4 summarises the most frequent causes of SCI according to the times they occurred. Most accidents (89\%) happened between 06:00 and 18:00 with the majority (46\%) of accidents occurring during the afternoons from 12:01 to 18:00.

Table 4.4 Causes of work-related SCI compared to time of injury

\begin{tabular}{|c|c|c|c|c|c|c|}
\hline & \multicolumn{4}{|c|}{ Time of Injury } & \multirow[b]{2}{*}{ Total } \\
\hline & & $\begin{array}{c}06: 01 \text { to } \\
12: 00\end{array}$ & $\begin{array}{c}12: 01 \text { to } \\
18: 00\end{array}$ & $\begin{array}{c}18: 01 \text { to } \\
24: 00\end{array}$ & $\begin{array}{c}\text { 00:01 to } \\
\text { 06:00 }\end{array}$ & \\
\hline $\begin{array}{l}\text { Motor Vehicle } \\
\text { Accident }\end{array}$ & $\begin{array}{l}\text { Count } \\
\% \text { within cause of injury } \\
\% \text { within time of injury } \\
\% \text { of Total }\end{array}$ & $\begin{array}{l}24 \\
36 \\
53 \\
18\end{array}$ & $\begin{array}{l}34 \\
52 \\
55 \\
25\end{array}$ & $\begin{array}{r}6 \\
9 \\
25 \\
4\end{array}$ & $\begin{array}{r}2 \\
3 \\
50 \\
2\end{array}$ & $\begin{array}{r}66 \\
100 \\
49 \\
49\end{array}$ \\
\hline Violence & $\begin{array}{l}\text { Count } \\
\% \text { within cause of injury } \\
\% \text { within time of injury } \\
\% \text { of Total }\end{array}$ & $\begin{array}{r}5 \\
18 \\
11 \\
4\end{array}$ & $\begin{array}{r}7 \\
25 \\
11 \\
5\end{array}$ & $\begin{array}{l}14 \\
50 \\
58 \\
10\end{array}$ & $\begin{array}{r}2 \\
7 \\
50 \\
2\end{array}$ & $\begin{array}{r}28 \\
100 \\
21 \\
21\end{array}$ \\
\hline Miscellaneous & $\begin{array}{l}\text { Count } \\
\% \text { within cause of injury } \\
\% \text { within time of injury } \\
\% \text { of Total }\end{array}$ & $\begin{array}{l}16 \\
39 \\
36 \\
12\end{array}$ & $\begin{array}{l}21 \\
51 \\
34 \\
16\end{array}$ & $\begin{array}{r}4 \\
10 \\
17 \\
3\end{array}$ & $\begin{array}{l}0 \\
0 \\
0 \\
0\end{array}$ & $\begin{array}{r}41 \\
100 \\
30 \\
30\end{array}$ \\
\hline Total & $\begin{array}{l}\text { Count } \\
\% \text { within cause of injury } \\
\% \text { within time of injury } \\
\% \text { of Total }\end{array}$ & \begin{tabular}{r|}
45 \\
33 \\
100 \\
33
\end{tabular} & \begin{tabular}{r|}
62 \\
46 \\
100 \\
46
\end{tabular} & $\begin{array}{r}24 \\
18 \\
100 \\
18\end{array}$ & \begin{tabular}{r|}
4 \\
3 \\
100 \\
3
\end{tabular} & $\begin{array}{l}135 \\
100 \\
100 \\
100\end{array}$ \\
\hline
\end{tabular}



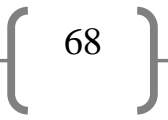

\subsubsection{Injuries per day of week}

Figure 4.7 presents the percentage of injuries per day of the week. The majority of the injuries occurred on Mondays and Thursdays (16\%), Fridays (17\%) and Sundays (16\%).

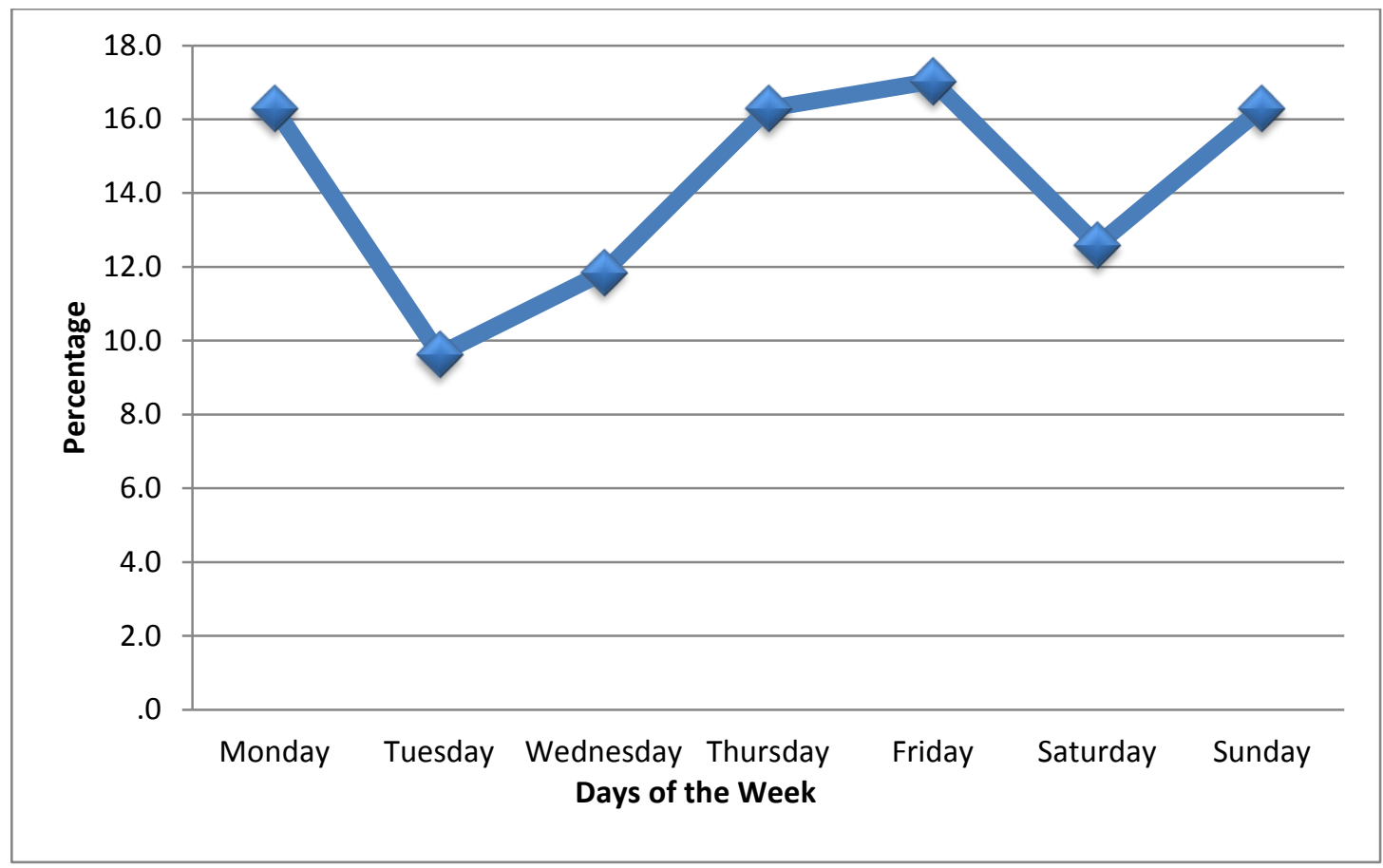

Figure 4.7 Day of injury 


\subsubsection{Injuries per month of occurrence}

Figure 4.8 depicts the months when work-related SCIs occurred during the study period. Most injuries occurred in the months of February and April (12\% per month) and least occurred during December $(3 \%)$.

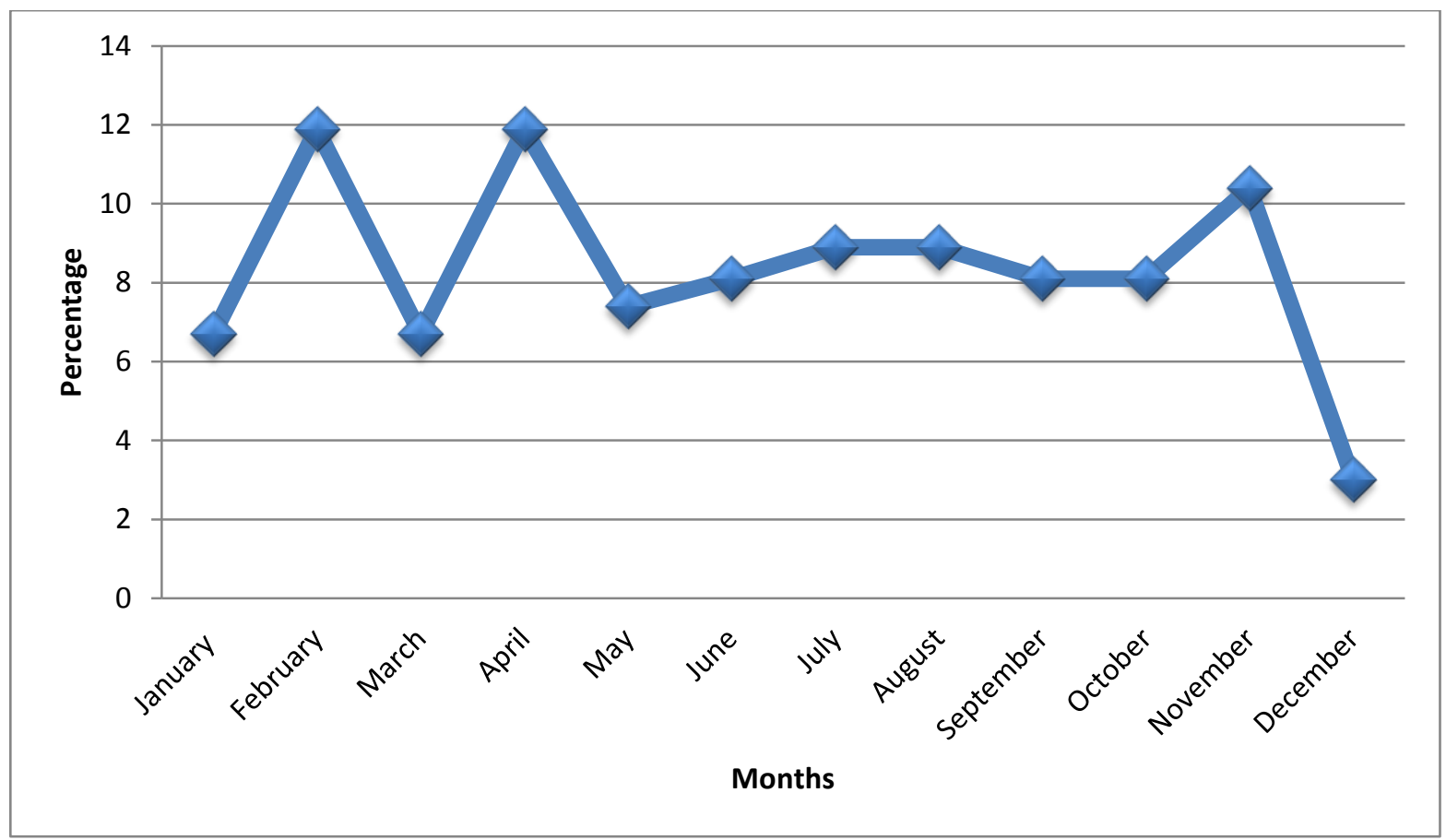

Figure 4.8 Month of injury 


\subsection{Level And Classification Of SCI}

The classification of SCIs with regard to completeness of injury was done according to the American Spinal Injury Association (ASIA) scale (Maynard et al., 1997), as presented in Chapter 2. In addition lesions were divided into cervical, thoracic or lumbar depending on the neurological level of the injury.

The information on level and classification of injury is summarised in Table 4.5. The most affected region of the spine was cervical (41\%), with the least affected region being the lumbar region (24\%). The majority (51\%) of participants had complete lesions (ASIA A). The majority of participants with incomplete lesions were classified as ASIA D (26\%).

Table 4.5 Neurological level and ASIA classification

\begin{tabular}{|r|r|r|r|r|r|}
\hline & ASIA-A & ASIA-B & ASIA-C & ASIA-D & \multicolumn{1}{|c|}{ Total } \\
\hline Cervical & 24 & 9 & 6 & 17 & $\mathbf{5 6}(\mathbf{4 1 \%})$ \\
\hline Thoracic & 32 & 2 & 5 & 8 & $\mathbf{4 7}(\mathbf{3 5 \%})$ \\
\hline Lumbar & 13 & 6 & 3 & 10 & $\mathbf{3 2}(\mathbf{2 4 \%})$ \\
\hline Total & & & & & \\
\hline
\end{tabular}


Table 4.6 compares the neurological levels to the causes of work-related SCIs. The majority of the injuries involving all the regions of the spine occurred in motor vehicle accidents. Although the biggest percentage of thoracic injuries was caused by motor vehicle accidents (16\%), violence (12\%) also played a big role as cause of thoracic injuries.

Table 4.6 Neurological level and cause of injury

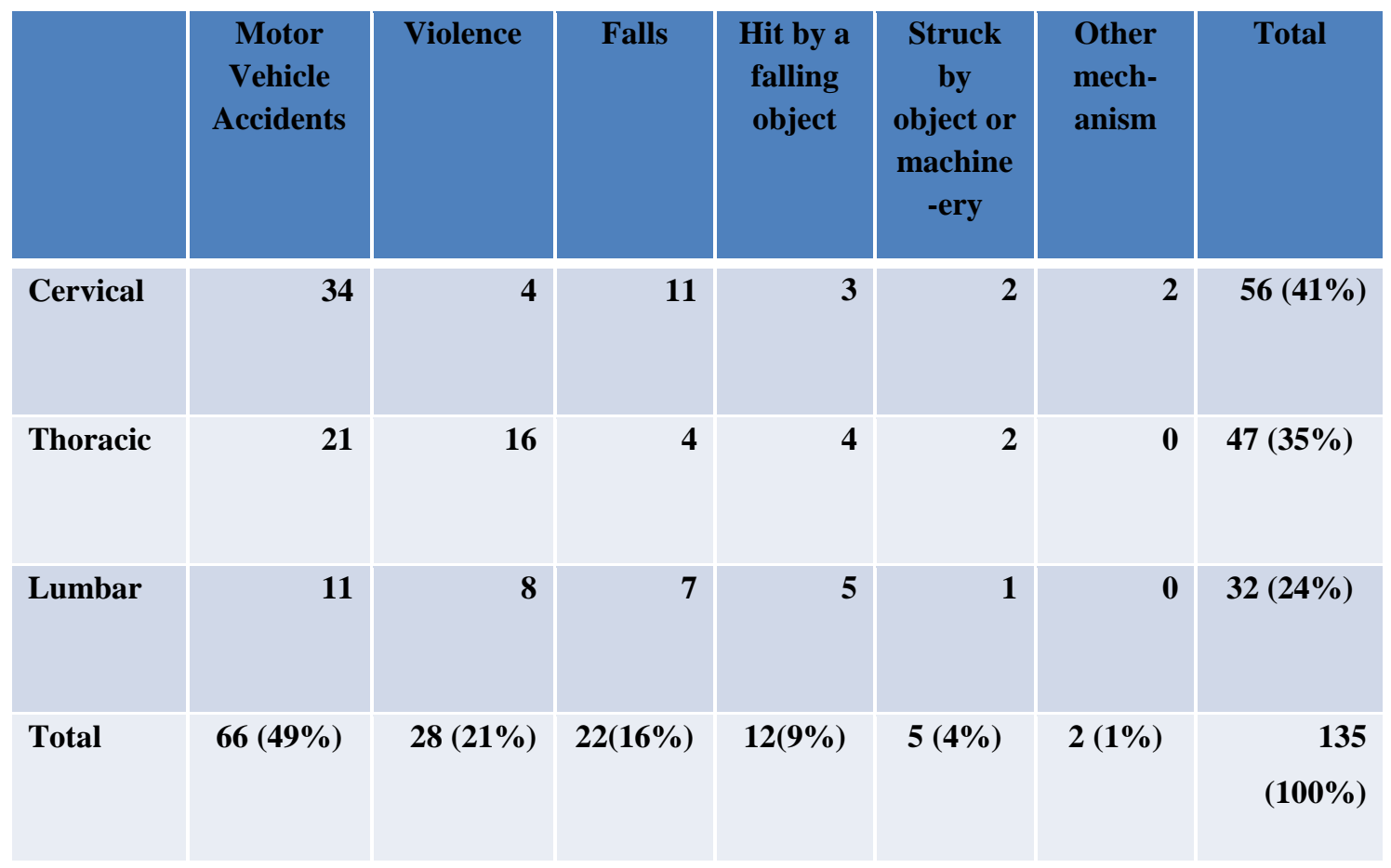


Table 4.7 presents the findings with regard to neurological levels compared to the different types of occupations of participants. The majority of security officers (50\%), labourers (43\%) and professionals (47\%) sustained injuries in the thoracic region of the spine. The majority of police officers (44\%), miners (57\%) and drivers (53\%) sustained injuries in the cervical region of the spine while the majority of managers (44\%) sustained injuries to the lumbar spine.

There was no statistically significant (Fishers Exact Test) relationship between neurological level and type of occupation $(\mathrm{p}=0.303)$.

Table 4.7 Occupation and neurological level

\begin{tabular}{|c|c|c|c|c|c|}
\hline & \multicolumn{3}{|c|}{$\begin{array}{c}\text { Neurological level (NL) } \\
\text { of the injury }\end{array}$} & \multirow[t]{2}{*}{ Total } \\
\hline & & Cervical & Thoracic & Lumbar & \\
\hline \multirow{4}{*}{ Security Officer } & Count & 4 & 6 & 2 & 12 \\
\hline & $\%$ within occupation & 33 & 50 & 17 & 100 \\
\hline & $\%$ within NL & 7 & 12 & 6 & 9 \\
\hline & $\%$ of Total & 3 & 4 & 2 & 9 \\
\hline \multirow{4}{*}{ Police Officer } & Count & 7 & 4 & 5 & 16 \\
\hline & $\%$ within occupation & 44 & 25 & 31 & 100 \\
\hline & $\%$ within NL & 13 & 9 & 16 & 12 \\
\hline & $\%$ of Total & 5 & 3 & 4 & 12 \\
\hline \multirow{4}{*}{ Labourer } & Count & 14 & 15 & 6 & 35 \\
\hline & $\%$ within occupation & 40 & 43 & 17 & 100 \\
\hline & $\%$ within NL & 25 & 32 & 19 & 26 \\
\hline & $\%$ of Total & 10 & 11 & 4 & 26 \\
\hline \multirow{4}{*}{ Miner } & Count & 8 & 1 & 5 & 14 \\
\hline & $\%$ within occupation & 57 & 7 & 36 & 100 \\
\hline & $\%$ within NL & 14 & 2 & 16 & 10 \\
\hline & $\%$ of Total & 6 & 1 & 4 & 10 \\
\hline \multirow{4}{*}{ Driver } & Count & 9 & 4 & 4 & 17 \\
\hline & $\%$ within occupation & 53 & 23.5 & 23.5 & 100 \\
\hline & $\%$ within NL & 16 & 9 & 13 & 13 \\
\hline & $\%$ of Total & 7 & 3 & 3 & 13 \\
\hline \multirow{4}{*}{ Manager } & Count & 3 & 2 & 4 & 9 \\
\hline & $\%$ within occupation & 33 & 22 & 45 & 100 \\
\hline & $\%$ within NL & 5 & 4 & 13 & 7 \\
\hline & $\%$ of Total & 2 & 1 & 3 & 7 \\
\hline \multirow{4}{*}{ Professional } & Count & 11 & 15 & 6 & 32 \\
\hline & $\%$ within occupation & 34 & 47 & 19 & 100 \\
\hline & $\%$ within NL & 20 & 32 & 19 & 24 \\
\hline & $\%$ of Total & 8 & 11 & 4 & 24 \\
\hline \multirow{5}{*}{ Total } & Count & 56 & 47 & 32 & 135 \\
\hline & $\%$ within & 41 & 35 & 24 & 100 \\
\hline & occupation & & & & \\
\hline & $\%$ within NL & 100 & 100 & 100 & 100 \\
\hline & $\%$ of Total & 41 & 35 & 24 & 100 \\
\hline
\end{tabular}


Table 4.8 (this page and next) compares the neurological level to the type of industry. Injuries to the cervical region of the spine were most common in the transport (70\%), mining (53\%) and construction (46\%) industries. In the engineering (45\%) and business (67\%) industries thoracic injuries were most common. The majority of the cervical as well as thoracic injuries occurred in the service industry, respectively accounting for $13 \%$ and $13 \%$ of the total number of participants. There was no statistically significant relationship (Fishers Exact Test) between the neurological level and the type of industry $(\mathrm{p}=0.745)$.

Table 4.8 Type of industry and neurological level

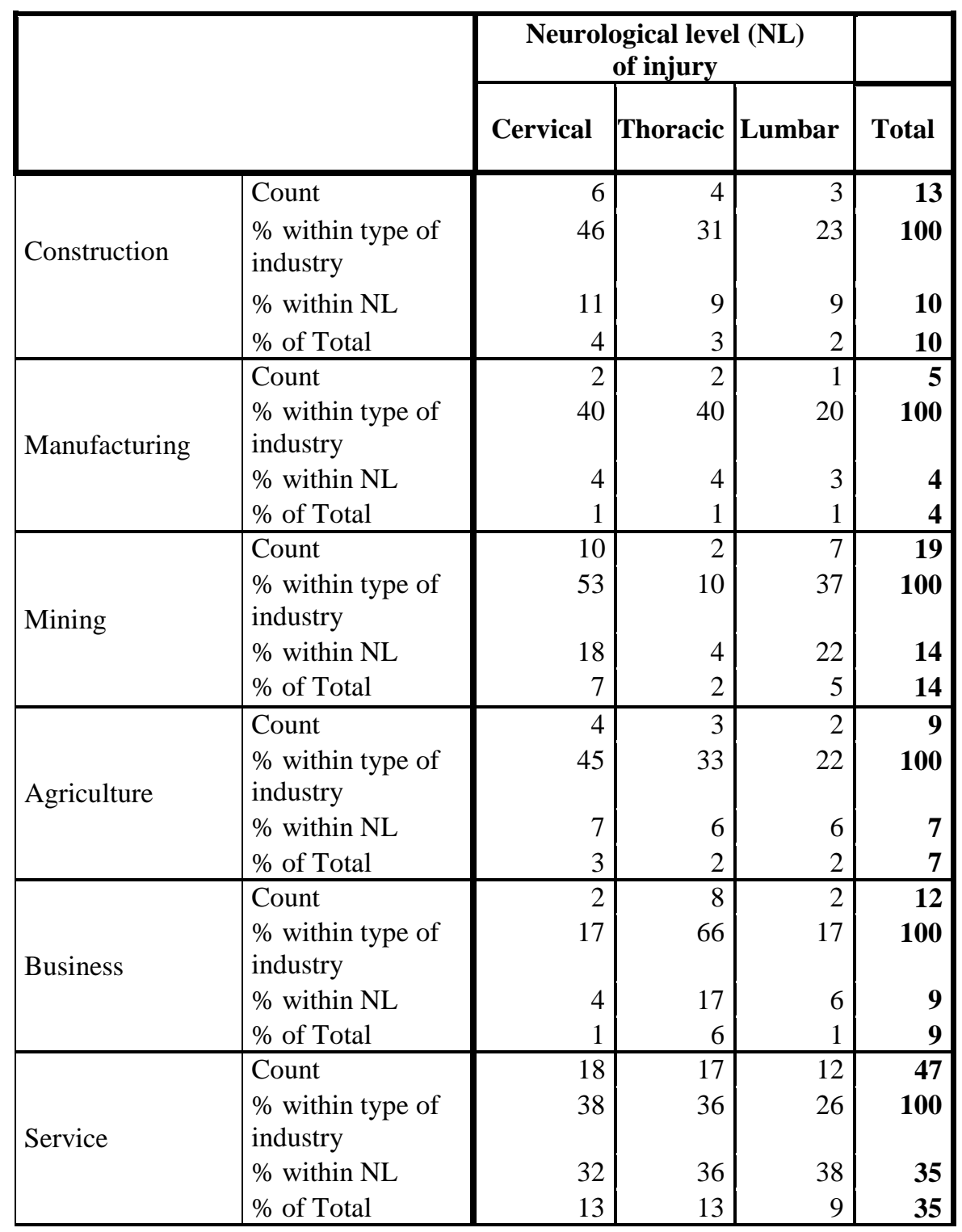


Table 4.8 Type of industry and neurological level (continued)

\begin{tabular}{|c|c|c|c|c|c|}
\hline & \multicolumn{3}{|c|}{$\begin{array}{c}\text { Neurological level (NL) } \\
\text { of injury }\end{array}$} & \multirow[b]{2}{*}{ Total } \\
\hline & & Cervical & Thoracic & Lumbar & \\
\hline \multirow{4}{*}{ Transport } & Count & 7 & 2 & 1 & 10 \\
\hline & $\begin{array}{l}\% \text { within type of } \\
\text { industry }\end{array}$ & 70 & 20 & 10 & 100 \\
\hline & $\%$ within NL & 13 & 4 & 3 & 7 \\
\hline & $\%$ of Total & 5 & 1 & 1 & 7 \\
\hline \multirow{4}{*}{ Engineering } & Count & 7 & 9 & 4 & 20 \\
\hline & $\begin{array}{l}\% \text { within type of } \\
\text { industry }\end{array}$ & 35 & 45 & 20 & 100 \\
\hline & $\%$ within NL & 13 & 19 & 12 & 15 \\
\hline & $\%$ of Total & 5 & 7 & 3 & 15 \\
\hline \multirow{5}{*}{ Total } & Count & 56 & 47 & 32 & 135 \\
\hline & $\%$ within type & 41 & 35 & 24 & 100 \\
\hline & of industry & 100 & 100 & 100 & \\
\hline & of WitnII NL & 100 & $\begin{array}{r}100 \\
35\end{array}$ & 100 & 100 \\
\hline & 8001 rotal & 41 & 55 & 24 & 100 \\
\hline
\end{tabular}




\subsection{Nature Of Rehabilitation Received}

The two study sites are inpatient rehabilitation units and as such all participants received in-patient rehabilitation. The nature of rehabilitation was explored in terms of which professions were represented in the teams, type of interventions provided and areas addressed as well as assistive devices provided.

\subsubsection{Team members}

At both units the rehabilitation team consisted of a medical practitioner with a special interest in rehabilitation, a physiotherapist, an occupational therapist, a social worker and a dietician. In $46.7 \%$ of cases participants were also referred to a neuropsychologist.

\subsubsection{Approaches to rehabilitation provided}

Figure 4.9 summarises the different types of rehabilitation approaches used by the rehabilitation teams in the study settings. As indicated (87\%) received family education and training, while all received individual therapy and $98 \%$ received group therapy as well.

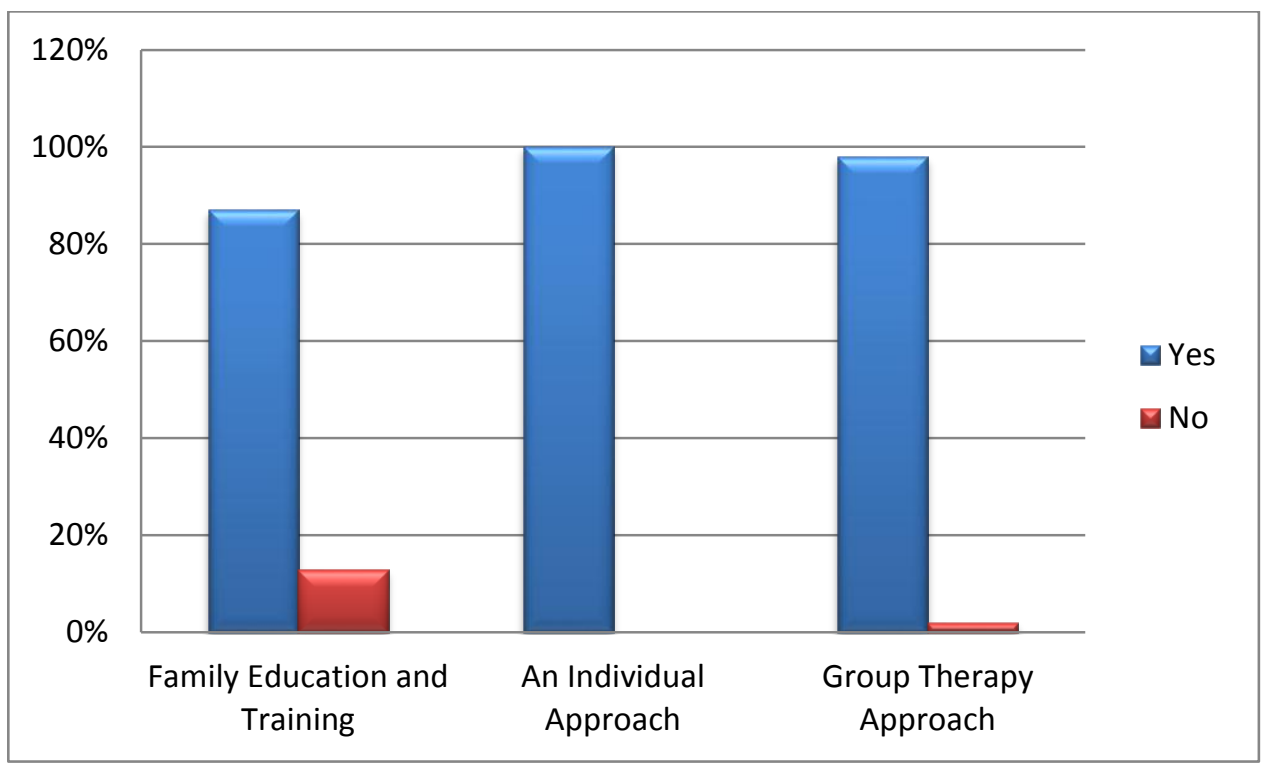

Figure 4.9 Approaches to rehabilitation 


\subsubsection{Types of rehabilitation services}

Figure 4.10 summarises the types of rehabilitation services provided to individuals with SCIs in the study settings. All participants received medical rehabilitation, diet therapy and counselling, while $0.7 \%$ of participants received vocational rehabilitation.

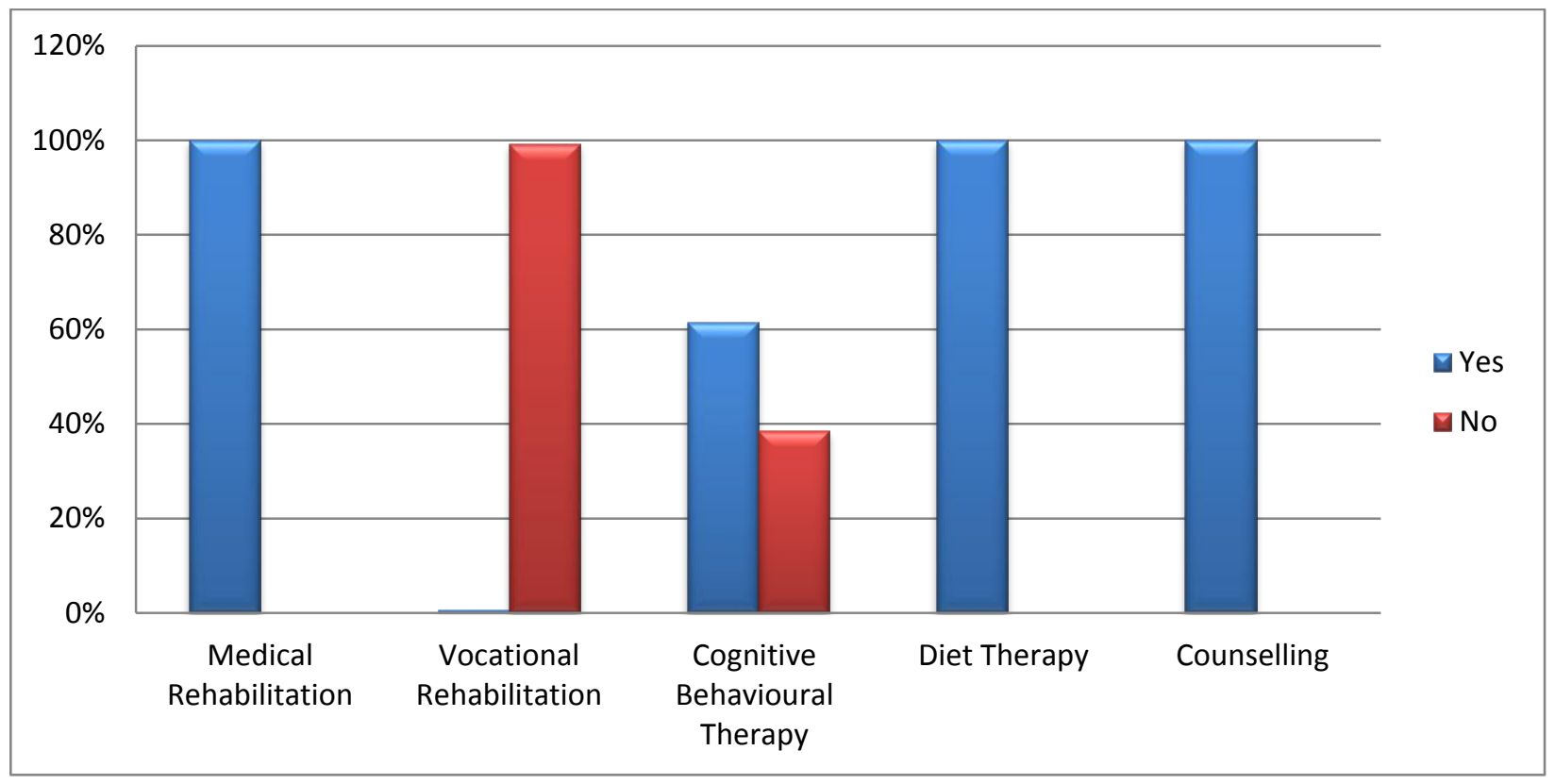

Figure 4.10 Types of rehabilitation services 


\subsubsection{Types of medical rehabilitation}

Figure 4.11 summarises the different types of medical rehabilitation provided to the participants. All participants were trained in wheelchair dexterity while $98 \%$ were taught transfers and therapeutic standing. Eighty four per cent of participants received gait re-education.

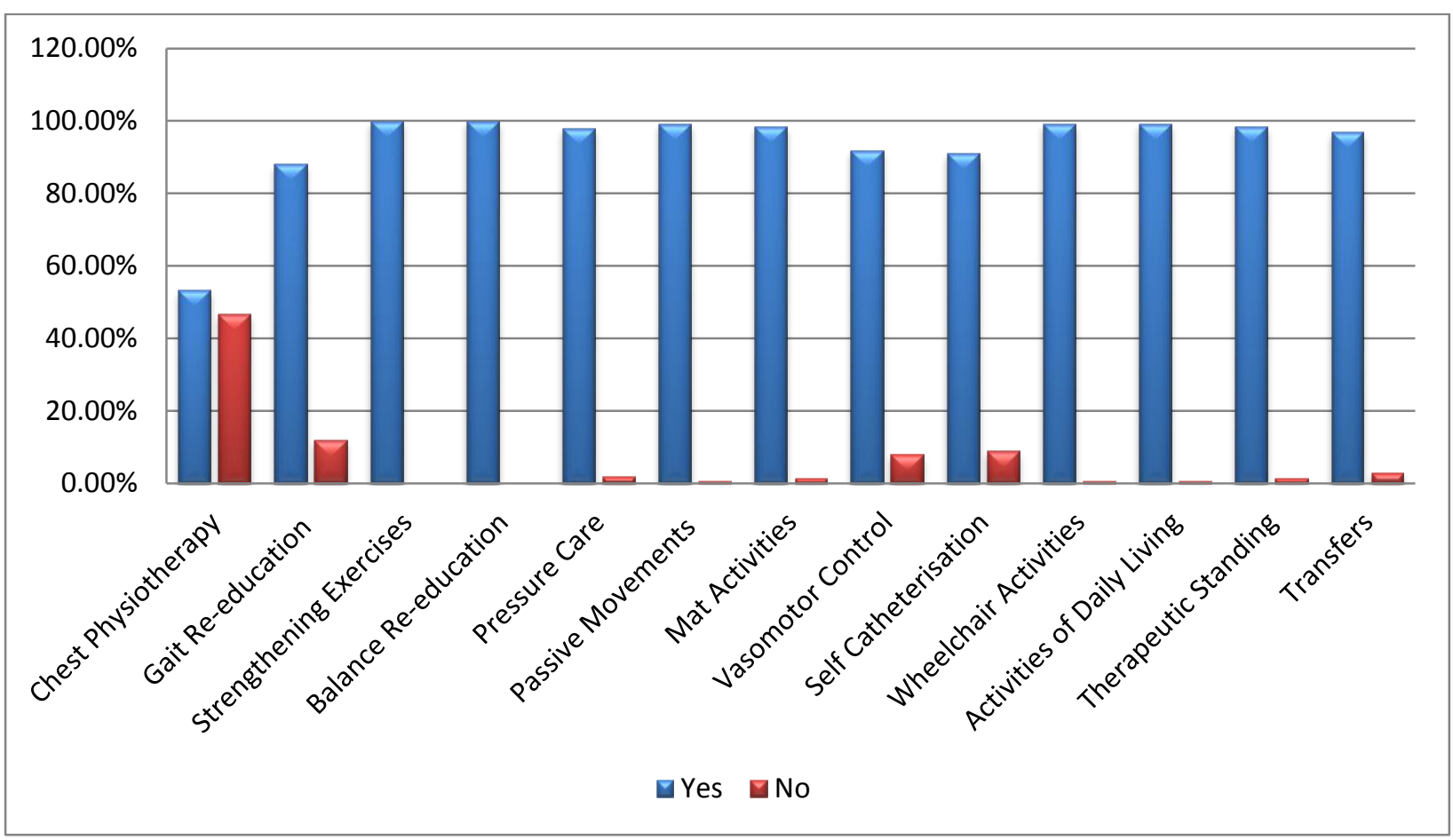

Figure 4.11 Types of medical rehabilitation 


\subsubsection{Assistive devices issued during rehabilitation}

\subsubsection{Assistive devices related to mobility}

Assistive devices issued during the period of rehabilitation were divided into two categories for further clarification i.e. mobility and those related to activities of daily living. Figure 4.12 summarises mobility-related assistive devices received during the rehabilitation period. The mobility devices most often issued were wheelchairs (69\%) and wheelchair cushions (67\%), while ramps were provided the least often (3\%).

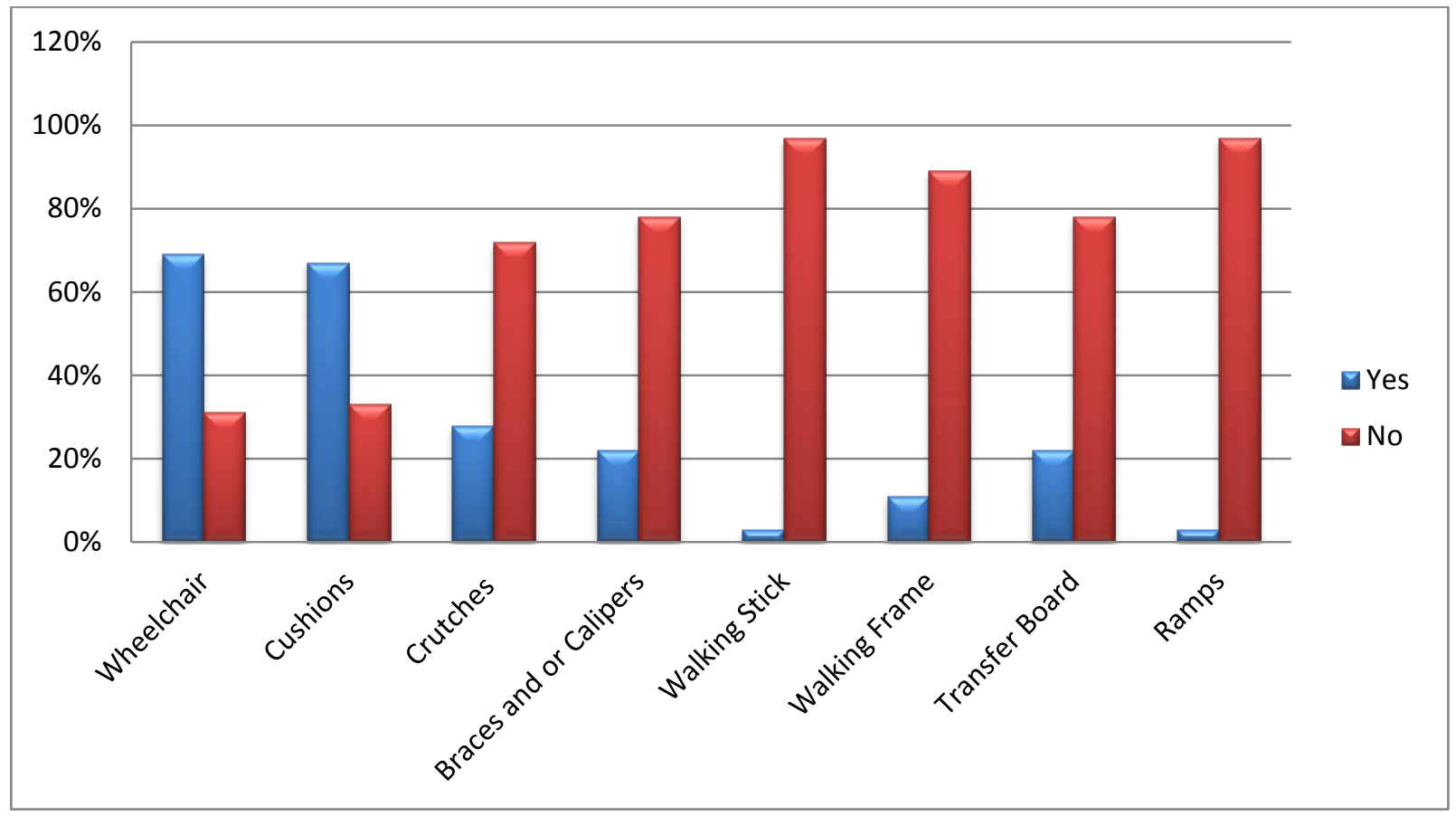

Figure 4.12 Assistive devices issued related to mobility 


\subsubsection{Assistive devices related to activities of daily living}

Figure 4.13 summarises the assistive devices related activities of daily living that were issued. The majority of the individuals did not receive any devices to assist with activities of daily living. Commodes (24\%) were the most often issued and splints (2\%) the least.

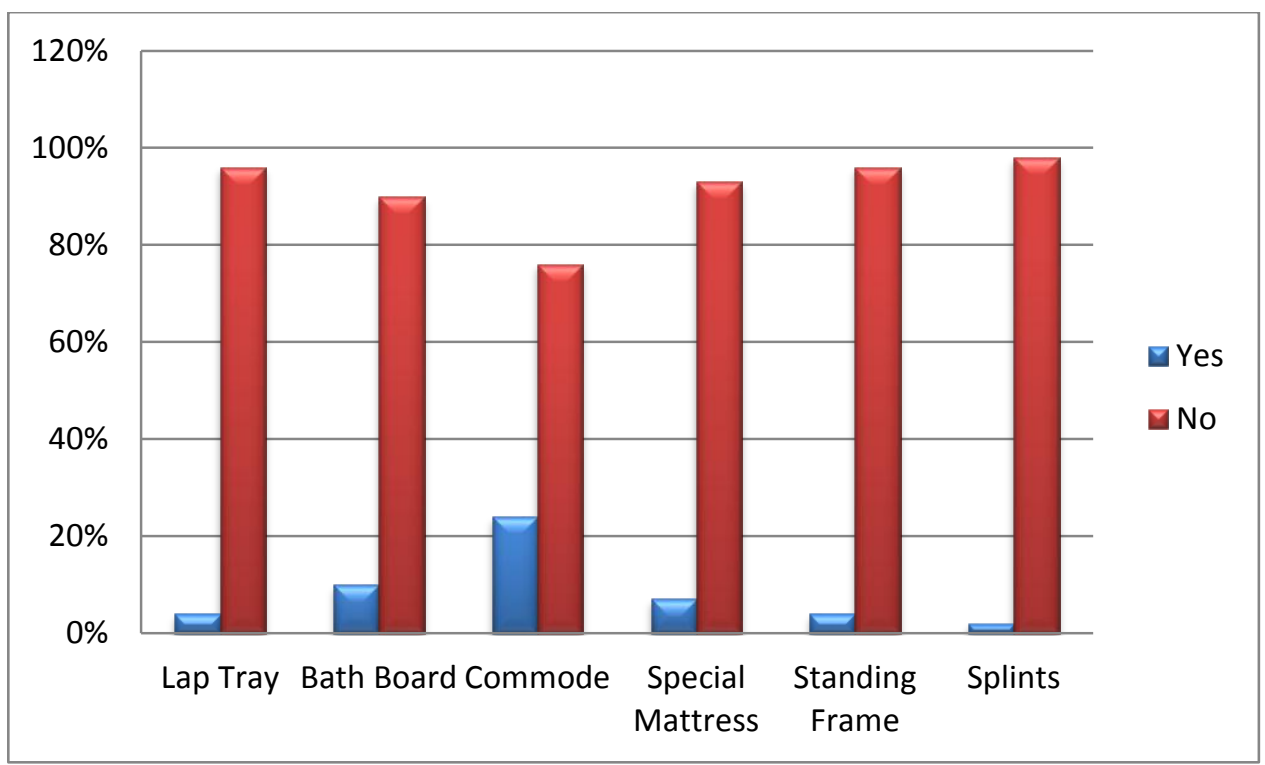

Figure 4.13 Assistive devices related to activities of daily living 


\subsubsection{Length of stay}

The length of stay (LOS) during rehabilitation was calculated for each participant by calculating the number of days between the admission and discharge date. The days where an individual was discharged for a home visit in preparation for discharge were included in the calculation as the patient was still under the authority of the rehabilitation facility.

Table 4.9 summarises the average length of stay per neurological level and shows a steady decline in length of stay from cervical injuries (72 days) through to lumbar injuries (52 days).

Table 4.9 Average LOS per neurological level

\begin{tabular}{|l|r|r|r|r|}
\hline $\begin{array}{l}\text { Neurological } \\
\text { Level }\end{array}$ & \multicolumn{1}{|c|}{ Mean } & \multicolumn{1}{|c|}{ Range } & \multicolumn{1}{|c|}{$\begin{array}{c}\text { Std. } \\
\text { Deviation }\end{array}$} \\
\hline Cervical & 56 & 71.68 & $10-204$ & 53.495 \\
\hline Thoracic & 47 & 67.98 & $15-350$ & 52.018 \\
\hline Lumbar & 32 & 52.13 & $9-156$ & 30.918 \\
\hline Total & $\mathbf{1 3 5}$ & $\mathbf{6 5 . 7 6}$ & $\mathbf{9 - 3 5 0}$ & $\mathbf{4 8 . 8 4 0}$ \\
\hline
\end{tabular}


Table 4.10 summarises the average LOS per ASIA classification. The average length of stay for the study population was 65.76 days with a standard deviation of 48.8. Patients who were classified as ASIA C stayed longer (85.64 days) in rehabilitation while participants with ASIA D stayed the shortest (38.69 days). The minimum days stayed in rehabilitation were 9 days (ASIA D) and the maximum 350 days (ASIA C).

Table 4.10 Length of stay and ASIA classification

\begin{tabular}{|c|c|c|c|c|c|}
\hline $\begin{array}{l}\text { Classification of } \\
\text { Injury } \\
\text { (According to the } \\
\text { ASIA scale) }\end{array}$ & $\mathbf{N}$ & Mean & $\begin{array}{c}\text { Std. } \\
\text { Deviation }\end{array}$ & Minimum & Maximum \\
\hline ASIA A & 69 & 72.42 & 41.030 & 12 & 204 \\
\hline ASIA B & 17 & 78.06 & 58.655 & 10 & 201 \\
\hline ASIA C & 14 & 85.64 & 84.568 & 19 & 350 \\
\hline ASIA D & 35 & 38.69 & 24.449 & 9 & 101 \\
\hline Total & 135 & 65.76 & 48.840 & 9 & 350 \\
\hline
\end{tabular}

Figure 4.14 summarises the length of stay per year. In 2001 the participants stayed on average the longest (173 days). There was a sharp decline, thereafter stabilizing at between 50 and 82 days.

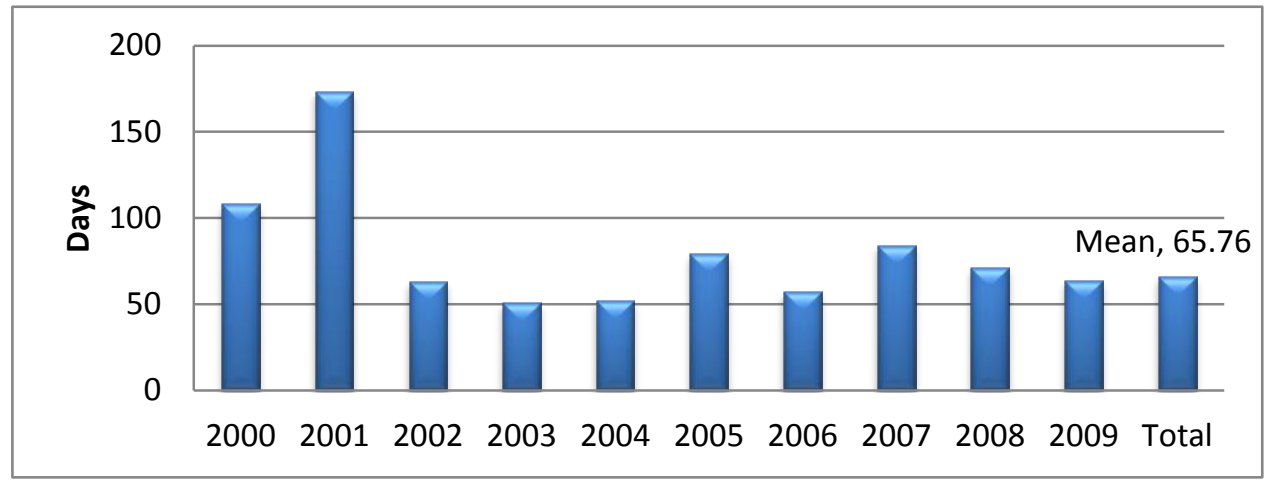

Figure 4.14 Average length of stay per year 
The Spearman correlation (r) was utilised to measure if a relationship between LOS and FIM+FAM scores (Functional and Cognitive) existed as the data were not normally distributed. According to Figure 4.15 there was a marginal correlation (linear relationship) between the length of stay and functional FIM+FAM scores on discharge ( $\mathrm{r}=-.0 .32)$, indicating that the longer the LOS the higher the FIM+FAM scores. Whereas Figure 4.16 showed no relationship between LOS and cognitive FIM+FAM scores $(r=0.06)$.

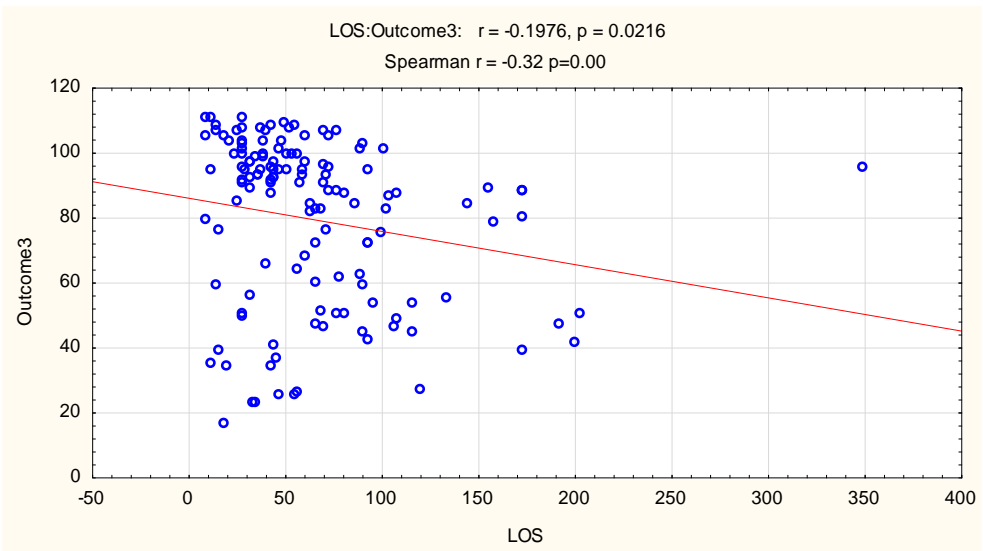

Figure 4.15 Length of stay compared to functional FIM+FAM score

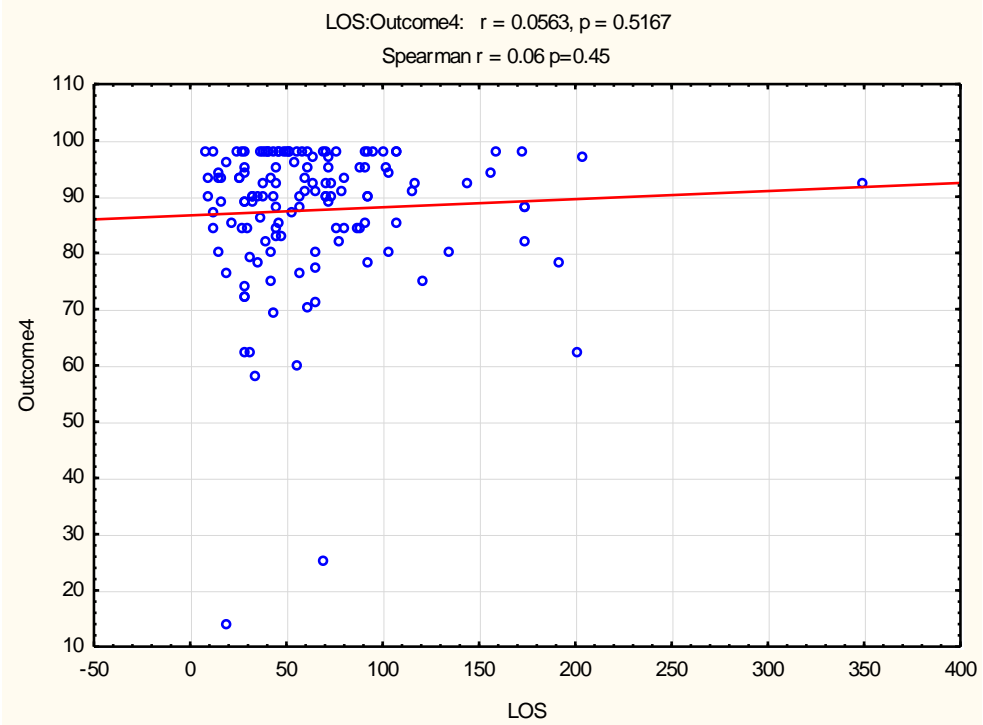

Figure 4.16 Length of stay compared to cognitive FIM+FAM score 
The Kruskal-Wallis test was used to statistically measure the correlation between LOS and the cause of injury, as more than two groups were observed. Figure 4.17 indicates no statistically significant correlation $(\mathrm{p}=0.12)$ between LOS and the different causes of SCIs amongst the participants. Participants who sustained SCIs through violence (2) showed the widest range of LOS and those who suffered from miscellaneous injuries (falls, hit or struck by an object) showed an overall shorter LOS with a mean of 57 days compared to the 70 days of the other two groups.

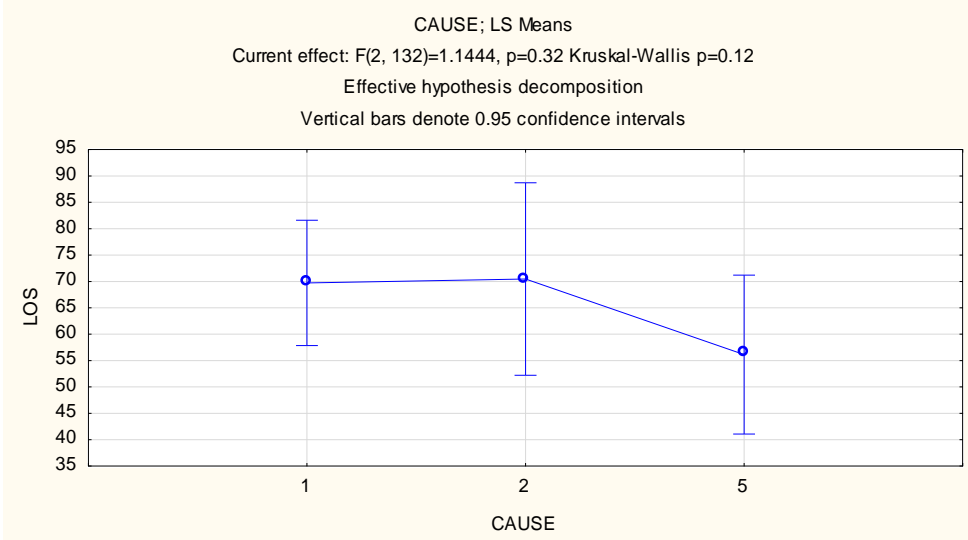

Figure 4.17 Impact of cause of injury on length of stay

The Mann-Whitney U test was used to measure the relationship between LOS and gender. There were no statistically significant differences between LOS for the two groups $(\mathrm{p}=0.41)$. The mean LOS for both groups was similar, but females showed a much wider distribution of LOS scores in Figure 4.16.

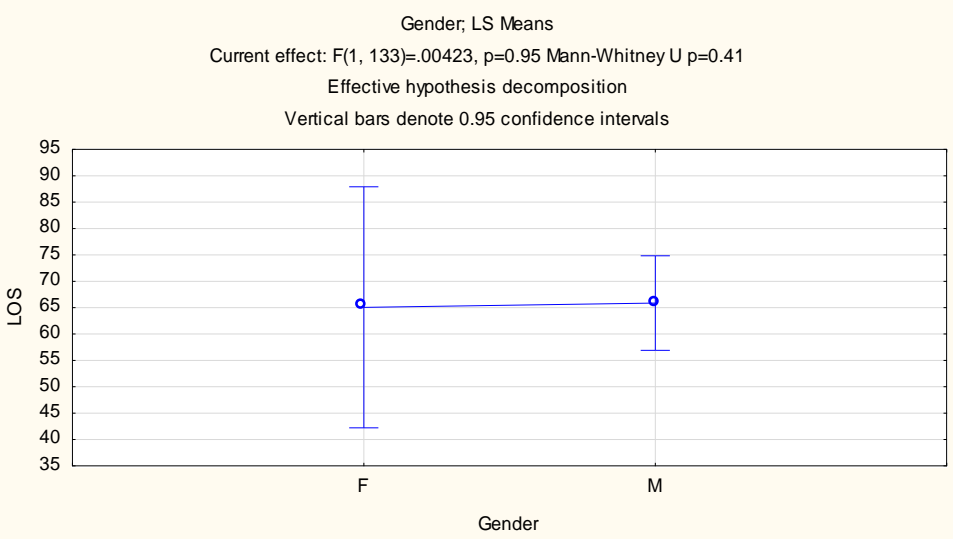

Figure 4.18 Impact of gender on length of stay 


\subsection{Costs Of Rehabilitation}

Costs of rehabilitation were estimated using costs per day as stipulated by the Department of Labour. This figure changes each year and is gazetted. Due to unavailability of the specific tariff for rehabilitation, the private ward fee as stipulated in the Scale of Fees for Private Hospital Gazette was used.

Estimated costs of rehabilitation were determined by multiplying the LOS and the private ward fee. It therefore excludes costs of assistive devices and medication. Table 4.11 summarises the average (mean) costs of rehabilitation per neurological level. The first two rows represent number of participants and the mean cost in Rands, followed by the standard deviation per neurological level. The most expensive neurological level to rehabilitate was the cervical region with an average cost of R116 956,00 and a standard deviation of 94 270,94. The least expensive level was the lumbar region at R78 075,40 with a standard deviation of 47583,43 .

Table 4.11 Estimated costs per neurological level (ZAR)

\begin{tabular}{|l|r|c|c|}
\hline $\begin{array}{c}\text { Neurological level } \\
\text { of injury }\end{array}$ & N & Mean & $\begin{array}{c}\text { Std. } \\
\text { Deviation }\end{array}$ \\
\hline Cervical & 56 & 116956.0000 & 94270.94321 \\
\hline Thoracic & 47 & 98707.9787 & 72818.58819 \\
\hline Lumbar & 32 & 78075.4063 & 47583.43186 \\
\hline Total & $\mathbf{1 3 5}$ & $\mathbf{1 0 1 3 8 6 . 8 4 4 4}$ & $\mathbf{7 8 9 0 0 . 7 2 9 4 7}$ \\
\hline
\end{tabular}

Table 4.11 indicates that the ASIA classifications with the highest mean cost were ASIA B and C.

Table 4.12 Estimated costs of rehabilitation and ASIA classification

\begin{tabular}{|l|r|r|r|r|r|}
\hline $\begin{array}{c}\text { Classification of } \\
\text { injury (according to } \\
\text { ASIA scale) }\end{array}$ & \multicolumn{1}{|c|}{$\mathbf{N}$} & \multicolumn{1}{c|}{ Mean } & \multicolumn{1}{c|}{$\begin{array}{c}\text { Std. } \\
\text { Deviation }\end{array}$} & Minimum & Maximum \\
\hline ASIA A & 69 & 108987.5652 & 62932.37581 & 15348.00 & 319464.00 \\
ASIA B & 17 & 142017.9412 & 1.21262 & 13460.00 & 431433.00 \\
ASIA C & 14 & 120379.0000 & 1.20029 & 25574.00 & 495950.00 \\
ASIA D & 35 & 59070.6000 & 35374.33766 & 12790.00 & 145638.00 \\
Total & 135 & 101386.8444 & 78900.72947 & 12790.00 & 495950.00 \\
\hline
\end{tabular}


Figure 4.19 depicts the average amount spent per year per participant. The average costs per year between year 2000 and 2001 was stable at below R120 000 per participant. There was a smooth decline thereafter (2002 to 2004). There was an increase in 2005 (just below R140 000 per participant) and a decline in 2006. From 2007 there was a steady decline until 2009.

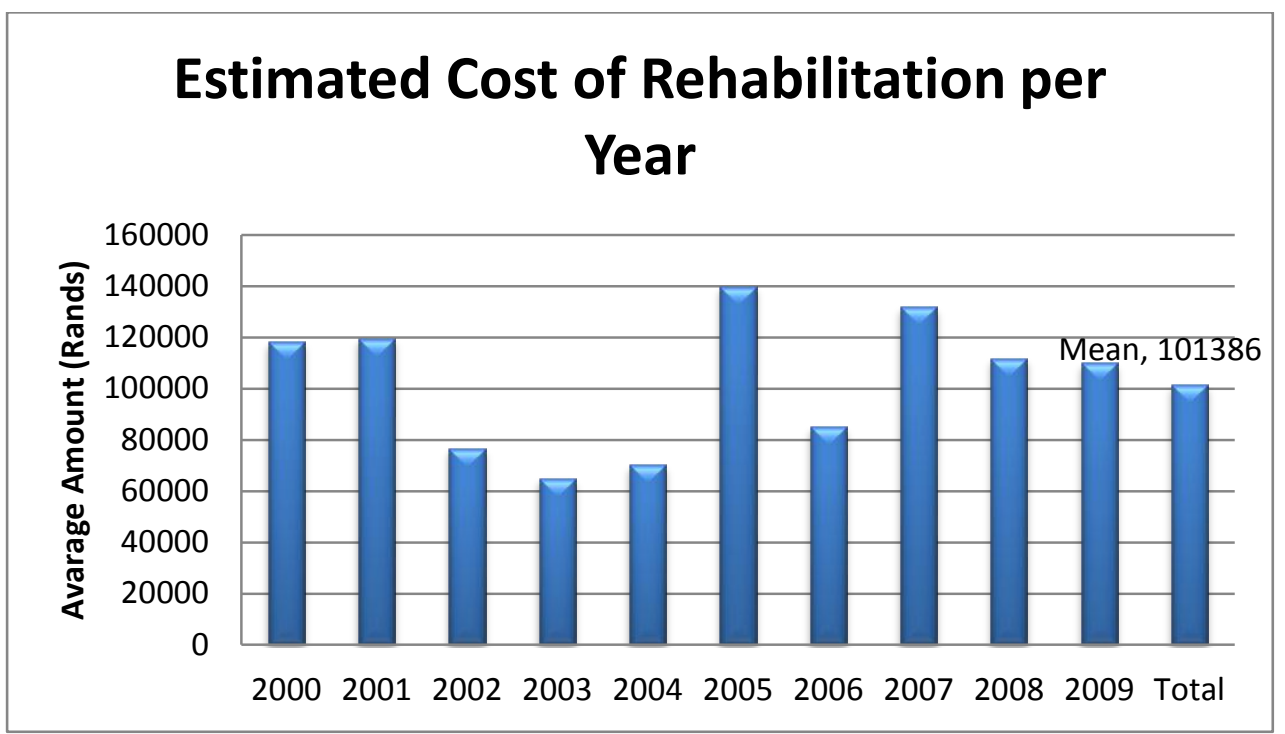

Figure 4.19 Average cost of rehabilitation per participant per year 


\subsection{Rehabilitation Outcomes}

Rehabilitation outcomes are presented through the patients' ICF activity and participation scores in the various ICF categories as well as a comparison of FIM+FAM admission and discharge scores. The ICF scores were determined by the researcher from the discharge reports compiled by rehabilitation professionals. The 1\% score of "not applicable" that is indicated in all graphs refers to one participant whose ICF scores could not be deduced because of insufficient information in the folder. The researcher realised that "not applicable" is not the optimal way to handle this issue, but still wanted to use the other data from this participant.

\subsubsection{Communication ICF scores}

The majority of participants had no difficulty in all areas of communication: $97 \%$ of participants had no difficulty with expressing themselves, $95 \%$ could start and maintain a conversation, and $93 \%$ could converse using devices and $90 \%$ could read, write and calculate. 


\subsubsection{Activities of daily living}

Figure 4.20 shows the ICF scores of participants related to activities of daily living. In this category participants experienced some challenges and while between 60 and $70 \%$ could perform most of the activities 20-30\% experienced various levels of difficulty with these activities. The most challenging area was staying alone at home for few days: $10 \%$ of participants could not do this at all.

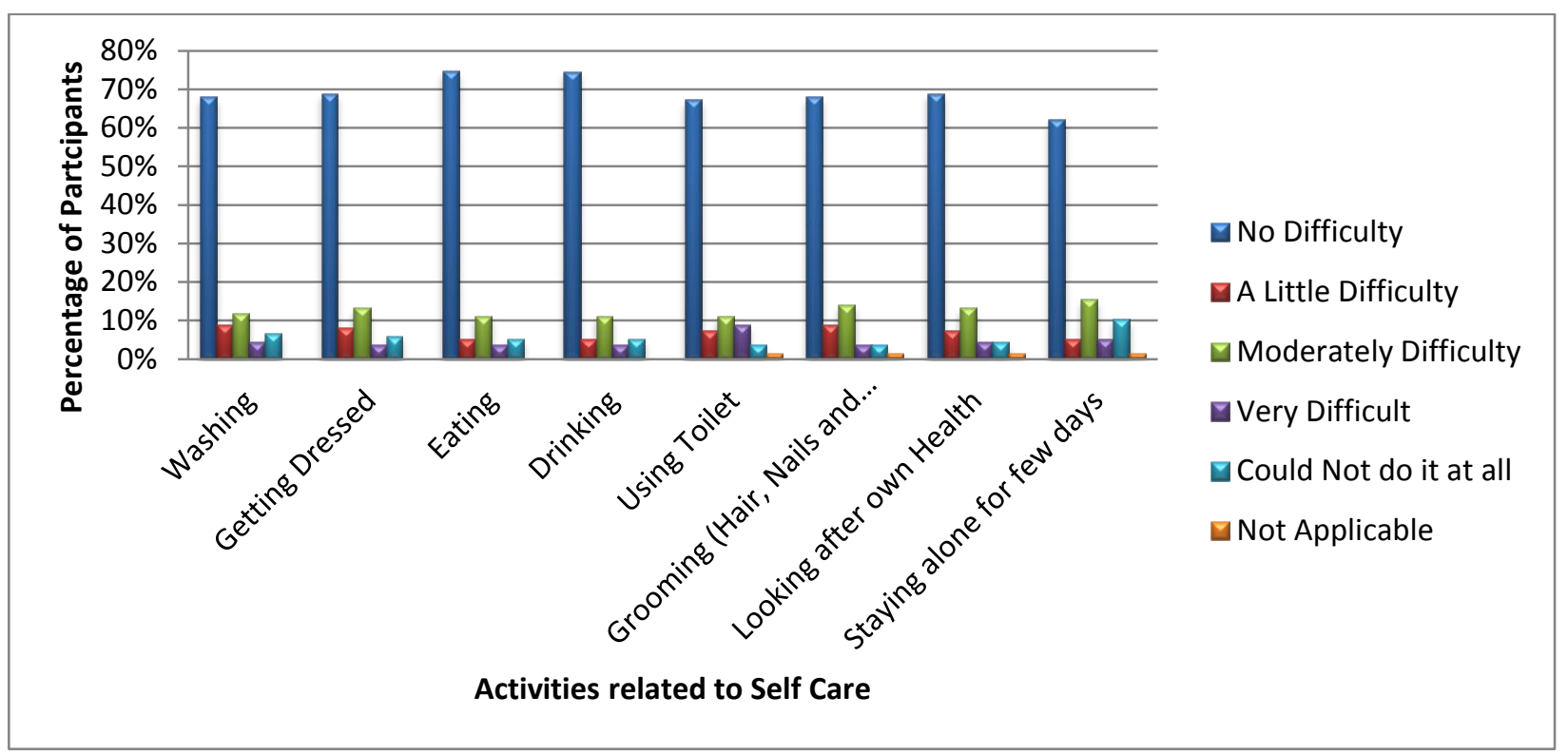

Figure 4.20 ICF Scores related to self-care 


\subsubsection{Mobility}

Figure 4.21 depicts ICF scores related to the mobility of participants using predominantly the upper limbs. Activities in this category presented participants with even more challenges. Lifting and carrying objects and driving a form of transportation were especially challenging.

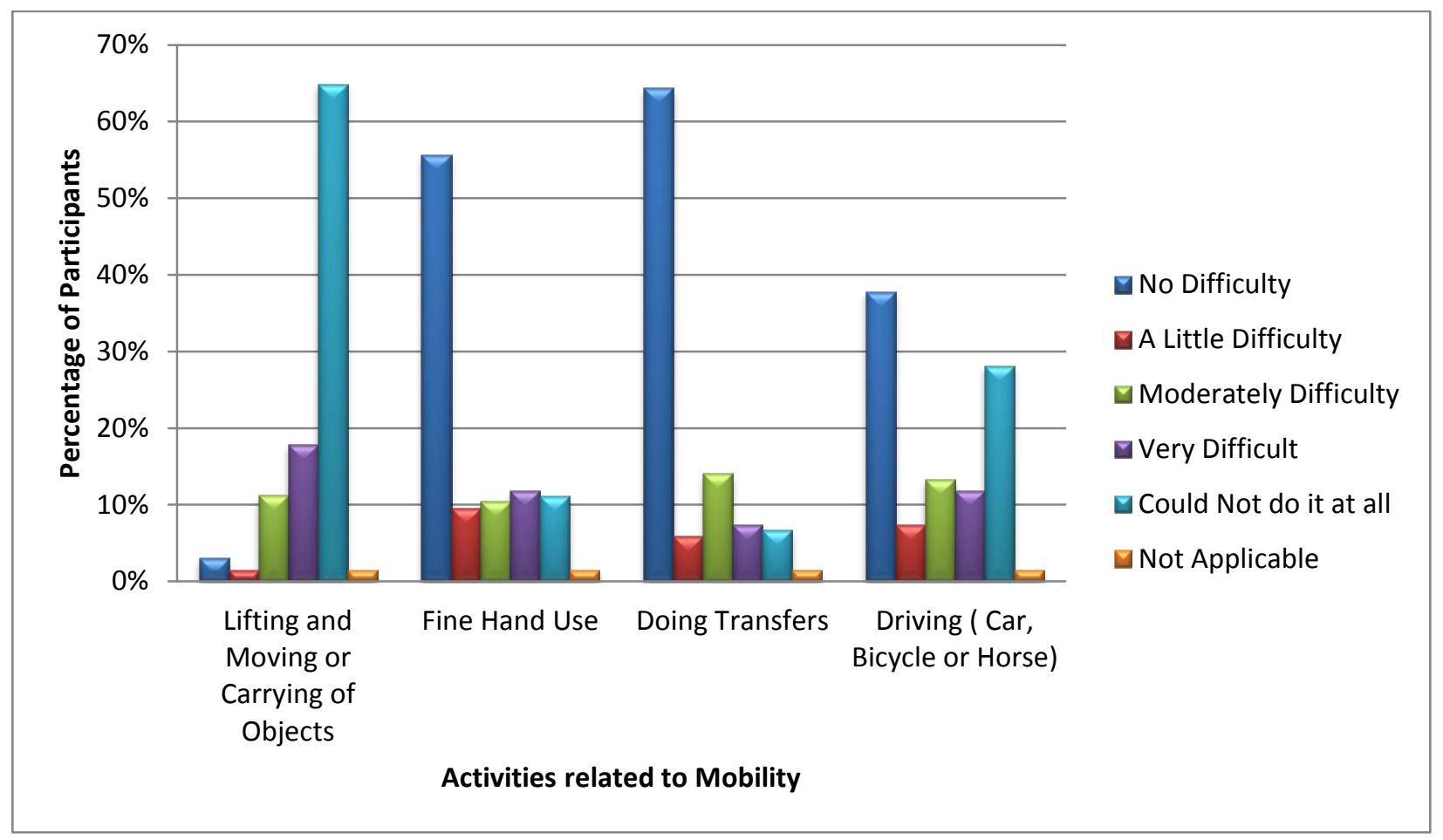

Figure 4.21 ICF scores related to mobility of the upper limbs 
Figure 4.22 summarises the ICF scores related to mobility using the lower limbs or an assistive device. The majority of participants (75\%) could get around in a wheelchair or through using another device, but fewer (50\%) could use transport and $49 \%$ could not walk at all.

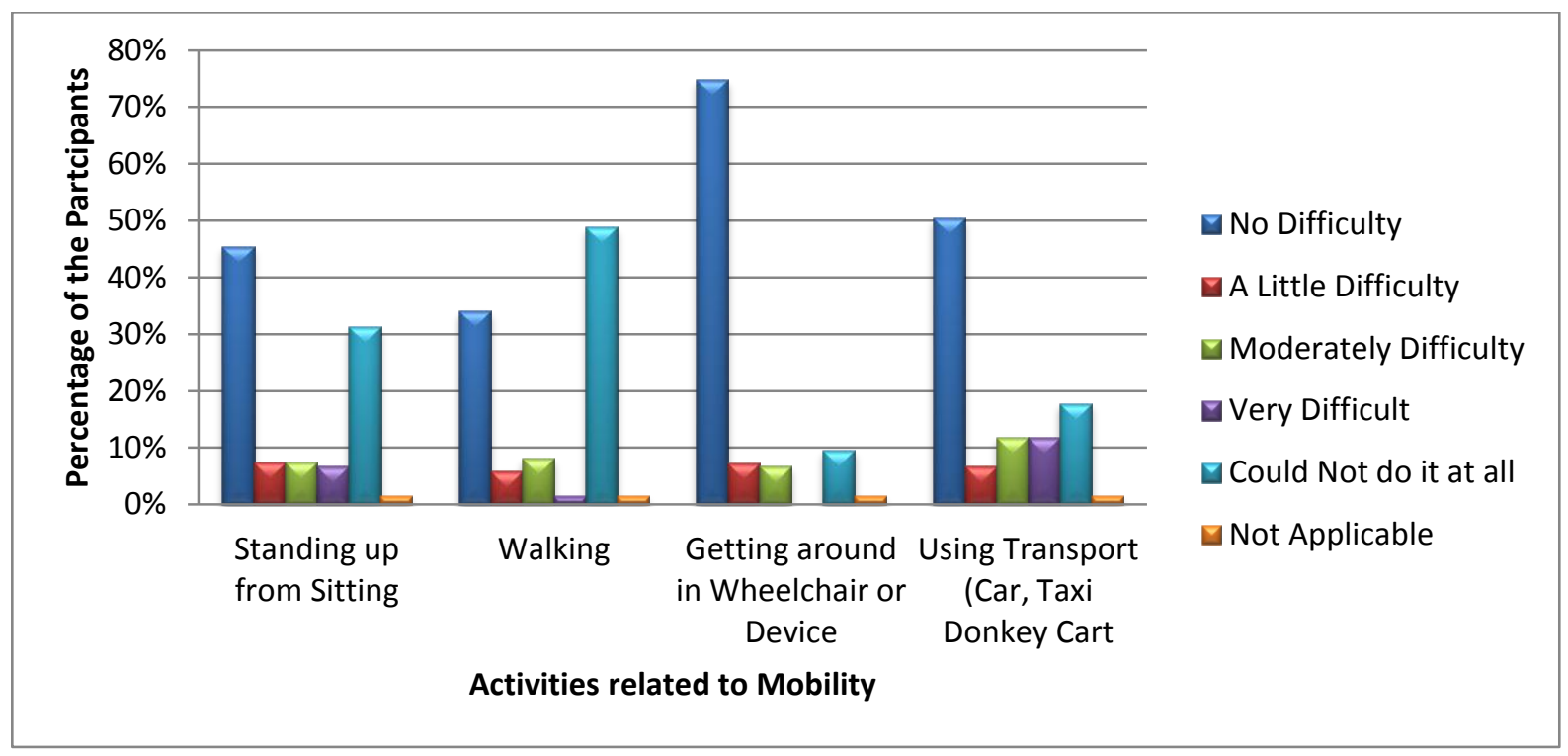

Figure 4.22 ICF scores related to mobility of the lower limbs 
Figure 4.23 show that outdoor mobility presented $77 \%$ of participants with no problems.

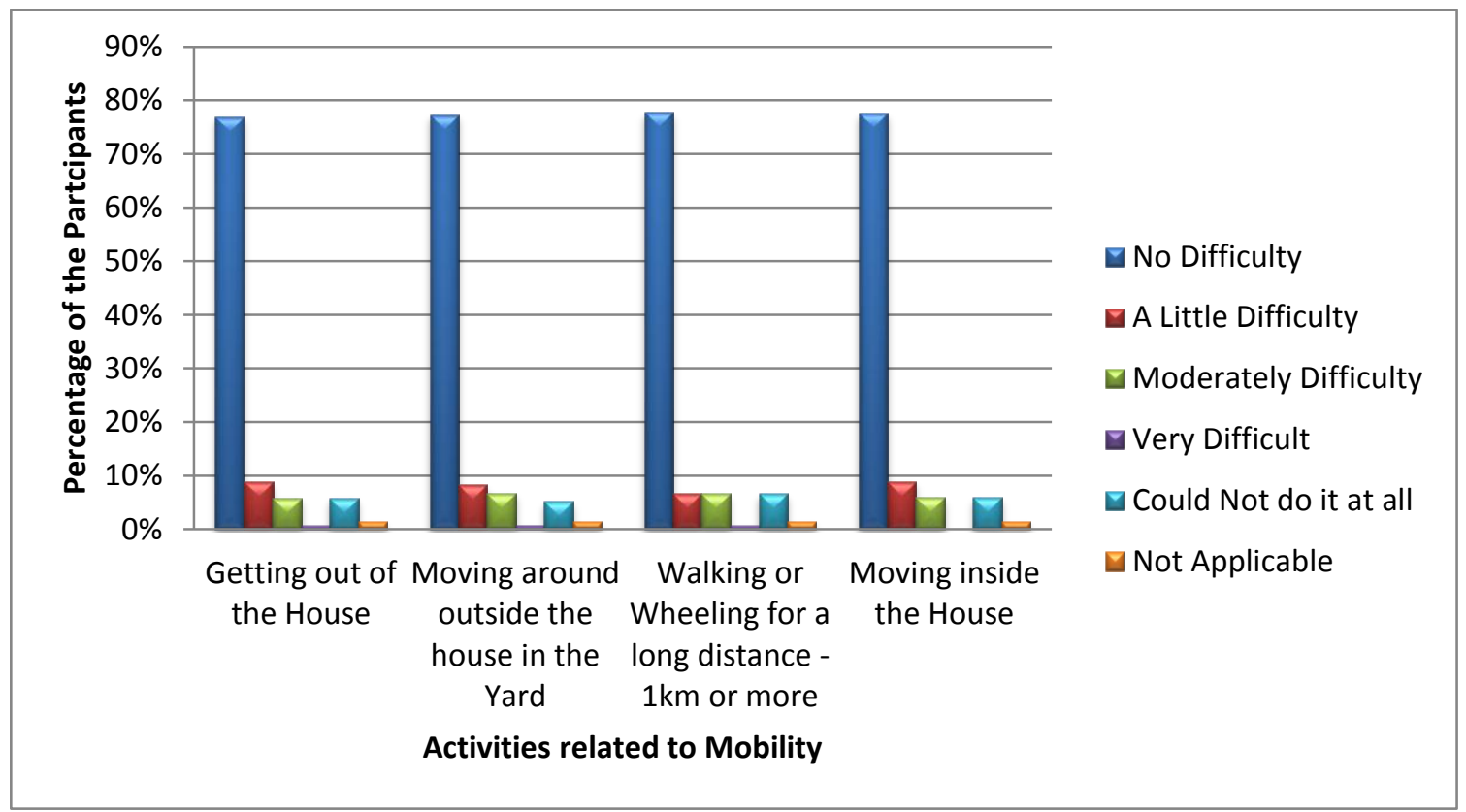

Figure 4.23 ICF scores related to mobility around the house 


\subsubsection{Domestic life}

Figure 4.24 showed that about half of participants experienced little or no challenges with regard to the performance of domestic activities, while the other half experienced moderate to severe challenges in this regard or could not do it at all.

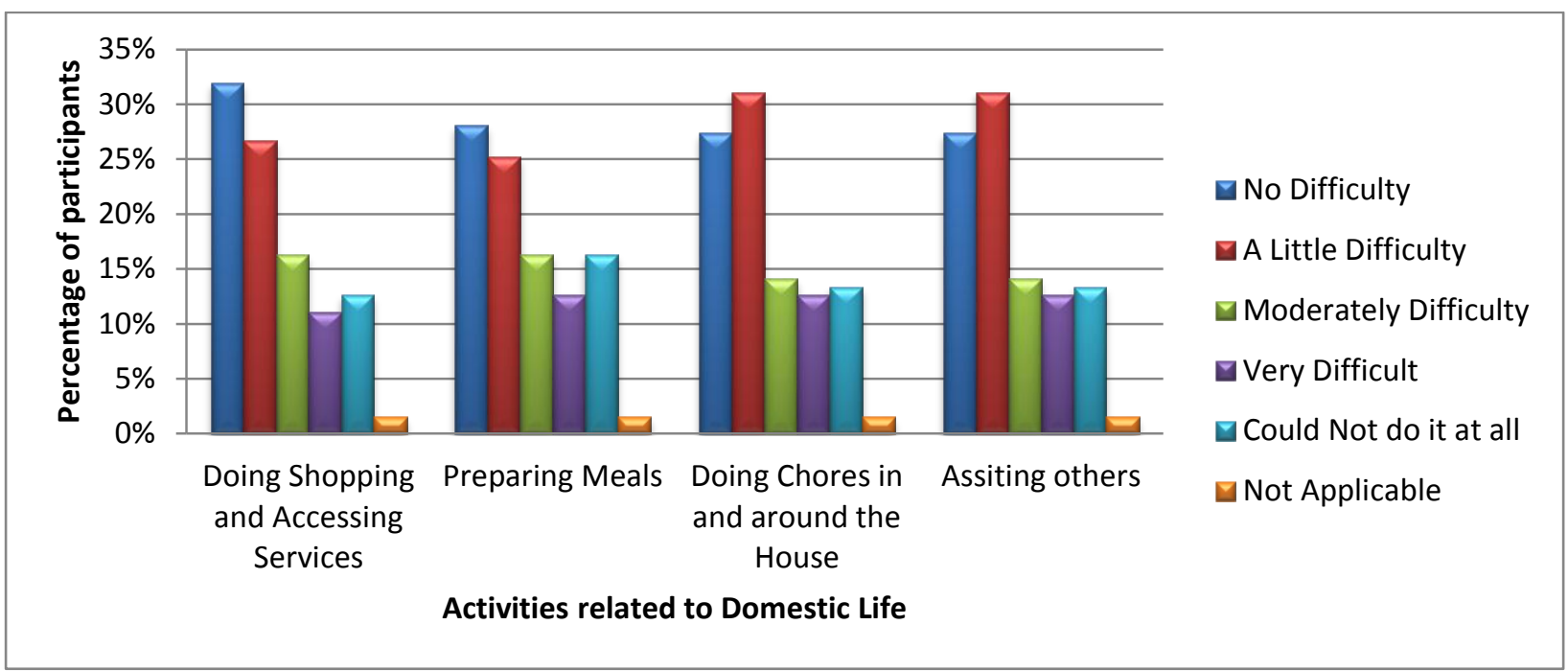

Figure 4.24 ICF scores related to domestic life 


\subsubsection{Community integration}

According to Figure 4.25 more than $60 \%$ of participants experienced no problems with community integration and participating in social, religious and sport activities, managing their own finances and participating in politics.

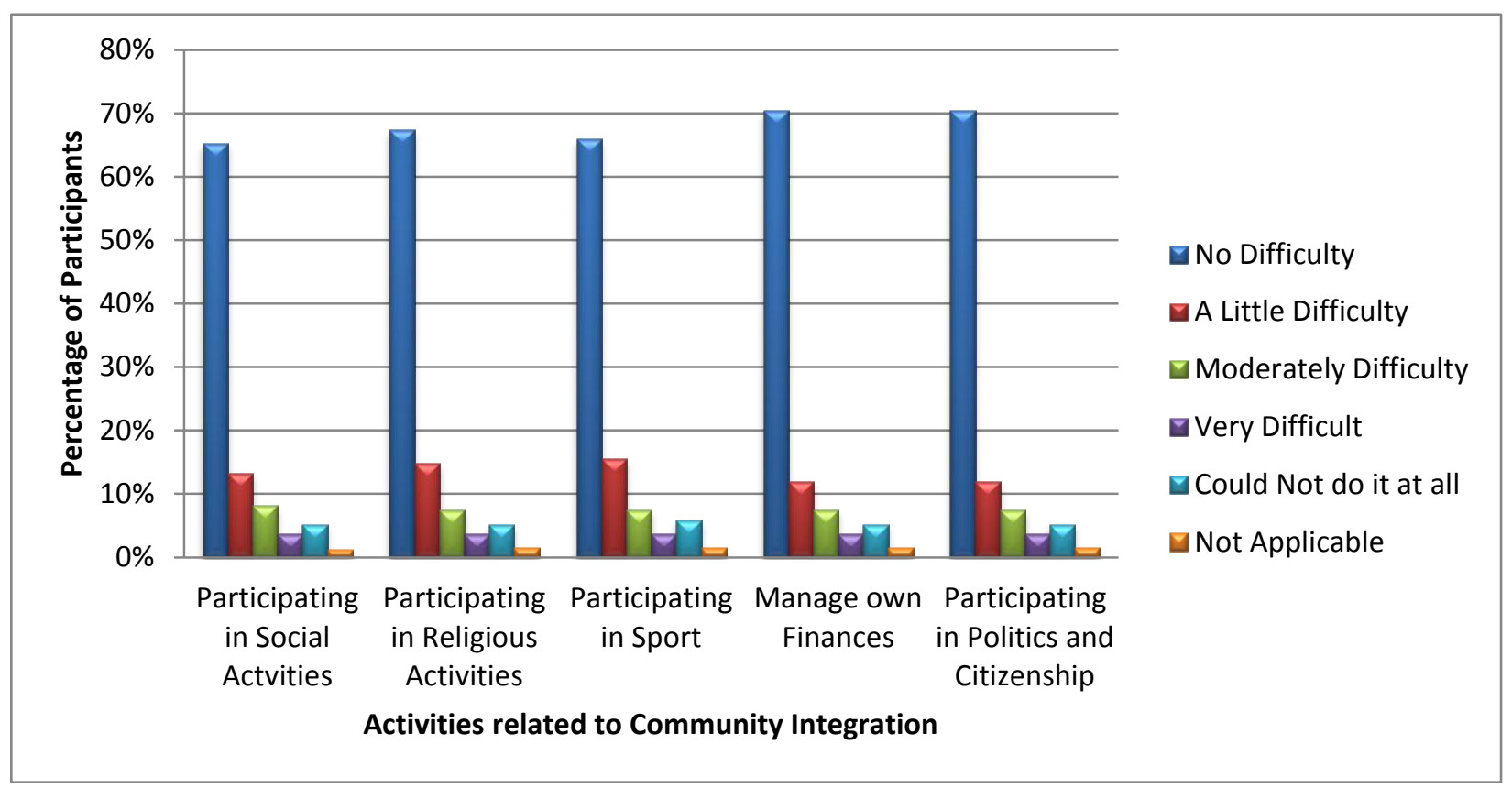

Figure 4.25 ICF Scores related to community integration 


\subsubsection{Productive activity}

Figure 4.26 indicates that $55 \%$ of participants were re-employed while $34 \%$ could not return to meaningful employment and $23 \%$ of participants were not economically self-sufficient.

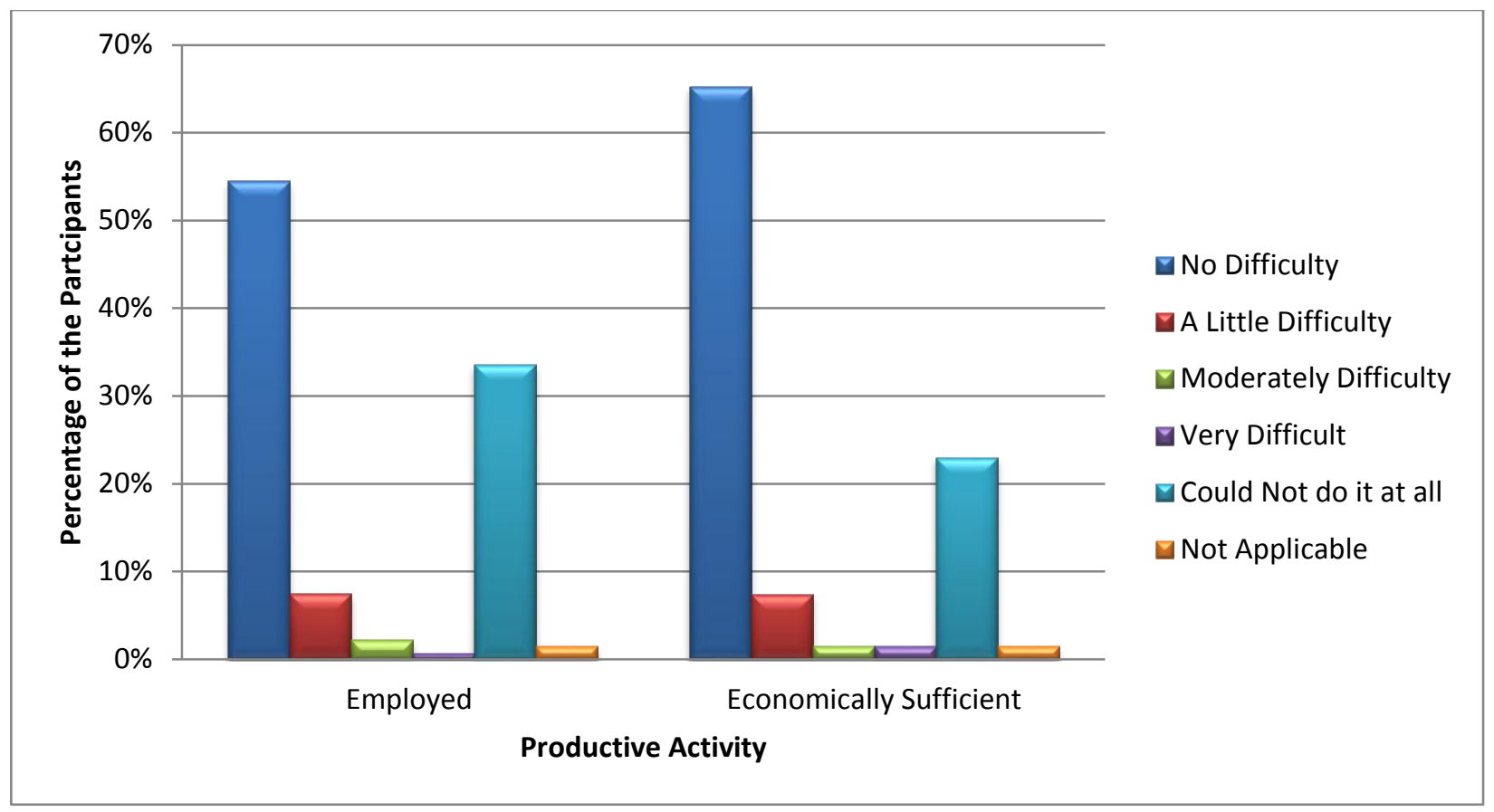

Figure 4.26 ICF Scores related to productive activity 
Table 4.13 compares the participant's employment status at discharge to the neurological level of the injury. For one participant data on re-employment was not available. The majority of participants with injuries to the cervical region (47\%) were re-employed by their previous employer. The majority of participants with thoracic injuries (44\%) were unemployed at time of discharge. Participants with injuries to the lumbar $(63 \%)$ region of the spine mostly returned to their previous employer. One third of participants were unemployed at time of discharge. In total, $49 \%$ of participants with cervical injuries, $49 \%$ of participants with thoracic lesions and $69 \%$ of participants with lumbar injuries were re-employed. The Fisher Exact Test showed a statistically significant relationship between the neurological levels (in total) and the employment status at discharge $(\mathrm{p}=0.036)$.

Table 4.13 Employment status and neurological level

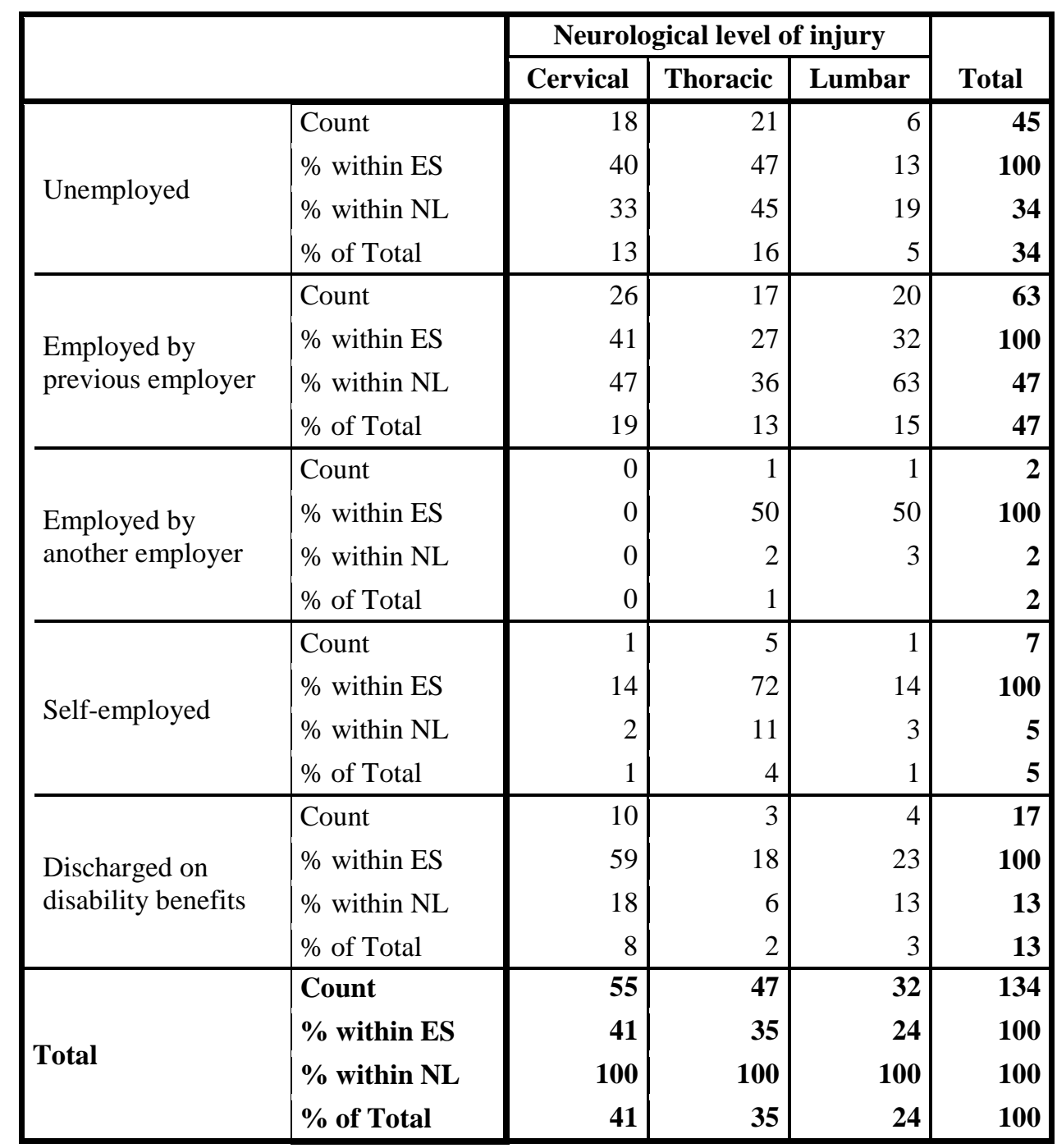


Table 4.14 shows that participants suffering from injuries classified as ASIA A (42\%) were most often unemployed. Participants with ASIA B (47\%), ASIA C (36\%) and ASIA D (38\%) were most often employed by their previous employer. The Fisher Exact Test revealed no statistically significant relationship between the employment status and the classification of injury $(\mathrm{p}=0.76)$.

Table 4.14 Employment status at discharge and classification of injury

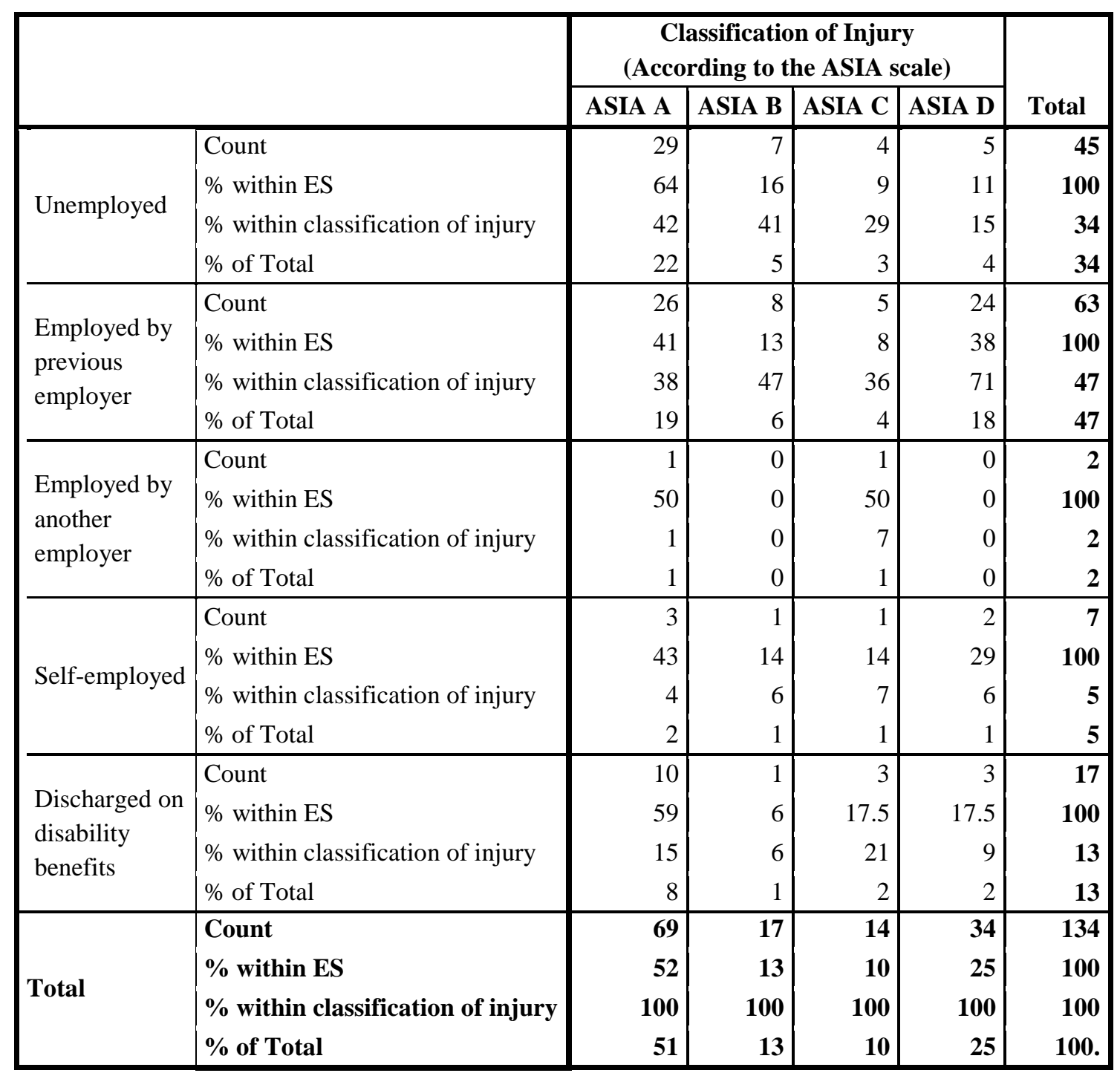


Table 4.15 (over two pages) summarises information on the employment status of participants at discharge and the type of industry they were employed in. Amongst the unemployed (34\%), 24\% were from the service industry, $16 \%$ from the construction industry and $0 \%$ from the manufacturing industry. Forty seven per cent of participants were employed by their previous employer. Out of these, $41 \%$ were in the service industry, $19 \%$ in the mining industry, $16 \%$ from engineering and $3 \%$ from the manufacturing and transport industries. Thirteen per cent of participants were discharged from active employment with disability benefits. Of these, $47 \%$ were from the service industry and $18 \%$ in the engineering sector. The mining industry re-employed $63 \%$ of employees, the service industry reemployed $55 \%$ and the engineering industry $50 \%$ of their employees.

Table 4.15 Employment status and type of industry

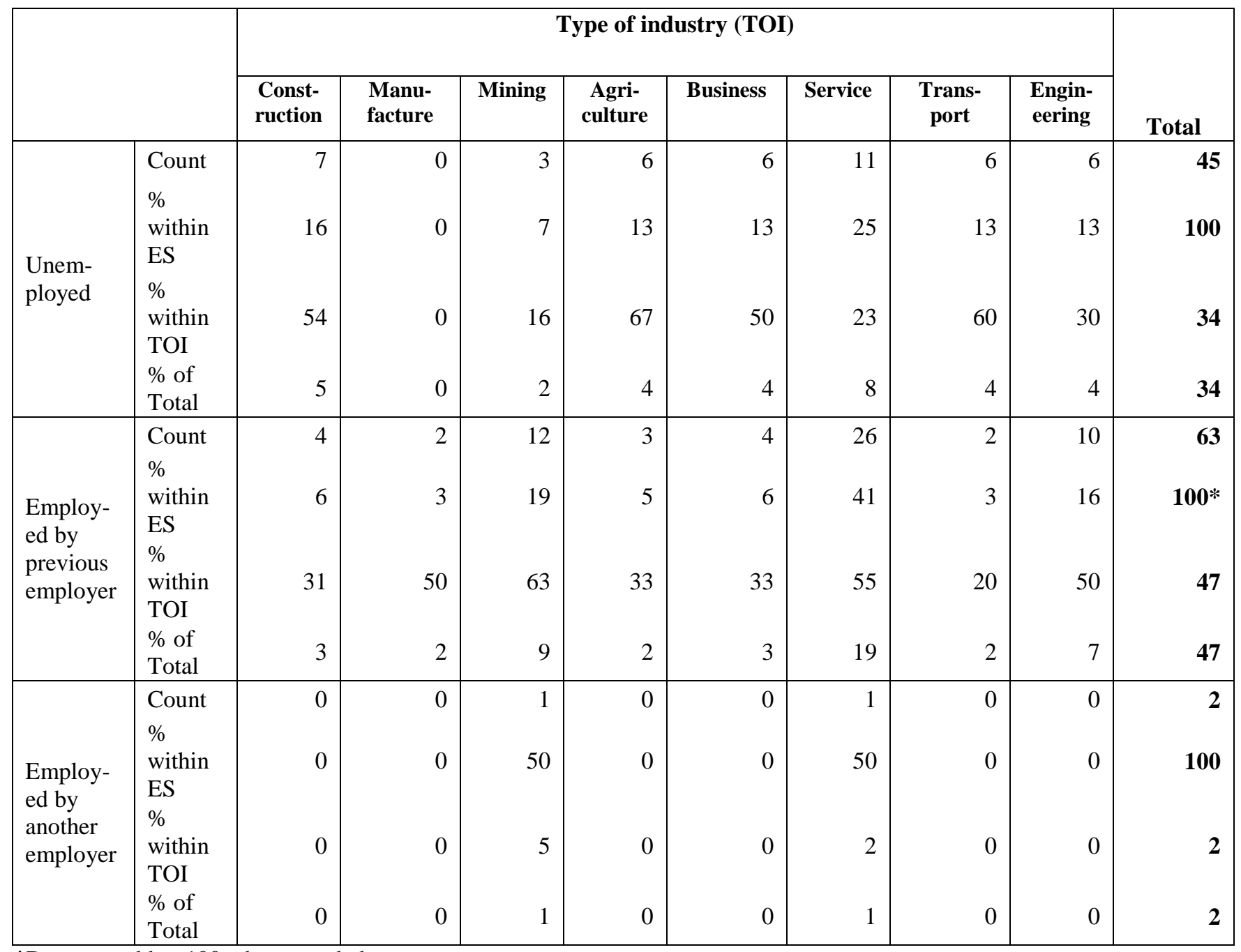

*Does not add to 100 when rounded 
Table 4.15 Employment status and type of industry (continued)

\begin{tabular}{|c|c|c|c|c|c|c|c|c|c|c|}
\hline & \multicolumn{8}{|c|}{ Type of industry (TOI) } & \multirow[b]{2}{*}{ Total } \\
\hline & & $\begin{array}{l}\text { Const- } \\
\text { ruction }\end{array}$ & $\begin{array}{l}\text { Manu- } \\
\text { facture }\end{array}$ & Mining & $\begin{array}{c}\text { Agri- } \\
\text { culture }\end{array}$ & Business & Service & $\begin{array}{c}\text { Trans- } \\
\text { port }\end{array}$ & $\begin{array}{l}\text { Engin- } \\
\text { eering }\end{array}$ & \\
\hline \multirow{5}{*}{$\begin{array}{l}\text { Self } \\
\text { employed }\end{array}$} & Count & 2 & 0 & 1 & 0 & 2 & 1 & 0 & 1 & 7 \\
\hline & within & 29 & 0 & 14 & 0 & 29 & 14 & 0 & 14 & 100.00 \\
\hline & $\%$ & & & & & & & & & \\
\hline & $\begin{array}{l}\text { within } \\
\text { TOI }\end{array}$ & 15 & 0 & 5 & 0 & 17 & 2 & 0 & 5 & 5 \\
\hline & $\begin{array}{l}\% \text { of } \\
\text { Total }\end{array}$ & 1 & 0 & 1 & 0 & 1 & 1 & 0 & 1 & 5 \\
\hline \multirow{5}{*}{$\begin{array}{l}\text { Dischar- } \\
\text { ged on } \\
\text { disability } \\
\text { benefits }\end{array}$} & Count & 0 & 2 & 2 & 0 & 0 & 8 & 2 & 3 & 17 \\
\hline & $\begin{array}{l}\% \\
\text { within }\end{array}$ & 0 & 12 & 12 & 0 & 0 & 47 & 12 & 17 & 100 \\
\hline & $\begin{array}{l}\text { ES } \\
\%\end{array}$ & & & & & & & & & \\
\hline & $\begin{array}{l}\text { within } \\
\text { TOI }\end{array}$ & 0 & 50 & 10.50 & 0.00 & 0.00 & 17.00 & 20.00 & 15.00 & 13 \\
\hline & $\begin{array}{l}\% \text { of } \\
\text { Total }\end{array}$ & 0 & 1 & 1 & 0 & 0 & 6 & 1 & 2 & 13 \\
\hline \multirow{5}{*}{ Total } & Count & 13 & 4 & 19 & 9 & 12 & 47 & 10 & 20 & 134 \\
\hline & $\begin{array}{l}\% \\
\text { within }\end{array}$ & 10 & 3 & 14 & 7 & 9 & 35 & 7 & 15 & 100 \\
\hline & $\begin{array}{l}\text { ES } \\
\%\end{array}$ & & & & & & & & & \\
\hline & within & 100 & 100 & 100 & 100 & 100 & 100 & 100 & 100 & 100 \\
\hline & $\begin{array}{l}\% \text { of } \\
\text { Total }\end{array}$ & 10 & 3 & 14 & 7 & 9 & 35 & 7 & 15 & 100 \\
\hline
\end{tabular}


Table 4.16 shows that for the $34 \%$ of participants who were unemployed at the time of discharge the most commonly affected occupations were labourers (38\%) and professionals (18\%). The least affected were police officers $(2 \%)$. Those that were employed by their previous employers most frequently were professionals (30\%), labourers (22\%) and police officers (21\%).

Table 4.16 Employment Status and Occupation

\begin{tabular}{|c|c|c|c|c|c|c|c|c|c|}
\hline & \multicolumn{7}{|c|}{ Occupation } & \multirow[b]{2}{*}{ Total } \\
\hline & & $\begin{array}{l}\text { Security } \\
\text { Officer }\end{array}$ & $\begin{array}{l}\text { Police } \\
\text { Officer }\end{array}$ & Labourer & Miner & Driver & $\begin{array}{l}\text { Mana- } \\
\text { ger }\end{array}$ & $\begin{array}{c}\text { Profes- } \\
\text { sional }\end{array}$ & \\
\hline \multirow{4}{*}{ Unemployed } & Count & 7 & 1 & 17 & 3 & 7 & 2 & 8 & 45 \\
\hline & $\%$ within ES & 15 & 2 & 38 & 7 & 16 & 4 & 18 & 100 \\
\hline & $\begin{array}{l}\% \text { within } \\
\text { occupation }\end{array}$ & 58 & 6 & 49 & 21 & 44 & 22 & 25 & 34 \\
\hline & $\%$ of Total & 5 & 1 & 13 & 2 & 5 & 2 & 6 & 34 \\
\hline \multirow{4}{*}{$\begin{array}{l}\text { Employed by } \\
\text { previous } \\
\text { employer }\end{array}$} & Count & 1 & 13 & 14 & 8 & 3 & 5 & 19 & 63 \\
\hline & $\%$ within ES & 2 & 20 & 22 & 13 & 5 & 8 & 30 & 100 \\
\hline & $\begin{array}{l}\% \text { within } \\
\text { occupation }\end{array}$ & 8 & 81 & 40 & 57 & 19 & 56 & 59 & 47 \\
\hline & $\%$ of Total & 1 & 10 & 10 & 6 & 2 & 4 & 14 & 47 \\
\hline \multirow{4}{*}{$\begin{array}{l}\text { Employed by } \\
\text { another } \\
\text { employer }\end{array}$} & Count & 1 & 0 & 0 & 1 & 0 & 0 & 0 & 2 \\
\hline & $\%$ within ES & 50 & 0 & 0 & 50 & 0 & 0 & 0 & 100 \\
\hline & $\begin{array}{l}\% \text { within } \\
\text { occupation }\end{array}$ & 8 & 0 & 0 & 7 & 0 & 0 & 0 & 2 \\
\hline & $\%$ of Total & 1 & 0 & 0 & 1 & 0 & 0 & 0 & 2 \\
\hline \multirow{4}{*}{$\begin{array}{l}\text { Self } \\
\text { employed }\end{array}$} & Count & 0 & 0 & 1 & 1 & 0 & 0 & 5 & 7 \\
\hline & $\%$ within ES & 0 & 0 & 14 & 14 & 0 & 0 & 72 & 100 \\
\hline & $\begin{array}{l}\% \text { within } \\
\text { occupation }\end{array}$ & 0 & 0 & 3 & 7 & 0 & 0 & 16 & 5 \\
\hline & $\%$ of Total & 0 & 0 & 1 & 1 & 0 & 0 & 4 & 5 \\
\hline \multirow{4}{*}{$\begin{array}{l}\text { Discharged } \\
\text { on Disability } \\
\text { Benefits }\end{array}$} & Count & 3 & 2 & 3 & 1 & 6 & 2 & 0 & 17 \\
\hline & $\%$ within ES & 18 & 12 & 18 & 6 & 35 & 12 & 0 & $* 100$ \\
\hline & $\begin{array}{l}\% \text { within } \\
\text { occupation }\end{array}$ & 25 & 13 & 9 & 7 & 37 & 22 & 0 & 13 \\
\hline & $\%$ of Total & 2 & 1 & 2 & 1 & 5 & 1 & 0 & 13 \\
\hline \multirow{4}{*}{ Total } & Count & 12 & 16 & 35 & 14 & 16 & 9 & 32 & 134 \\
\hline & $\begin{array}{l}\% \text { within } \\
\text { employment } \\
\text { status }\end{array}$ & 9 & 12 & 26 & 10 & 12 & 7 & 24 & 100 \\
\hline & $\begin{array}{l}\% \text { within } \\
\text { occupation }\end{array}$ & 100 & 100 & 100 & 100 & 100 & 100 & 100 & 100 \\
\hline & $\%$ of Total & 9 & 12 & 26 & 10 & 12 & 7 & 24 & 100 \\
\hline
\end{tabular}

*Does not add to 100 when rounded 
Table 4.17 summarises the employment status and the population group. The Fisher Exact Test revealed no statistically significant relationship between employment status and population group $(\mathrm{p}=0.62)$.

Table 4.17 Employment status (ES) and population group (PG)

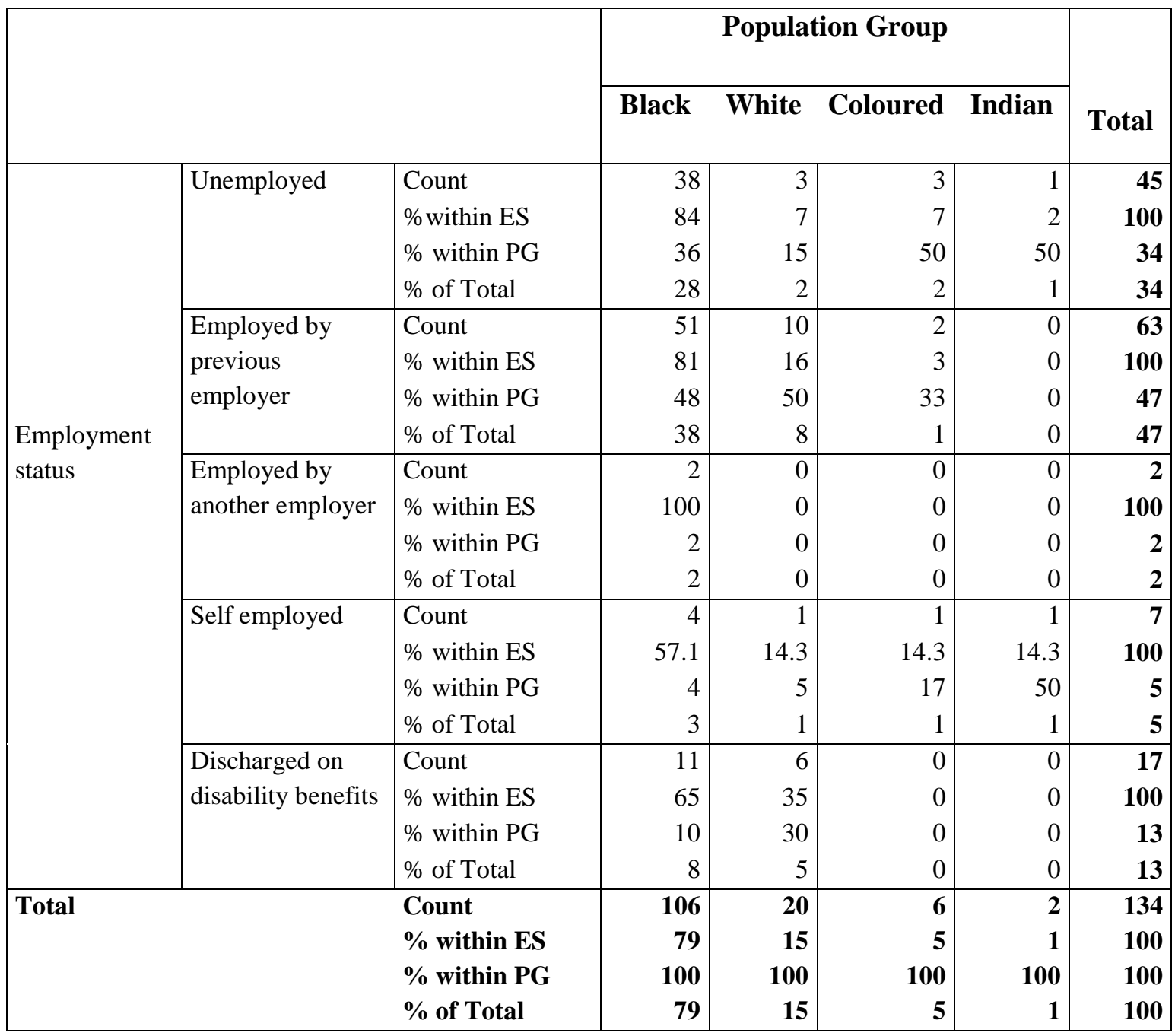


Table 4.18 summarises the employment status on discharge and the geographical location of participants. The majority of participants who were unemployed were in the urban (49\%) and periurban $(38 \%)$ areas. Participants who were employed by previous employer were from peri-urban (49\%) and urban areas (40\%). All participants that were self-employed were in the urban area.

The majority of participants that were discharged with disability benefits were from the urban area (65\%). Re-employment status at discharge differs very little between the three areas with $50 \%$ of those living and working in urban areas being re-employed and $60 \%$ of those in both peri-urban and rural areas being re-employed. Using the Fishers Exact Test showed geographical location had no statistically significant impact on employment status $(\mathrm{p}=0.109)$.

Table 4.18 Geographical locations and employment status

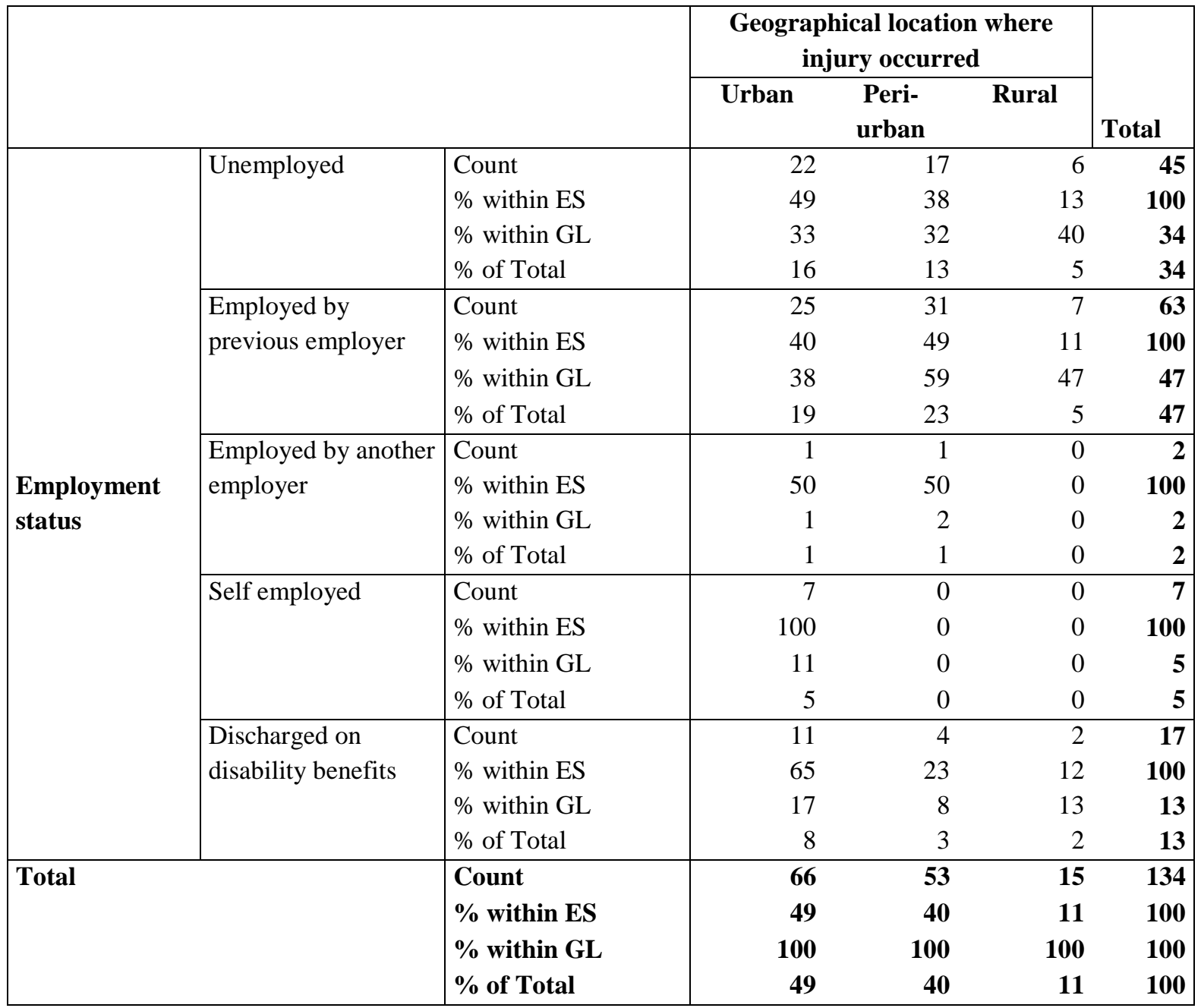




\subsubsection{FIM+FAM scores on admission and discharge}

The outcome measures used to assess the functional outcomes of rehabilitation following SCI in the two facilities was the FIM and FAM. The scores were taken on admission and discharge in the facility. Table 4.19 summarises the descriptive statistics of the FIM+FAM scores on admission (both cognitive and functional) and on discharge.

Table 4.19 Descriptive statistics on FIM+FAM scores on admission and discharge

\begin{tabular}{|l|r|r|r|r|r|r|r|}
\hline & N & Range & Minimum & Maximum & Mean & $\begin{array}{c}\text { Std. } \\
\text { Error }\end{array}$ & $\begin{array}{c}\text { Std. } \\
\text { Deviation }\end{array}$ \\
\hline $\begin{array}{l}\text { Cognitive FIM scores } \\
\text { (on admission) }\end{array}$ & 135 & 84 & 14 & 98 & 80.30 & 1.379 & 16.019 \\
$\begin{array}{l}\text { Functional FIM+FAM } \\
\text { Scores (on admission) }\end{array}$ & 135 & 85 & 16 & 101 & 46.74 & 1.777 & 20.649 \\
$\begin{array}{l}\text { Functional FIM scores } \\
\text { (on discharge) }\end{array}$ & 135 & 94 & 16 & 110 & 79.36 & 2.173 & 25.243 \\
$\begin{array}{l}\text { Cognitive FIM+FAM } \\
\text { score (on discharge) }\end{array}$ & 135 & 84 & 14 & 98 & 87.63 & 1.077 & 12.510 \\
Valid N & 135 & & & & & & \\
\hline
\end{tabular}

As shown in Table 4.19 a mean gain in functional FIM+FAM scores of 32.62 was made between admission and discharge. A Wilcoxon Signed Ranks Test was used to determine if any statistical relationship existed between FIM+FAM scores on admission and discharge per neurological level. All regions of the spine for both cognitive and functional FIM+FAM scores showed statistically significant gains ( $\mathrm{p}$-value $=0.000$ ). 


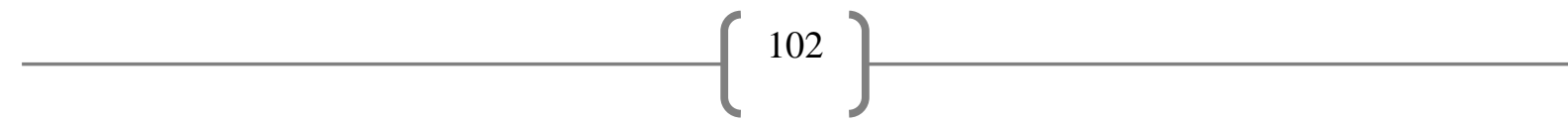

Tables 4.20 and 4.21 summarise the descriptive statistics of the functional and cognitive FIM+FAM scores on admission and discharge per neurological level. The mean functional discharge scores were almost double those of the mean admission scores, while much smaller gains were made in cognitive scores.

Table 4.20 Descriptive statistics on functional FIM+FAM scores per neurological level (NL)

\begin{tabular}{|c|c|c|c|}
\hline NL & & $\begin{array}{c}\text { Functional } \\
\text { FIM+FAM } \\
\text { score on } \\
\text { admission }\end{array}$ & $\begin{array}{c}\text { Functional } \\
\text { FIM+FAM } \\
\text { score on } \\
\text { discharge }\end{array}$ \\
\hline $\begin{array}{l}\text { Cervical } \\
(\mathrm{N}=56)\end{array}$ & $\begin{array}{l}\text { Mean } \\
\text { Std. Deviation } \\
\text { Minimum } \\
\text { Maximum }\end{array}$ & $\begin{array}{r}39.93 \\
20.762 \\
16 \\
96\end{array}$ & $\begin{array}{r}67.41 \\
28.866 \\
16 \\
110\end{array}$ \\
\hline $\begin{array}{l}\text { Thoracic } \\
(\mathrm{N}=47)\end{array}$ & $\begin{array}{l}\text { Mean } \\
\text { Std. Deviation } \\
\text { Minimum } \\
\text { Maximum }\end{array}$ & $\begin{array}{r}48.49 \\
19.211 \\
18 \\
101\end{array}$ & $\begin{array}{r}82.83 \\
18.518 \\
23 \\
106\end{array}$ \\
\hline $\begin{array}{l}\text { Lumbar } \\
(\mathrm{N}=32)\end{array}$ & $\begin{array}{l}\text { Mean } \\
\text { Std. Deviation } \\
\text { Minimum } \\
\text { Maximum }\end{array}$ & $\begin{array}{r}55.84 \\
18.813 \\
30 \\
100\end{array}$ & $\begin{array}{r}95.16 \\
15.278 \\
34 \\
110\end{array}$ \\
\hline $\begin{array}{l}\text { Total } \\
(\mathrm{N}=135)\end{array}$ & $\begin{array}{l}\text { Mean } \\
\text { Std. Deviation } \\
\text { Minimum } \\
\text { Maximum }\end{array}$ & $\begin{array}{r}46.68 \\
20.634 \\
16 \\
101\end{array}$ & $\begin{array}{r}79.36 \\
25.243 \\
16 \\
110\end{array}$ \\
\hline
\end{tabular}




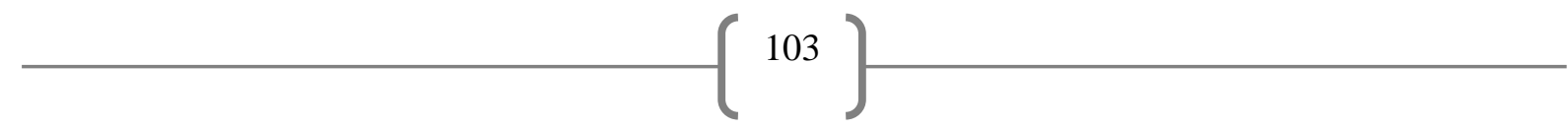

Table 4.21 Descriptive statistics on cognitive FIM+FAM scores per neurological level (NL)

\begin{tabular}{|c|c|c|c|}
\hline NL & & $\begin{array}{l}\text { Cognitive } \\
\text { score on } \\
\text { admission }\end{array}$ & $\begin{array}{c}\text { Cognitive } \\
\text { score on } \\
\text { discharge }\end{array}$ \\
\hline $\begin{array}{l}\text { Cervical } \\
(\mathrm{N}=56)\end{array}$ & $\begin{array}{l}\text { Mean } \\
\text { Std. Deviation } \\
\text { Minimum } \\
\text { Maximum }\end{array}$ & $\begin{array}{r}74.21 \\
17.109 \\
14 \\
98\end{array}$ & $\begin{array}{r}82.82 \\
15.507 \\
14 \\
98\end{array}$ \\
\hline $\begin{array}{l}\text { Thoracic } \\
(\mathrm{N}=47)\end{array}$ & $\begin{array}{l}\text { Mean } \\
\text { Std. Deviation } \\
\text { Minimum } \\
\text { Maximum } \\
\end{array}$ & $\begin{array}{r}84.06 \\
14.510 \\
29 \\
98 \\
\end{array}$ & $\begin{array}{r}90.36 \\
9.023 \\
58 \\
98 \\
\end{array}$ \\
\hline $\begin{array}{l}\text { Lumbar } \\
(\mathrm{N}=32)\end{array}$ & $\begin{array}{l}\text { Mean } \\
\text { Std. Deviation } \\
\text { Minimum } \\
\text { Maximum }\end{array}$ & $\begin{array}{r}85.41 \\
12.735 \\
59 \\
98\end{array}$ & $\begin{array}{r}92.03 \\
7.481 \\
69 \\
98\end{array}$ \\
\hline $\begin{array}{l}\text { Total } \\
(\mathrm{N}=135)\end{array}$ & $\begin{array}{l}\text { Mean } \\
\text { Std. Deviation } \\
\text { Minimum } \\
\text { Maximum }\end{array}$ & $\begin{array}{r}80.30 \\
16.019 \\
14 \\
98\end{array}$ & $\begin{array}{r}87.63 \\
12.510 \\
14 \\
98\end{array}$ \\
\hline
\end{tabular}




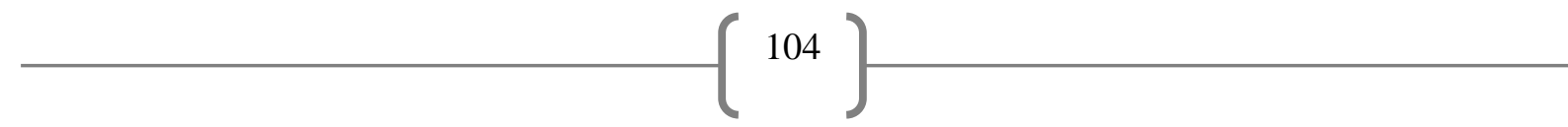

Table 4.22 summarises the individual differences of the FIM+FAM means scores (on admission and discharge) of participants who sustained SCI in the cervical region. $25 \%$ of participants with cervical lesions gained up to 14 points in their functional abilities following rehabilitation. Seventy-five percent of participants in this category had up to 40.75 points gain while $50 \%$ had gained up to 25.5 points. There were marginal gains in the cognitive aspects of the FIM+FAM scores with only up to 14 points gain in $75 \%$ of participants.

Table 4.22 Individual differences (diff) in FIM+FAM and FAM scores (cervical region of the spine)

\begin{tabular}{|c|c|c|c|}
\hline & & Function (diff) & Cognitive (diff) \\
\hline \multicolumn{2}{|l|}{$\mathrm{N}$} & 56 & 56 \\
\hline \multicolumn{2}{|l|}{ Median } & 25.5000 & 5.5000 \\
\hline \multicolumn{2}{|l|}{ Mode } & $.00^{\mathrm{a}}$ & .00 \\
\hline \multicolumn{2}{|l|}{ Minimum } & .00 & -16.00 \\
\hline \multicolumn{2}{|l|}{ Maximum } & 66.00 & 41.00 \\
\hline \multirow[t]{3}{*}{ Percentiles } & 25 & 14.0000 & 1.0000 \\
\hline & 50 & 25.5000 & 5.5000 \\
\hline & 75 & 40.7500 & 15.0000 \\
\hline
\end{tabular}

a. Multiple modes exist. The smallest value is shown

Table 4.23 summarises the individual differences of the FIM+FAM means scores (on admission and discharge) of participants who sustained SCIs in the thoracic region. Twenty-five percent of participants had up to 24 points gain in functional aspect, $50 \%$ of participants had up to 38 points gain and $75 \%$ of participants had up to 48 points gain. Again, the cognitive scores gain were marginal but with statistical significance ( $\mathrm{p}$-value=0.00). Seventy-five percent of participants had up to 10 points gain in cognitive scores.

Table 4.23 Individual difference (diff) in FIM+FAM scores (thoracic region of the spine)

\begin{tabular}{|ll|r|r|}
\hline & Function (diff) & Cognitive (diff) \\
\hline $\mathrm{N}$ & 47 & 47 \\
Median & & 38.0000 & 4.0000 \\
Mode & 24.00 & .00 \\
Minimum & .00 & .00 \\
Maximum & & 63.00 & 30.00 \\
Percentiles & 25 & 24.0000 & .0000 \\
& 50 & 38.0000 & 4.0000 \\
& 75 & 48.0000 & 10.0000 \\
\hline
\end{tabular}




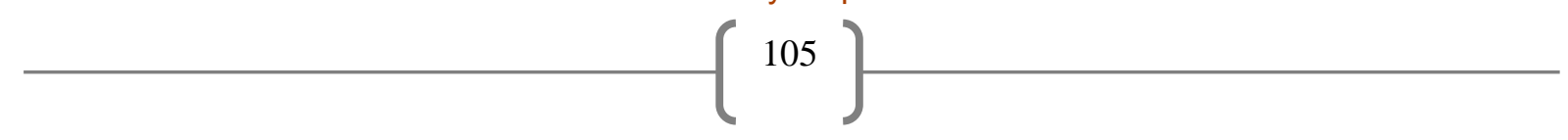

Table 4.24 summarises the individual differences of the FIM+FAM means scores (on admission and discharge) of participants who sustained SCIs in the lumbar region. Twenty-five percent of participants had up to 27 points gain in functional scores, $50 \%$ of participants had up to 40.5 points gain and $75 \%$ of participants had up to 52.75 points gain. Again, cognitive score gains are marginal although statistically significant ( $p$-value $=0.00$ ). Seventy-five percent of participants had up to 11 points gain in cognitive scores.

Table 4.24 Individual differences (diff) in FIM+FAM scores (lumbar region of the spine)

\begin{tabular}{|c|c|c|c|}
\hline & & Function (diff) & Cognitive (diff) \\
\hline \multicolumn{2}{|l|}{$\mathrm{N}$} & 32 & 32 \\
\hline \multicolumn{2}{|l|}{ Median } & 40.5000 & 5.0000 \\
\hline \multicolumn{2}{|l|}{ Mode } & 66.00 & .00 \\
\hline \multicolumn{2}{|l|}{ Minimum } & 4.00 & .00 \\
\hline \multicolumn{2}{|l|}{ Maximum } & 68.00 & 31.00 \\
\hline \multirow[t]{3}{*}{ Percentiles } & 25 & 27.0000 & .0000 \\
\hline & 50 & 40.5000 & 5.0000 \\
\hline & 75 & 52.7500 & 11.0000 \\
\hline
\end{tabular}




\subsection{Chapter Summary}

Males in the 26-35 age range were the most affected by work-related SCIs in this study. The majority of participants were married. Most of the injuries occurred in urban areas and blacks were mostly affected. The majority of participants were employed in the service industry and the most affected employees were labourers. The major cause of work-related SCI was motor vehicle accidents. The majority (58.4\%) of the participants were classified as paraplegic and $51 \%$ of the participants' injuries were classified as complete. The average annual admission rate of individuals with work-related SCIs at both research settings was 14 . The injuries mostly occurred in the afternoon, on Fridays, and during the months of February and April. The most commonly affected region of the spine was the cervical spine. The majority of participants had complete lesions and from those with incomplete lesions the majority were classified as ASIA D.

The average admission rate of individuals with work-related SCIs at both research settings was 13.5(14) people per year. Most participants received medical rehabilitation, cognitive behavioural therapy, diet therapy and counselling. The majority of participants did not receive vocational rehabilitation. The most commonly issued assistive devices were wheelchairs and wheelchair cushions.

Participants with upper cervical lesions stayed longer for rehabilitation. Participants who stayed longer in the rehabilitation facility had improved functional outcomes. Participants who sustained cervical lesions were more expensive to rehabilitate than those with thoracic and lumbar injuries.

There was an improvement in ICF scores following rehabilitation. There were also improved FIM+FAM scores on discharge as compared to scores on admission.

The next chapter focuses on qualitative findings of the study. 


\section{CHAPTER 5: QUALITATIVE RESULTS}

\subsection{Introduction}

This chapter explores the knowledge of participants regarding the Occupation Health and Safety Act (OSHA), the Compensation for Occupational Injuries and Diseases Act (COIDA) and health and safety issues in their area of work. It also explores compliance by participants to health and safety policies in the workplace, and their views regarding prevention of SCI in the workplace. The information in this section was collected using semi-structured interviews using the guideline presented in Appendix D. Five participants were conveniently sampled from both research sites. The major approach taken by the study was quantitative as the main aim was to elicit baseline information about work-related SCI in South Africa, needed when interrogating preventative interventions. However, the researcher was also interested in gaining some understanding of how occupational health and safety issues were handled by both the employer and employee. The results in this section of the study complement the quantitative component covered in the previous chapter. Due to few individuals with work related spinal cord injury admitted at the time of data collection, only five participants were included in the study. The reader is therefore cautioned in interpreting or using the results to generalise them to the population.

\subsection{Demographic Profile of Participants}

Table 5.1 summarises the demographic details of participants. All participants were males and all were employed in the private sector. Three of the five were employed in the construction and service industries, had five years (or less) of experience in the company where they were employed when injured and did not receive training on occupational health and safety.

Table 5.1 Demographic characteristics of participants

\begin{tabular}{|l|l|l|}
\hline Age & $15-25$ & 1 \\
\hline \multirow{2}{*}{ Gender } & $36-45$ & 3 \\
\hline \multirow{2}{*}{ Marital status } & $46-55$ & 1 \\
\hline \multirow{2}{*}{ Level of education } & Males & 5 \\
\hline \multirow{3}{*}{ Industry sectors employed } & Females & 0 \\
\hline & Married & 3 \\
\hline & Widowed & 1 \\
\hline \multirow{2}{*}{$\begin{array}{l}\text { Years of experience in the } \\
\text { company }\end{array}$} & Never married & 1 \\
\hline & Primary & 2 \\
\hline & Secondary & 3 \\
\hline & Cervice & 0 \\
\hline & $0-5$ & 1 \\
\hline
\end{tabular}




\subsection{Themes Emerging From Qualitative Data}

Table 5.2 summarises the themes that emerged during the interviews. This is followed by a discussion of these themes with narrative examples.

Table 5.2 Summary of the categories and themes

\section{THEMES}

Poor compliance to health and safety regulations in the workplace

Recommendations to improve health and safety in the workplace (Employee's perspective)

Management of an injured employee in the workplace

\section{CATEGORIES}

Safety measures and procedures not adhered to

Employees involved in risk taking behaviour

Poor use of personal protective equipment

Poor or inadequate training regarding occupational health and safety

Poor working conditions

Fear of victimisation

The role of the employer

The role of the employee

Poor knowledge in accessing compensation benefits

Remuneration of the injured employee during hospitalisation or in-patient rehabilitation

Employer's support during inpatient rehabilitation

Perceived outcomes of rehabilitation

\section{Impact of rehabilitation}

\subsubsection{Poor compliance to health and safety regulations in the workplace}

\subsubsection{Safety measure and procedures not adhered to}

The participants reported instances of non-compliance by both employees and employers to health and safety regulations provided in the OHSA. For instance vehicles used by employees to deliver goods were not in good condition or not roadworthy. (Numbers in brackets following quotes refer to each speaker.)

"This truck we were using had no brakes; we had to use a brick to make it stop." "They told us the truck was not braking properly but we persisted and used it." (2). (The truck driver and the assistant were told by the supervisor and by fellow employees that the truck was not braking.) 
"The trucks we use sometimes you will find that they don't have brakes, we use the gear to brake it. Sometimes employees do not follow the right procedures when performing the tasks of their duties. The driver was driving with a speed of 95 and the limit was 90. The time the truck was overturning, I was not wearing a safety belt. At times the tyres are worn out..." (3)

\subsubsection{Employees involved in risk-taking behaviours}

The participants also reported incidents of they engaged in activities that would expose them to injuries or diseases in the workplace. This was especially evident in quotes form workers form the agricultural and construction industries.

Agricultural:

"The truck has no brakes; we had to use bricks to stop it .... The slope was very steep...I was standing behind the truck, it rolled back and hit me and I fell into a ditch." (2)

"Where I work it's hard. I used to get into the river, the water is pumping hard and there are other dirty things in the water. I just put on an overall and get into the water barefoot to save the calves. The boss usually tells me I going to get sick but I never did. I just developed a rash.” (2)

Construction:

"When we deliver goods, when we offload the goods, the employees sometimes throw the goods at you in any manner. I don't think that is safe because at times they will throw in a manner in which you are not expecting." (3).

"I was not wearing a safety belt when the truck overturned." (3)

\subsubsection{Use of personal protective equipment (PPE)}

The majority of the participants did not mention the use of PPE. Only two participants were aware of PPE and when to use it. One participant indicated that the employees had to buy the PPE.

When asked to name PPE he was familiar with (1) responded "Have to have the safety helmet, safety boots, overall... not to wear torn PPE. Vest, overall, helmet, gloves, shoes. The company pays for PPE."

"I used to just wear an overall; we pay for the overalls ourselves. Nothing is for free. Ayikho eyamahala." (2) 


\subsubsection{Poor or inadequate training on occupational health and safety}

The majority of participants indicated they did not received training on occupational health and safety, or that it was done inadequately:

"We went to training about health and safety but did not get the material to refer to. We do not have someone who looks at safety at work." (1)

"I heard about it (OHSA) but I never pay attention...There are no health and safety rules...There is no one who looks after health and safety where I work." (2)

"The rules they tell us upon signing the contact are related to how they are going to pay me when I am injured, some of the rules don't make sense, it's like they are forged." (3)

"There are no policies at work." (5)

\subsubsection{Poor working conditions}

Some of the participants indicated poor working conditions that put them at risk of sustaining injuries.

"Where I work things are tough. Eish man! The water comes very strong and it comes with lots of debris of rotten stuff... but I have to go in and save the calves." (2)

"The traffic is very busy; it's easy to get an accident here." (5)

"The work we do is not safe, because we work in dark places where its not easy to see your little finger. And they give you a torch. That on its own makes you vulnerable as its easy to be identified by the robbers." (4)

"Sometimes when offloading the goods, we throw them to each other into the storage from the truck."(3)

\subsubsection{Fear of victimisation}

Some participants expressed feelings of fear of victimisation if they raised issues of health and safety in the workplace:

"Sometimes you feel like telling the employer this and that is not right but there is one problem: we are afraid that we might get fired - things like these 'are not our business' but we end up injured." (2) 


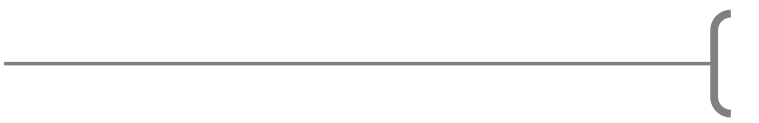

"Maybe we talk about a union that will protect us but there are those that will go behind our backs and tell the boss and the next thing you are under a watchful eye, one mistake you do, you are out." (3)

\subsubsection{Management of an injured employee in the workplace}

\subsubsection{Poor knowledge of accessing compensation benefits}

The majority of participants had a poor knowledge of compensation benefits and how to access them. Only one participant had a vague idea about the benefits. Participants were asked to share some information about the COIDA and the majority said: "I don't know" (4) (5) (2).

"It is the money you get following an injury at work. It is divided into two: you get money after the injury and they also pay for the medical bills." (3)

"I did not get my benefits, as the employer is saying that I was not employed by them but by the agency. "The forms [employers' report] has not been signed. They are now turning around and saying I was not employed by them." (1)

\subsubsection{Remuneration of the injured employee during hospitalisation and in-patient rehabilitation}

The majority of participants expressed concern regarding their salary during this period; but only one participant did not receive his salary during the period of in-patient rehabilitation. Although the majority of participants did receive their salaries in some cases it was halved.

"They are paying me at work but they are reducing it." (2)

"I get my salary but it is halved." (4)

"Yes I'm getting my salary." (5)

"The money they give me to assist my parents to visit me, they deduct it from my salary." (3)

"I am not receiving my salary." (1)

\subsubsection{Employers' support during in-patient rehabilitation}

Three participants reported there was no support from the employer during rehabilitation. However, two participants did receive support from their employer: 


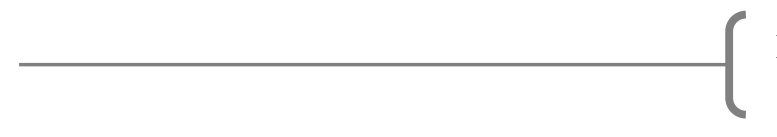

“There is no support from work.” (5)

They brought me here; they are paying for me here. Furthermore, they don't care about me anymore. My parents cannot come to visit me because of financial reasons, when I approached the employer they said that they will deduct the money from my salary to assist my parents to visit me." (3)

"I don't get any support from work. They don't even visit me." (1)

"They do support me. On the day of admission, the supervisor came to visit me and later my boss came to visit as well. They brought me clothes to change. They told me not to worry they are with me." (4)

"The boss gives my family a bag of maize meal. The boss came to visit and brought me toiletries." (2)

\subsubsection{Impact of rehabilitation}

\subsubsection{Perceived outcomes of rehabilitation}

Only one participant expressed positive feelings regarding in-patient rehabilitation.

"The level of quality of rehabilitation here is high and they are of great assistance. On admission, I could not do anything. The hand was moving a little bit but now it can reach my mouth. I can feed myself and be able to do some other things." (1)

"We are exercised there. Izinto azifani. Things are not the same. Shame. Bayazama bona ukuthi izinto zibengcono. They are trying to make things better. (3)

\subsubsection{Recommendations to improve health and safety in the workplace}

The participants made some recommendations on how to improve health and safety in the workplace. This section is divided on the role of the employee and the employer.

\subsubsection{The role of the employee}

"You have to wear a safety belt, drive with appropriate speed, and obey the road signs., When loading goods make sure it is tightly fastened, you must wear safety helmet, safety boots and an overall. When uploading must check your load if it is properly fastened so that it does not fall back on you. When at work one must wear work clothes at all time. Must follow health and safety regulations." (1) 
"Nothing can be done, you must protect yourself. You must be wise and protect yourself. You must check if you can be able to come out if you go in." (2)

"We must work as a team; we don't have much team spirit." (3)

"I cannot tell them what to do to prevent injuries." (5)

\subsubsection{The role of the employer}

"We must be taught about safety." (1)

"Something like, safety; most importantly on the trucks. Everything must be checked: wheels. They must change them before an accident happens."(3)

"I think there should be measures in place to prevent this from happening again. The employer must inspect the work environment for safety at all times. Can I work alone in a shift as a security guard or not? I must not use my eyes and hands to guard the area; I need a gun to protect myself. The employer must take care of us, when there is a problem they should not desert us."(4)

"I cannot tell them what to do to prevent injuries." (5)

\subsection{Chapter Summary}

Participants in this phase of the study were all male. The findings in this phase of the study indicate that both employees and employer carried a degree of responsibility regarding non-compliance to safety standards in the workplace. Safety measures and procedures were not adhered to, employees engaged in risk-taking behaviours and there was poor use of personal protective equipment. Participants also indicated poor working conditions. This was possibly due to poor knowledge of the occupational health and safety legislation. Participants expressed a need to be trained about occupational health and safety in the workplace and the maintenance of a safe working environment by the employers, and revealed fear of victimisation if they spoke out on these issues. Due to the small sample in this phase, the findings cannot be generalised to the population.

The next chapter focuses on the discussion of the findings. 


\section{CHAPTER 6: DISCUSSION}

\subsection{Introduction}

In this chapter the results of the study are discussed in relation to the aim and objectives of the study. Quantitative and qualitative results will be integrated during the discussion. Study findings will also be compared to literature findings. As previous studies on work-related SCI came from developed countries the findings with regard to general epidemiological information on SCI in South Africa, Africa and other developing countries will be used in the discussion. The researcher realise that qualitative findings were limited due to the challenges with regards to permission to collect data in the various companies. In addition the nature of Qualitative research is such that findings relate to a specific context at a specific time and cannot be summarily extrapolated to other settings or populations (Domholt, 2005). Thus he recommends cautious use of this information.

\subsection{Demographic Profile Of Participants}

\subsubsection{Gender}

The male-to-female ratio was at 6.69:1 (Table 2.5), which is lower than in international studies on work-related SCI (Ekong and Tator, 1985; Rosenberg et al., 1993; O'Connor, 2001, Webster et al., 2004). However, these studies showed a steady increase over the years in the number of females who sustained SCI in the workplace.

This increase as well as the lower ratio in the current study could be due to the fact that women are entering predominantly male-dominated occupations such as transportation and material movers; handlers, equipment cleaners, labourers; farming, forestry and fishing as well as protective services in ever increasing numbers in the USA (Wooton, 1997) and in Africa (Tzannatos, 1998). These were the occupations affected by SCIs in developed countries (Ekong and Tator, 1985; Rosenberg et al., 1993; O'Connor, 2001; Webster et al., 2004) and in the current study. In South Africa, this change may have been further supported by the change in the National Constitution and labour laws that promote gender equity, amongst others in employment practices (Employment Equity Act, 1998).

\subsubsection{Age}

Almost $63 \%$ of participants in the current study were aged between $26-45$ years, with the highest peak $(38.5 \%)$ in the age range 26-35. As indicated in Table 2.5, these age ranges are similar to those found in studies on work-related SCI in developed countries (Ekong and Tator, 1985, Rosenberg et al., 1993, Webster et al., 2004, O'Connor, 2007). The most affected age range also coincides with findings for general SCI populations in South Africa and Africa (Odendaal, 1991a; Odendaal, 1991b; 
Hart and Williams, 1994; Velmahos et al., 1995; Levy, Makarowo, Madzivire, Bhebhe, Verbeek and Parry, 1998; Igun, Obekpa, Ugwu and Nwadiaro, 1999; Frielingsdorf and Dunn, 2007; Udosen, Ikpeme and Ngim, 2007; Obalum et al., 2009; Emejulu et al., 2009).

The most affected age group in this study are individuals that are most likely to actively participate in the open labour market. Sustaining a spinal cord injury will limit their opportunities of gainful employment and that will increase a burden on the social security of the country as these individuals will have to access disability grants or compensation from the government.

\subsubsection{Marital Status}

Most of the participants in this study were either married (51. \%) or had never married (44\%). There is a paucity of literature on the marital status of individuals with SCI in South Africa and Africa and indeed in most SCI epidemiological studies. A study that did focus on marital status in SCI found a correlation between quality of life and marital status; it was demonstrated that individuals with SCIs who were single reported poorer quality of life than their married counterparts (Chan, 2000) . Furthermore, it has been demonstrated in the USA that a higher proportion of individuals with SCI are single or divorced after the occurrence of the SCI than in the general population (DeVivo, Hawkins, Richards and Go, 1995).

Sustaining a SCI may act as a stressor upon family and marital relationships as there has to be reallocation of resources and tasks within the family. Some spouses find it difficult to assume the caring role (especially males) and have huge difficulty adjusting to the new roles (Alexander, Hwang, and Sipski, 2002). SCI often increases the burden of care and perceived stress on the spouse, especially if the spouse is not well equipped with coping skills and strategies (Karan-Zebari, de Leon, Kalpakjian, 2011). The most cited positive predictors of marriage duration following SCI are age, higher education, being Caucasian, having stable health and greater social integration (Karran-Zebari et al., 2011). The implication for rehabilitation is that attention must be paid to the latter two factors to make sure that they are included in the overall aims and goals of rehabilitation of an individual with SCI, to try ensuring families and marriages do not dissolve and that interpersonal relationships are not strained following SCI. The positive aspect of being married before sustaining SCI is the support received from the spouse, although it comes with enormous stress to them. Their support helps in removing the barriers that prevent progression in the rehabilitation efforts of individuals with SCI (Karran-Zebari et al., 2001). 


\subsubsection{Population Group}

The majority of the participants in this study were Black African (79\%). Similarly, Statistics South Africa (2011) estimate the total percentage of Black South Africans at $79.5 \%$. Thus the figures might simply be a reflection of the country's population numbers.

Another factor might be that, according to UNESCO (2008), Blacks are less educated than Caucasians. This results in their participating in less desirable and more labour-intensive occupations, which expose them to greater risks of sustaining occupation-related SCIs. Blacks were in the majority in most of the occupations that caused SCI in this study. Findings indicated that $100 \%$ of security guards, $87.5 \%$ of labourers, $92.9 \%$ of miners and $82.4 \%$ of drivers were Black. Although this study did not gather information on the education level of the participants, since that data was not readily available in folders, it is difficult for an individual without formal education to return to work following a SCI, especially in the occupational categories mentioned above (Ottomanelli and Lind, 2009). Re-employment after injury and the factors impacting on that will be discussed in detail under Section 6.11 .

\subsection{Occupations And Industries Affected}

This study found that the service industry, engineering, mining and construction, in that order, were the four industries most affected. These results are similar to a study published in 2004 by Webster et al., in the United States. The service industry included the bulk of the security cluster (security and police officers), the third most affected occupational group (20.8\%).

This could be due to the high level of violence that is associated with this cluster. According to the Gauteng Safety Strategy 2006-2014, violent crime undermines the quality of life for many people in Gauteng. Violent crimes in Gauteng include murder, attempted murder, rape, serious assault and all forms of robbery. This high level of crime and violence necessitates preventative interventions by business owners (e.g. security guards) and the general public (policemen). The Strategy confirms that firearms are often used in crime and robberies. This exposes security guards and policemen to the risk of being shot while attempting to apprehend a perpetrator, as was the case for 26 (19.3\%) participants in the current study.

In this study, labourers were the most affected employment group in engineering (60\%), the construction industry (69.2\%) and agriculture (77.8\%). Labourers were also the occupational group most affected overall (25.9\%). The majority of the labourers were Black. Labourers and those in related occupations were also the most affected by SCIs in Australia (O'Connor, 2001). Low-skilled and labour-intensive occupations are at risk of incurring SCIs (Ekong and Tator, 1985; Rosenberg et 
al., 1993 and O'Connor, 2001). Labourers in these industries are exposed to falls, being hit or being struck by an object or piece of machinery or by a falling object. Qualitative findings of the current study indicate that employees in the agriculture and construction industries were not compliant with health and safety regulations in the workplace, which increased their risk of injury.

In the mining industry miners were the occupational group to be most affected, at $73.7 \%$. This was to be expected since miners often work underground in conditions were rock falls can occur. The most affected workers in the manufacturing and transport industries were drivers. This could be due to noncompliance with road safety regulations, including driving within the speed limit, wearing safety belts and roadworthiness of the vehicles, all of which were problems identified during qualitative interviews.

The occupation with the second highest number of injuries was the professional group (23.7\%). The researcher find this surprising since one usually associates professional work with more sedentary, office-bound work with fewer of the common risks for SCIs. The industry in which professionals were most injured was business (91.7\%). However, further information is needed to ascertain the reasons for the high number of professionals being injured in this and other industries.

Although further exploration is necessary qualitative data indicated the possibility of non-compliance of employers and employees with health and safety regulations. Specifics found in this study included non-compliance of employers (by not providing safety equipment or ensuring roadworthy vehicles) and of employees (not wearing appropriate personal protective equipment (PPE) or adhering to health and safety regulations). A study conducted in Taiwan indicated that employers' (small and medium) lacked general awareness relating to issues of health and safety in the workplace (Hu et al., 1998). They found that lack of knowledge and a negative perception of the importance of health and safety in the workplace leads to non-compliance with health and safety regulations. This might be true for the current study population as well, but was not assessed.

The OHSA stipulates the roles and responsibilities of both the employers and the employees with regard to compliance with health and safety regulations in the workplace, including that of employees with wearing of PPE. Literature also indicates a lack of compliance with wearing PPE (McPherson and MacDougall, 2006). According to McPherson and MacDougall (2006) this non-compliance can be attributed to a feeling of invulnerability among workers, lack of awareness of workplace hazards, lack of training on proper use of PPE and ill-fitting and uncomfortable garments. Akbar-Khanzadeh et al., (1995) alluded to the fact that for the workplace to be safer, employers have to enhance the comfort, protective qualities and form or style of PPE, while providing employees with adequate education and personal protective programmes. 


\subsection{Causes Of Work-Related Spinal Cord Injuries}

The major cause of work-related SCI in this study was motor vehicle accidents (MVAs). The second most common cause was violence. Falls, being hit by a falling object or being struck by an object or piece of machinery were the least common causes of work-related injuries. This is in contrast to studies done in developed countries (Unites States of America, Canada and Australia) where falls, followed by MVAs, were the major cause of injuries; violence as a cause was very low (Ekong and Tator, 1985; Rosenberg et al., 1993, Webster et al., 2004, O'Connor, 2007).

Studies on non-occupation related causes of SCI in developed countries found traffic accidents to be the most common cause of injury, ranging between $35-53.8 \%$, while falls were the second highest cause of SCIs at between 22.6-37\% (Nobunaga et al., 1999; Sekhon and Fehlings, 2001; Ackery et al., 2004, Pickett et al., 2006).

In South Africa, MVAs are also the highest cause of SCI with a range of 25-63\%, followed by violence (8-56\%) and falls (2.4-28\%) (Odendaal, 1991a; Odendaal, 1991b; Hart and Williams, 1994; Velmahos et al., 1995 and Frielingsdorf and Dunn, 2007).

Studies on non-work-related SCIs done in Nigeria yielded similar results: road traffic accidents as the major cause ranged between 57-89\%; falls were the second highest ranging between 4-31\%, while violence (gunshot and stabbing) ranged between 4-7.3\% (Igun et al., 1999; Solagberu, 2002; Olasode et al., 2006; Udosen et al., 2007; Emejulu et al., 2009 and Obalum et al., 2009). Similar results were also found in Zimbabwe (Couto et al,1994; Levy et al., 1998).

Factors that may influence the high number of motor vehicle-related SCIs in Africa could be the underdeveloped infrastructure and general non-compliance of the population with road safety regulations (African Union-Programme for infrastructure Development in Africa, 2012). In the work environment, non-compliance of employees and employers with these regulations could be a leading factor of SCI. Responses gathered from the participants during the qualitative phase of this study also pointed towards challenges in this area. Time pressure exerted by clients and employers on drivers to make deliveries on time might cause speeding. One of the other contributing factors to motor vehicle accidents can be that police and security officers often drive at high speed when responding to a call out or pursuing a suspect, thereby increasing the chances of an accident.

Factors that may have led to the high number of individuals who sustained SCI due to violence include general high levels of crime in Africa and South Africa (McDonald, 2008, Van Niekerk, Suffla, Seedat, 2008.) and the inherent nature of certain jobs for instance being a security officer or 
police officer exposes the law enforcer to risk of violence (Mohammed, Edino, Ochicha and Umar, 2005).

However, accidents at work should not happen, irrespective of the cause, as safety procedures have been developed (see the OHSA) and should be strictly adhered to in any occupation or work environment to prevent occupation-related injuries. One of the strategies that employees identified to prevent injuries in the workplace is adequate training regarding health and safety in the workplace. Another is that employees should actually adhere to regulations such as wearing safety belts, speed limits and other traffic regulations, as well as wearing protective clothing and shoes. Some personal and interpersonal aspects were also pointed out, such as protecting oneself, not taking risks and working as a team and not against one another.

Employers have a responsibility to ensure that their vehicles are roadworthy and their employees are provided with appropriate safety equipment and are supported and protected; for instance, security guards need back up and may need a weapon for self-protection in certain situations. Employers have a responsibility to ensure that safety regulations are adhered to and they need to do actual inspections to see whether these measures are being followed.

It would have been very interesting to understand the perspective of the employer. This proved impossible as the employers approached refused to participate in this study. This may have been due to fear of being exposed if the researcher found instances of non-compliance with OHSA or of negative publicity. The researcher did state clearly in the information letter that this would not be the case and that all participants and companies would remain anonymous.

\subsection{Occurrence Of Spinal Cord Injuries In The Study Setting}

This study was an institution-based study as the researcher was refused permission to access files at the Compensation Fund, a division of the Department of Labour. This department has a database of most injuries that occur at work. In 2000, the first year of the study period, a low number of SCIs patients were admitted to the study hospitals which steadily increased to a high in 2009 (Figure 4.3). This may be due to a number of factors including improved marketing strategies of the facilities; inadequate resources to deal with a large number of patients initially; and poor financing from healthcare funders (the medical aid societies and the Compensation Commissioner) especially since private rehabilitation in South Africa is a relatively young concept that only really started in the late nineties. There was a sharp increase from 2007 to 2009. This could be due to addressing the factors mentioned above and branding of the rehabilitation facilities. However, since this is an institutionbased study as opposed to a population-based one, no inferences can be drawn with regard to trends in numbers of occupational-related SCI over the years of study. 
The majority of the injuries in this study occurred during the day, $45 \%$ in the afternoon and $33 \%$ in the morning. Most companies in South Africa operate between 8:00 am and 16:30 pm, which fits in with the hours of injury.

As already stated, MVAs are the highest cause of work-related SCIs in this study. In Gauteng, it is obvious from observation that there is highest traffic density in the early morning and late afternoon as employees are rushing to work, drivers are rushing to deliver goods and, in the afternoon, everyone is hurrying to go home.

It appears the risk for injury is lowest mid-week with an increase in risk from Thursday through to Monday and a dip on Saturdays; most injuries occurred on Fridays. In South Africa, for most industries this is the last working day of the week. It is possible that rushing to finish deliveries might increase speeding and general tiredness might decrease concentration and increase the risk for accidents, both on the roads and in other work areas. On Friday afternoons buildings also empty for the weekend, except for security guards who must protect the property and its contents. Thus the risk for robbery and injury to security personnel can be seen as higher on Friday to Sunday, another day that showed a high risk for SCI. However, the fact that Saturday did not show such a high risk is difficult to explain, as it should show a similar risk profile to a Sunday when many businesses would only be performing essential duties or would be closed for the day.

In this study, the most common months in which SCI occurred were February and April and the least common was December. December is generally a down-time for many industries as schools close from the first week in December until mid-January, and most employees take leave between Christmas and New Year. Mid-December to mid-January is the holiday period of the national building and construction industries. Between January and February there is usually a steady increase in productivity. Furthermore, in December, there are widespread campaigns on road safety in South Africa focussing on holiday traffic. These campaigns may have increased awareness and contributed to a decrease in work-related MVAs at this time.

\subsection{Level and Classification of Spinal Cord Injury}

The most affected regions of the spine in this study were cervical $(41.5 \%)$ followed by the thoracic spine (34.8\%). The majority of the participants (51.1\%) had complete lesions (ASIA A). The majority (53\%) of the participants with incomplete lesions were classified as ASIA D i.e. motor function is preserved below the neurological level and at least half of the key muscles have a muscle grading of $3 / 5$ on the Oxford Muscle Grading Scale. The majority (58.4\%) of the participants were classified as being paraplegic. 
Thus the results of this study are consistent, in some of the aspects mentioned above, with studies done in developed countries on work-related SCI. (The figures for non-work-related SCIs in South Africa also showed that paraplegia was most common (64-74\%). (Hart et al., 1995 and Velmahos et al., 1995.) A study done by O'Connor, 2001 in Australia revealed 67\% of the participants as paraplegic and literature from the USA, Rosenberg et al (1993), found 56\% to be paraplegic, but a study from Canada yielded contrasting results (57\% tetraplegia) (Ekong and Tator, 1985). The differences in the findings might be due to the different mechanisms of injury in the various study settings (Generally more injuries in the study by Ekong and Tator (1985) were caused by falls and struck by objects than in the other studies).

The level and completeness of the SCI are important since it impacts on a person's functional ability and the length and cost of rehabilitation. These aspects will be discussed in sections that follow.

\subsection{Rehabilitation Provided To The Study Population}

The results showed that rehabilitation was provided through individual and group sessions. Families were educated and trained in $87 \%$ of cases. With the devastating effects of a SCI and the impact it has on both the individual and the family (as discussed in Chapter 2) one would have liked to see training and education of all families. However, it is possible that some of the participants whose injury was classified as ASIA-D did not have any long-term activity limitations that impacted on their functionality, which may have lead the rehabilitation team to decide that family training and education was not necessary. Another reason would be that some families lived too far away and could not access the rehabilitation facilities due to financial constraints.

All participants received medical rehabilitation. This included a variety of techniques to improve activity limitations and impairments. Chest physiotherapy was performed in 57\% of cases. This is consistent with the finding that $56 \%$ of the participants suffered cervical injuries. With a cervical injury respiratory muscles are involved, compromising breathing and coughing, often necessitating chest physiotherapy. Other findings that are consistent with the literature are those on balance, strengthening and pressure care.

Eighty-four percent of participants received gait re-education. This is a high number if one considers the finding that $49 \%$ of participants could not walk at all according to ICF findings. When one further considers that all participants were taught wheelchair activities, 99\% did therapeutic standing and $98 \%$ were taught transfers while only $69 \%$ received wheelchairs on discharge it does seem as if rehabilitation and treatment goals was not as focused on individual patient needs as they could have been. 
Diet therapy was also provided for all participants. This is a positive finding since diet is an important aspect of management in a condition that affects the nutritional system in various ways. SCI affects bowel function (Krassioukov, Eng, Claxton, Sakakibara and Shun, 2010). In addition, SCI individuals have an increased risk for obesity due to being inactive (Chen, Henson, Jackson and Richards, 2006). Dietary guidelines both in terms of type and amount of food being eaten are thus essential.

Another positive finding is the provision of counselling to all participants. As discussed in the literature review SCI can be seen as devastating and life transforming (Paddison and Middleton, 2005) and as such counselling is essential to support the person through the process and prevent or deal with psychological challenges such as post-traumatic stress, anxiety and depression which occurs in up to $37 \%$ of SCI individuals (Migliorini, Tonge and Talepos, 2008).

One finding on the rehabilitation services offered which was less positive was that none of the participants received vocational rehabilitation. The need for and importance of this will be explored under the section dealing with re-employment.

Assistive devices are important tools used in rehabilitation to improve the functionality of individuals with various disabilities. In this study, wheelchairs and cushions were the most issued assistive devices. This is to be expected in a study population where $51 \%$ suffered complete SCI and $23 \%$ suffered an incomplete injury with non-functional motor sparing; this is consistent with the finding according to the ICF scales that $57 \%$ of participants could not walk at all or found walking very difficult. Wheelchairs will improve mobility of these participants and will in turn improve their participation in life and social roles (World Health Organization, 2008). Another positive finding is that all participants who received wheelchairs received wheelchair cushions. The cushion is essential in optimising the seating posture in the wheelchair and preventing secondary complications such as pressure ulcers (Western Cape. Department of Health, 2009).

It is disturbing to note that individuals in the study were not issued with many assistive devices to improve activities of daily living. A large proportion (41.5\%) had cervical injuries, with anticipated loss or impaired function of the upper limbs. Functioning can be enhanced by devices such as splints and extended handles. Pressure ulcers are a common secondary complication following SCI (Kroll, Neri and Ho, 2007). The risk for these can be decreased through various practices and provision of a special mattress. Four percent of participants received such a mattress yet $17.8 \%$ had a complete cervical lesion. 


\subsection{Duration Of In-Patient Rehabilitation}

Length of stay (LOS) is often viewed as an indicator of the efficiency of healthcare delivery. It is reasoned that shorter stays with similar functional outcomes are the result of effective management and organisation of resources (Eastwood, Hagglund, Ragnarsson, Gordon and Marion, 1999). The mean LOS was 66 days, with the LOS ranging from 9 to 350 days. Research findings vary on the LOS in rehabilitation of patients with SCIs. The mean LOS in this study was shorter than most of studies published elsewhere. Two studies in the Netherlands report on a mean stay of 228 days (Osterthun, Post and van Asbaek, 2009) or 275 days (Post, Angenot, van Asbeck and van der Woude, 2005). A study done in Australia reported a mean stay of 83 days (Tooth, McKenna and Geraghty, 2003), and a study done in Italy reported 122 days (Pagliacci, Celani, Spizzichino, Zampolini, Aito, Citterio, Finali, Loria, Ricci, Taricco, and Franceschini, (2003). These differences in the average LOS might be attributed to the healthcare financing models of a particular country. Medical funders in South Africa limit the number of days a patient is allowed to stay for inpatient rehabilitation, though they periodically assess each case individually.

Participants in the current study with quadriplegia stayed longer (71.68 days) than those with paraplegia (60.05 days). Participants with cervical (71.68 days) and thoracic (67.98 days) lesions stayed longer than participants with lumbar (52.13 days) lesions. The difference in the mean LOS between the groups could be attributed to the fact that patients with tetraplegia have more functional limitations than patients with paraplegia, as patients with cervical lesions (tetraplegia) have less use of their upper limbs and trunk than patients with paraplegia. These findings were consistent with findings from a study by Osterthun et al., 2009, which found that patients with cervical injuries stayed longer (228 days) than those with paraplegia (160 days). In the current study, participants classified as ASIA C and B (Table 4.10) stayed the longest, although in other studies, patients with complete injuries stayed longer than patients with incomplete lesions; this was true of patients with both tetraplegia and paraplegia (Tooth et al., 2003, Pagliacci et al., 2003; Post et al., 2005 and Osterthun et al., 2009). This difference could have been because increased time was spent trying to improve the strength of muscles and walking ability before the patient was discharged.

The findings revealed that there was no statistical difference between the mean LOS of males and females, but females showed a much wider distribution of the LOS scores than males. These findings are similar to studies done by Tooth et al., 2003, Pagliacci et al., 2003; Post et al., 2005 and Osterthun et al., 2009. The difference in the distribution of the LOS mean scores could be attributed to the fact that females often suffer from more complications following SCI than males (Bennett, Young and Darrington, 1995). However, this phenomenon was not explored in this study. 
In this study, there was no statistical difference between LOS and the causes of SCI although there was a relatively low mean score on those with miscellaneous causes (falls, struck by an object etc.). This finding could be attributed to the fact that miscellaneous causes of SCIs in the workplace usually result in paraplegia (O'Connor, 2007).

The relationship between LOS and functional FIM+FAM scores in this study was marginal ( $\mathrm{r}=-0.32)$, indicating that the longer the LOS, the higher the FIM+FAM scores. Similar results were found in other studies (Tooth et al., 2003, Pagliacci et al., 2003; Post et al., 2005 and Osterthun et al., 2009). This relationship could be attributed to the fact that more time is spent with an individual, which often results in functional gains. However, this information should be used with caution when determining the LOS of patients on admission, as there are many factors to consider and more research is needed to establish a clear relationship between these two variables.

\subsection{Costs Of Rehabilitation}

The costs of rehabilitation in this study cannot be compared with those of other countries, due to the difference in the variables used to assess the costs (direct or indirect) and the difference in the currencies used.

Few studies included LOS as a major variable to determine the costs of rehabilitation of SCIs (Cifu et al., 1999a; Cifu et al., 1999b; Fiedler et al., 1999 and Dryden et al., 2004). The results in this study indicated that it is more expensive to rehabilitate patients with tetraplegia than patients with paraplegia. These findings show that it takes longer to rehabilitate an individual with tetraplegia than an individual with paraplegia and also that patients rated ASIA B had higher costs of rehabilitation than ASIA A. The only way to decrease these costs is by primary prevention of the SCIs in the workplace.

\subsection{Outcomes Of Rehabilitation}

Around $30 \%$ of participants experienced difficulties with the various self-care activities. It is to be anticipated that individuals with quadriplegia would experience some difficulty with self-care due to impaired upper limb and hand function and balance (20\% of participants experienced severe challenges with fine hand use), as most of the activities in this category require good functioning of the upper limbs and adequate sitting balance.

Lifting and moving or carrying objects was one activity that the majority of the participants could not perform. This is understandable when one uses a wheelchair or crutches for mobility. However, there is a real possibility that this score could have been improved through issuing of more lap trays. Only 
$4 \%$ of participants received these. A lap tray enables a person to carry at least some objects on his/her lap while propelling a wheelchair with the hands.

Doing transfers was one activity that the majority of participants could perform with no difficulty and is part of the medical rehabilitation programme, as shown in Table 4.21. One can deduce that this aspect of rehabilitation was effective.

Walking and standing up from sitting was impossible for 30-50\% of participants, as can be expected from a cohort of participants of whom 51\% suffered from complete SCI. Getting around with wheelchairs or devices was less challenging. Participants were able to move out of the house and around the house with minimum difficulty. This is a positive outcome as it plays an important role in determining the level of participation in life situations amongst people with SCIs.

In the community integration category, the majority of the participants could participate in various activities in the community with no difficulties. Fifty percent of participants could use transport and $45 \%$ could drive. Being able to drive is especially important in South Africa where public transport is notoriously inaccessible (Walters, 2008).

\subsection{Employment Status On Discharge}

A very positive finding was that $54.4 \%$ of participants went back into employment after discharge from rehabilitation. This is especially pleasing since the findings show that the cohort is a relatively young group that have the greater proportion of their working life ahead of them. The finding is seen as positive as it is comparative with international literature that indicates a wide range in reemployment rates varying from 27 to 67\% (Lidal, Huynh, and Biering-Sørensen, 2007; Ottomanelli and Lind, 2009; Young and Murphy, 2009). In developing countries usually sustaining SCI is associated with loss of employment, as there are few opportunities for vocational rehabilitation (International Labour Organization, 1998). This prevents the person with SCI from participating in the open labour market, which has a negative impact on the national economy as this cohort will join the unemployed and increase the burden of the state to provide social grants. In addition the unemployment figure amongst disabled persons in South Africa is high at 99\% (Dube, 2005).

One reason why a relatively high return to work figure was achieved might be the fact that all participants were injured on duty. Thus companies might have been faced with a choice between reemployment or the paying of disability benefits and chose the former. Although in companies where there are no structured disability benefits, it would be easier and cheaper for the employer to make sure that the employee applies for compensation benefits from the Department of Labour and the employee retire on medical grounds. The employer is well within their rights to dismiss employees 
who are incapacitated due to ill-health or injury on grounds of poor performance but the employer has to follow the guidelines as stipulated in the schedule 8 of the labour relations act short of dismissal. In addition persons with a job find it easier to return to that job than to find another job after disablement (Lidal et al., 2007).

Study findings were further analysed to try and identify factors that could have aided the relatively high return to work figure. The only factor found to have a statistically significant positive impact on re-employment was the level of the lesion with persons with lumber lesions more likely to be reemployed than those with thoracic or cervical lesions. This might be expected since a lumber lesion impacts the least on one's functional ability. However, a heartening $49 \%$ of participants with cervical and thoracic lesions were also re-employed. The ASIA classification had no impact on re-employment status.

The mining, service and engineering industries were willing to re-employ injured employees. All but one police officer went back to their previous employer, while only one security officer was employed again by his previous employer. It was surprising to find that $40 \%$ of labourers went back to employment since this is an occupation associated with physical labour and the need for physical strength. Miners and professionals were also well accepted back by previous employers.

Population group did not play a significant role in being re-employed or not. According to literature being Black is associated with a risk of not returning to work following an injury due to lower levels of education prior to the injury (Putzke, Hicken and Richards, 2002; Meade, Lewis, Jackson and Hess, 2004; Krause, Terza, Erten, Focht and Dismuke, 2012). However in this study $42.5 \%$ of Black participants went back to employment.

According to literature factors that lead to re-employment of individuals with SCIs include the following: high level of education, being Caucasian, younger at time of injury and having lived more years with SCI (Krause, Kewman, DeVivo, Maynard, Coker, Roach and Ducharme, 1999 and Lidal et al., 2007). These were not reflected in the current study. More research needs to be done to explore the mechanisms used by employers to accept employees who sustain work-related SCI back to work and also to ascertain the fulfilment of people with work-related SCI with the physical demands of their previous occupation.

Vocational rehabilitation was not offered in either of the study facilities. However, staff at these facilities did make efforts to meet the employer and to discuss alternative employment opportunities for the participants upon discharge. It could have a positive effect on the productivity levels of the participants if the rehabilitation facilities offered a vocational rehabilitation service to the participants. 


\subsection{Improvement In FIM+FAM Scores Between Admission And Discharge}

FIM+FAM scores showed a significant improvement from admission to discharge for all regions of the spine, except the lumbar region. This might be due to the fact that an injury to the lumbar region of the spine has the least impact on function. Studies done on functional outcomes found similar results, although the methodologies differed (Tooth et al., 2003, Pagliacci et al., 2003; Post et al., 2005 and Osterthun et al., 2009). The findings of this study, relating to the positive effects of rehabilitation in individuals with SCIs, can be used to motivate the financing of rehabilitation of SCIs by the medical funders (medical aids) who are often reluctant to pay for longer periods of rehabilitation as it is costly.

Often the outcomes of rehabilitation are assessed by excluding the individual's perceptions of his satisfaction about the rehabilitation services he receives. In this study, one respondent in the qualitative phase was satisfied with the rehabilitation received and was able to see the difference in his functional capabilities.

\subsection{Chapter Summary}

There is an increase in the number of females who sustain SCIs in the workplace, although males still dominate. Work-related SCIs worldwide affect the economically-viable age group. The majority of participants were Black Africans which correlates with the number of Black Africans in the South African population.

The service industry, including the security cluster, was the most affected industry with motor vehicle accidents and violence being the major causes of SCIs.

A large number of participants in this study sustained SCI of the cervical spine with grave functional limitations even though the majority of the lesions were classified as incomplete. The average LOS for rehabilitation was approximately 2 months and it was more expensive to rehabilitate those individuals who sustained tetraplegia, as it required a more prolonged stay. The outcomes of rehabilitation interventions were good as demonstrated by the marked improvement of the FIM+FAM scores on discharge. The majority of the participants were employed on discharge.

The next chapter concludes the study and provides recommendations. 


\section{CHAPTER 7: CONCLUSION AND RECOMMENDATIONS}

\subsection{Conclusion}

This study described work-related spinal cord injuries (SCIs) in Gauteng Province, South Africa, and the compliance of employers and employees with the rules and regulations of the Occupational Health and Safety Act 130 of 1993.

One hundred and thirty-five work-related SCIs in ten years (1998-2009, the period of the study) may sound low, but this data came from only two of the four private rehabilitation units in Gauteng, and does not account for those persons rehabilitated in other units (both private and state) or who were not referred to rehabilitation units. The incidence of work-related SCI in Gauteng over this period will therefore be higher than found in this study. Some of the injuries could have been prevented through the proactive implementation of sound health and safety procedures and adherence to national and company regulations. For instance the high prevalence of motor vehicle accidents as cause of injury leads to the conclusion that traffic and road safety regulations were not always adhered to by both employers and employees. While other road users might have been the guilty party in some instances the findings on failing brakes and non-use of safety belts points towards challenges within the study population. Therefore a nationwide approach to road safety might be adopted. Both employers and employees were found to be non-compliant with road safety and other health and safety practices in the workplace, which could have led to the occurrence of SCIs. Preventative strategies should be specific to different occupational settings, and targeted to specific age groups. Given the increase in the number of women suffering SCIs, these strategies should also be gender-specific in response to differences in males and females in terms of determinants of health.

Compliance to safety regulations and prevention of SCI is of utmost importance if the cost implications, both in money and functional outcomes, are taken into consideration. While relatively high return-to-work rates were found in comparison to general employment rates of persons with disabilities in South Africa, rehabilitation interventions should include vocational rehabilitation to increase further employment rates of people who sustain SCIs in the workplace. This is especially important since the study findings indicated that work-related SCIs are often suffered by relatively young persons (26-35 years old) who have many productive years in front of them. 


\subsection{Recommendations}

\subsubsection{Preventative Framework}

A framework (Figure 7.1) is proposed for the prevention of SCIs in the workplace. This framework demands an integrated and multi-disciplinary and multi-sectoral approach to promote the prevention of SCIs in the workplace.

\subsubsection{Development of a national SCI register}

The primary recommendation is for the development of a National Register for SCIs sustained in all nine provinces and in various occupational settings, to ensure all factors that contribute to SCIs in the workplace are recorded and known. The Department of Health (both at national and provincial level) should take responsibility for developing, managing and maintaining such a register. The Department of Labour has a national database of reported work-related SCIs which it should feed into the National Register of SCIs. The Road Accident Fund (under the authority of the Department of Transport) should also have a register that would feed into the National Register. This coordinated development would make sure all information relating to SCIs is readily available to all stake holders.

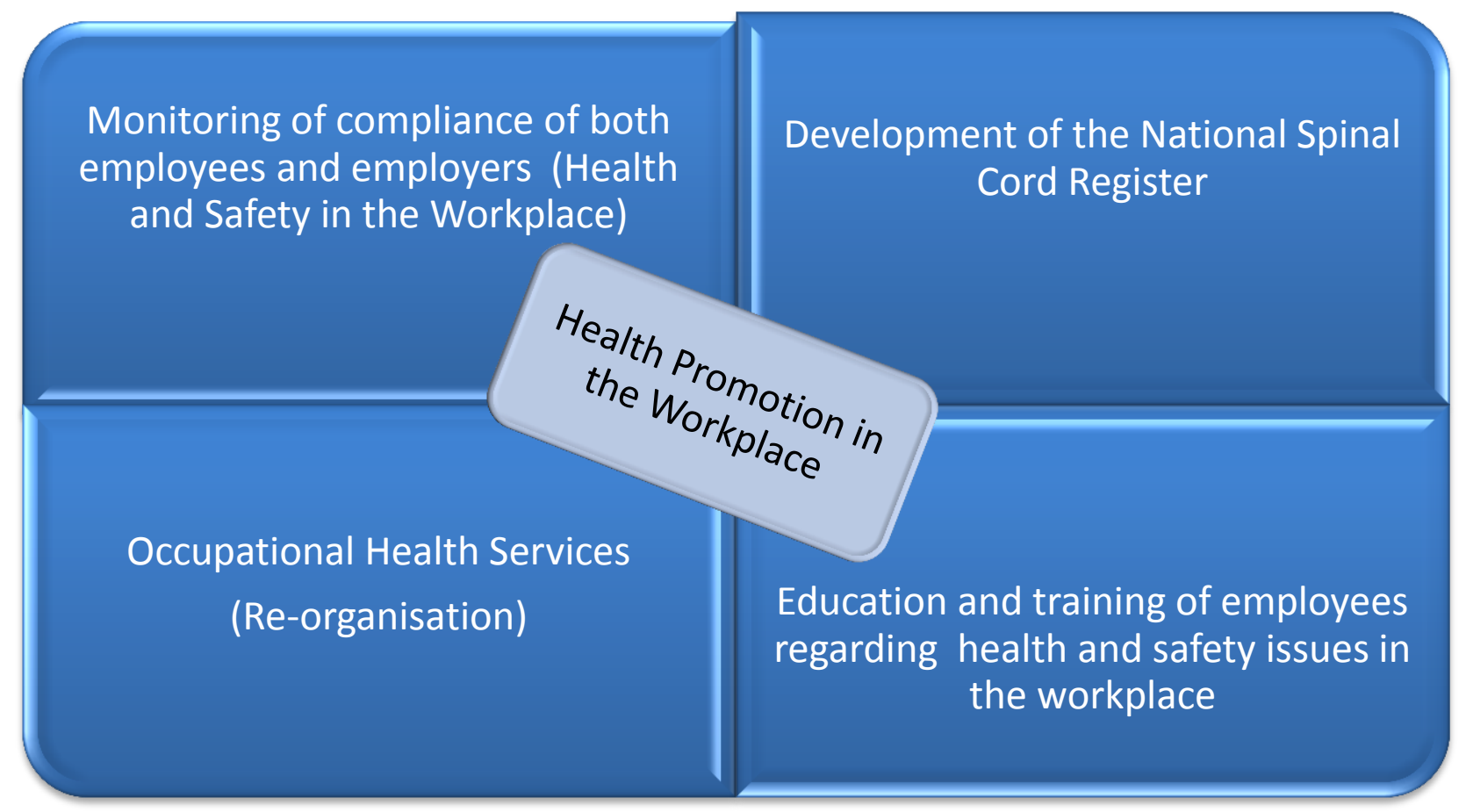

Figure 7.1: Proposed Preventative Framework 


\subsubsection{Monitoring compliance on health and safety in the workplace}

Measures should be put in place to monitor the compliance of employees and employers with health and safety regulations in the various industries. Regular and random compliance audits may be done by the designated inspectors from the Department of Labour. Section 28, 29 and 30 of the OHSA, 1993 clearly define the role of the inspector and the powers they have in executing this task. In instances of non-compliance, on-going support and training should be done with close collaboration of the various stake holders, including the occupational health sector, and, where necessary, legal action should be taken. Companies may also employ Occupational Health and Safety representative to make sure that employees and employer comply with health and safety regulations.

\subsubsection{Re-organisation of occupational health services in South Africa}

According to the White paper on transformation of the health system in South Africa of 1997, the provincial departments of Health have a major role to play in the provision of occupational health services to small and medium sized companies, and the public and informal sectors, through the district health system. One of the objectives of the proposed provision of occupation health services within the department of health is to develop occupational health education strategies with the aim of primary prevention of work related injuries or diseases, in this instance work related spinal cord injuries.

It is therefore imperative that the Department of Health should put in place measures to ensure that occupational health services (OHS) are accessible to all employees, irrespective of locality. Mobile Occupational Health Clinics should be used to serve employees who do not have access to in-house OHS. Adequate allocation of financial and human resources is required to serve this purpose. The Department of Health should engage with private companies offering OHS, through public-private partnerships, to alleviate the capacity challenges it would inevitably face. Primary prevention of workrelated SCIs should be a high-level goal of OHS in addition to rendering other occupational health services.

One strategy to meet this goal could be requiring rehabilitation professionals to be part of health promotion initiatives in the workplace. This would expand the scope of practice of all rehabilitation professionals, most especially physiotherapists and occupational therapists. South African universities training these therapists should embrace this as an opportunity to enhance the training and expertise of future physiotherapists and occupational therapists by developing and promoting courses in Occupational Health. 


\subsubsection{Educating employees on health and safety in the workplace}

The development of Health promotion programmes in the workplace should include the following:

- Training employees on the expectations of the law regarding health and safety in the workplace, including their responsibilities with regard to injury prevention and their rights in case of injury;

- Self-care strategies to prevent work-related injuries. Self-care strategies include education of individual employees in an attempt to change their behaviour towards compliance to health and safety regulations in the company in an attempt to prevent spinal cord injuries in the work-place. This may include education about road rules and regulations, road trip preparation, handling of equipment, regular use of PPE (e.g. bullet proof vests for the security cluster employees, harness for the construction employees) and self-defence classes for the security cluster employees.

- The health education strategy to be employed in this initiative will be the Trans-theoretical model of health behaviour change (Prochska and Velken, 1997).

\subsubsection{Recommendations To Employers (In relation to Section 8 (1 and 2) of the OHSA, 1993)}

- Employees should be adequately and periodically trained in matters of health and safety in the workplace. This should be done at various levels (executive, middle management and general employees) to accommodate all employees. Training could be arranged internally through the Human Resources department in partnership with the Department of Labour or it can be outsourced from various accredited training service providers.

- Employers should regularly inspect all machinery and equipment used by employees to perform their tasks, to ensure it is in safe and compliant working order. Vehicles used to transport employees or merchandise should be serviced regularly and be certified roadworthy. Employers should develop a checklist to ensure all aspects of safety are checked and recorded, ideally on a daily basis.

- Quality control and assurance audits should be done to ensure compliance. This could be achieved through the engagement with the Inspection Directorate of the Department of Labour and the National Centre for Occupational Health of South Africa.

- A working environment conducive to safety should be created. This environment should be free of hazards or practices that could expose employees to sustaining SCIs. For example: all vehicles should have a device that monitors use of safety belts, speed and the quality of driving. 
- All employees should have access to personal protective equipment (PPE) provided and maintained by the employer at the employer's expense.

- Information on prevention of work-related injuries should be made available and accessible to all employees. Posters can be put up on the causes of SCIs in a particular industry; for instance, in the construction and mining industries posters depicting the prevention of slips and falls and poor ergonomics should be made visible.

- Employees should be trained about their rights and responsibilities in the workplace. Special emphasis should be place on the compensation benefits payable should they get injured at work, the process of rehabilitation and opportunities for re-employment following a SCI. Public-private partnerships between the Department of Labour (skills development), the Skills Education Training Authorities (SETAs), and rehabilitation professionals (vocational rehabilitation) could achieve this aim.

\subsubsection{Recommendations To Employees}

- Employees should ensure they comply with the rules and regulations of health and safety in the workplace and understand the consequences of transgressing these.

- Employees should carry out daily inspections in their own area of work to ensure that all equipment and machinery (including vehicles) is in good working condition before starting work.

- They should practice safe ergonomic procedures at all times when performing their occupational duties.

- They should comply with PPE requirements at all times.

- They should also make sure, through their shop stewards and union representatives that they receive training regarding health and safety.

\subsubsection{Recommendations To Rehabilitation Facilities}

- Vocational rehabilitation should be included in the rehabilitation package to assist individuals suffering a work-related SCI to return to meaningful employment following discharge.

- Advocacy of the rights of the disabled (especially those with work-related SCIs) could be done in partnership with non-governmental organisations that fight for the rights of the disabled, tertiary institutions and the state. The social development, health and education cluster of ministries, and Department of Women, Children and People with Disabilities would be especially important in this regard.

- Medical rehabilitation interventions and assistive devices should be made available according to the needs of a particular individual, identified through more focused rehabilitation plans. 
- Referral to community-based rehabilitation structures should be strengthened to ensure continued care to people with SCIs, especially to primary health care facilities close to where the individual lives.

- Structured follow-ups for discharged individuals with SCIs should be established. This could be done telephonically or physically.

- Extensive data can be mined from private rehabilitation facilities. Partnerships should be engaged upon with tertiary institutions, to improve research outputs and create evidence to improve the quality of medical rehabilitation interventions.

- More time should be invested by rehabilitation professionals in on-going support and education to the families of those with and SCI. This should include information about SCI, the impact it has on an individual and managing and coping with the injury.

\subsubsection{Recommendations To Individuals With Work-Related SCI And Their Families}

- Family members, caregivers and individuals with work-related SCI should ensure they are trained, before discharge, in secondary complications and the prevention thereof.

- Individuals should make sure that they comply with rehabilitation interventions with support from NGOs, family, community and health professionals.

- They should make attempts to attend all follow-up appointments.

- They should make sure that they access the social benefits.

- They should ensure that they attend workshops or training that will improve their skills that will enable them to participate in the open labour market. The Department of Labour offers skills training to individuals with disabilities.

- They must locate resources in the community that will enhance their level of participation, thereby improving their quality of life.

- If in doubt about anything relating to their injury, they should seek clarity from rehabilitation professionals, NGOs or government offices.

- Family members or caregivers should make sure that they clearly understand their roles and responsibilities and get adequate skills training to meet expectations and alleviate the burden of care.

\subsubsection{Recommendations To Government, Policy Makers And Business}

- A multi-sectoral and coordinated effort should be made to prevent SCIs in the workplace. The target age group would be $26-45$ and would take place in the urban and peri-urban areas of Gauteng. 
- The Departments of Labour, Transport and of Community Safety, municipal Traffic Management authorities, private sector (Business), and the National Roads Agency should together embark on a road traffic accidents prevention initiative.

- Employers can team up with these agencies to make sure that vehicles are roadworthy and that employees comply with traffic regulations. Companies should try to ensure that deliveries of merchandise are not done during peak traffic times especially on Mondays and Fridays. All company vehicles should be clearly marked to make monitoring easier.

- Businesses should work in partnership with the police to make sure that police are visible on weekends and at night to minimise the risk of robberies which can put the security officers at risk.

- The national Department of Health should establish a National Register or database for all individuals with SCIs. This database would make it easy to draw information on the patterns and causes of SCIs so that specific preventative strategies can be drawn up.

- The Departments of Health (Occupational Health Services) and Labour should embark on Health Promotion in the Workplace preventative strategies by focusing on the training employees about the causes of SCIs. Specific industries to be targeted are service, mining, engineering, business and construction.

- The Departments of Health and Labour should make funds available to ensure that research in this field continues and should make information easily accessible to researchers within the appropriate legislative framework. 


\section{REFERENCES}

Ackery, A., Tator, C. and Krassioukov, A. (2004). A Global Perspective on Spinal Cord Injury Epidemiology. Journal of Neurotrauma, 21(10), 1355-1370.

Akbar-Khanzadeh, F., Bisesi, M. and Rivas, R. (1995). Comfort of Personal Protective Equipment. Applied Ergonomics, 26(3), 195-198.

Alexander, C.J., Hwang, K, and Sipski, M.L. (2002). Mothers with spinal cord injuries: impact on marital, family, and children's adjustment. Archive of Physical Medicine and Rehabilitation, 83(1), 24-30.

Barss P., Djerrari H., Leduc B.E., Lepage Y. and Dionne C.E. (2008). Risk factors and prevention for spinal cord injury from diving in swimming pools and natural sites in Quebec, Canada: A 44-year study. Accident Analysis and Prevention, 40(2), 787-97.

Bennett C.J., Young M.N. and Darrington H. (1995). Differences in urinary tract infections in male and female spinal cord injury patients on intermittent catheterization. Paraplegia, 33(2), 69-72.

Bettger, J.S. and Stineman, M.G. (2007). Effectiveness of Multidisciciplinary Rehabilitation Services in Postacute Care: State-of-the-Science. A review. Archive of Physical Medicine and Rehabilitation, 88(11), 1526-1534.

Bhide, V., Edmonds, V. and Tator, C. (2000). Prevention of spinal cord injuries caused by diving: evalaution of the distribution and usage of diving safety video in high schools. Injury Prevention, 6(2), 154-156.

Bromley, I. (2006). Tetraplegia and Paraplegia. Sixth ed. London: Churchill Livingstone Elsevier.

Burns, S. and Ditunno, J. (2001). Establishing Prognosis and Maximizing Functional Outcomes After Spinal Cord Injury: A Review of Current and Future Directions in Rehabilitation Management. Spine, 26(24 Suppl), 137-145.

Carpenter, C. and Suto, M. (2008). Qualitative Research for Occupational and Physical Therapists: A Practical Guide. Oxford: Blackwell Publishing.

Chabok, S.Y., Safaee, M., Alizadeh, A., Dafchahi, M.A., Taghinnejadi, O. and Koochakinejad, L. (2009). Epidemiology of Traumatic Spinal Injury: A Descriptive Study. Acta Medica Iranica 2010, 48(5), 308-311.

Chan, R. (2000). How does spinal cord injury affect marital relationship? A story from both sides of the couple. Disability and Rehabilitation, 22(17), 764-775.

Chan, S. and Chan, A. (2005). Rehabilitation outcomes following traumatic spinal cord injury in a tertiary spinal cord injury centre: a comparison with the international standard. Spinal Cord, 43(8), 489-498.

Charlifue, S. and Gerhart, K. (2004). Community Integration in Spinal Cord Injury of Long Duration. NeuroRehabilitation, 19(2), 91-101.

Chen, Y., Henson, S., Jackson, A.B. and Richards, J.S. (2006). Obesity intervention in persons with spinal cord injury. Spinal Cord, 44, 82-91. 
Chetty, L.J., Jelsma, J. and Maart,S.P. (2009). How compliant are beverage employees to occupational health and safety regulations? South African Journal of Physiotherapy, 65(1), 28-31.

Cifu, D., Huang, M., Kolakowsky-Hayner, S. and Seel, R. (1999a). Age, Outcome and Rehabilitation Costs after Paraplegia Caused by Traumatic Injury of the Thoracic Spinal Cord, Conus Medullaris and Cauda Equina. Journal of Neurotrauma, 16(9), 805-815.

Cifu, D., Seel, R., Kreutzer, J., Marwitz, J., McKinley, W. and Wisor, D. (1999b). Age, outcome, and rehabilitation costs after tetraplegia spinal cord injury. NeuroRehabilitation, 12, 177-185.

Couto, M.T. Dhaffala, A. and Levy, L.F. (1994). Spinal column and cord injury in Harare Central Hospital. African Journal of Neurological Sciences, 36, 213-218.

Craig, A., Hancock, K., Dickson, H. and Chang, E. (1997). Longterm psychological outcomes in spinal cord injured persons: results of a controlled trial using cognitive behavioural therapy. Archive of Physical Medicine and Rehabilitation, 78(1), 33-38.

DeVivo, M., Biering-Sørensen, F., Charlifue, S., Noonan, V., Post, M., Stripling, T. and Wing, P. (2006). International Spinal Cord Injury Core Data Set. Spinal Cord, 44, 535-540.

DeVivo, M., Hawkins, L.N., Richards, J.S. and Go, B.K. (1995). Outcomes of post-spinal cord injury marriages. Archives of Physical Medical Rehabilitation. 76(2):130-138.

Dijkers, M. (1998). Community integration: conceptual issues and measurement approaches in rehabilitation research. Topics in Spinal Cord Injury Rehabilitation, 4, 1-15.

Divanoglou, A. and Levi, R. (2009). Incidence of traumatic spinal cord injury in Thessaloniki, Greece and Stockholm, Sweden: a prospective population based study. Spinal Cord, 47(9), 796-801.

Domholdt, E. (2005). Rehabilitation Research: Principles and Applications. Third ed. Missouri: Elsevier Saunders.

Dryden, D., Saunders, L., Rowe, B., May, L., Yiannakoulias, N., Svenson, L. and Voaklander, D. (2004). Utilisation of health services following spinal cord injury: a 6-year follow up study. Spinal Cord, 42, 512-525.

Dube A.K. (2005). The role and effectiveness of disability legislation in South Africa. East London: Samaita Consultancy and Programme Design.

Dumont, R., Okwonko, D., Verma, S., Hurlbert, J., Boulos, P., Ellegala, D. and Dumont, A. (2001). Acute Spinal Cord Injury, Part 1: Pathophysiologic Mechanisms. Clinical Neuropharmacology, 24(5), 254-264.

Eastwood, E.A., Hagglund, K.J., Ragnarsson, K.T., Gordon, W.A. and Marion, R.J. (1999). Medical rehabilitation length of stay and outcomes for persons with traumatic spinal cord injury 19901997. Archives of Physical Medicine and Rehabilitation, 80(11),1457-1463.

Ekong, C. and Tator, C. (1985). Spinal Cord Injury in the Work Force. The Canadian Journal of Surgery, 28(2), 165-167.

Emejulu, J.E., Ekweogwu, O.C. and Nottidge, T. (2009). Patterns of spinal injury in a new neurosurgical centre: A 2-year prospective study. East and Central African Journal of Surgery, 14(1), 76-80. 
Feldstein, A., Breen, V. and Dana, N. (1998). Prevention of Work-Related Disability. American Journal of Preventive Medicine, 14(3S), 33-39.

Fiedler, I., Laud, P., Maiman, D. and Apple, D. (1999). Economics of Managed Care in Spinal Cord Injury. Archive of Physical Medicine and Rehabilitation, 80, 1441-1449.

French, D.D., Campbell, R.R., Sabharwal, S., Nelson, A.L., Palacios, P.A., Gavin-Dreschnack, D.

(2007). Health care costs for patients with chronic spinal cord injury in the Veterans Health

Administration. Journal of Spinal Cord Medicine 30(5):477-81.

Frielingsdorf, K. and Dunn, R. (2007). Cervical spine injury outcome - a review of 101 cases treated in a tertiary referral unit. South African Medical Journal, 97(3), 203-207.

Gauteng (South Africa.) Office of the MEC for Community Safety. (2007). Gauteng Safety Strategy, 2006-2014. Crime Quarterly (19), 29-35.

Gershon, R.M., Vlahov, D., Felknor,S.A., Vesley,D., Johnson, P.C., Delcios, G.L. and Murphy,L.R. (1995). Compliance with universal precautions among health care workers at three regional hospitals. American Journal of Infection Control, 23(4), 225-236

Gosselin, R. and Coppotelli, C. (2005). A follow up study of patients with spinal cord injury in Sierra Leone. International Orthopaedics, 29, 330-332.

Griffin, M., Opitz, J., Kurland, L., Ebersold, M. and O'Fallon, W. (1985). Traumatic Spinal Cord Injury In Olmsted County, Minnesota, 1935-1981. Americal Journal of Epidemiology, 121(6), 884-895.

Harcourt, M.W., Wood, G. and Harcourt, S. (2004). Do unions affect employer compliance with the law? New Zealand evidence for age discrimination. British Journal of Industrial Relations, 42, 527-541.

Hardcastle, P. (1987). Towards the prevention of spinal cord injury. Medical Journal of Australia, 149(10), 552-555.

Hart, C. and Williams, E. (1994). Epidemiology of spinal cord injuries: a reflection of changes in South African society. Paraplegia, 32, 709-714.

Harvey, L. (2008). Management of Spinal Cord Injuries: A Guide for Physiotherapists. London: Butterworth Heinemann.

Hawley, C.A., Taylor, R., Hellawell, D.T. and Pentland, B. (1999).Use of the functional assessment measure (FIM+FAM) in head injury rehabilitation: a psychometric analysis. Journal of Neurology, Neurosurgery and Psychiatry, 67, 749-754.

Hermanus, M.A. (2007). Occupational health and safety in mining - status, new developments and concerns. Journal of the South African Institute of Mining and Metallurgy, 107, 531-538.

Hess, R. (2004). Retrospective Studies and Chart Reviews. Respiratory Care, 49(10), 1171-1174.

Higgins, D., Casini, V., Bost, P., Johnson, W. and Rautiainen, R. (2001). The Fatality Assessment and Control Evaluation Programme's Role in the Prevention of Occupational Fatalities. Injury Prevention, 7, 27-33.

Hu, S.L., Lee, C.C., Shiao, J.S.C. and Guo,Y.L. (1998). Employers' awareness and compliance with 
occupational health and safety regulations in Taiwan. Occupational Medicine, 48(1), 17-22.

Igun, G., Obekpa, O., Ugwu, B. and Nwadiaro, H. (1999). Spinal Injuries in the Plateau State, Nigeria. East African Medical Journal, 76(2), 75-79.

Iles, R. and Davidson, M. (2006). Evidence based practice: a survey of physiotherapist's current practice. Physiotherapy Research International, 11(2), 93-103.

International Labour Organization. (1998). World Employment Report 1998/99: Employability in the Global Economy - How Training Matters. Geneva.

Iwegbu, C. (1983). Traumatic Paraplegia in Zaria, Nigeria: The Case for a Centre for Injuries of the Spine. Paraplegia, 21, 81-85.

Jackson, A., Dijkers, M., DeVivo, M. and Pokzatek, R. (2004). A Demographic Profile of New Traumatic Spinal Cord Injuries: Change and Stability Over 30 Years. Archive of Physical Medicine and Rehabilitation, 85, 1740-1748.

Jacobs, B and Jeebhay, M. (n.d.) Occupational Health Services in South Africa. [Online]. Available: ftp://apollo.hst.org.za/pubs/sahr/1999/chapter19.pdf. Accessed, 13 March 2012.

Joubert, DM. (2002). Occupational Health Services Challenges and Success in Developing Countries: A South African perspective. International Journal of Occupational and Environmental Health, $8(2), 119-124$

Joubert, G., Ehrlich, R., Katzenellenbogen J. and Abdool Karim, S. (2007). Epidemiology: a research manual for South Africa. Second ed. Cape Town: Oxford University Press.

Karan-Zebari, D., de Leon, M.B. and Kalpakjian, C.Z. (2011). Predictors of marital longevity after new spinal cord injury. Spinal Cord, 49, 120-124.

Kattail, D., Furlan, J. and Fehlings, M. (2009). Epidemiology and Clinical Outcomes of Acute Spine Trauma and Spinal Cord Injury: Experience From a Specialised Spine Trauma Center in Canada in Comparison with a Large National Registry. The Journal of Trauma: Injury, Infection and Critical Care, 67(5), 936-942.

Kirchberger I., Cieza A., Biering-Sørensen, F., Baumberger, M., Charlifue, S., Post, M.W., Campbell, R., Kovindha, A., Ring, H., Sinnott, A., Kostanjsek, N. and Stucki, G. (2010). ICF Core Sets for individuals with spinal cord injury in the early post-acute context. Spinal Cord, 48(8), 297-304.

Kok, P. and Wentzel, M. (2004). HSRC migration studies: data generated, methodological details, and some highlights. Paper presented at the workshop of the Department of Social Development on the Regional Population and Development Strategy, Pretoria, 2 September.

Krassioukov, A., Eng, J.J., Claxton, G., Sakakibara, B.M. and Shun, S. (2010). Neurogenic bowel management after spinal cord injury: a systematic review of the evidence. Spinal Cord, 48, 718733.

Krause, J.S., Carter, R.E, Pickelsimer, E.E. and Wilson D. (2008). A prospective study of health and risk of mortality after spinal cord injury. Archive of Physical Medicine and Rehabilitation, 89(8), 1482-1491.

Krause, J.S., Kewman, D., DeVivo, M., Maynard, F., Coker, J., Roach, M. and Ducharme, S. (1999). Employment after spinal cord injury: an analysis of cases from the Model Spinal Cord Injury 
Systems. Archive of Physical Medicine and Rehabilitation, 80, 1492-1500.

Krause, J.S., Terza, J.V., Erten, M., Focht, K.L. and Dismuke, C.E. (2012). Predictors of Post-Injury Employment and Percentage of Time Worked after Spinal Cord Injury. Archives of Physical Medicine and Rehabilitation, 93(2), 373-375.

Kroll T., Neri M. and Ho P-S. (2007). Secondary Conditions in Spinal Cord Injury: Results from a Prospective Survey. Disability and Rehabilitation, 29(15), 1229-1237.

Kroll, T., Neri, M., and Miller, K. (2005). Using Mixed Methods in Disability and Rehabilitation Research. Rehabilitation Nursing, 30(3),106-112.

Kwon, B.K., Tetzlaff, W., Grauer, J.N. and Beiner, J. (2004). Pathophysiology and pharmaco-logic treatment of acute spinal cord injury. The SpineJournal, 4, 451-464.

Le Roux, J. and Dunn, R. (2005). Gunshot injuries of the spine - a review of 49 cases managed at the Groote Schuur Acute Spinal Cord Injury Unit. South African Journal of Surgery 43(4), 165-168.

Levy, L., Makarowo, S., Madzivire, D., Bhebhe, E., Verbeek, N. and Parry, O. (1998). Problems, Struggles and Some Success with Spinal Cord Injury in Zimbabwe. Spinal Cord, 36, 213-218.

Lidal, I., Huynh, T. and Biering-Sørensen, F. (2007). Return to work following spinal cord injury: a review. Disability and Rehabilitation, 29(17), 1341-1375.

Life Health Care. (n.d.) [Online] Available: http://www.lifehealthcare.co.za/Hospitals/Acute_Rehabilitation_Services.aspx $)$. Accessed, 12 June 2011.

Maynard, F.M., Bracken, M.B., Creasey, G., Ditunno, J.F., Donovan, W.H., Ducker, T.B. Garber, S.L., Marino, R.J., Stover, S.L., Tator, C.H., Waters, R.L., Wilberger, J.E. and Young,W. (1997). International standards for neurological and functional classification of spinal cord injury. Spinal Cord, 35, 266-274.

McDonald, J. and Sadowsky, C. (2002). Spinal Cord Injury. The Lancet, 359, 417-425.

McDonald, K. (2008). The impact of crime on small business in South Africa. Johannesburg: Small Business Project.

McKinley, W., Jackson, A., Cardenas, D. and DeVivo, M. (1999). Long-Term Medical Compliactions After Traumatic Spinal Cord Injury: A Regional Model Systems Analysis. Archive of Physical Medicine and Rehabilitation, 80, 1402-1410.

McKinley, W.O., Seel, R.T., Gadi, R.K. and Tewksbury, M.A. (2001). Nontraumatic vs. traumatic spinal cord injury. American Journal of Physical Medicine and Rehabilitation, 80, 693-699.

McPherson, D. and McDougall, K.M. (2007). PPE Compliance: Training begins with clear, thorough communication. [ Online] Available: http://www.ishn.com/copyright/BNP_GUID_9-52006_A_10000000000000069531?vie. Accessed, 1 August 2009.

McPherson, K.M., Pentland, B., Cudmore, S.F. and Prescott, R.J. (1996). An inter-rater reliability study of the Functional Assessment Measure (FIM+FAM). Disability Rehabilitation, 18 (7), 341347.

Meade, M.A., Lewis, A., Jackson, M.N. and Hess, D.W. (2004). Race, employment, and spinal cord injury. Archive of Physical Medicine and Rehabilitation, 85(11), 1782-92. 
Migliorini, C., Tonge, B. and Talepos, G. (2008). Spinal cord injury and mental health. Australian and New Zealand Journal of Psychiatry, 42(4), 309-314.

Mohammed, A., Edino, S., Ochicha, O., and Umar, A. (2005). Epidemiology of gunshot injuries in Kano, Nigeria. Nigerian Journal of Surgical Research, 7(3-4), 296-299.

Naidoo, J. and Wills, J. (2009). Foundations for Health Promotion. London: Bailliere Tindal.

Noakes, T.D. (2007). Prevention of spinal cord injuries in rugby union. British Medical Journal 334, $1122-1123$.

Nobunaga, A., Go, B. and Karunas, B. (1999). Recent Demographic and Injury Trends in People Served by the Model Spinal Cord Injury Care Systems. Archive of Physical Medicine and Rehabilitation, 80, 1372-1378.

North, N. (1999). The psychological effects of spinal cord injury: a review. Spinal Cord, 37, 671-679.

O'Hare, P. and Hall, K.M. (1997). Preventing Spinal Cord Injuries through safety education programmes. American Rehabilitation. [Online] Available: http://findarticles.com/p/articles/mi m0842/is n1 v23/ai 19755792/pg 4/?tag=content;col1. Accessed, 12 June 2011.

Obalum, D., Giwa, S., Adekoya-Cole, T. and Enweluzo, G. (2009). Profile of spinal injuires in Lagos, Nigeria. Spinal Cord, 47, 134-137.

O'Connor, P. (2000). Development and Utilisation of the Australian Spinal Cord Injury Register. Spinal Cord, 38, 597-603.

O'Connor, P. (2001). Work-related spinal cord injury, Australia 1986-97. Injury Prevention, 7, 29-34.

O'Connor, P. (2002a). Incidence and patterns of spinal cord in Australia. Accident Analysis and Prevention, 34(4), 405-415.

O'Connor, P. (2002b). Injury to the spinal cord in motor vehicle traffic crashes. Accident Analysis and Prevention, 34, 477-485.

O'Connor, P. (2006a). Trends in Spinal Cord Injury. Accident Analysis and Prevention, 38(5), 71-77.

O'Connor, P. and Brown, D. (2006). Relative risk of spinal cord injury in road crashes involving seriously injured occupants of light passenger vehicles. Accident Analysis and Prevention, 38, 1081-1086.

O'Connor, R. and Murray, P. (2006). Review of spinal cord injuries in Ireland. Spinal Cord, 44(8), 445-448.

Odendaal, T. (1991a). Injuries of the cervical spinal cord: the Garankuwa experience. South African Medical Journal, 80, 75-79.

Odendaal, T. (1991b). Sub-cervical spinal injuries with neural involvement. South African Medical Journal, 80, 219-223.

Olasode, B., Komalafe, I., Komalafe, M. and Olasode, O. (2006). Traumatic spinal cord injuires in Ile-lfe, Nigeria, and its environs. Tropical Doctor, 36, 181-182.

Osterthun, R., Post, M.W. and van Asbeck, F.W. (2009). Characteristics, length of stay and functional 
outcome of patients with spinal cord injury in Dutch and Flemish rehabilitation centres. Spinal Cord. 47(4), 339-44.

Ottomanelli, L. and Lind, L. (2009). Review of Critical Factors Related to Employment After Spinal Cord Injury: Implications for Research and Vocational Services. The Journal of Spinal Cord Medicine, 32(5), 503-531.

Paddison, S. and Middleton, F. (2005). Physical Management in Neurological Rehabilitation. Third ed. Netherlands: Elsevier.

Pagliacci, M., Franceschini, M., Di Clemente, B., Agosti, M. and Spizzichino, L. (2003). A multicentre follow-up of clinical aspects of traumatic spinal cord injury. Spinal Cord, 45, 404410 .

Pagliacci, M.C., Celani, M.G., Spizzichino, L., Zampolini, M., Aito, S., Citterio, A., Finali, G., Loria, D., Ricci, S., Taricco, M. and Franceschini, M. (2003). Spinal cord lesion management in Italy: a 2-year survey. Spinal Cord, 41, 620-628

Pickett, G., Campos-Benitez, M., Keller, J. and Duggal, N. (2006). Epidemiology of Traumatic Spinal Cord Injury. Spine, 31(7), 799-805.

Pickett, W., Simpson, K., Walker, J. and Brison, R. (2003). Traumatic Spinal Cord in Ontario, Canada. The Journal of Trauma: Injury, Infection and Critical Care, 55(6), 1070-1076.

Pless, B. (2000). Preventing spinal cord injuries: is this the best we can do? Canadian Medical Association Journal, 162 (6), 792-793.

Post, M.D., A.J., Angenot, E.L.D., van Asbeck, F.W.A. and van der Woude, L.H.V. (2005). Duration and functional outcome of spinal cord injury rehabilitation in the Netherlands. Journal of Rehabilitation Research and Development, 42(3), 75-86.

Prochaska, JO. and Velken, WF. (1997). The Transtheoretical model of health behaviour change. The American Journal of Health Promotion, 12(1), 38-48

Putzke, J.D, Hicken, B.L, Richards. (2002). Race: predictor versus proxy variable? Outcomes after spinal cord injury. Archive of Physical Medicine and Rehabilitation, 83(11), 1603-1611.

Rabie, F. (2004). Focus Group Interviews and Data Analysis. Nutrition Society, 63, 655-660.

Rahimi-Movaghar, V., Saadat, S., Rasouli, M., Ganji, S., Ghahramani, M., Zarei, M. and Vaccaro, A. (2009). Prevalence of Spinal Cord Injury in Tehran, Iran. The Journal of Spinal Cord Medicine, 32(4), 428-431.

Raine, S., Meadows, L. and Lynch-Ellerington, M. (2009). Bobath Concept: Theory and Clinical Practice in Neurological Rehabilitation. West Sussex: Blackwell Publishing Ltd.

Rauch A., Escorpizo, R., Riddle, D., Eriks-Hoogland, I., Stucki, G. and Cieza, A. (2010). Using a case report of a patient with spinal cord injury to illustrate the application of the International Classification of Functioning, Disability and Health (ICF) during patient management. Physical Therapy, 90(7), 1039-1052.

Rauch, A., Baumberger M, Moise F.G., von Elm, E. and Reinhardt, J.D. (2011). Rehabilitation needs assessment of persons with spinal cord injury following the 2010 earthquake in Haiti: a pilot study using an ICF-based tool. Journal of Rehabilitation Medicine, 43, 969-975. 
Rosenberg, N., Gerhart, K. and Whiteneck, G. (1993). Occupational spinal cord injury: demographic and etiologic differences from non-occupational injuries. Neurology, 43, 1385-1388.

Rosenberg, R.Z., Zirkle, D.L. and Neuwelt, E.A. (2005). Program self-evaluation: the evaluation of an injury prevention foundation. Journal of Neurosurgery, 102, 847-849.

Saari, J. (2001) Success and failures in occupational injury prevention. Injury Prevention, 7, 1-3.

Sand, A., Karlberg, I. and Kreuter, M. (2006). Spinal cord injured persons' conceptions of hospital care, rehabilitation and a new life situatuion. Scandinavian Journal of Occupational Therapy, 13, 183-192.

Saulino, M. (2011). Rehabilitattion of Persons With Spinal Cord Injuires. Medscape Reference. [Online] Available: http://emedicine.medscape.com/article/1265209-overview\#aw2aab6c12. Accessed, 12 June 2011.

Saulino, M.F. and Vacarro, A.R. (2009). Rehabilitation of Persons With Spinal Cord Injuries eMedicine.[Online] Available: http://www.orthopaedicweblinks.com/cgibin/owl/jump.cgi?ID=16970. Accessed, 12 June, 2011.

Schwandt, T.A. (2001). Dictionary of qualitative inquiry. Second ed. Thousand Oaks,CA: Sage.

Sekhon, L.F. and Fehlings, M.G. (2001). Epidemiology, demographics, and pathophysiology of acute spinal cordinjury. Spine, 26(24), 2-12.

Silver, J.R. and Stewart, D. (1994). The prevention of spinal injuries in rugby football. Paraplegia, $32,442-453$

Sinclair, L.L., Lingard, L.A. and Mohabeer, R.N. (2009). What's so great about rehabilitation teams? An ethnoghraphic study of interprofessional collaboration in a rehabilitation unit. Archive of Physical Medicine and Rehabilitation, 90(7), 1196-1201.

Skinner, A. and Turner-Stokes, L. (2006). The use of standardized outcome measures in rehabilitation centres in the UK. Clinical Rehabilitation, 20(7), 609-615.

Smith, G. (2001). Public health approaches to occupational injury prevention: do they work? Injury Prevention, 7, 3-10.

Solagberu, B. (2002). Spinal cord injuries in Ilorin, Nigeria. West African Journal of Medicine, 21(3), 230-232.

Somers, M. (2001). Spinal Cord Injury: Functional Rehabilitation. Second ed. New Jersey: Prentice Hall.

South Africa. (1993). Compensation of Occupational Injuries and Diseases Act 130 of 1993. Pretoria: Government Printer.

South Africa. (1993). Occupational Diseases in Mines and Works Act 208 of 1993. Pretoria: Government Printer.

South Africa. (1993). Occupational Health Safety Act 85 of 1993. Pretoria: Government Printer.

South Africa. (1996). Mine Health Safety Act 29 of 1996. Pretoria: Government Printer. 
South Africa. (2010). Scale of fees for private hospital Gazette 57/58. Pretoria. Government Printer.

South Africa. Office of the Deputy President. (1997). White Paper on an Integrated National Disability Strategy. Pretoria: Rustica Press.

South Africa. Department of Health. (1997). White Paper for the Transformation of the Health System in South Africa.

Statistics South Africa. (2007). Community Survey (2007). Pretoria. [Online] Available: http://www.statssa.gov.za/publications/P0301/P0301.pdf. Accessed, 12 June 2011.

Statistics South Africa. (n.d.) Mid-year Population Estimates 2007. Pretoria. [Online] Available: http://www.statssa.gov.za/publications/P0302/P03022007.pdf. Accessed, 12 April 2009.

Stucki, G. (2005).International Classification of Functioning, Disability, and Health (ICF): a promising framework and classification for rehabilitation medicine. American Journal of Physical Medical and Rehabilitation, 84(10), 733-40.

Thurman, D.J., Burnett, C.L., Beaudoin, D.E., Jeppson, L. and Sniezek, J.E. (1995). Risk factors and mechanisms of occurrence in motor vehicle-related spinal cord injuries: Utah. Accident Analysis and Prevention, 27(3), 411-415.

Tooth, L., McKenna, K. and Geraghty, T. (2003). Rehabilitation outcomes in traumatic spinal cord injury in Australia: functional status, length of stay and discharge setting. Spinal Cord, 41,220230.

Turner-Stokes, L., Nyein, K., Turner-Stokes,T. and Gatehouse, C. (1999). The UK FIM+FAM: development and evaluation. Clinical Rehabilitation, 13(4), 277-287.

Tzannatos, Z. (1998). Women and Labor Market Changes in the Global Economy. World Development, 27, 551-570.

Udosen, A., Ikpeme, A. and Ngim, N. (2007). A Prospective Study of Spinal Cord Injury in the University of Calabar Teaching Hospital, Calabar, Nigeria: A Preliminary Report. The Internet Journal of Orthopaedic Surgery, 5(1), 1-11.

Umphred, D. (2007). Neurological Rehabilitation. Fifth ed. St. Louis, Missouri: Mosby Elsevier.

UNESCO. (2008). The Global Literacy Challenge. [Online] Available

http://unesdoc.unesco.org/images/0016/001631/163170e.pdf. Accessed, 21 June 2011.

Van Niekerk, A., Suffla, S. and Seedat, M. (2008). Crime, Violence and Injury Prevention in South Africa: Data to Action. MRC-UNISA Crime, Violence and Injury Lead Programme, Tygerberg.

Velmahos, G., Degiannis, E., Hart, K., Souter, I. and Saadia, R. (1995). Changing Profiles in Spinal Cord Injuries and Risk Factors Influencing Recovery after Penetrating Injuries. The Journal of Trauma: Injury, Infection and Critical Care, 38(3), 334-337.

Walters, J. (2008). Overview of public transport policy developments in South Africa. Research in Transport Economics, 22, 98-108.

Webster, B., Giunti, G., Young, A., Pransky, G. and Nesathurai, S. (2004). Work-related tetraplegia: cause of injury and annual medical costs. Spinal Cord, 42, 240-247.

Weil, D. (1991). Enforcing OSHA: The role of labor unions. Industrial Relations, 30(1), 20-36. 
Western Cape (South Africa). Department of Health. (2009). Wheelchair Service Delivery Programme. Wheelchair Service Delivery Manual. Basic (professional) Course. Cape Town: Department of Health.

Whiteneck, G., Tate, D. and Charlifue, S. (1999). Predicting Community Reintegration After Spinal Cord Injury From Demographic and Injury Characteristics. Archive of Physical Medicine and Rehabilitation, 80, 1485-1491.

Wilson, B.A. and McLellan, D.L. (1997). Rehabilitation Studies Handbook. Cambridge. Cambridge University Press.

Winslow C., Bode R.K., Felton D., Chen D. and Meyer Jr, P.R. (2002). Impact of Respiratory Complications on Length of Stay and Hospital Costs in Acute Cervical Spine Injury. Chest, 121(5), 1548-1554.

Winter, B. and Pattani, H. (2008). Spinal Cord Injury. Anaesthesia and Intensive Care Medicine, 9(9), 401-403.

Wootton, B.H. (1997). Gender differences in occupational employment. Monthly Labor Review, $15,15-24$.

World Health Organisation. (2008). Guidelines on the provision of manual wheelchairs in lessresourced settings. Geneva: World Health Organization.

World Health Organisation. (2010). International Classification of Diseases $\left(10^{\text {th }}\right.$ Version).[Online] Available: http://www.who.int/classifications/icd/en/. Accessed, 12 June 2011.

Yeo, J. (1993). Prevention of spinal cord injuries in an Australian study (New South Wales). Paraplegia, 31, 759-763.

Yeo, J. and Walsh, J. (1987). Prevention of spinal cord injuries in Australia. Paraplegia, 25, 221-224.

Young, E. and Murhpy, G.C. (2008). Employment status after Spinal Cord Injury (1992-2005): a review with implications for interpretation, evaluation, further research and clinical practice. International Journal of Rehabilitation Research; 32(1),1-11. 


\section{APPENDICES}

\subsection{Appendix A: Data Collection Tool: Information from the Life Rehabilitation}

\section{Centre}

Participant No .................

\section{SECTIONA: DEMOGRAPHIC INFORMATION}

1. Gender

\begin{tabular}{|r|r|}
\hline Male & Female \\
& \\
1 & 2 \\
\hline
\end{tabular}

2. Age

\begin{tabular}{|c|c|c|c|c|c|c|}
\hline$<15$ & $15-25$ & $26-35$ & $36-45$ & $46-55$ & $56-65$ & $65+$ \\
\hline 1 & 2 & 3 & 4 & 5 & 6 & 7 \\
\hline
\end{tabular}

3. Occupation at time of injury 
4. Industry sector

\begin{tabular}{|c|c|c|c|}
\hline Public Sector & Private Sector & Parastatals & Other \\
\hline 1 & 2 & 3 & 4 \\
\hline
\end{tabular}

If other please specify:

5. Type of industry that the person was employed in

\begin{tabular}{|c|c|c|c|c|c|c|c|c|}
\hline $\begin{array}{l}\text { Construc } \\
\text { tion }\end{array}$ & $\begin{array}{l}\text { Manufac } \\
\text { turing }\end{array}$ & Mining & Agriculture & Tourism & Business & $\begin{array}{l}\text { Service } \\
\text { Provision }\end{array}$ & Transport & Other \\
\hline 1 & 2 & 3 & 4 & 5 & 6 & 7 & 8 & 9 \\
\hline
\end{tabular}

If other please specify:

6. Marital Status

\begin{tabular}{|c|c|c|c|c|c|c|}
\hline $\begin{array}{l}\text { Never } \\
\text { married }\end{array}$ & Married & Divorced & Separated & Widow/er & $\begin{array}{l}\text { Co- } \\
\text { habitate }\end{array}$ & Other \\
\hline 1 & 2 & 3 & 4 & 5 & 6 & 7 \\
\hline
\end{tabular}

If other please specify: 


\section{Home Language}

\begin{tabular}{|c|c|c|c|c|c|c|c|c|}
\hline $\begin{array}{l}\text { IsiXhos } \\
\text { a }\end{array}$ & $\begin{array}{l}\text { IsiZul } \\
\text { u }\end{array}$ & $\begin{array}{l}\text { IsiNdebel } \\
\text { e }\end{array}$ & $\begin{array}{l}\text { SeTswan } \\
\text { a }\end{array}$ & $\begin{array}{l}\text { SePed } \\
\text { i }\end{array}$ & $\begin{array}{l}\text { TshiVend } \\
\text { a }\end{array}$ & $\begin{array}{l}\text { XiTsong } \\
\text { a }\end{array}$ & $\begin{array}{l}\text { Englis } \\
\mathrm{h}\end{array}$ & $\begin{array}{l}\text { Afrikaan } \\
\text { s }\end{array}$ \\
\hline 1 & 2 & 3 & 4 & 5 & 6 & 7 & 8 & 9 \\
\hline
\end{tabular}

\section{Population Group}

\begin{tabular}{|c|c|c|c|c|}
\hline Black & White & Coloured & Indian & Other \\
\hline 1 & 2 & 3 & 4 & 5 \\
\hline
\end{tabular}

9. Geographical Location where injury occurred

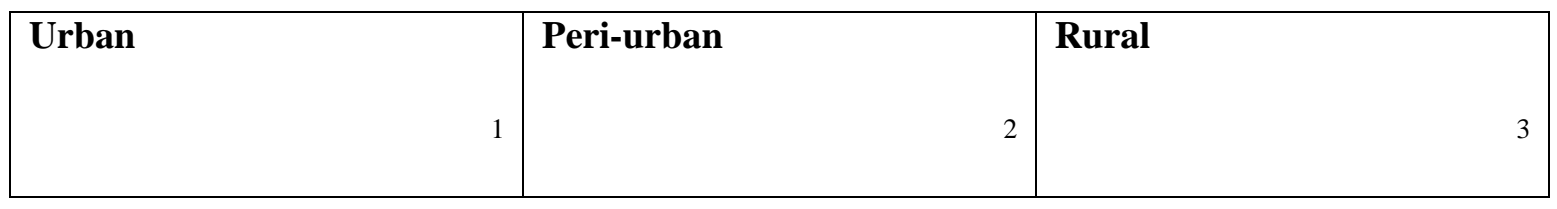

10. Place where accident happened (geographical location) 
SECTION B: DATA RELATED TO THE INJURY

10. Neurological Level

\begin{tabular}{|c|c|}
\hline C1 & 1 \\
\hline C2 & 2 \\
\hline C3 & 3 \\
\hline C4 & 4 \\
\hline C5 & 5 \\
\hline C6 & 6 \\
\hline C7 & 7 \\
\hline C8 & 8 \\
\hline T1 & 9 \\
\hline T2 & 10 \\
\hline T3 & 11 \\
\hline T4 & 12 \\
\hline T5 & 13 \\
\hline T6 & 14 \\
\hline T7 & 15 \\
\hline T8 & 16 \\
\hline T9 & 17 \\
\hline T10 & 18 \\
\hline T11 & 19 \\
\hline
\end{tabular}




\begin{tabular}{|l|r|}
\hline T12 & 20 \\
\hline L1 & 21 \\
\hline L2 & 22 \\
\hline L3 & 23 \\
\hline L4 & 24 \\
\hline L5 & 25 \\
\hline S1 & 26 \\
\hline S2 & 27 \\
\hline S3 & 28 \\
\hline S4 & 29 \\
\hline S5 & 30 \\
\hline
\end{tabular}

11. Classification of injury (According to the ASIA scale)

\begin{tabular}{|c|c|c|c|}
\hline Complete & & Incomplete & \\
\hline ASIA A & ASIA B & ASIA C & ASIA D \\
\hline 1 & 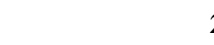 & 3 & 4 \\
\hline
\end{tabular}

12. Date of injury: 
13. Time of injury

\begin{tabular}{|c|c|c|c|}
\hline 06:01 - 12:00 & 12:01-18:00 & 18:01 - 24:00 & 00:01 - 06:00 \\
\hline 1 & 2 & 3 & 4 \\
\hline
\end{tabular}

14. Day of injury

\begin{tabular}{|c|c|c|c|c|c|c|}
\hline Monday & Tuesday & Wednesday & Thursday & Friday & Saturday & Sunday \\
\hline 1 & 2 & 3 & 4 & 5 & 6 & 7 \\
\hline
\end{tabular}

15. Cause of injury

\begin{tabular}{|c|c|c|c|c|}
\hline MVA & Violence & $\begin{array}{l}\text { Train } \\
\text { accident }\end{array}$ & Sport & Miscellaneous \\
\hline 1 & 2 & 3 & 4 & 5 \\
\hline
\end{tabular}

16. If MVA,

\begin{tabular}{|c|c|c|c|c|c|}
\hline \multicolumn{2}{|c|}{ Motor Vehicle } & \multicolumn{2}{|c|}{ Motor Bike } & \multirow{2}{*}{$\begin{array}{l}\text { Pedestrian } \\
\\
3\end{array}$} & \multirow[t]{2}{*}{ Other } \\
\hline Driver & Passenger & Driver & Passenger & & \\
\hline 1 & $\overline{2}$ & 1 & 2 & & \\
\hline
\end{tabular}

If other please specify: 
17. if motor vehicle or motor bike accident crash type?

\begin{tabular}{|l|l|l|l|l|l|}
\hline Rollover & $\begin{array}{l}\text { Collision with another } \\
\text { vehicle }\end{array}$ & $\begin{array}{l}\text { Collision with a } \\
\text { roadside hazard }\end{array}$ & $\begin{array}{l}\text { Loss of } \\
\text { control }\end{array}$ & Other \\
& 2 & 3 & 4 & 5 \\
\hline
\end{tabular}

If other please specify:

18. Violence

\begin{tabular}{|ll|l|l|l|}
\hline Gunshot & Assault & Stabbing & Other \\
& & 2 & 3 & \\
\hline
\end{tabular}

If other please specify:

19 Miscellaneous

\begin{tabular}{|l|l|l|l|l|}
\hline Falls & Hit by a fallen object & Struck by an object & $\begin{array}{l}\text { Lifting or } \\
\text { twisting }\end{array}$ & Other \\
& & & & \\
1 & 2 & 3 & & 5 \\
\hline
\end{tabular}

If other please specify: 
20. Description of activity when injury was sustained

\section{Circumstances around the injury}

\begin{tabular}{|lr|l|l|}
\hline $\begin{array}{l}\text { Employee } \\
\text { negligence }\end{array}$ & & $\begin{array}{l}\text { Employer } \\
\text { negligence }\end{array}$ & Other \\
& 1 & & 2 \\
\end{tabular}

If other please specify: 
22. Employee Negligence

\begin{tabular}{|l|l|l|l|l|r|}
\hline $\begin{array}{l}\text { Substance } \\
\text { Abuse }\end{array}$ & $\begin{array}{l}\text { Not wearing } \\
\text { PPE }\end{array}$ & Speeding & $\begin{array}{l}\text { Not wearing } \\
\text { safety belt }\end{array}$ & $\begin{array}{l}\text { Not following safety } \\
\text { precautions }\end{array}$ & Other \\
\hline 1 & & 3 & & & \\
\hline
\end{tabular}

If other please specify:

23. Employer negligence

\begin{tabular}{|lr|l|l|}
\hline NO PPE & No Policies (SHE) & Other & 3 \\
& & 2 & \\
\hline
\end{tabular}

If other please specify:

24. Date of Admission to Rehabilitation Facility

25. Date of Discharge at the Rehabilitation Facility 
26. Length of stay in Rehabilitation Facility

\begin{tabular}{|l|l|}
\hline & \\
\hline No in Days & \\
\hline
\end{tabular}

27. Additional Injuries sustained during the incident

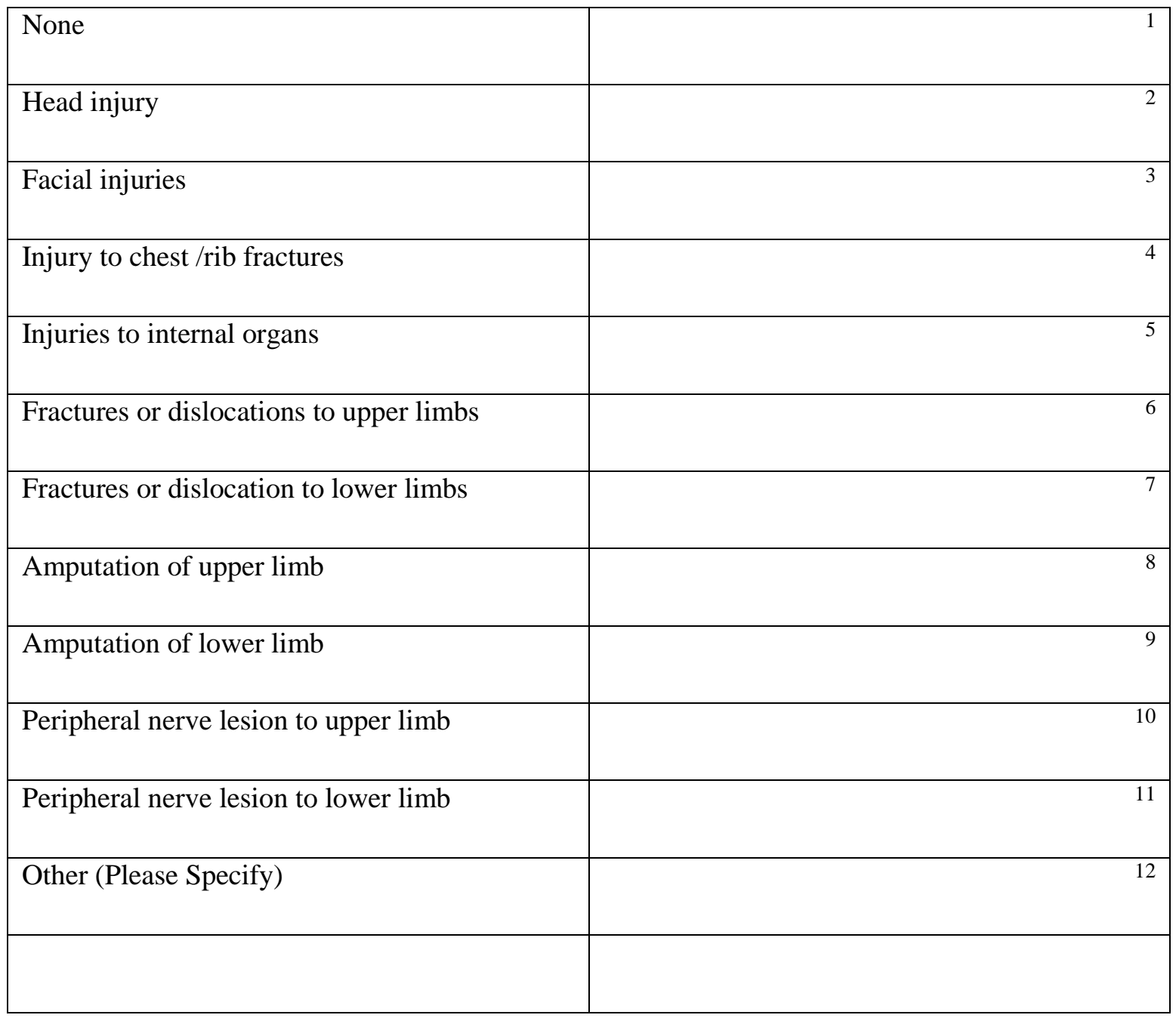




\section{Complications during rehabilitation admission}

\begin{tabular}{|l|r|}
\hline None & 1 \\
\hline Respiratory & 2 \\
\hline Cardiac & 3 \\
\hline Deep vein thrombosis & 5 \\
\hline Organ failure & 6 \\
\hline Bladder infection & 7 \\
\hline Pressure sore & 8 \\
\hline Other (Please Specify & \\
\hline & \\
\hline
\end{tabular}

\section{Rehabilitation: Service Providers Involved}

\begin{tabular}{|l|}
\hline Orthopaedic Surgeon \\
\hline 1 \\
\hline Neurosurgeon \\
\hline Physician \\
\hline 3 \\
\hline Radiologist \\
\hline 4 \\
\hline
\end{tabular}




\begin{tabular}{|c|}
\hline $\begin{array}{l}\text { Pathologists } \\
5\end{array}$ \\
\hline $\begin{array}{l}\text { Pharmacist } \\
6\end{array}$ \\
\hline $\begin{array}{l}\text { Neurologist } \\
7\end{array}$ \\
\hline $\begin{array}{l}\text { Urologist } \\
8\end{array}$ \\
\hline $\begin{array}{l}\text { General Practitioner } \\
9\end{array}$ \\
\hline $\begin{array}{l}\text { Rehabilitation Specialist } \\
10\end{array}$ \\
\hline $\begin{array}{l}\text { Physiotherapist } \\
11\end{array}$ \\
\hline $\begin{array}{l}\text { Occupational Therapist } \\
12\end{array}$ \\
\hline $\begin{array}{l}\text { Social Worker } \\
13\end{array}$ \\
\hline $\begin{array}{l}\text { Psychologist } \\
14\end{array}$ \\
\hline $\begin{array}{l}\text { Prosthesis and Orthotist } \\
15\end{array}$ \\
\hline $\begin{array}{l}\text { Dietician } \\
16\end{array}$ \\
\hline $\begin{array}{l}\text { Other } \\
17\end{array}$ \\
\hline
\end{tabular}




\section{Type of Rehabilitation Services Provided}

\begin{tabular}{|l|l|l|l|}
\hline & Yes & No & Service Provider (s) \\
\hline A family centred approach & & & \\
\hline Individual therapy & & & \\
\hline Group therapy & & & \\
\hline Physical therapy & & & \\
\hline Vocational Rehabilitation & & & \\
\hline Cognitive Behavioural Therapy & & & \\
\hline Diet Therapy & & & \\
\hline
\end{tabular}

\section{Nature of Physical Therapy}

\begin{tabular}{|l|l|l|}
\hline & Yes & No \\
\hline Chest Physiotherapy & & \\
\hline Gait Rehabilitation & & \\
\hline Strengthening Exercises & & \\
\hline Balance Re-education & & \\
\hline Pressure Care & & \\
\hline Passive Movements & & \\
\hline Mat Activities & & \\
\hline Vasomotor Control & & \\
\hline Self Catheterisation & & \\
\hline
\end{tabular}


$\underline{\text { 32. Outcomes of Rehabilitation }}$

\begin{tabular}{|l|l|l|l|}
\hline \multicolumn{2}{|l|}{ Score on Admission } & \multicolumn{2}{l|}{ Score on Discharge } \\
\hline FIM & FAM & FIM & FAM \\
\hline & & & \\
\hline
\end{tabular}

\section{SECTION C: MEDICAL COSTS}

33. Costs of Admission to a rehabilitation facility

\begin{tabular}{|l|l|}
\hline 1998 & 2006 \\
\hline 1 & 9 \\
\hline 2999 & 2007 \\
\hline 2000 & 10 \\
\hline 3 & 2008 \\
\hline 2001 & 11 \\
\hline 4 & 2009 \\
\hline 2002 & 12 \\
\hline 5 & \\
\hline 2003 & \\
\hline 6 & \\
\hline 2004 & \\
\hline 7 & \\
\hline 2005 & \\
\hline 8 & \\
\hline
\end{tabular}


34. Assistive Devices issued

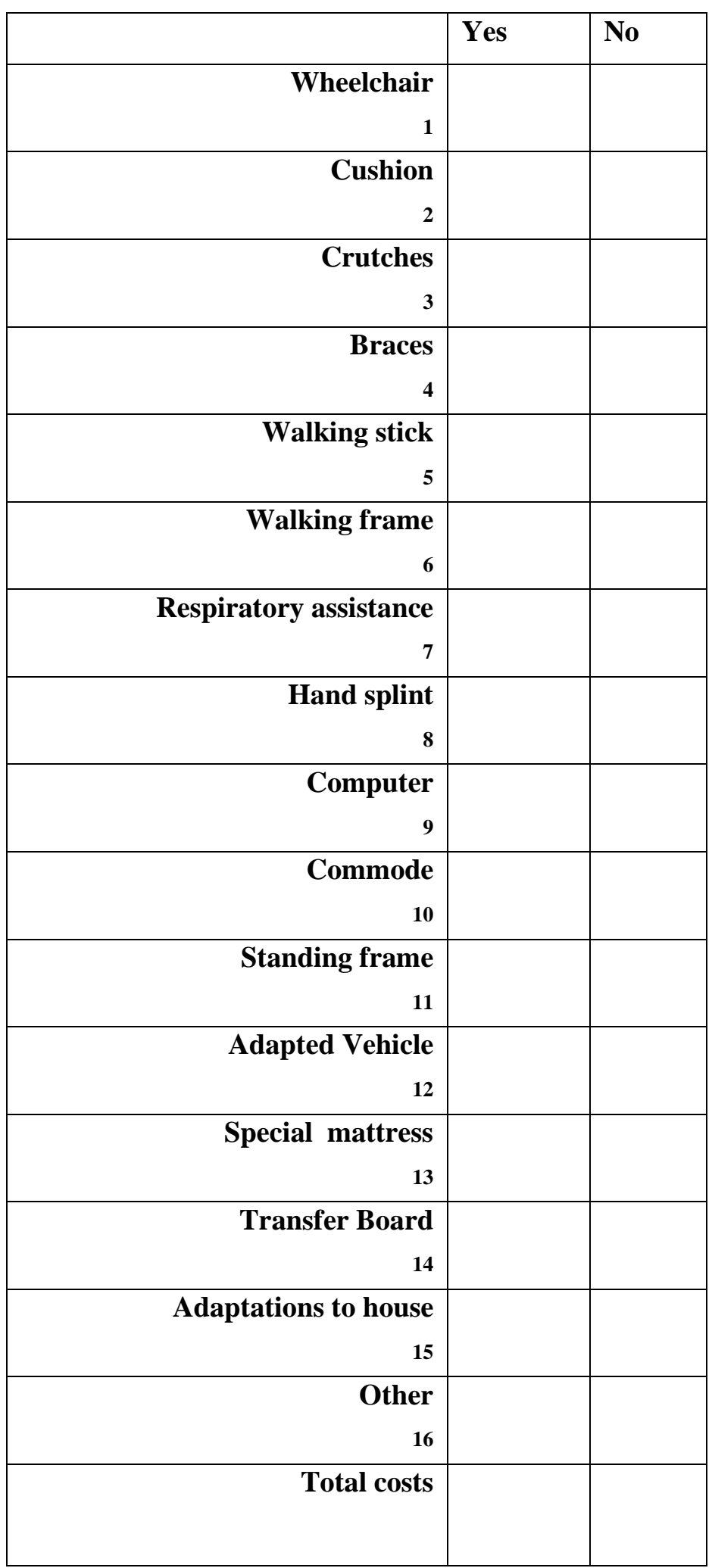

If other please specify: 
SECTION D: POST DISCHARGE INFORMATION

35. Place where the patient is discharged to:

urban

rural

Peri-urban

36. Primary Health Care Services: Availability

\begin{tabular}{|l|l|}
\hline$\underline{\text { Yes }}$ & $\underline{\text { No }}$ \\
\hline $\mathbf{1}$ & $\mathbf{2}$ \\
\hline
\end{tabular}

37. Service providers who provided follow up post discharge and type of follow up

\begin{tabular}{|l|l|l|}
\hline Service Provider & Nature of subsequent visits & $\begin{array}{l}\text { No of visits post } \\
\text { discharge }\end{array}$ \\
\hline $\begin{array}{l}\text { Orthopaedic } \\
\text { Surgeon }\end{array}$ & & \\
\hline Neurosurgeon & & \\
\hline Physician & & \\
\hline Radiologist & & \\
& & \\
\hline Pathologists & & \\
& & \\
\end{tabular}




\begin{tabular}{|l|l|l|}
\hline Pharmacy & & \\
\hline Neurologist & & \\
\hline Urologist & & \\
\hline Specialist & & \\
\hline Physiotherapist & & \\
\hline Orosthesis and & & \\
\hline Social Worker & & \\
\hline Osychologist & & \\
\hline Occupational & & \\
\hline CBR programme & & \\
\hline
\end{tabular}




\section{Section D}

ICF scores in selected domains most often affected by SCI, at discharge, as deducted from information in folder

Scoring:

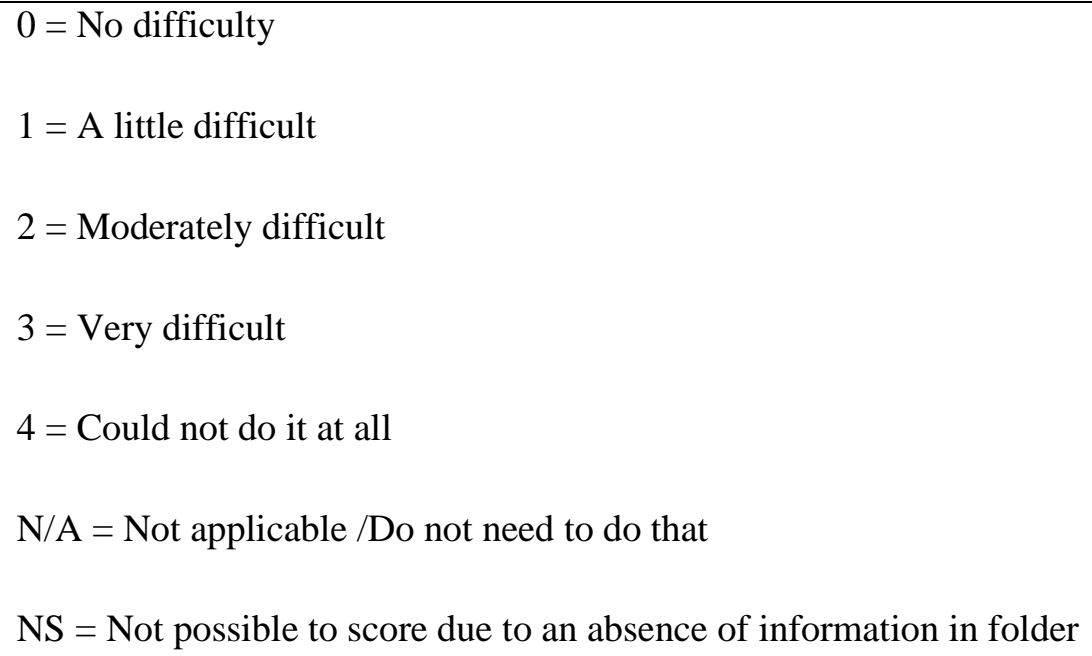

\begin{tabular}{|l|l|l|}
\hline \multicolumn{2}{|c|}{ Communication } & Score \\
\hline 38 & Expressing self & \\
\hline 39 & Starting and maintaining a conversation & \\
\hline 40 & Conversing using devices (Telephone, Computer) & \\
\hline 41 & Reading, writing and calculating & \\
\hline 42 & Lifting and moving /carrying objects & \\
\hline 43 & Fine hand use, grasping, picking up small stuff, doing buttons etc. & \\
\hline 44 & Standing up from sitting & \\
\hline 45 & Walking & \\
\hline
\end{tabular}




\begin{tabular}{|l|l|l|}
\hline 46 & Getting around in wheelchair /other device & \\
\hline 47 & Moving inside the house & \\
\hline 48 & Getting out of the house & \\
\hline 49 & Moving around outside the house in the yard & \\
\hline 50 & Walking /wheeling for a long distance $-1 \mathrm{~km}$ or more & \\
\hline 51 & Doing transfers & \\
\hline 52 & Using transport (Car, taxi, donkey cart) & \\
\hline 53 & Driving (Car, bicycle, horse) & \\
\hline
\end{tabular}

\begin{tabular}{|l|l|l|}
\hline \multicolumn{2}{|l|}{ Self care } & \\
\hline 54 & Washing & \\
\hline 55 & Getting dressed & \\
\hline 56 & Eating & \\
\hline 57 & Drinking & \\
\hline 58 & Using the toilet & \\
\hline 59 & Grooming (Hair. Nails, Beard) & \\
\hline 60 & Looking after own health & \\
\hline 61 & Staying alone for a few days & \\
\hline & Domestic life &
\end{tabular}

\begin{tabular}{|l|l|l|}
\hline 62 & Doing shopping and accessing services & \\
\hline 63 & Preparing meals & \\
\hline 64 & Doing chores in and around the house & \\
\hline 65 & Assisting others & \\
\hline
\end{tabular}

\section{Community integration}




\begin{tabular}{|l|l|l|}
\hline \multicolumn{2}{|l|}{$(164)$} & \\
\hline 66 & Participating in social activities & \\
\hline 67 & Participating in religious activities & \\
\hline 68 & Participating in sport & \\
\hline 69 & Manage own finances & \\
\hline 70 & Participating in politics and citizenship & \\
\hline 71 & Productive activity & \\
\hline 72 & Economically self sufficient & \\
\hline
\end{tabular}

\section{Employment status}

\begin{tabular}{|l|l|l|l|l|}
\hline Unemployed & $\begin{array}{l}\text { Employed by } \\
\text { previous employer }\end{array}$ & $\begin{array}{l}\text { Employed by another } \\
\text { employer }\end{array}$ & Self employed & Other \\
\hline & & & & \\
\hline
\end{tabular}

If other please specify:

78. Mortality Information:

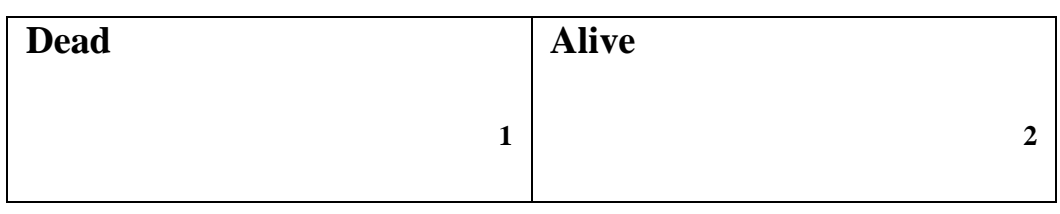




\subsection{Appendix B: Interview Schedule: Employer}

Participant number:

Section A: Demographics Information

1. Gender

\begin{tabular}{|l|l|}
\hline Male & Female \\
\hline 1 & 2 \\
\hline
\end{tabular}

2. Age

\begin{tabular}{|l|l|l|l|l|l|l|}
\hline$<15$ & $15-25$ & $26-35$ & $36-45$ & $46-55$ & $56-65$ & $65+$ \\
\hline & 1 & 2 & 3 & 4 & 5 & 6 \\
\hline
\end{tabular}

\section{Occupation}

4. Name of the Company 
6. Role

\begin{tabular}{|l|l|l|l|l|l|}
\hline Manager & SHE rep & $\begin{array}{l}\text { SHE co- } \\
\text { ordinator }\end{array}$ & $\begin{array}{l}\text { Occupational } \\
\text { Hygenist }\end{array}$ & Supervisor & Other \\
\hline 1 & 2 & & & 3 & 4 \\
\hline
\end{tabular}

\section{Industry sector}

\begin{tabular}{|l|l|l|l|}
\hline Public Sector & Private Sector & Parastatals & Other \\
\hline 1 & 2 & 3 & 4 \\
\hline
\end{tabular}

\section{Company Sector}

\begin{tabular}{|l|l|l|l|}
\hline Agriculture & Service & Manufacturing & Other \\
\hline 1 & 2 & 3 & 4 \\
\hline
\end{tabular}

9. Marital Status

\begin{tabular}{|l|l|l|l|l|}
\hline Married & Divorced & Separated & Widow/er & Never Married \\
\hline 1 & 2 & 3 & 4 & 5 \\
\hline
\end{tabular}




\begin{tabular}{|l|l|l|l|}
\hline $\begin{array}{l}\text { No formal } \\
\text { education }\end{array}$ & Primary & Secondary & Tertiary \\
\hline 1 & 2 & 3 & 4 \\
\hline
\end{tabular}

11. Years of Work Experience (in this company)

\begin{tabular}{|l|l|l|l|l|}
\hline $0-5$ & $6-10$ & $11-15$ & $16-20$ & $20+$ \\
\hline 1 & 2 & 3 & 4 & 5 \\
\hline
\end{tabular}

12. Occupational Health and Safety Training

\begin{tabular}{|l|l|}
\hline Yes & No \\
\hline 1 & 2 \\
\hline
\end{tabular}

Interview Schedule:

1. What is your role in the company with regards to occupational health and safety?

2. What safety precautions and policies are followed at this company?

3. Do these policies adhere to Occupational Safety and Health Act (OSHA) regulations?

4. What do you understand under OSHA?

5. What is COIDA?

6. Who is responsible to implement these policies? 
7. Tell me about employee compliance with the health and safety rules?

8. What measures are in place to ensure that employees are compliant?

9. What free protective equipment does the company offer employees?

10. How compliant are employees with wearing these?

11. To what extent, was employees' part of the process of drafting the health and safety policy in your company?

12. Do you think this is a safe company to work for? Please explain your answer.

13. What suggestions do you have for things that can be done to improve health and safety of workers in this company?

14. Is there anything else that you want to share with me on this topic?

I will contact you again, if I need clarity on the issues we have discussed. Thank you 


\subsection{Appendix C: Interview Schedule: Employee}

Participant Research Number: ......

Section A: Demographics Information

1. Gender

\begin{tabular}{|l|l|}
\hline Male & Female \\
\hline 1 & 2 \\
\hline
\end{tabular}

2. Age

\begin{tabular}{|l|l|l|l|l|l|l|}
\hline$<15$ & $15-25$ & $26-35$ & $36-45$ & $46-55$ & $56-65$ & $65+$ \\
\hline & 1 & 2 & 3 & 4 & 5 & 6 \\
\hline
\end{tabular}

\section{Occupation}

4. Name of the Company 
5. Position in company

6. Industry sector

\begin{tabular}{|l|l|l|l|}
\hline Public Sector & Private Sector & Parastatals & Other \\
\hline 1 & 2 & 3 & 4 \\
\hline
\end{tabular}

7. Company Sector Give all options

\begin{tabular}{|l|l|l|l|}
\hline Agriculture & Service & Manufacturing & Other \\
\hline 1 & 2 & 3 & 4 \\
\hline
\end{tabular}

8. Marital Status

\begin{tabular}{|l|l|l|l|l|}
\hline Married & Divorced & Separated & Widow/er & Never Married \\
\hline 1 & 2 & 3 & 4 & 5 \\
\hline
\end{tabular}

9. Level of Education

\begin{tabular}{|l|l|l|l|}
\hline $\begin{array}{l}\text { No formal } \\
\text { education }\end{array}$ & Primary & Secondary & Tertiary \\
\hline 1 & 2 & 3 & 4 \\
\hline
\end{tabular}


10. Years of Work Experience (in this company)

\begin{tabular}{|l|l|l|l|l|}
\hline $0-5$ & $6-10$ & $11-15$ & $16-20$ & $20+$ \\
\hline 1 & 2 & 3 & 4 & 5 \\
\hline
\end{tabular}

\section{Occupational Health and Safety Training}

\begin{tabular}{|l|l|}
\hline Yes & No \\
\hline 1 & 2 \\
\hline
\end{tabular}

\section{Interview Schedule:}

1. Tell me what you know about the OSHA Act?

2. Tell me about the compensation fund?

3. Tell me about the policies at work that deal with health and safety issues?

4. Do your work activities put you at risk of sustaining a spinal cord injury?

5. Who is the Safety and Health Rep at work? And what do they do?

6. What are the procedures that must be followed to prevent injuries in the work place?

7. How do you protect yourself at work whilst performing your duties? 
8. Name the protective equipment used to prevent injuries at work?

9. Do you have to pay for the protection equipment at work?

9. How do you feel about wearing protective Equipment at work?

10. How frequently do you were your protective equipment?

11. Do you feel safe at work? Explain why?

12. What type of injuries have you sustained or seen fellow workers sustained at this place of work?

13. What can be done to prevent these injuries?

14. In your opinion how much support does the company give to injured workers?

12. Is there anything else that you want to share with me on this topic?

Please note that I will contact you if I need more clarity on issues discussed.

Thank you for participating. 
1.4 Appendix D: Interview Schedule for persons who sustained Spinal Cord Injuries

Participant Research Number:

$\underline{\text { Section A: Demographics Information }}$

1. Gender

\begin{tabular}{|l|l|}
\hline Male & Female \\
\hline 1 & 2 \\
\hline
\end{tabular}

2. Age

\begin{tabular}{|l|l|l|l|l|l|l|}
\hline$<15$ & $15-25$ & $26-35$ & $36-45$ & $46-55$ & $56-65$ & $65+$ \\
\hline & 1 & 2 & 3 & 4 & 5 & 6 \\
\hline
\end{tabular}

3. Occupation before the injury

4. Name of the Company 
5. Position in company

6. Industry sector

\begin{tabular}{|l|l|l|l|}
\hline Public Sector & Private Sector & Parastatals & Other \\
\hline 1 & 2 & 3 & 4 \\
\hline
\end{tabular}

\section{Company Sector}

\begin{tabular}{|l|l|l|l|l|}
\hline Agriculture & Service & Manufacturing & Other & \\
\hline $\mathbf{1}$ & $\mathbf{2}$ & $\mathbf{3}$ & $\mathbf{4}$ & \\
\hline
\end{tabular}

8. Marital Status

\begin{tabular}{|l|l|l|l|l|}
\hline Married & Divorced & Separated & Widow/er & Never Married \\
\hline 1 & 2 & 3 & 4 & 5 \\
\hline
\end{tabular}

9. Level of Education

\begin{tabular}{|l|l|l|l|}
\hline $\begin{array}{l}\text { No formal } \\
\text { education }\end{array}$ & Primary & Secondary & Tertiary \\
\hline 1 & 2 & 3 & 4 \\
\hline
\end{tabular}


10. Years of Work Experience (in this company)

\begin{tabular}{|l|l|l|l|l|}
\hline $0-5$ & $6-10$ & $11-15$ & $16-20$ & $20+$ \\
\hline 1 & 2 & 3 & 4 & 5 \\
\hline
\end{tabular}

\section{Occupational Health and Safety Training}

\begin{tabular}{|l|l|}
\hline Yes & No \\
\hline 1 & 2 \\
\hline
\end{tabular}

\section{Current Occupational Status}

\begin{tabular}{|l|l|l|l|}
\hline Employed & Unemployed & Self Employed & Other (please specify) \\
\hline 1 & 2 & 3 & 4 \\
\hline
\end{tabular}

Interview Schedule:

1. Tell me what you know about the OSHA Act?

2. Tell me about the compensation fund?

3. Tell me about the policies at the place of work where you sustained your injury that deal with health and safety issues?

4. Do your work activities put you at risk of sustaining a spinal cord injury? 
5. How much support did the company give you since you sustained your injury?

6. Is there anything else that you want to share with me on this topic?

7. Tell me about your injury?

8. What caused your injury?

9. What could you have done to prevent the injury?

10. What could the employer have done to prevent the injury?

11. Tell me about the process for application for compensation?

12. Are you re-employed? Tell me why /why not?

13. If a policy document would be drawn up to prevent spinal cord injuries, what would you like included in the policy?

Will it be possible if I contact you, should I need clarity or more information about what we have discussed today?

Thank you for participating. 


\subsection{Appendix E: Participant Information Leaflet And Consent Form}

\section{TITLE OF THE RESEARCH PROJECT:}

Work-related Spinal Cord Injuries in Gauteng Province, South Africa

PRINCIPAL INVESTIGATOR: Mr. Ntsikelelo Pefile

ADDRESS: Postnet Suite 409 Private Bag x3 Northriding 2162

CONTACT NUMBERS: 0827228503 (c ) OR 0312607181 (w)

You are being invited to take part in a research project. Please take some time to read the information presented here, which will explain the details of this project. Please ask the researcher any questions about any part of this project that you do not fully understand. It is very important that you are fully satisfied that you clearly understand what this research entails and how you could be involved. Also, your participation is entirely voluntary and you are free to decline to participate. If you say no, this will not affect you negatively in any way whatsoever. You are also free to withdraw from the study at any point, even if you do agree to take part.

This study has been approved by the Committee for Human Research at Stellenbosch University and will be conducted according to the ethical guidelines and principles of the international Declaration of Helsinki, South African Guidelines for Good Clinical Practice and the Medical Research Council (MRC) Ethical Guidelines for Research.

\section{What is this research study all about?}

$>$ The study will be done in various industries or companies in Gauteng Province, South Africa

$>$ This study aims to gather relevant information about people who sustained spinal cord injuries at work and to gather information about compliance of employees and employers regarding rules and regulations of the Occupational Safety and Health Act,1993. This 
information will help the researcher to create guidelines that will assist the employers and employees to prevent spinal cord injuries in the workplace.

\section{Why have you been invited to participate?}

Your participation in this study will assist the researcher to access the information required to fulfill the above-mentioned intentions. During the interview, the researcher will ask you questions that will respond to the aims mentioned above.

\section{What will your responsibilities be?}

$>$ To answer all questions to the best of your ability

\section{Will you benefit from taking part in this research?}

This descriptive study will yield information to be used in the development and formulation of a preventative framework for work-related spinal cord injuries in the study setting i.e. Gauteng Province, South Africa. Lessons learned in this case study can also be, with consideration of demographics, applied to other provinces in South Africa and to all industrial sectors. The study will inform the Department of Labour about the compliance of industries with the Occupational Health and Safety Act policies and regulations in Gauteng Province. This could develop an interest to do more research on this and related topics in other provinces in South Africa to ensure compliance to the Occupational Health and Safety Act 85 of 1993.

\section{Are there risks involved in your taking part in this research?}

No risks are anticipated by the researcher

\section{If you do not agree to take part, what alternatives do you have?}

No alternatives are available. Participation in this study is completely voluntary.

Who will have access to your medical records? 
Only the researcher will have access to the information gathered during the interview and or during the perusal of the medical records. The information will be stored in a store and lock facility and only the researcher will have access to the information. The information will be published in a thesis at the end of the study but all responses will be kept confidential and anonymous.

\section{What will happen in the unlikely event of some form injury occurring as a direct result of your taking part in this research study?}

$>$ No risk of injury is anticipated by participating in this study. Hence no insurance cover is available to the participants

\section{Will you be paid to take part in this study and are there any costs involved?}

No, you will not be paid to take part in the study but your transport and meal costs will be covered for each study visit. There will be no costs involved for you, if you do take part.

\section{Is there any thing else that you should know or do?}

You can contact the Committee for Human Research at 021-938 9207 if you have any concerns or complaints that have not been adequately addressed by your researcher.

$>$ You will receive a copy of this information and consent form for your own records.

\section{Declaration by participant}

By signing below, I agree to take part in a research study entitled (Work-related Spinal Cord Injuries in Gauteng Province, South Africa).

I declare that:

- I have read or had read to me this information and consent form and it is written in a language with which I am fluent and comfortable.

- I have had a chance to ask questions and all my questions have been adequately answered.

- I understand that taking part in this study is voluntary and I have not been pressurised to take part.

- I may choose to leave the study at any time and will not be penalised or prejudiced in any way.

- I may be asked to leave the study before it has finished, if the researcher feels it is in my best interests, or if I do not follow the study plan, as agreed to. 


\section{Declaration by investigator}

I Ntsikelelo Pefile declare that:

- I explained the information in this document to

- I encouraged him/her to ask questions and took adequate time to answer them.

- I am satisfied that he/she adequately understands all aspects of the research, as discussed above

- I did/did not use an interpreter. (If a interpreter is used then the interpreter must sign the declaration below. 


\section{Declaration by interpreter}

I (name) declare that:

- I assisted the investigator Ntsikelelo Pefile to explain the information in this document to (name of participant) using the language medium of Afrikaans/Xhosa/Zulu/SeTswana/TshiVenda/SePedi/IsiSwati/XiTsonga.

- We encouraged him/her to ask questions and took adequate time to answer them.

- I conveyed a factually correct version of what was related to me.

- I am satisfied that the participant fully understands the content of this informed consent document and has had all his/her question satisfactorily answered. 


\subsection{Appendix F: Application for Access to Claimant's Files}

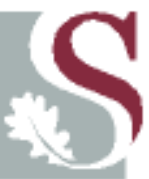

UNIVERSITEIT.STELLENBOSCH.UNIVERSITY

jou kennisvennoot - your knowledge partner

Centre for Rehabilitation Studies

Tygerberg Campus

University of Stellenbosch

The Rehabilitation Standards Manager

Life Health Group

Durban

Dear Mrs. Strydom

\section{Re: Application for Access to Claimant's Files}

I hereby apply for permission to access files (electronic or hard files) of all patients who have sustained spinal cord injury at work for research purposes, for the period between 1998 and 2008. I am a student at the above mentioned department, reading for my Msc (Medical Science) (Rehabilitation). As part of my studies I have to embark on a study that will demonstrate my ability to engage in meaningful research that will provide valuable information that will assist in the prevention of spinal cord injuries in the workplace. Your institution is the only institution with this valuable information readily available. The aim and objectives of the study are as follows: 
Aim

To determine the epidemiology of work-related spinal cord injuries in Gauteng Province, South Africa, and to describe the compliance of employees and employers with the rules and regulations of the Occupational Health and Safety Act of 1993 in order to provide employers and policy makers with the necessary information to develop preventative strategies in the workplace.

\section{Objectives}

- To describe the demographic profile of people who suffered work-related spinal cord injuries in Gauteng Province, South Africa between 01 January 1998 and 31 December 2008.

- To determine the causes and mechanisms of these work-related spinal cord injuries.

- To determine the type and severity of the spinal cord injuries

- To determine the distribution of work-related spinal cord injury in Gauteng with regards to geographical location and industry sectors.

- To determine the incidence of work-related spinal cord injury in Gauteng Province, South Africa between 01 January 1998 and 31 December 2008.

- To determine the costs of medical, surgical and rehabilitation intervention in work-related spinal cord injuries during the study period.

- To determine the attitudes and practices of employees and employers regarding safety and injury prevention issues in each of the identified industries.

- To determine the compliance of various industries to the Occupational Health and Safety Act of South Africa (OSHA), 1993.

- To identify the existing preventative measures in the various industries.

- To develop a preventative framework for work-related spinal cord injuries in the study setting.

The information gathered during the interview and from folders will be kept with utmost confidentiality. Only the researcher and research assistants will have access to the information for data analysis purposes. There will no names or other identifying particulars on the data collection tools. A participant number will be allocated to each participant and each folder. Only these numbers will be used on data collection tools. The identifying particulars of participants and folders will be stored in a separate password protected computer file. All information from the files perused will be kept in strictest confidentiality. No names or any information that can divulge the identity of the participant 


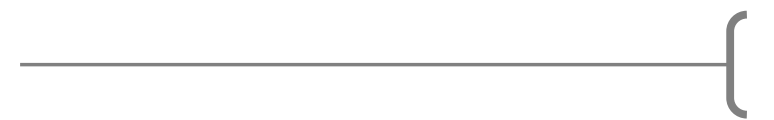

will be used in the final report. Upon completion of the research, the information will be published in a national and or international peer reviewed journal. The results will also be presented in national or international conferences. No names of the participants will appear on the publications.

Please find attached is the full proposal submitted to and approved by the Stellenbosch University Ethics Committee, a letter of approval from this committee, a shorten version of the proposal and data collection tools for your perusal.

I hope my request will reach your favourable consideration.

Yours Faithfully

Ntsikelelo Pefile (Mr)

\section{Pefilen@ukzn.ac.za}

\section{Gubela Mji}

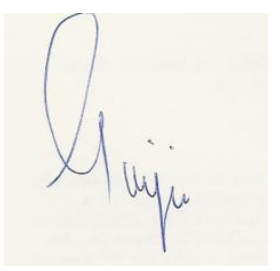

Ms S Gcaza

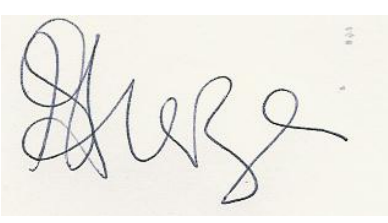

Head of Department Senior Lecturer (Supervisor) 


\subsection{Appendix G: Introduction To The Uk FIM+FAM}

\section{The UK FIM+FAM \\ (Functional Assessment Measure)}

Developed by the

UK FIM+FAM Users Group

Version 2.1

Brief overview

The full scoring manual, software and details of training courses may be obtained by contacting the UKROC team at the address below

For further information about the FIM+FAM please contact:

Professor Lynne Turner-Stokes DM FRCP

Regional Rehabilitation Unit

Northwick Park Hospital

Watford Road

Harrow, Middlesex, UK

HA1 3UJ

Tel: +44 (0) 20-8869-5161

Email: lynne.turner-stokes@dial.pipex.com

Secretary: jo.clark2@nwlh.nhs.uk

(The UK+FAM is adapted from the US version of the FAM, originally developed by Dr Karyl Hall and colleagues, Santa Clara Valley Medical Center, San Jose California, 1994) 


\section{Introduction}

The Functional Independence Measure (FIM) is an 18-item global measure of disability. Each item is scored on 7 ordinal levels. The FIM can be used for measuring disability in a wide range of conditions.

The Functional Assessment Measure does not stand alone but adds 12 FAM items to the FIM, specifically addressing cognitive and psychosocial function, which are often the major limiting factors for outcome in brain injury. Hence the Functional Assessment Measure is abbreviated to (FIM+FAM)

FAM items are rated on the same 7-level scale as the FIM items although the scaling structure of the FIM does not always lend itself to the more abstract nature of the FAM items.

The original FAM items were developed by Santa Clara Valley Medical Center (SCVMC), San Jose, California. However there were a number of problems for extrapolation to the UK settings:

1. Many items were written in US terms not easily transferable to UK settings

2. Some items were found to be too vague and subjective to score in routine practical use

In 1996 the UK FIM+FAM users group set about adapting the FAM items to produce a UK version. The UK FIM+FAM keeps the 7-level structure, but was adjusted to improve the objectivity of scoring, especially for the more subjective items. This work has been undertaken in collaboration with SCVMC.

The FIM+FAM is designed for measuring disability in the brain-injured population. The FIM data can be extracted and used on its own, for example when making comparison with populations in which only the FIM is rated.

The UKFIM+FAM also includes a 6-item module of extended activities of daily living, intended primarily for use in the community

For this reason it is important to score the FIM items as for the stand-alone scale, and the FAM items as an add-on. Certain items contain overlapping information.

For example:

"Eating" is a FIM item and includes swallowing, while "swallowing" alone is a FAM item. Eating should still be rated on the basis that it includes swallowing, so that the integrity of the FIM score is maintained.

Similarly, "Expression" is a FIM item, and includes speech intelligibility (articulation, voice modulation etc.), while "Speech intelligibility" is also rated alone as a FAM item 
Update of FIM manual

The original UK FIM+FAM (Version 1) used version 4 of the FIM manual.

In the updated edition (UK FIM+FAM version 2), the FIM items have been adjusted to align them with the FIM version 5 , which is the version currently in use by the Australasian Rehabilitation Outcomes Centre (AROC).

The AROC training manual provides a more systematised approach to scoring, breaking down items into component tasks to determine the \% of task completed by the patient. 


\section{Basic scoring principles}

1. Function is assessed by clinicians on the basis of direct observation. This requires the raters to be familiar with the patient and the standards for rating are:

Admission: Within 10 working days of admission date (may be adjusted for short programmes or community)

Discharge: During the last week before discharge

2. Scoring is undertaken by a multi-disciplinary team. There are several different models for team scoring. When the team is new to the scale, it is often most instructive to score from scratch as a team, one team member acting as facilitator to read out the questions in the decision tree until an agreed score is reached. As users become more familiar, time maybe saved by dividing items among the team, and each team member rating their items prior to meeting for discussion.

If there is disagreement amongst the team when scoring any item -the lower score is taken.

3. The FIM+FAM is essentially a rating of the need for assistance.

The person is scored on what they actually do, on a day-to-day basis, not what they could do or might be able to do, in different circumstances.

The FIM rating is therefore dependent on the environment, which may or may not be disability-friendly

Note: The wording convention in decision trees

"Does the patient need (or require) help..." should be interpreted as:

"Does the patient have help"

4. Do not:

- Leave any score blank or enter N/A -score 1 if unable to assess

- Score in half points -use the lower score

5. The person scores 1 if:

- They do not perform the activity at all

- If help from 2 people is needed

- If they would be put at risk of injury if tested

- If the information is simply not available

(Note - the US system allows a score ' 0 ' for some FIM items on admission only. This is because of their tight timescale for assessment (within 48 hours of admission)

The Australian and UK systems do not record any ' 0 ' scores - except the UK allows a ' 0 ' for wheelchair locomotion only if a wheelchair is never used at all and so not applicable. This does not impact on the total scores as Wheelchair Mobility is an alternate item to walking. Anyone wishing to understand how this manual compares with the US system may contact the Regional Rehabilitation Unit at Northwick Park Hospital - we can provide a manual which explains the differences. ) 
6. Two instructions differ from the original FIM manual for the UK FIM+FAM:

- For locomotion: record for both wheelchair (if applicable) and walking at each time point. Record the preferred mode on the score sheet.

- For bladder and bowel management -record both the level of assistance and the frequency of incontinence

These are recorded to support automated conversion to a Barthel Index

7. Use the decision trees and then check the level description with the notes at the bottom of the page to ensure the score is correct.

8. If function is variable for a given item, score the lower. 


\section{Description of the levels or function}

INDEPENDENT

7

6

DEPENDENT
Another person is not required for the activity (No helper) Complete independence The person performs all of the tasks described as making up the activity within a reasonable amount of time, and does so safely without the need for modification, assistive devices or aids. (No help, no devices, safe and timely!) Modified independence One or more of the following may be true:

a) requires an assistive device

b) takes longer than the reasonable amount of time

c) there is some concern for safety

(No help, but uses a device, or issues for safety or timeliness)

Requires help from another person to perform the activity, or the activity is not performed (Requires helper)

(Rating is based on observation. Therefore 'Requires help' means ' $\underline{\mathrm{Has}}$ $\underline{\text { help' }}$

Modified Dependence: The person performs more than half the task themselves

Requires no more than stand-by cueing, coaxing or verbal prompting without physical contact, or help just needed to set-up equipment, apply orthosis, etc.

(No help, but set-up or verbal prompting)

4

Minimal assistance

No more help than touching

Requires incidental help only to complete the task -does $75 \%$ themselves

(Help at the level of touching only - Madonna item!)

3

Moderate assistance

More help than touching

Requires moderate help, but still performs $50-74 \%$ of the task themselves

(Hands-on help but patient does more than half the task themselves)

Complete Dependence: The person performs less than half the task

Maximal assistance

Requires substantial assistance -the person provides $25-49 \%$ of the effort to complete the task

(Patient does less than half the task themselves, but does contribute) 
Total assistance

Requires total assistance -the person contributes less than $25 \%$ of the effort or the activity is not performed

Patient unable or does very little $(<25 \%)$ of the task 